\title{
The Home Treatment as a Supporting Method of Bobath for Treatment of Children with Cerebral Palsy in Kuwait
}

\author{
AlMattar, Mona
}

How to cite:

AlMattar, Mona (2019) The Home Treatment as a Supporting Method of Bobath for Treatment of Children with Cerebral Palsy in Kuwait. Doctoral thesis, Swansea University.

http://cronfa.swan.ac.uk/Record/cronfa50199

Use policy:

This item is brought to you by Swansea University. Any person downloading material is agreeing to abide by the terms of the repository licence: copies of full text items may be used or reproduced in any format or medium, without prior permission for personal research or study, educational or non-commercial purposes only. The copyright for any work remains with the original author unless otherwise specified. The full-text must not be sold in any format or medium without the formal permission of the copyright holder. Permission for multiple reproductions should be obtained from the original author.

Authors are personally responsible for adhering to copyright and publisher restrictions when uploading content to the repository.

Please link to the metadata record in the Swansea University repository, Cronfa (link given in the citation reference above.)

http://www.swansea.ac.uk/library/researchsupport/ris-support/ 
The Home Treatment as a Supporting Method of Bobath for Treatment of Children with Cerebral Palsy in Kuwait

By

Mona Al-Mater

Submitted to Swansea University in fulfilment of the requirements for the Degree of Doctor of Philosophy

School of Medicine

Swansea University

2019 


\begin{abstract}
Physiotherapy is one of the main management interventions for children with cerebral palsy. The Bobath approach is considered to be the most popular approach of physiotherapy for children with cerebral palsy even though it may not be the most beneficial approach if applied solely. To provide a holistic effective approach of physiotherapy to children with cerebral palsy the families are expected to apply specific handling and management techniques at home to support clinical physiotherapy management. In Kuwait outcomes of clinical management show deterioration with time and lack of home treatment application is suspected to be the main cause of decreased maintenance of achieved clinical physiotherapy functional outcomes. Therefore, the aim of this research was to identify the causes of such deteriorations of outcomes and a method of promoting home treatment adherence by families emphasize the importance of applying the home treatment in Kuwait in addition to identifying the most suitable follow up method to promote adherence to the home management. To achieve this a systematic review was performed to identify the effects of home treatment in supporting the clinical physiotherapy management and the most effective method to promote the adherence of families to the application of home treatment to be applied in Kuwait. Results have shown the home treatment to be a significant method of supporting clinical management and a variety of follow up methods were found to encourage families to apply home treatment. The documentation of physiotherapy procedures in the paediatric department in PMR hospital in Kuwait was then evaluated via an audit retrospectively and limitations in the educational process as well as the validity of goal setting was identified. To overcome these aspects of malpractice a pilot RCT showed the impact of implementing an educational course prior to physiotherapy interventions has had positive results in functional level in children with cerebral palsy in Kuwait especially when an objective outcome measure was applied. Families that received only educational lectures were found to be more adherent to the application of home treatment and their children have scored higher levels of change in functional abilities in the gross motor functional measure, compared to the families that received the educational lecture in addition to a weekly home visit as a follow up method. Families showed a contradictory response to the follow up method in the qualitative questionnaire to evaluate the level of parents' satisfaction, however, all families participating in the pilot study in Kuwait have reported benefits from the educational lecture. The physiotherapists in Kuwait agreed that the family education and home treatment plays an important role in supporting clinical outcomes when opinions were evaluated via an electronic survey. It was concluded that increasing awareness of cerebral palsy outcomes and the role of physiotherapy in the management of the disorder was found to be the main influence in improving home treatment adherence and outcomes, however home visits as a follow-up method did not show the expected effects in improving adherence. The educational lectures were also found to achieve high levels of family satisfaction and positive feedback regarding being part of this study in addition to being agreed upon as an effective method of support by physiotherapists in Kuwait. Overall, the data suggests that physiotherapy, when combined with additional treatment approaches, can be highly beneficial towards children with cerebral palsy, as well as their family.
\end{abstract}




\section{Acknowledgement}

At the end of this journey I would simply like to express how much this experience had influenced my personal and professional life. It had made me more patient, determined, and definitely stronger than I have ever been. It has been a long and difficult experience of learning new skills in the research field.

It is in this moment that I would like to thank God for all his blessings that he had provided me in life including this achievement. I would furthermore like to thank my loving husband who had supported me throughout this journey and whom after God I would have never made it so far without his support. 


\section{Table of Contents}

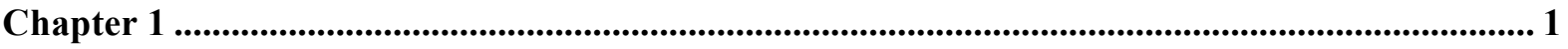

Introduction ................................................................................................................................................. 1

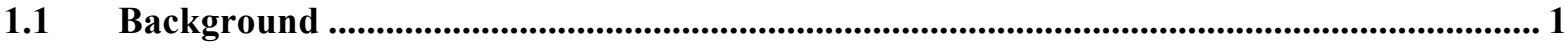

1.2 Definition of Cerebral Palsy ............................................................................................................ 2

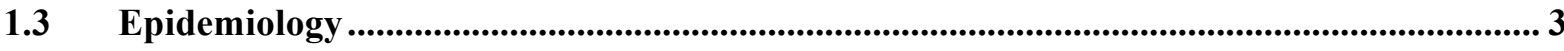

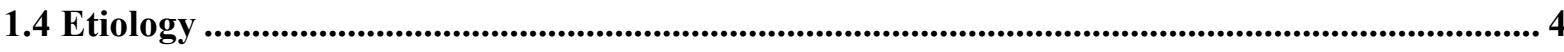

1.5 Diagnosis of Cerebral Palsy .......................................................................................................... 8

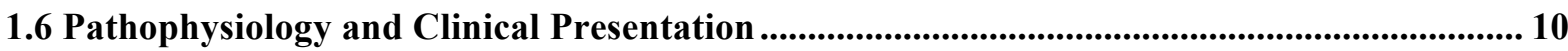

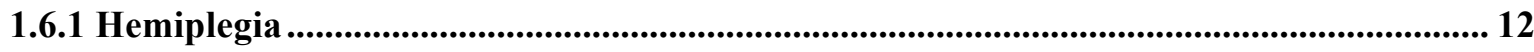

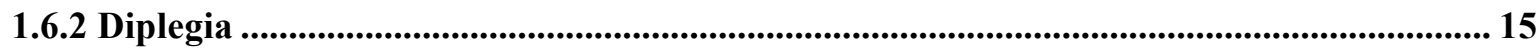

1.6.3 Quadriplegia ............................................................................................................................ 17

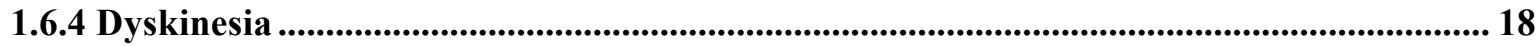

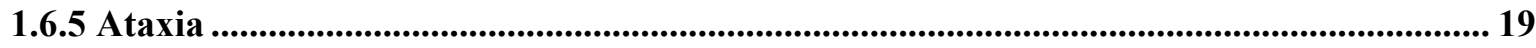

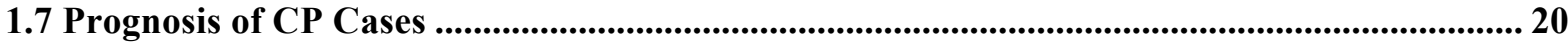

1.8 Management of CP ....................................................................................................................... 21

1.8.1 Physiotherapy Management .......................................................................................................... 21

1.8.1.1 Gross Motor Function Classification System .......................................................................... 27

1.8.1.2 Gross Motor Functional Measurement................................................................................... 28

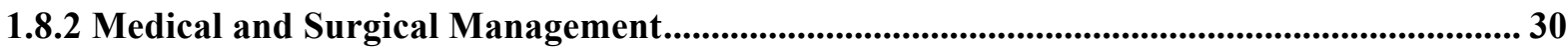

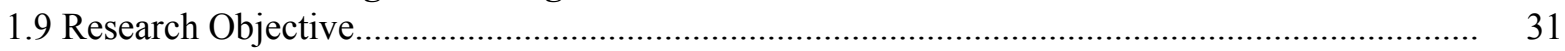

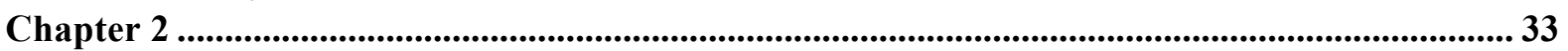

Physiotherapy Treatment for Children with Cerebral Palsy: A Narrative Review ....................... 33

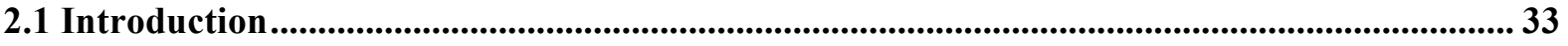

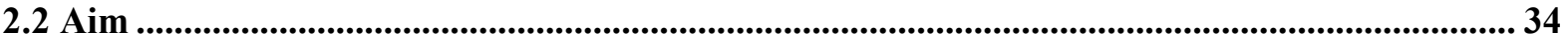

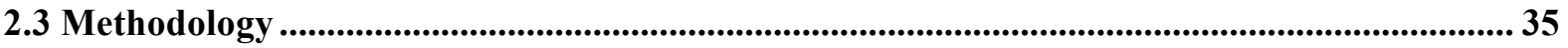

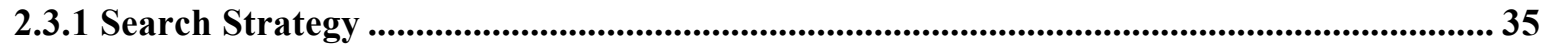

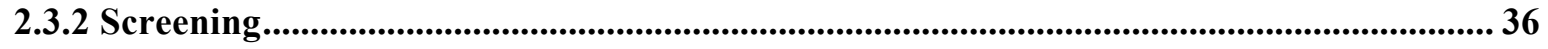

2.3.3 Eligibility and Inclusion Criteria........................................................................................ 36

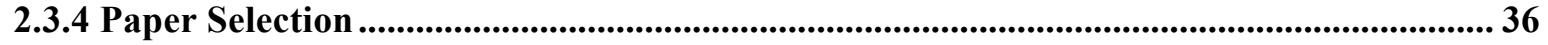

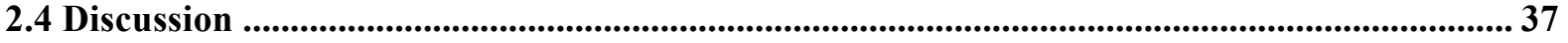

2.4.1 Child Focused Therapy and Context Focused Therapy......................................................... 37

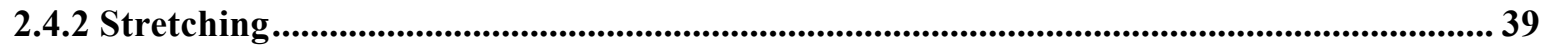

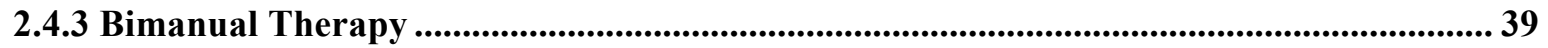

2.4.4 Constraint-Induced Movement Therapy .................................................................................... 40

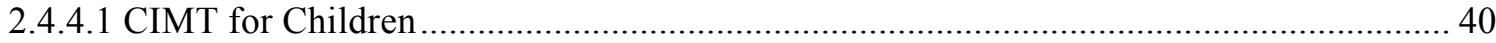

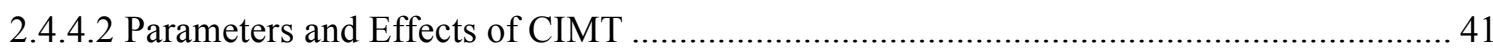

2.4.5 Strengthening .................................................................................................................................... 43

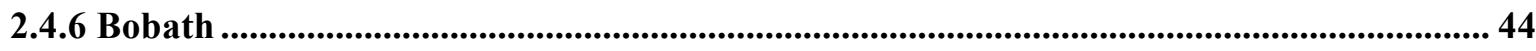

2.4.6.1 The effect of the Bobath Approach on Functional Level of Children CP ........................ 45

2.4.6.2 Comparison between the Bobath Approach and Other Interventions .............................. 49

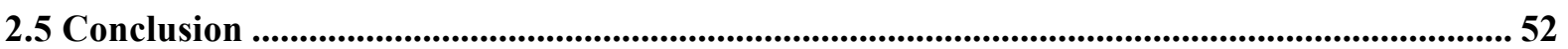


Chapter 3

The Effect of Home Treatment in Supporting Functional Development: A Systematic Review of the Literature ............................................................................................................................................. 54

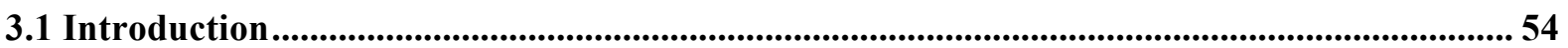

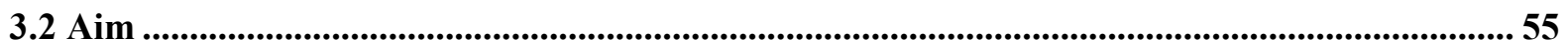

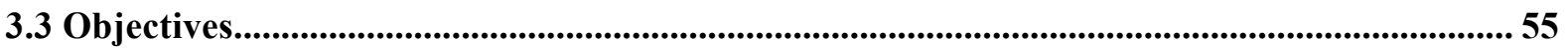

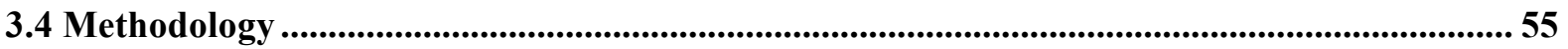

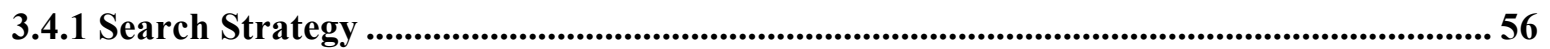

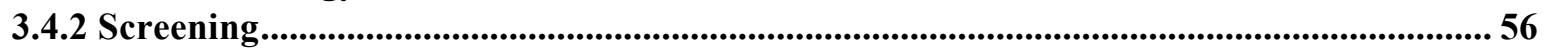

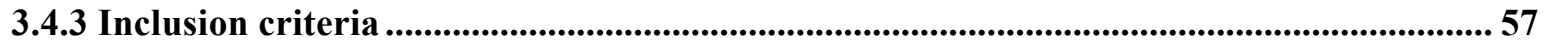

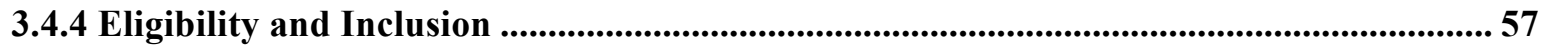

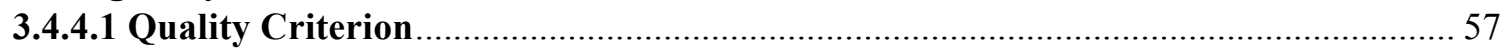

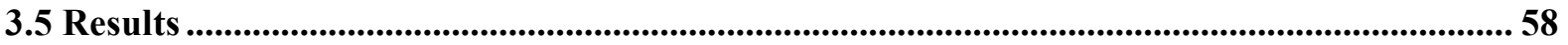

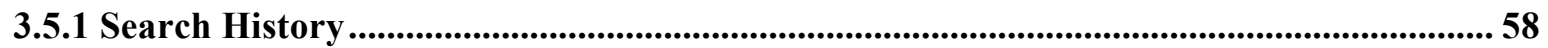

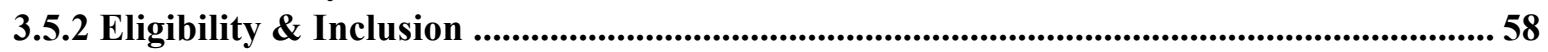

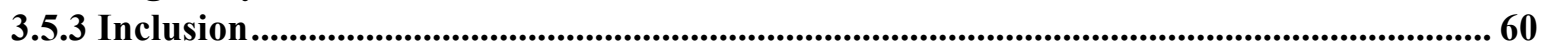

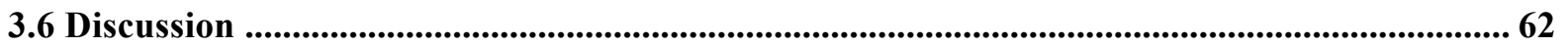

3.6.1 The Effect of Home Treatment on Functional Development..............................................62 62

3.6.2 Types and Methods of Home Treatment ..........................................................................68

3.6.3 The Level of Follow Up and Adherence ......................................................................................... 73

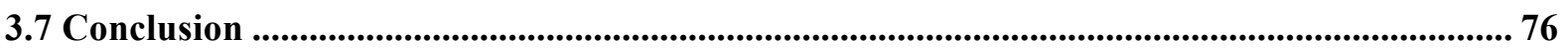

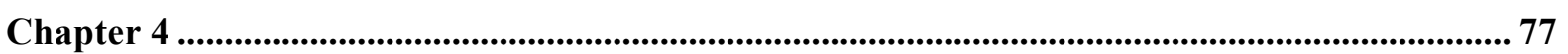

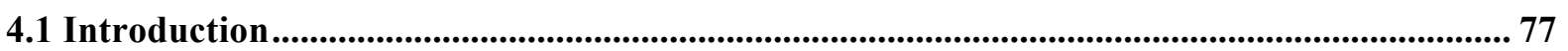

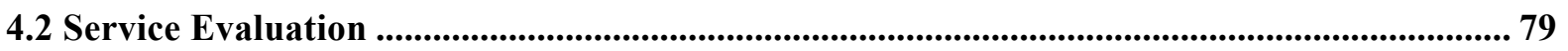

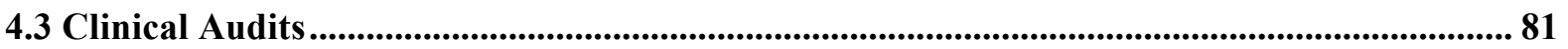

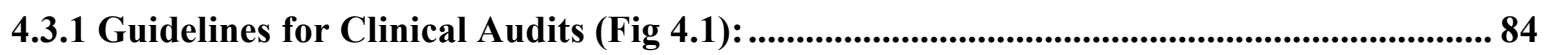

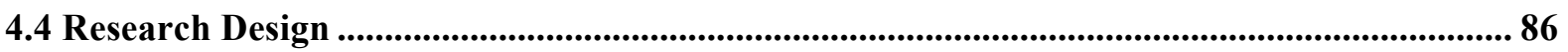

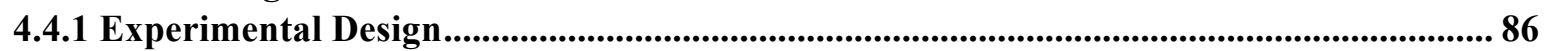

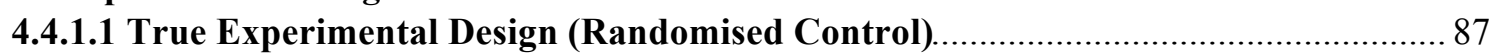

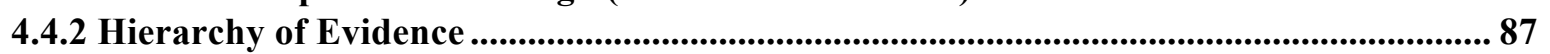

4.5 Non-Experimental Designs..................................................................................................................... 88

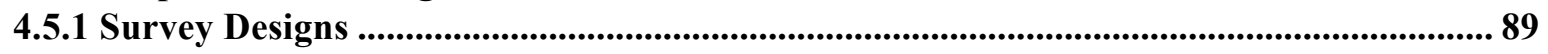

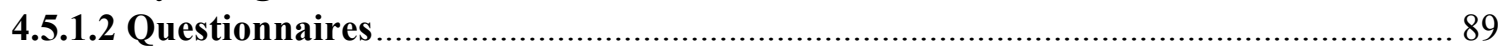

4.6 Methods of Project Studies ..................................................................................................... 92 4.6.1 Study 1 - A Clinical Audit: Evaluating Clinical Practice in the Pediatric Department of Physiotherapy in PMR Hospital in Kuwait ..........................................................................92 4.6.2. Study 2 - A Clinical Trial: Does Providing Parents Additional support for the delivery of a Home Program Improve the Functional Outcomes of Bobath? A pilot trial Study ......... 94

4.6.3 Study 3 - A Survey Measuring the Level of Satisfaction of Parents with the Pilot

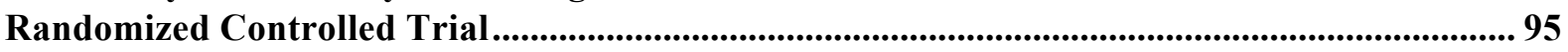

4.6.4. Study 4 - A Survey to Evaluate the Views of Physiotherapists on Bobath for Children

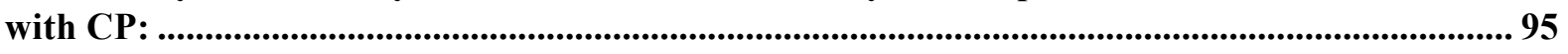

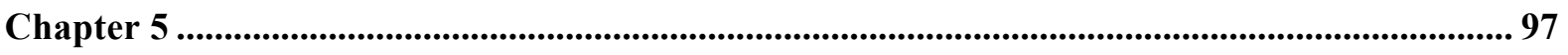


Evaluating Clinical Practice in the Pediatric Department of Physiotherapy in Physical Medicine and Rehabilitation Hospital in Kuwait: A Clinical Audit ...................................................................... 97

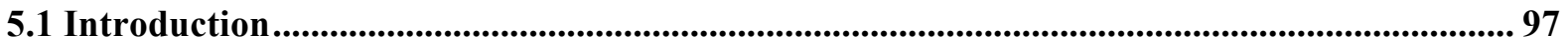

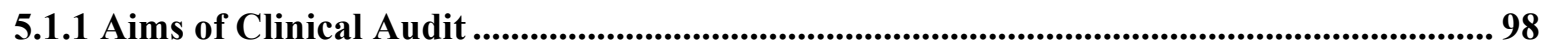

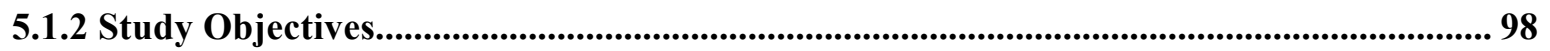

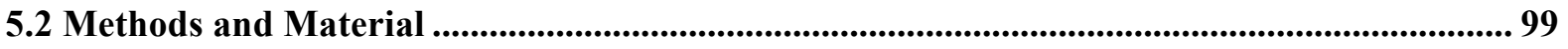

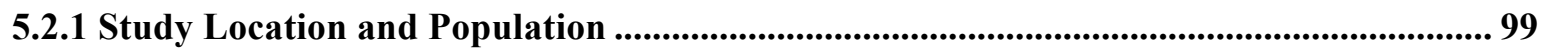

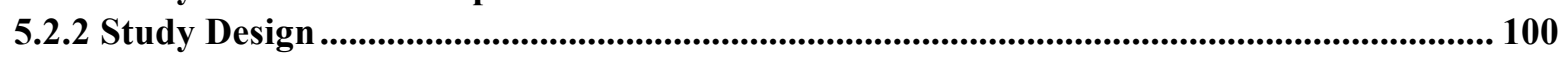

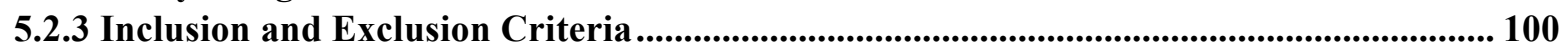

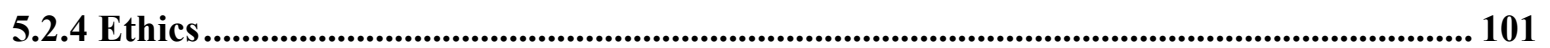

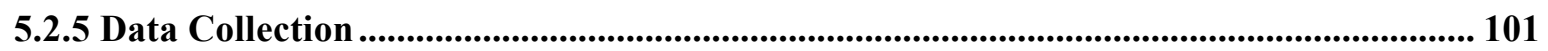

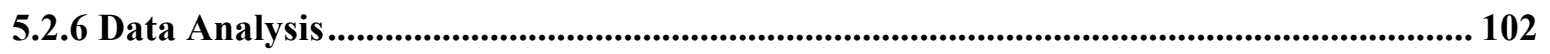

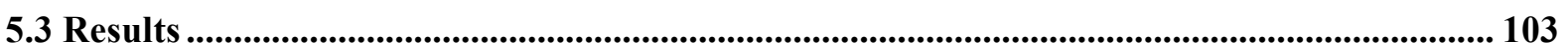

5.3.1 Information Regarding the Referral of the Patients .................................................... 104

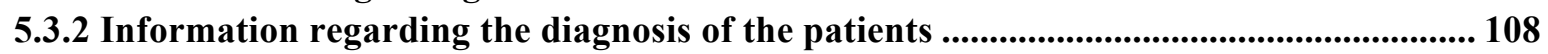

5.3.3 Information regarding the parameters of the treatment sessions .................................. 108

5.3.4 Information that includes documentation procedures ................................................... 109

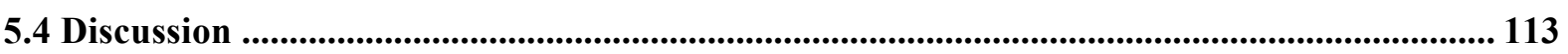

5.4.1 The Nature and Intensity of the Provided Physiotherapy Course for Children with CP

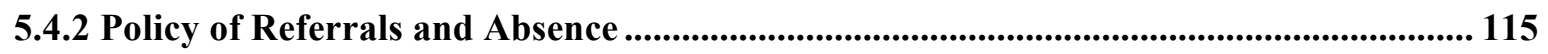

5.4.3 Cases Receiving Physiotherapy Services in the Department .............................................. 116

5.4.4 Physiotherapy Treatment Intensities ...................................................................... 116

5.4.5 Validity and Reliability of Outcome Measures ........................................................................... 118

5.5 Conclusion ............................................................................................................................................ 122

5.5.1 Recommendations for Service development................................................................................. 122

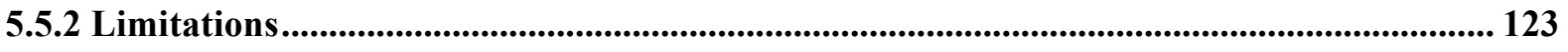

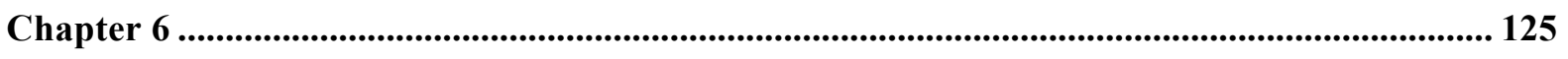

Does Providing Parents Additional Support for The Delivery of a Home Program Improve the

Functional Outcomes of Bobath? A Pilot Trial Study........................................................................... 125

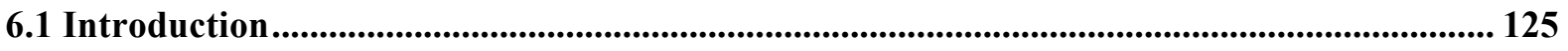

6.2 Aim of pilot study ................................................................................................................................. 126

6.3 Study Objectives....................................................................................................................................... 126

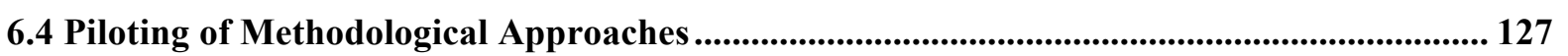

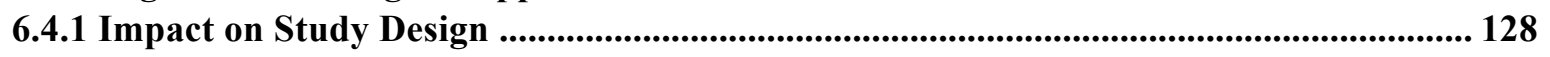

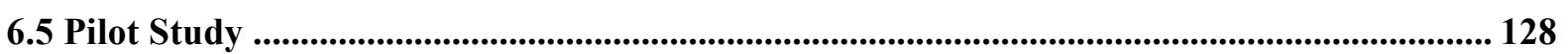

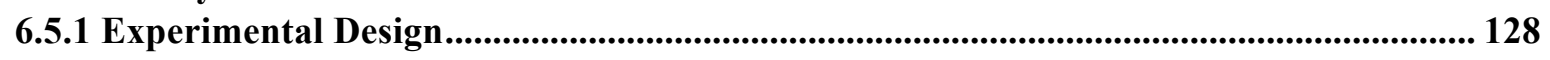

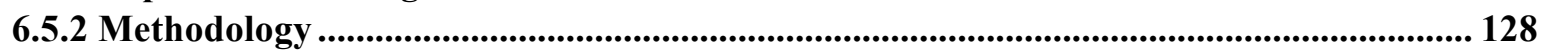

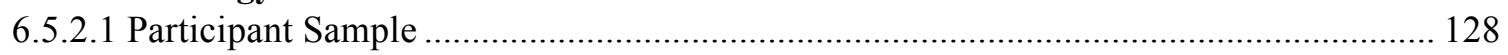

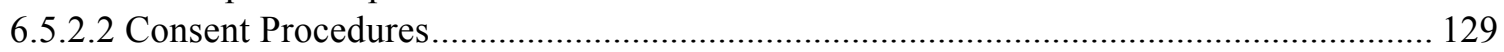

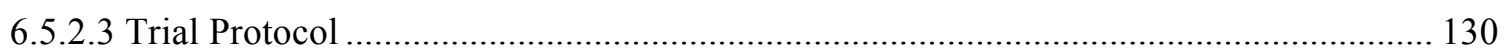

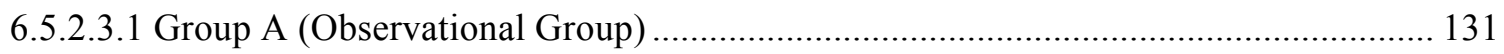

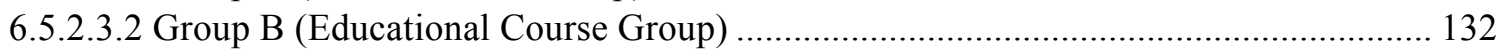

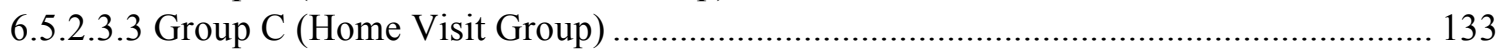

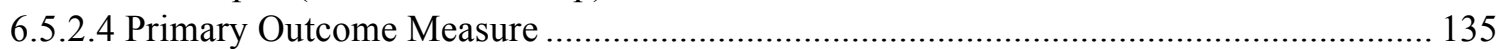




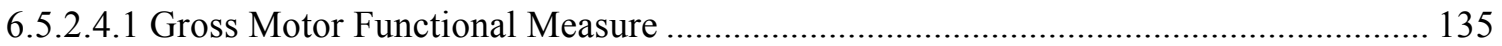

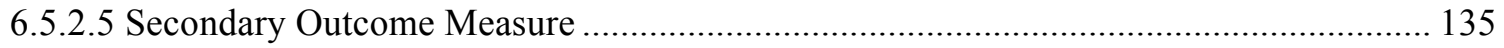

6.5.2.5.1 Parents Evaluation of Previous Service Questionnaire............................................... 135

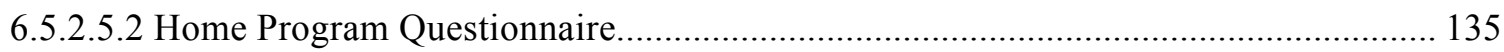

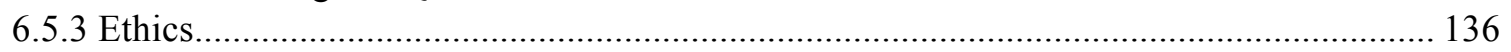

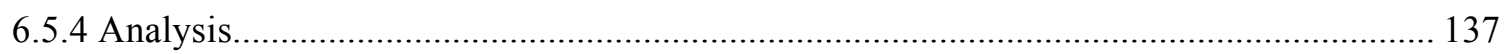

6.5.4.1 Previous Service Experience Questionnaire and Home Program Questionnaire ........... 137

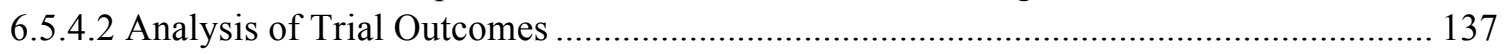

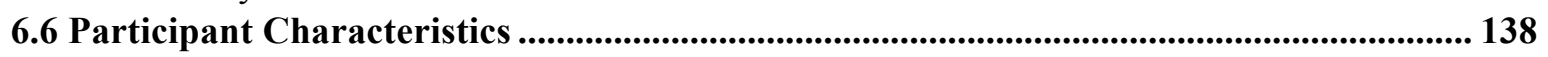

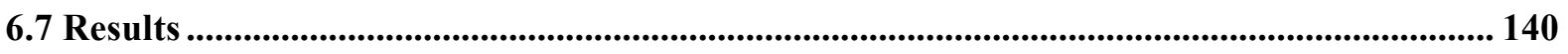

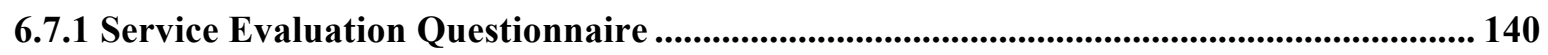

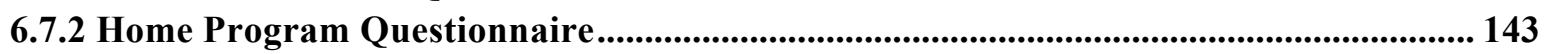

6.7.3 Assessing the Impact of the Parameters of the Trial on the GMFM Scores ................... 148

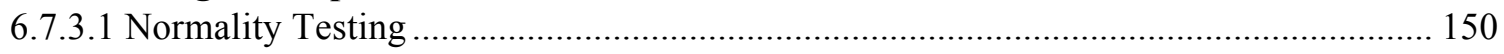

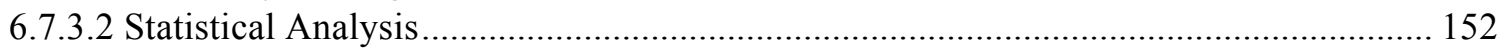

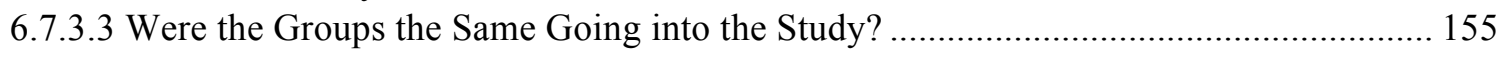

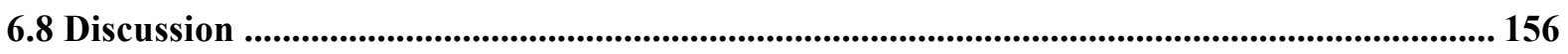

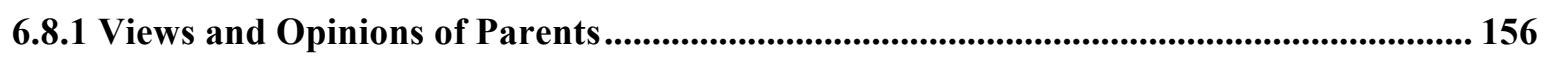

6.8.2 Effectiveness of the Different Parameters of Support to Promote Adherence................ 158

6.8.3 Feasibility of the Trial ....................................................................................................................... 160

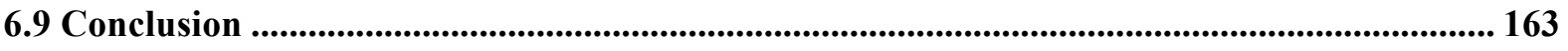

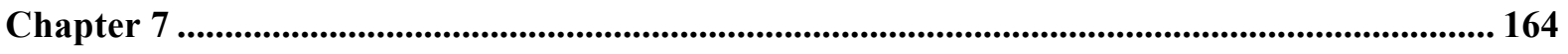

Measuring the Level of Satisfaction of Parents with the Pilot Randomized Controlled Trial: A

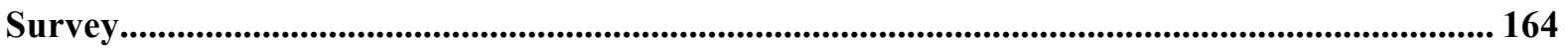

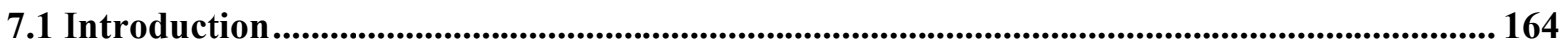

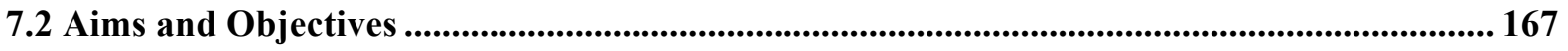

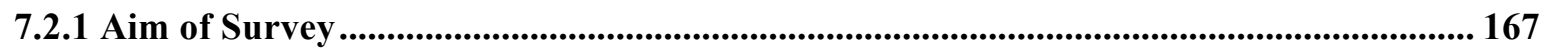

7.2.2 Study Objectives............................................................................................................................... 167

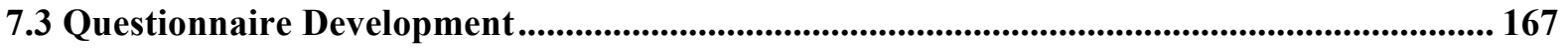

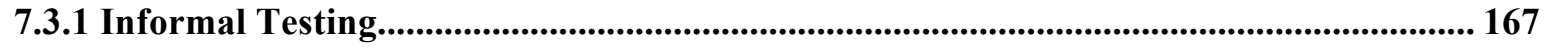

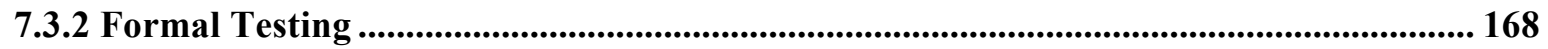

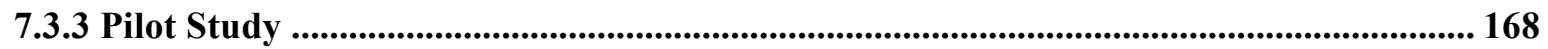

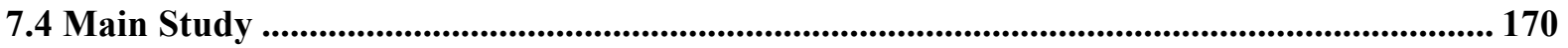

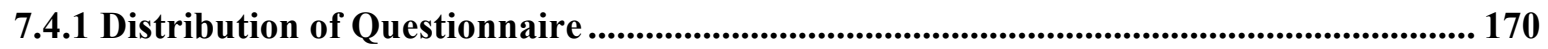

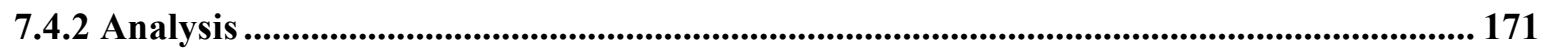

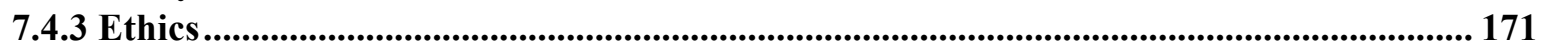

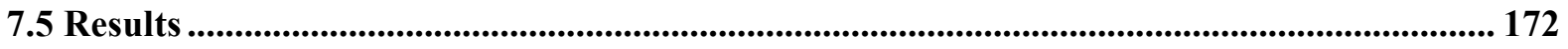

7.5.1 Comparison of Views Between Groups................................................................................ 180

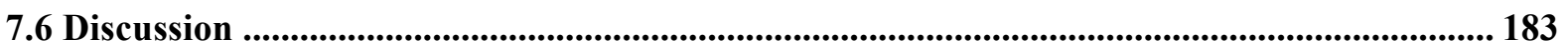

7.6.1 Level of Successful Communication Between the Parents and Researcher .................... 184

7.6.2 Levels of satisfaction with the procedures of the Pilot Study ......................................... 185

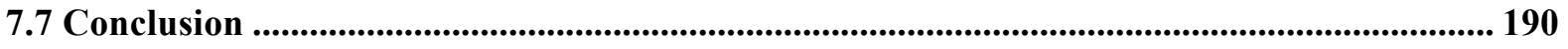

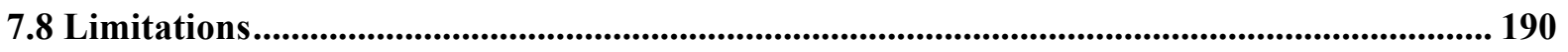

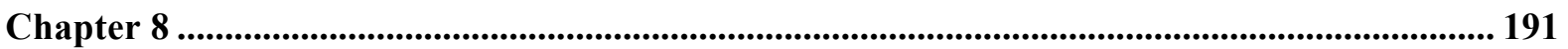


The Evaluation of the Views of Physiotherapists on Bobath for Children with CP in Kuwait: A

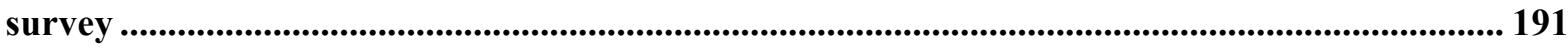

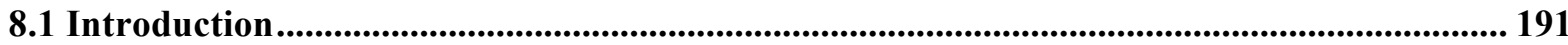

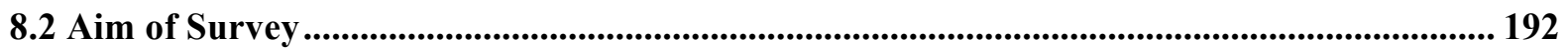

8.3 Study Objectives........................................................................................................................... 192

8.4 Development of Therapists Survey Questionnaire ......................................................................... 192

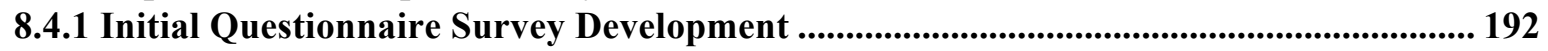

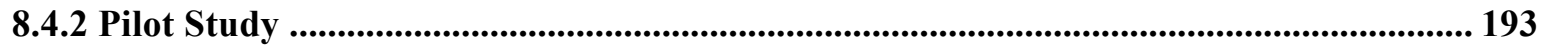

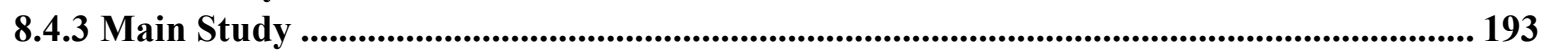

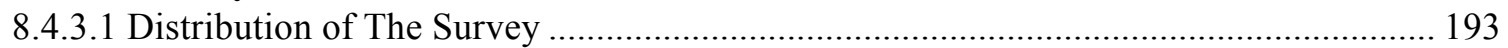

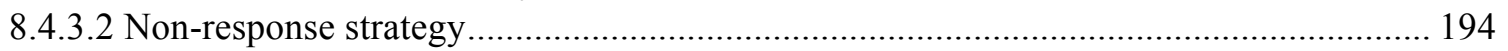

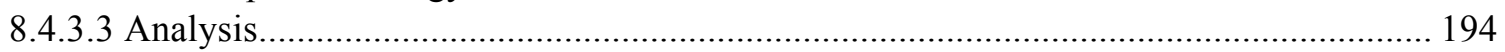

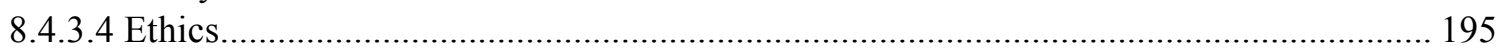

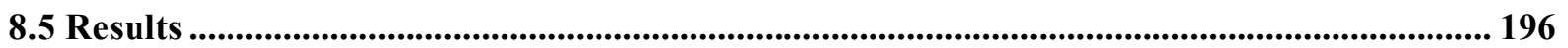

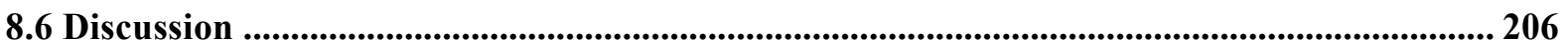

8.6.1 Information of the Personnel of the PMR Pediatric Department ................................... 206

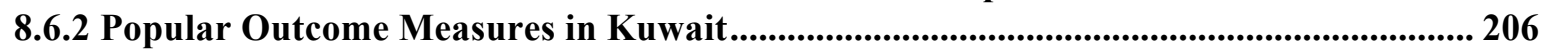

8.6.3 Views of Bobath Treatment ............................................................................................. 208

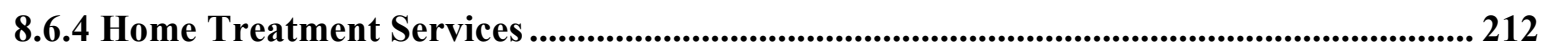

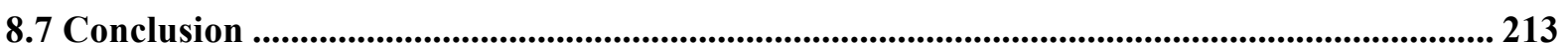

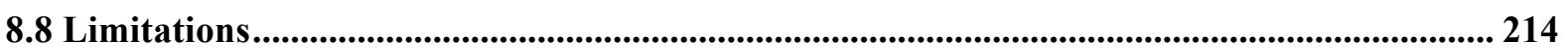

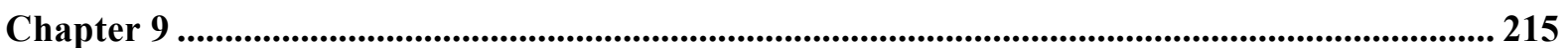

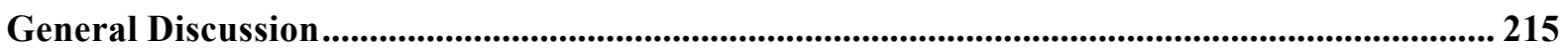

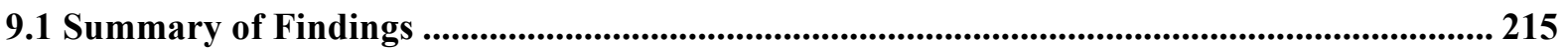

9.2 The Effects of the Main Physiotherapy Interventions in the Literature Identifying the Most Popular and most recent Physiotherapy Intervention Versus the Bobath Approach as the Most Common Type of Intervention in Paediatric Physiotherapy Management.

9.3 Identifying the Effects of Home Program in Supporting the Effectiveness of Clinical Physiotherapy Interventions Including Bobath Therapy Through a systematic review

9.4 Identifying Areas for Improvement in the Improper or Ineffective Delivered Services of Clinical Physiotherapy in the Physiotherapy Department in PMR Hospital to be Compared with the Results Learned from the Literature to be Reimplemented in Future Practice. .................. 218

9.5 Evaluating the Outcomes of Improved Aspects of Physiotherapy Management by Involving the Families of Children with CP in a Pilot Clinical Trial that Includes Limitations Identified by the Clinical Audit to Improve the Outcomes of Physiotherapy Management in PMR Hospital.

9.6 Evaluating Qualitative Satisfaction Responses of Parents Regarding the Integrity and Validity of the Pilot RCT.

9.7 Investigation of Physiotherapists Opinions of Clinical Physiotherapy Management Featuring Bobath Therapy in Kuwait compared to the Best Practice that they Believe in addition to Identifying the Factors Affecting these Opinions........................................................... 222

9.8 Recommendation for Research....................................................................................................... 224

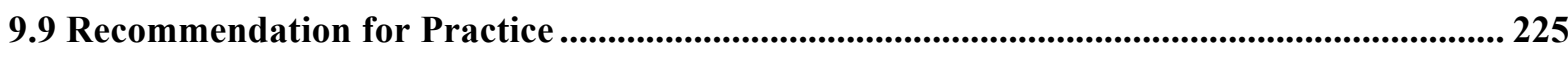


9.10 Impact on Practice .......................................................................................................................... 225

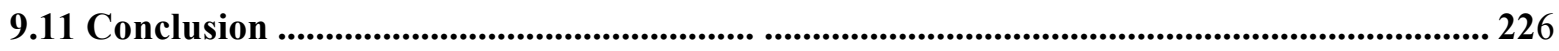

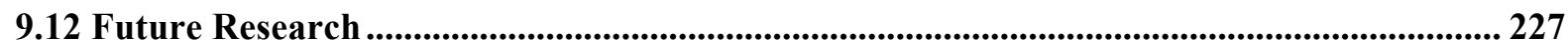

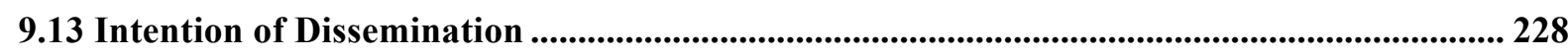

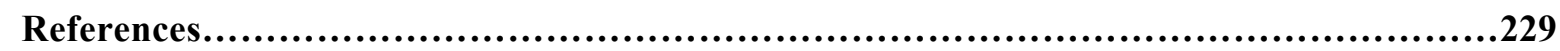

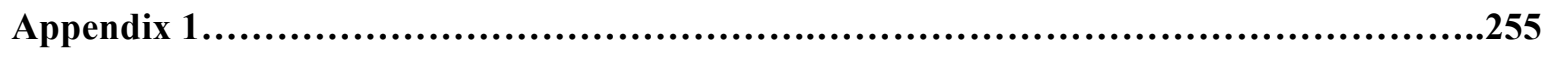

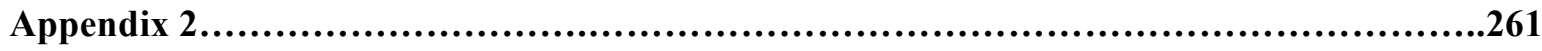

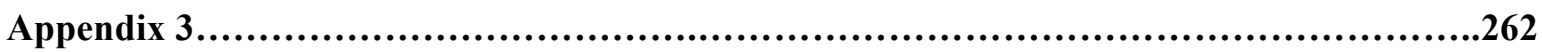

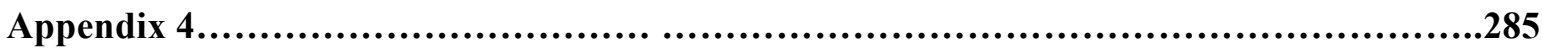

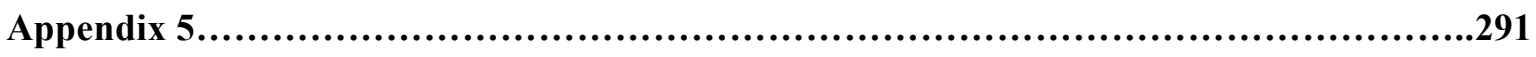

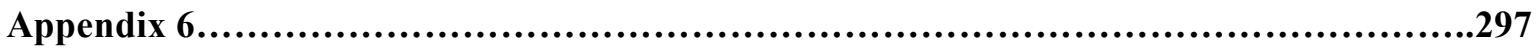

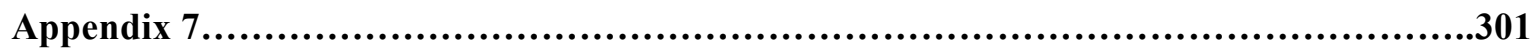

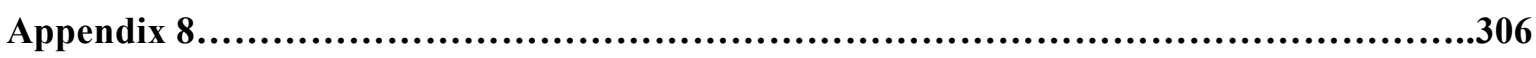

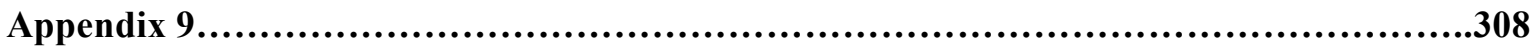

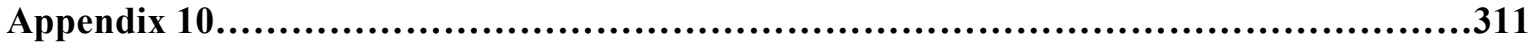

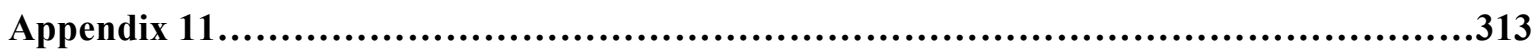

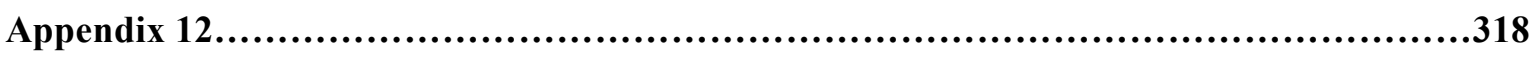

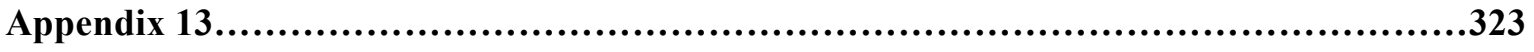




\section{List of Abbreviations}

$\begin{array}{ll}\text { CP } & \text { Cerebral Palsy } \\ \text { MRI } & \text { Magnetic Resonance Imaging } \\ \text { MRS } & \text { Magnetic Resonance Spectroscopy } \\ \text { UK } & \text { United Kingdom } \\ \text { VLBW } & \text { Very Low Birth Weight } \\ \text { ICU } & \text { Intensive Care Unit } \\ \text { \% } & \text { Percent } \\ \text { LBW } & \text { Low Birth Weight } \\ \text { GMH } & \text { Germinal Matrix Haemorrhage } \\ \text { PVH } & \text { Periventricular Haemorrhage } \\ \text { IVH } & \text { Intraventricular Haemorrhage } \\ \text { PVL } & \text { Periventricular Leukomalacia } \\ \text { AIDS } & \text { Auto Immune Deficiency Syndrome } \\ \text { CT } & \text { Computed Tomography } \\ \text { ITB } & \text { Intrathecal Baclofen } \\ \text { Botox } & \text { Botulinum Toxin } \\ \text { GMFCS } & \text { Gross Motor Function Classification System } \\ \text { GMFM } & \text { Gross Motor Functional Measure } \\ \text { CIT } & \text { Constraint Induced Therapy } \\ \text { NDT } & \text { Neurodevelopmental Therapy } \\ \text { CINAHL } & \text { Cumulative Index to Nursing and Allied Health Literature } \\ \text { PEDI } & \text { The Pediatric Evaluation of Disability Inventory } \\ \text { MAS } & \text { Modified Ashworth Scale } \\ \text { H } & \text { Hoffman Reflex } \\ \text { Minutes }\end{array}$

AACPDM American Academy of Cerebral Palsy Developmental Medicine 


$\begin{array}{ll}\text { ROM } & \text { Range of Motion } \\ \mathrm{m} & \text { Meters } \\ \mathrm{CE} & \text { Conductive Education } \\ \text { ADL } & \text { Active Daily Living } \\ \text { CIMT } & \text { Constraint Induced Movement Therapy } \\ \text { QUEST } & \text { Quality of Upper Extremity Skills Test } \\ \text { HABIT } & \text { Hand-Arm Bimanual Intensive Therapy } \\ \text { mCIMT } & \text { Modified Constraint Induced Movement Therapy } \\ \text { AHA } & \text { Assisting Hand Assessment } \\ \text { BiT } & \text { Bimanual Training }\end{array}$




\section{Chapter 1}

\section{Introduction}

\subsection{Background}

From my own clinical experience as a physiotherapist in the Paediatric Department of the Physical Medicine and Rehabilitation Hospital in Kuwait for three years, it was observed that the children with cerebral palsy that were receiving treatment in the department had not been showing the expected level of functional development. Furthermore, it is not achieving all their set goals in terms of maintaining or improving their levels of physical function. In addition, the children have been showing clear deterioration and loss of achieved functional abilities after returning to the department for a new course of physiotherapy after being away from treatment for a period that might reach six weeks. Therefore, the causes of these results were part of the interest of the researcher to perform this research.

This research will explore the physiotherapy management of cerebral palsy $(\mathrm{CP})$ using the Bobath therapeutic approach, which has been reported as being a highly prevalent neurodeficit in children around the world (Damiano, 2009). It will highlight incidence and prevalence as well as detail our current understanding of the pathophysiology involved in the clinical presentation of CP. The various types of the deficit will be discussed as well as the different levels of severity per measurement and classification tools that are clinically relevant.

The research will discuss medical and other management of the deficit including the physiotherapy role in rehabilitating patients to reach their maximum motor ability. The literature will be reviewed for various types of physiotherapy approaches that would be discussed and evaluated including the Bobath approach. It is an approach that focuses on sensory-motor components of muscle tone, reflexes, and abnormal movement patterns, postural control, sensation, and memory. It provides handling techniques that controlled various sensory stimuli used to inhibit spasticity, abnormal reflexes, and abnormal movement patterns, which are also used to facilitate normal muscle tone. As additional knowledge of neuroscience became available, the approach had changed in certain aspects of the treatment (Butler \& Darrah, 2001). 
Therefore, this research will explore the effectiveness of involving the family in supporting the application of the Bobath approach for children with CP. Studies included within this research will include a clinical audit that will evaluate current provision of physiotherapy led CP services, to provide a description of current practice within Kuwait, with a focus on the delivery and treatment parameters of Bobath; a questionnaire that explores parents experience of physiotherapy management of their children with $\mathrm{CP}$ to determine levels of satisfaction; a trial that will evaluate the benefits (or not) of including a supportive home program in addition to the delivery of the current parameters of treatment (as identified by the audit); a survey exploring parents experience of receiving supportive home program and finally, a questionnaire survey that will explore physiotherapists opinions of CP management approaches and practice.

\subsection{Definition of Cerebral Palsy}

As this research aims to explore the potential effects of including a supportive home program as part of a Bobath approach in the management of $\mathrm{CP}$, it is important to provide an overview of $\mathrm{CP}$ in terms of prevalence, pathophysiology, clinical presentation, and potential management strategies.

Cerebral palsy refers to a non-progressive or episodic central nervous system deficit (Butler \& Darrah, 2001). Outcomes may become more obvious in early childhood, however, no qualitative changes are observed. It is a descriptive non-specific term that pertains to disordered motor function, which can be seen in early infancy and can be characterised by muscle tone, involuntary movement, ataxia or a combination between all of these abnormalities (Sankar \& Mundkur, 2005). The lesion can be found in single or multiple locations of the brain, therefore, it could be seen as motor abnormalities, in addition to sensory in some cases that are accompanied by other associated disabilities (Wood, 2006). Motor abnormalities usually affect the limbs as well as the trunk in some cases. Sensory and intellectual abnormalities may be seen in cerebral palsy cases, however, they are not included in the diagnostic criteria. The term cerebral palsy is still confusing and confounding for various reasons. It does not include the degree of involvement or severity of the diagnosis. It also does not include any other underlying condition that a child may have. Although these conditions may play a major role in affecting the child's motor abilities and impairments, still, the prevalence is modified in age group due to the prognosis that accompanies 
maturation. However, approaching cerebral palsy regardless of the other underlying conditions supports the delivery of management of the deficit, as all children commonly undergo similar medical, social, and rehabilitative care. Diagnosis of cerebral palsy is referred to different factors that will be further discussed.

\subsection{Epidemiology}

The rate of Cerebral palsy is measured per thousand live births and not per population alive at the time of diagnosis. This is called prevalence and not incidence (Scherzer, 2000). It is due to the nature of the deficit of CP where there is a considerable gap of time between the onset of CP and its diagnosis. Therefore, a number of cases die or are lost to follow up before the population is counted worldwide, which makes it difficult to calculate the correct incidence of cerebral palsy per thousand populations alive around the world (Scherzer, 2000). In industrial countries of the world, $\mathrm{CP}$ is counted in the range between 2 and 2.5/1000 live births (Himmelmann et al., 2007; Scherzer, 2000). It is believed to be higher in developing countries in comparison to industrial countries due to the higher numbers of asphyxia and low birth weight. However, there are no population studies to support the exact numbers of the prevalence of CP. It had been found that CP has no relation with ethnicity throughout the world but was believed to be more common in ethnic groups in whom consanguineous marriage is common such as Pakistani immigrants in the UK and Saudi Arabians (Scherzer, 2000).

Other studies have suggested that the number of males with $\mathrm{CP}$ is higher compared to females, especially in severe cases that have dysmorphic features (Scherzer, 2000). However, no explanation yet exists for these findings (Scherzer, 2000). Studies also suggest an increase in the numbers of CP cases in industrial countries in the 1980s compared to the 1970s in very low birth weight infants, despite the advances in obstetric and neonatal care (Reddihough \& Collins, 2003). This increase in prevalence seems to be due to the improved survival of Very Low Birth Weight (VLBW) infants in whom the prevalence of CP is very high (Miller, 2005). This can be contributed to the Intensive Care Unit (ICU).

Despite the stable incidence of disability among the VLBW their survival alone is likely to increase CP prevalence (Reddihough \& Collins, 2003). The prevalence of CP in 
infants below $1500 \mathrm{~g}$ is 60 times the prevalence of CP in infants with weights above $2500 \mathrm{~g}$. As the number of CP is increasing among the VLBW population, the clinical types of CP have also changed (Miller, 2005).

\subsection{Etiology}

Cerebral Palsy is considered multifactorial like many other diseases with conditions working in tandem or simultaneously to cause it (Scherzer, 2000). Therefore, there should be no rush to refer $\mathrm{CP}$ to a perinatal cause without understanding the prenatal events of each case (Aicardi, 1992). Eighty-five percent of CP cases considered congenital types that are caused by intrauterine natal and perinatal causes (Aicardi, 1992). Prematurity or low birth weight stands for more than $40 \%$ of cases of CP (Maclennan, 1999). It is 100 times more likely to diagnose $\mathrm{CP}$ in infants with low birth weight compared to normal weight infants. As low birth weight (LBW) is a major factor of $\mathrm{CP}$, it is still not clear exactly what is causing preterm and therefore LBW (Eriksson et al., 2006). Some pathogenetic mechanisms suggested prenatal antecedents causing early brain damage which led to early delivery and others suggested antecedents causing the early delivery which led to brain damage (Maclennan, 1999; Scherzer, 2000).

What is known is that neurodevelopmental sequelae is caused by damage to the white matter in the preterm infant's brain known as perinatal leukoencephalopathy (MacLennan, 1999). This condition includes various symptoms and conditions that include many types of haemorrhage that include Germinal Matrix Haemorrhage (GMH), Periventricular Haemorrhage (PVH), Intraventricular Haemorrhage (IVH) (Fig.1.2), periventricular haemorrhagic infarction and Periventricular Leukomalacia (PVL) (Fig.1.3) (MacLennan, 1999) (Fig 1.1). Infarctions usually occur due to the sensitivity of the infant's cerebral circulation to change in blood pressure especially that infants lack the glial support in their germinal matrix (MacLennan, 1999, Scherzer, 2000). This causes obstruction in the terminal veins and usually lead to haemorrhagic infarction (Scherzer, 2000). PVL, on the other hand, is due to ischemic necrosis in the border zone of long penetrating arteries of the periventricular white matter (Scherzer, 2000). 
In such injuries the damage may extend beyond the white matter area into the subcortex and beyond, resulting in loss of oligodendrocytes and increase in hypertrophic astrocytes affecting nerve cell growth and impairing myelination (Scherzer, 2000). LBW infants usually suffer conditions similar to respiratory distress syndrome, apnea, hypotension, infection and patent duct arteriosus which in turn result in hypoxia, acidosis and necrosis (Miller, 2005). Intra and periventricular haemorrhage are usually investigated by ultrasound and seen as a distortion of the contours of cerebral ventricles or ventriculomegaly cause a 6070 percent risk of CP (Miller, 2005). Echolucent areas, on the other hand, which are darker areas found with no echoes are very rare and are also detected by ultrasonography having a $100 \%$ risk of CP (Miller, 2005). Causes of premature deliveries are still unknown although they could be predicted in some cases related to the history of preterm delivery with the mother, smoking, placental infection and fetal malformation (Taylor et al., 2009).

Another major cause of $\mathrm{CP}$ is birth asphyxia. It occurs when the lungs or placenta fails at the time of birth causing oxygen deprivation, hypercarbia, and metabolic acidosis (Miller, 2005). Asphyxia would either be severe or total, which is uncommon and would involve the thalamus, brainstem and basal ganglia sparing the cerebral cortex or would be partial and prolonged, which is the most common (Scherzer, 2000). In fact, infants' asphyxia would lead to a bilateral parasagittal watershed injury, on the other hand, asphyxia in preterm infants would result in a bilateral periventricular end and border zone injury (Miller, 2005).

Neurodevelopmental damage, which usually exceeds the motor system to intellectual deficit and epilepsy usually occur as a result of an extreme and prolonged episode of asphyxia (Miller, 2005). Birth asphyxia usually ranges between 1-2 per 1000 full-term infant and up to $60 \%$ of preterm infants (Sankar \& Mundkur, 2005; Scherzer, 2000). Most full-term infants survive (60-80\%) compared to $10-60 \%$ of preterm infants with asphyxia expire (Sankar \& Mundkur, 2005). Birth Asphyxia is now an outdated term that falsely conveys that a newborn with signs of neonatal compromise must have had an acute hypoxic incident in late labour or birth (MacLennan et al., 2015). Although in the large majority of cases $(>76 \%)$, other causes such as infections or genetics are the causes of such neonatal compromise (MacLennan et al., 2015, Ahearne et al., 2016).

MacLennan et al. (2015) indicated that, as far as there is limited recent evidence that can be used to refer to the signs of encephalopathy as acute ischemia at birth, "Neonatal 
Encephalopathy" is the term that must replace "Hypoxic Ischemic Encephalopathy". This indicates the causes to be associated with either acute intrapartum timing or chronic longstanding timing of the pathologies, before labour and during pregnancy (MacLennan et al., 2015). Other factors such as infection, placental and umbilical vessel thrombosis have an effect in promoting neonatal encephalopathy. Such incidents that directly affect the brain leads the infant to being more vulnerable to intrapartum stress, which in turn may lead to $\mathrm{CP}$ (Miller, 2005). Recent scientific evidence refers CP to genetic mutation, and environmental triggers such as bacterial and intrauterine infection, intrauterine growth restriction, intrapartum haemorrhage, and threatened miscarriages rather than birth asphyxia (MacLennan et al., 2015). This justifies the cases of CP were found not to be preventable by intrapartum obstetric or neonatal interventions. Although rates of electronic monitoring of fetal distress and caesarian sections have risen the prevalence of CP had not decreased (Miller, 2005 \& MacLennan et al, 2015).

Hyperbilirubinemia was considered one of the major factors of kernicterus which is a mild form of athetosis (Scherzer, 2000). However, its effect has been greatly controlled by methods of early identification. Neonatal jaundice, on the other hand, may still require some careful attention. Infections that may lead to sepsis or meningitis in the perinatal period in addition to Herpes simplex encephalitis are significant in causing brain damage leading to $\mathrm{CP}$ as well as intellectual deficit (Scherzer, 2000).

CP can be caused by a number of prenatal factors that are responsible for more than $70 \%$ of the cases of CP (Miller, 2005), confirming the view that suggests that difficulty at birth is a result of the prenatal complications and not the other way around. In most of these cases, the factors cannot be prevented. In CP enamel hypoplasia is a common defect found in children suggesting a prenatal complication in the first trimester when enamels are formed (Panteliadis, 2011). Another popular prenatal factor that was seen in more than $30 \%$ of children with CP is a fetal deprivation of supply that is in itself a result of disorders such as bleeding during pregnancy, placental infarction, and twining (Panteliadis, 2011). Twins showed up to seven times the risk of CP when compared to a single pregnancy even when low birth weight and prematurity were controlled (Taylor et al, 2009, Panteliadis, 2011). Other prenatal factors are known to have an effect on the development of infants regardless of the gestational age or birth weight, however, no exact relationship to CP has been found yet. These are factors that include the general health status of the mother, radiation, drugs, 
smoking, and alcohol. Endocrine problems during pregnancy were found to have an association with a higher risk of CP (Miller, 2005; Panteliadis, 2011). Maternal infections such as congenital rubella, cytomegalovirus and herpes remain a major cause of central nervous system pathology, even though they might not show any noticeable clinical manifestation during gestation. Autoimmune Deficiency Syndrome (AIDS) is considered the latest congenital infection on the list that is related to severe developmental disorders and primary Central nervous system motor conditions (Miller, 2005; Panteliadis, 2011). Vascular factors could also be considered prenatal. Prenatal factors show the importance of not rushing to blame other factors present during delivery (Panteliadis, 2011).

In cases that have a higher incidence of $\mathrm{CP}$ in their offspring due to consanguineous marriage, there is evidence that cerebral palsy may be of a hereditary basis. There are some documentation of well-known cases that are genetically determined, which includes generalized athetosis, spastic paraplegia, and ataxia. Causes of such cases are seen as static encephalopathy due to vulnerability to metabolic toxins that are seen in Amish groups in Pennsylvania (Miller, 2005). More recent studies have considered possible genetic causes that may directly, or through genetic susceptibility, trigger different pathways to different neuropathologies that share the common clinical trait of CP. However, they are still inconclusive (MacLennan et al, 2015).

History of repeated miscarriages and abortions, long periods between pregnancies and even longer intervals between menstrual cycles are considered preconceptional factors that may cause CP (Taylor et al., 2009).

Since CP is considered as an injury to the developing brain, some studies have included brain damage sustained within the first month and up to 2 years of age as part of injury to the developing brain, given that the period in which the brain continues to develop is uncertain (Scherzer, 2000). Infections and traumatic injuries are the most common causes in that period. Infective cases including meningitis and encephalitis are the most common as well as herpes simplex infection in the developed countries (Scherzer, 2000). Trauma, on the other hand, is common in children once they start to walk and develop independence in preschool age, which includes motor accidents or near-drowning or choking as well as a shaken baby syndrome (Miller, 2005). Other cases are caused by neurodevelopmental sequelae due to untreated hydrocephalus, neoplastic intracranial lesions that occur in the early 
developing years (Miller, 2005). Postnatal cases of CP account for approximately 15\% of cases of $\mathrm{CP}$ which are considered totally preventable, unlike congenital cases of CP (Miller, 2005).

\subsection{Diagnosis of Cerebral Palsy}

Knowledge and understanding of the orderly sequence of development and integration behaviour is the key for identifying the limits in which deviation in maturation can occur. This becomes the first aspect in which abnormalities can be suspected and diagnosed. Infant neurological development is usually highly dependent on environmental influence; it has an exceedingly complex physiological basis. An insult to the central nervous system will be apparent generally as abnormalities in general movement, tone and developmental milestones rather than distinct and observable focal motor dysfunction (Himmelmann et al., 2007; Hall, 2009). This will lead to a challenge for those working to identify developmental problems in very young children and differentiate significant delays from limits of typical variability and whether it is normal or pathological. Predicting developmental abnormalities requires both physiological and developmental screening (Hall, 2009). There are always ways to correlate early distress symptoms with the prediction of development. In biochemistry, it started with the Apgar score in the 1950s which assessed the colour, respiratory effort, heart rate, tone, and reflex activity (Aicardi, 1992). However; poor correlations were found between the score and chronic neurological deficits later in life (Aicardi, 1992). Other assessments at the time similar to blood acidemia tests indicate that asphyxia was also poor indicators of neurological deficits when applied alone (Aicardi, 1992). Predictability was improved with a low five-minute score of Apgar score as well as the need of intubation at the birth time (Aicardi, 1992). Various spinal fluid tests may be considered good indicators of neurological deficits, however, they are not usually obtained without obvious evidence of neurological abnormality (Scherzer, 2000). Other tests would include urine assessments, which indicate damage to the kidneys due to asphyxia (Scherzer, 2000). Although biochemical tests indicate hypoxic ischemia, it also indicates injuries to the brain (Scherzer, 2000). Hypoxic injuries to the brain represent only one etiologic basis in many people for static encephalopathy (Himpens et al., 2010). Prenatal physiological screening also played an important role in indicating abnormalities. Tests similar to amniocentesis, chronic villus sampling as well as ultrasound, are essential tools for indicating 
developmental disabilities similar to Down syndrome (Wood, 2006). In premature and highrisk infants neuroimaging of ultrasonography, cranial tomography and Magnetic Resonance Imaging (MRI) have considerable capabilities for documenting peri and intraventricular lesions as well as demonstrating hypoxic-ischemic encephalopathy (Panteliadis, 2011). Moreover, most recently proton Magnetic Resonance Spectroscopy (MRS) is being used to observe abnormal intracellular metabolites within hours of birth, which indicates neurological deficits (Panteliadis, 2011). However, all these techniques still only deal with one possible aetiology of developmental disabilities (Panteliadis, 2011; Schrzer, 2000).

It has been recognised that early development is a dynamic and changing process, therefore, extensive efforts were made to anticipate and predict developmental abnormalities in very young children (Hall, 2009). A variety of screening instruments have been introduced in recent years with a variety of degrees of accuracy for prediction of developmental delay, definite developmental abnormalities, and specific developmental diagnosis. Such instruments vary in the degree of reliability, sensitivity, and validity and their predictive powers increase with the age of the child (Himpens et al., 2010). At present, there is no standardisation of these tests, and moreover, no test is yet available that would deal effectively with the differential diagnosis for an infant less than 12 months of age. Scales and instruments available at this time have limitations in the perspective of a dynamic evaluative approach, which identifies factors relating to significant risk for disabilities in order to utilise variables that could be followed to make an early determination of specific developmental abnormality (Hall, 2009; Himpens et al., 2010). However, these instruments play an important role as a basis for referral for full evaluation. Although some tests may provide important information regarding the functioning level of the infant that may be used as a baseline (Palmer, 2004). 


\subsection{Pathophysiology and Clinical Presentation}

Cerebral palsy is divided into five types depending on the area of insult in the brain and its clinical presentation on the body as summarized in the table below.

Table 1.1 Pathophysiology and clinical presentation of CP.

\begin{tabular}{|c|c|c|c|c|c|}
\hline \multirow[t]{2}{*}{ Diagnosis } & \multicolumn{3}{|c|}{ Pathophysiology } & \multirow{2}{*}{$\begin{array}{c}\text { Clinical } \\
\text { Presentation }\end{array}$} & \multirow[t]{2}{*}{ Percentage } \\
\hline & $\begin{array}{l}\text { Time of } \\
\text { insult }\end{array}$ & Area of insult & Type of insult & & \\
\hline Hemiplegia & $\begin{array}{c}\text { Neonatal } \\
\text { Period ( } 34 \\
\text { weeks or } \\
\text { later, } \\
\text { (Taylor et al, } \\
2009) \text { ) }\end{array}$ & $\begin{array}{l}\text { Central white } \\
\text { matter in the } \\
\text { Lateral } \\
\text { ventricle } \\
\text { (Scherzer, } \\
2000)\end{array}$ & $\begin{array}{l}\text { Placental embolism } \\
\text { or Enlargement of } \\
\text { one lateral ventricle } \\
\text { due to haemorrhage } \\
\text { leading to } \\
\text { leukomalacia } \\
\text { (Miller, 2005) }\end{array}$ & $\begin{array}{l}\begin{array}{l}\text { Unilateral } \\
\text { (one-sided) }\end{array} \\
\text { hemiparesis } \\
\text { and spasticity } \\
\text { seen more in } \\
\text { the upper limb } \\
\text { (Kuban et al., } \\
\text { 2008) may be } \\
\text { accompanied } \\
\text { by epilepsy } \\
\text { depending on } \\
\text { the severity, } \\
\text { intellectual } \\
\text { deficits in 50\% } \\
\text { of cases } \\
\text { (Miller, 2005) }\end{array}$ & $\begin{array}{c}30 \% \text { of } \mathrm{CP} \\
\text { cases }\end{array}$ \\
\hline Diplegia & $\begin{array}{c}\text { Prenatal } \\
\text { period } \\
\text { correlated } \\
\text { with factors } \\
\text { during } \\
\text { delivery } \\
\text { (Scherzer, } \\
2000) .\end{array}$ & $\begin{array}{c}\text { Posterior } \\
\text { external angle } \\
\text { of lateral } \\
\text { ventricles } \\
\text { (Miller, 2005) }\end{array}$ & $\begin{array}{l}\text { Periventricular } \\
\text { Leukomalacia and } \\
\text { Intraventricular } \\
\text { haemorrhage } \\
\text { (Scherzer, 2000). }\end{array}$ & $\begin{array}{l}\text { Increased tone } \\
\text { in lower limbs } \\
\text { following a } \\
\text { hypotonic and } \\
\text { lethargic } \\
\text { neonatal } \\
\text { period. Visual } \\
\text { difficulties and } \\
\text { strabismus. } \\
\text { Hypertonia } \\
\text { leads to } \\
\text { scissoring due } \\
\text { to increased } \\
\text { tone in } \\
\text { adductors of } \\
\text { lower limbs } \\
\text { followed by a } \\
\text { stage of hip } \\
\text { and knee } \\
\text { flexion with }\end{array}$ & $\begin{array}{c}22 \% \text { of } C P \\
\text { cases }\end{array}$ \\
\hline
\end{tabular}




\begin{tabular}{|c|c|c|c|c|c|}
\hline & & & & $\begin{array}{l}\text { internal } \\
\text { rotation of the } \\
\text { legs in } \\
\text { standing } \\
\text { (Himmelmann } \\
\text { et al., 2007). }\end{array}$ & \\
\hline Quadriplegia & $\begin{array}{l}\text { Prenatal } \\
\text { Perinatal } \\
\text { Postnatal } \\
\text { (Marret et } \\
\text { al., 2013) }\end{array}$ & $\begin{array}{c}\text { *Cortical } \\
\text { *Subcortical } \\
\text { Lesion } \\
\text { *Brainstem } \\
\text { *Basal Nuclei } \\
\text { (Marret et al., } \\
\text { 2013) }\end{array}$ & $\begin{array}{l}\text { *Brain } \\
\text { malformation } \\
\text { *Encephalomalacia } \\
\text { *Infections of CNS } \\
\text { *leukomalacia } \\
\text { (Marret et al., 2013) }\end{array}$ & $\begin{array}{l}\text { *Bilateral } \\
\text { spasticity } \\
\text { *Intellectual } \\
\text { deficit } \\
\text { *Microcephaly } \\
\text { (Himpens et } \\
\text { al., 2010) }\end{array}$ & $\begin{array}{c}25 \% \text { of } \mathrm{CP} \\
\text { cases }\end{array}$ \\
\hline $\begin{array}{l}\text { Dyskinetic } \\
\text { CP }\end{array}$ & $\begin{array}{c}\text { Perinatal } \\
\text { (Reddihough } \\
\text { \& Collins, } \\
\text { 2003) }\end{array}$ & $\begin{array}{c}* \text { Central grey } \\
\text { Nuclei } \\
\text { * Pontosubicular } \\
\text { necrosis } \\
\text { (Reddihough \& } \\
\text { Collins, 2003) }\end{array}$ & $\begin{array}{l}\text { *Hyperbilirubinemia } \\
\text { and hypoxia in } \\
\text { preterm infants } \\
* \text { Severe Asphyxia } \\
\text { * classification of } \\
\text { Basal Ganglia } \\
\\
\text { (Reddihough \& } \\
\text { Collins, 2003) }\end{array}$ & $\begin{array}{l}\text { Bilateral quick } \\
\text { jerky, } \\
\text { involuntary } \\
\text { movements } \\
\text { and shifts of } \\
\text { muscle tone } \\
\text { due to } \\
\text { emotional } \\
\text { stimuli } \\
\text { accompanied } \\
\text { by } \\
\text { Asymmetrical } \\
\text { tonic neck } \\
\text { reflex with } \\
\text { some degree } \\
\text { of spasticity } \\
\text { (Miller, 2005, } \\
\text { Panteliadis, } \\
2011 \text { ). } \\
\text { Symptoms } \\
\text { after } 5 \text { months } \\
\text { of age up to } \\
\text { the age of } 2 \\
\text { years Loss of } \\
\text { standing and } \\
\text { ambulation } \\
\text { due to the } \\
\text { inefficient } \\
\text { control of } \\
\text { trunk muscles. } \\
\text { Drooling with } \\
\text { affected } \\
\text { speech, with } \\
\text { normal } \\
\text { intelligence }\end{array}$ & $\begin{array}{c}5 \% \text { of } \mathrm{CP} \\
\text { cases }\end{array}$ \\
\hline
\end{tabular}




\begin{tabular}{|c|c|c|c|c|c|}
\hline & & & & $\begin{array}{l}\text { (Himpens et } \\
\text { al., 2010). }\end{array}$ & \\
\hline Ataxic CP & $\begin{array}{c}\text { Prenatal } \\
\text { (Sankar \& } \\
\text { Mundkur, } \\
2005))\end{array}$ & $\begin{array}{l}\text { Cerebellum } \\
\text { (Sankar \& } \\
\text { Mundkur, } \\
\text { 2005)) }\end{array}$ & $\begin{array}{l}\text { *Parenchymal } \\
\text { haemorrhage } \\
\text { leading to dysplastic } \\
\text { lesions of atrophy or } \\
\text { destruction } \\
\text { (Reddihough \& } \\
\text { Collins, 2003, } \\
\text { Himpens et al., } \\
2010 \text { ).) }\end{array}$ & $\begin{array}{l}\text { *Floppiness } \\
* \text { Truncal } \\
\text { oscillation } \\
\text { * Tremor } \\
\text { Affecting the } \\
\text { upper or lower } \\
\text { extremities or } \\
\text { both. } \\
\text { *Shows high } \\
\text { intellectual } \\
\text { deficit } \\
\text { variation. } \\
\text { (Panteliadis, } \\
\text { 2011). }\end{array}$ & $\begin{array}{l}10-15 \% \text { of } \\
\text { CP cases }\end{array}$ \\
\hline
\end{tabular}

\subsubsection{Hemiplegia}

Hemiplegic $\mathrm{CP}$ is the unilateral motor disability usually caused by a lesion during the neonatal period (Table 1.1). Its incidence has either been stable or decreasing throughout Europe in the last 20 years. More than 25 percent of hemiplegic cases have been born preterm (Taylor et al., 2009). Studies that investigate the neuropathology of hemiplegic CP are limited, and they only infer investigation from imaging findings (Miller, 2005). Prenatal or perinatal cystic softening of the middle cerebral artery is accounted to be common, but the cause has not yet been identified, although placental embolism is suspected (Miller, 2005). Another common factor would be subcortical lesion seen as an enlargement of one lateral ventricle in preterm infants, leading to periventricular leukomalacia (Fig 1.1) (Miller, 2005). 


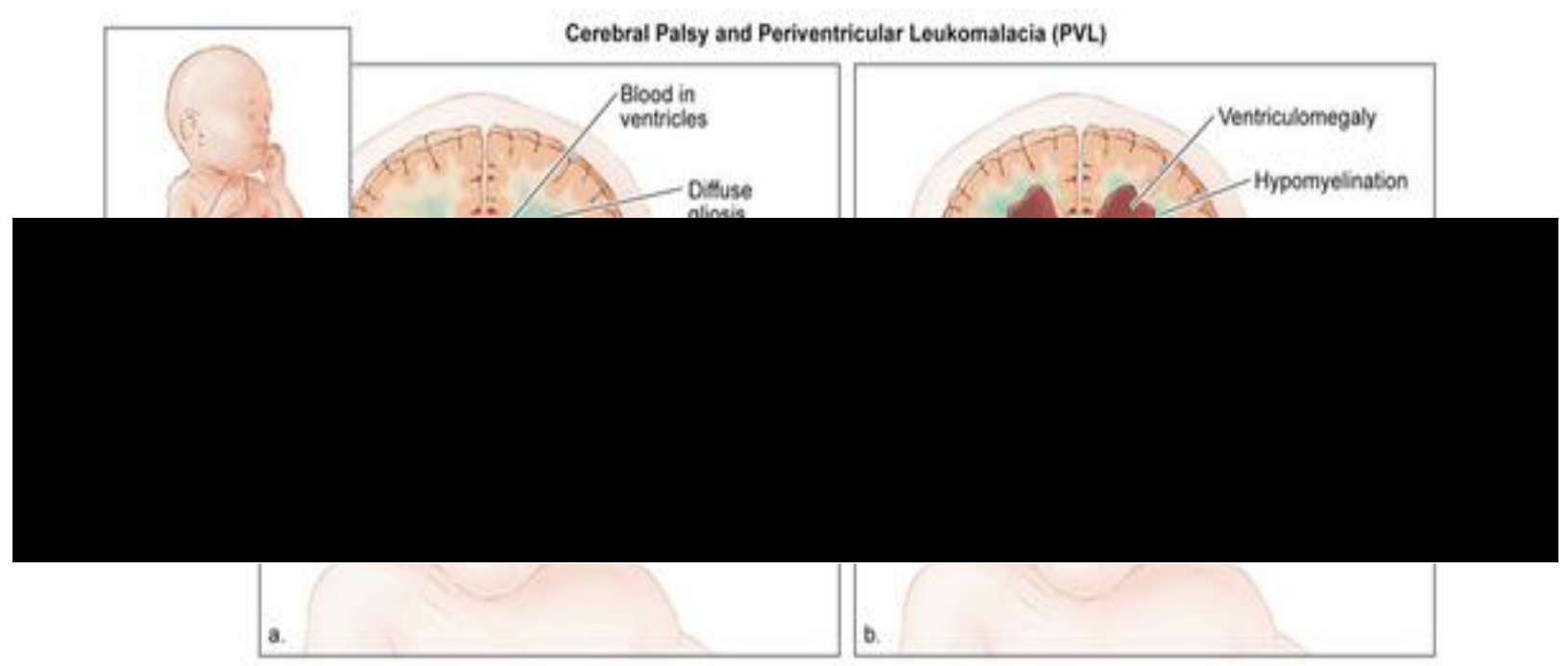

Figure 1.1 neonatology.ucsf.edu/specialized-care/cerebral-palsy.aspx (2016) The anatomical presentation of periventricular leukomalacia [Illustration]. Retrieved Feb 2016 from http://neonatology.ucsf.edu/specializedcare/cerebral-palsy.aspx

The ventricular dilatation seen in images of these cases is due to the loss of axons secondary to cortical cell destruction (Miller, 2005). Changes due to hemiplegic CP are seen in various outcomes in $\mathrm{CT}$ images such as maldevelopment, periventricular atrophy, cortical/subcortical atrophy, miscellaneous and even normal images (Miller, 2005). Some studies referred periventricular atrophy to be acquired before 34 weeks' gestation while cortical lesions of vascular origin are acquired later (Maclennan, 1999; Taylor et al., 2009). Hemiplegia is caused by haemorrhagic lesions, whether they are haemorrhagic leukomalacia, periventricular haemorrhagic (Fig 1.2) infarction or disruption of the central white matter due to blood from a ventricular haemorrhage (Scherzer, 2000). 


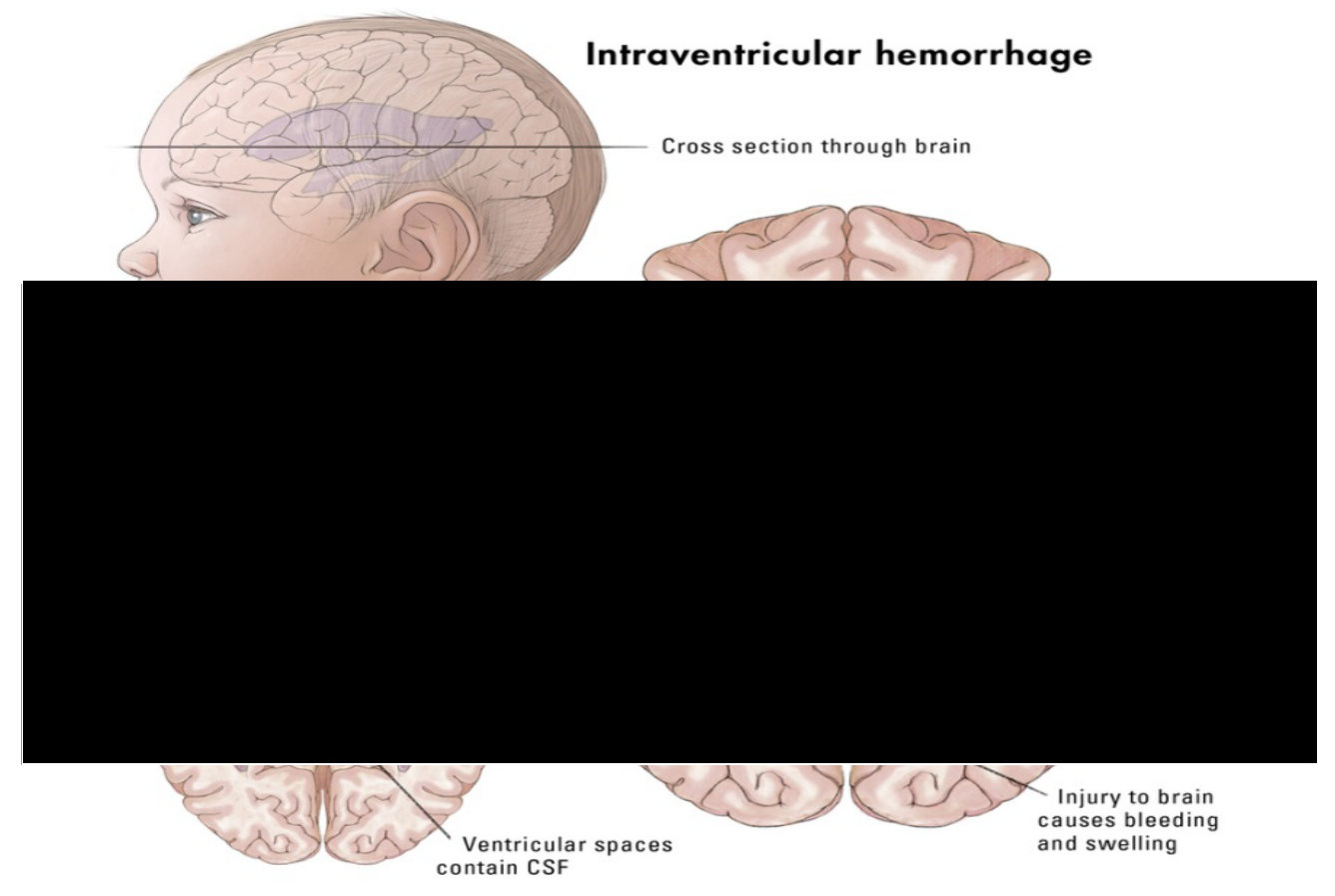

Figure 1.2 kin450-neurophysiology.wikispaces.com (2016) Intraventricular Hemorrhage [Illustration]. Retrieved Feb 2016 from https://kin450-neurophysiology.wikispaces.com/

Lesions, which involve the cortex similar to the cystic softening, are usually associated with epilepsy and mental deficit compared to the subcortical lesions (Scherzer, 2000). Predictions, however, based on CT images, are unreliable (Scherzer, 2000). The main characteristics of hemiplegia are unilateral hemiparesis and spasticity, with the weakness predominating the distal parts of the limbs. It is rarely diagnosed at birth and silent intervals can continue until nine months of age (Hall, 2009; Kuban et al., 2008). Early manifestation can be seen by five months in the majority of cases as early hand preference with fisting and abnormal flexion positions of the elbow (Hall, 2009; Kuban et al., 2008). Diagnosis, however, can be late up to the age of 18 months. Milestones are generally delayed, however, hemiplegic cases in children usually walk at a normal age (Himmelmann et al., 2007).

Appearance of hemiplegia usually involves the upper limb more than the lower and is characterised by the abduction of the arm with a hyperextension of the fingers, which are widely apart. In cases that involve the lower limb, it is believed that their lesion is related to hydrocephalus or periventricular leukomalacia, which involves the periventricular pyramidal 
fibres commanding the lower limbs (Miller, 2005). In congenital hemiplegia, no facial lesions are involved apart from a mild deficit localised to the VII nerve compared to total facial palsy in the acquired hemiplegia. Growth and development also differ in the affected side of these cases (Miller, 2005).

Physical signs include spasticity and associated movements that overshadow weakness, increased tendon reflexes, Babinski and Rossolimo signs, Cortical sensory abnormalities as well as the impaired visual field. Epilepsy is another major complication that varies in frequency according to the type of referral and is successfully medically treated in 80 percent of cases (Miller, 2005). Intellectual deficits were found in up to 50 percent of cases and are highly correlated with epilepsy and the severity of hemiplegia (Miller, 2005).

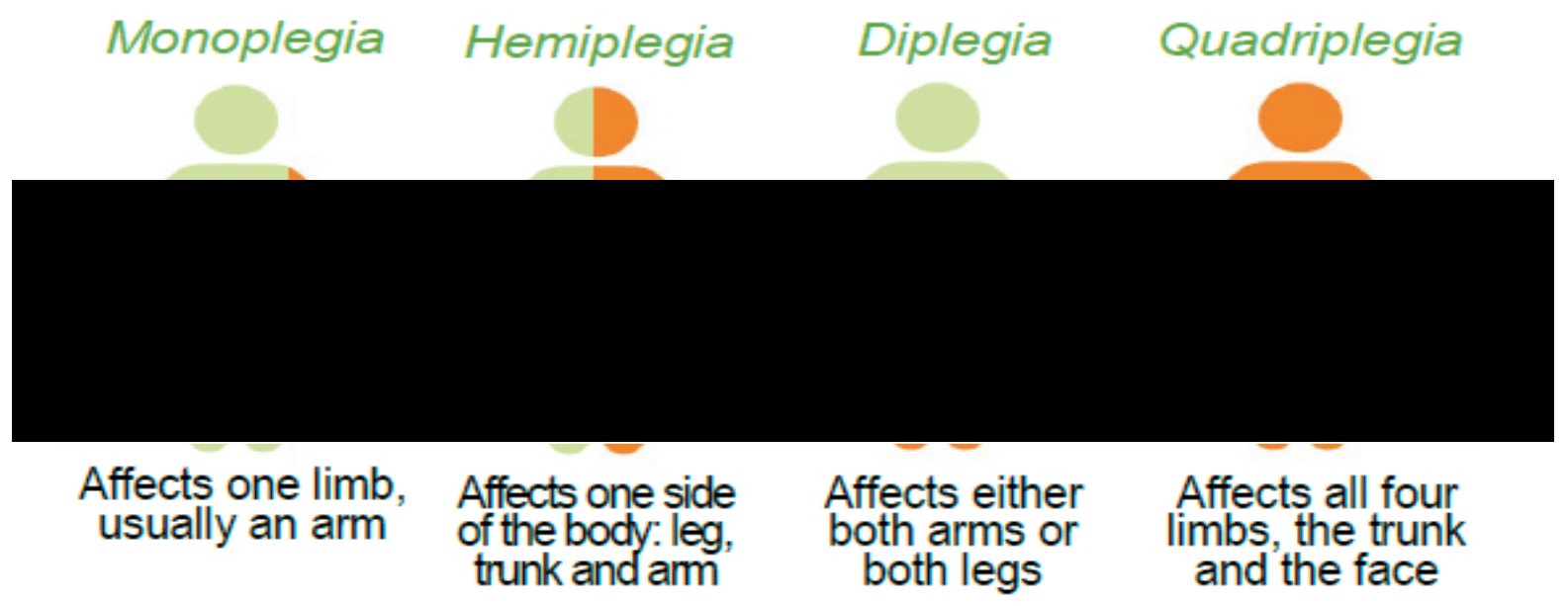

Figure1.3 Slidershare.net. (2016) Topographical presentation of Cerebral Palsy. [Online Illustration] Retrieved December 2016 from: http://www.slideshare.net

\subsubsection{Diplegia}

Diplegia is the term for describing the form of $\mathrm{CP}$ which involves the lower limbs and is considered the most common type of CP (Scherzer, 2000). Spastic diplegia is referred to as the incidence of preterm delivery with LBW below 1500 grams, in addition to the history of reproductive failure in mothers in some cases (Scherzer, 2000). This refers to prenatal factors that might correlate with factors during delivery as well. Periventricular lesions are the predominant location of damage in preterm babies (Scherzer, 2000). 
Intraventricular haemorrhage, especially when followed by dilatation, is also a common cause of diplegia. The most common lesion, however, that might cause spastic diplegia, is periventricular leukomalacia (Scherzer, 2000). These lesions are located along the external angle of the lateral ventricles, therefore, damaging the fibres from the internal aspect of the hemisphere and includes the motor fibres of the lower limbs (Scherzer, 2000). Furthermore, the location of the leukomalacia along the posterior part of the lateral ventricles gives rise to visual difficulties and strabismus by interrupting the optic radiation (Scherzer, 2000). In severe cases of diplegia, ultrasound images of preterm infants show cavitated leukomalacia (Miller, 2005). On the other hand, in many cases that are not as severe, no abnormal images have been seen by ultrasound or even computed tomography (CT).

Clinical feature of diplegia (Fig 1.3) includes increased muscle tone in lower extremities, hypotonia, lethargy, and feeding difficulties during the neonatal period (Miller, 2005). Hypotonia might persist in the majority of diplegic cases for up to 12 weeks of age, causing suspicion (Marret et al., 2013). This is followed by some involuntary exaggerated movements whenever the child's position is changed, which is known as the dystonic stage. Scissored extended legs are then seen due to spasm in the adductor muscles. This is followed by a stage of predominant hip and knee flexion with internal rotation of legs in standing (Himmelmann et al., 2007). Severity would vary and shown as overt foot walking or no walking at all in severe cases due to lack of balance, truncal hypotonia or contractures (Himpens et al., 2010).

Deep tendon reflexes are hyperactive in all extremities and pyramidal signs are present, and easily identified. In many cases, the lesion is asymmetrical and can even be seen as a monoplegic manner that represents the consequence of unilateral leukomalacia (Marret et al., 2013). In rare cases, the associated dystonia will develop after several years of stable symptoms. Epilepsy is very uncommon but might be seen in some rare conditions, strabismus, on the other hand, is very common (Marret et al., 2013). In more than 60 percent of cases, normal or borderline intelligence is preserved. However, the increase in the survival of very low birth weight infants is showing an increase in cases of diplegia with more intellectual deficits, epilepsy and marked motor disability (Marret et al., 2013).

Another type of diplegia would be ataxic diplegia. It accounts for $5-7 \%$ of cases of CP and is usually congenital. It usually has prenatal causes, however, it might also be caused by 
perinatal asphyxia. It is considered the acquired defect following infantile hydrocephalus. Ataxic diplegia is shown initially as hypotonia, which progresses to spasticity and increased tendon reflexes. By the first year of age, the child will start to show tremor and titubation in the sitting position. The use of arms is usually difficult due to ataxia and standing, and walking might not be possible independently (Himmelmann et al., 2007). As for the mental level, it is usually normal in more than 65 percent of cases.

\subsubsection{Quadriplegia}

Tetraplegia or quadriplegia is the most severe form of CP. It might also be called dystonic and tetraplegia dystonia in some literature, as the difference between dystonia and tetraplegia is in some cases, is difficult to see (Himpens et al., 2010). Bilateral spasticity is the main characteristic predominating in the upper limbs in addition to the bulbar muscle paralysis associated almost always with severe intellectual deficit and microcephaly (Himmelmann et al., 2007; Himpens et al., 2010). Children with tetraplegia present only 25\% of CP cases, however, they are totally dependent and pose the most difficult problems of care, feeding and prevention of deformity (Himpens et al., 2010). Speech absence and gross dysarthria, in addition to major problems regarding feeding and prevention of aspiration, leaves the infants totally helpless. They show a very poor outlook and most affected infants do not develop beyond a neonatal stage (Himpens et al., 2010).

The causes could be prenatal, perinatal or even postnatal, with a high incidence of brain malformation (Table 1.1). Destructive prenatal lesions such as multicystic encephalomalacia and hydranencephaly are also common in addition to the infections of the CNS such as herpes simplex virus encephalitis. In some cases, there is damage to the brainstem and basal nuclei in association with cortical and subcortical lesions as well as the development of thalamus calcification in other cases (Marret et al., 2013). 


\subsubsection{Dyskinesia}

Dyskinetic $\mathrm{CP}$, also known as athetoid or extrapyramidal $\mathrm{CP}$, is represented by the inability to organise and properly execute intended movements or automatic coordinate movements or maintain a posture (Table 1.1) (Miller, 2005). Dyskinetic forms of CP are well defined regarding the etiological and clinical points of view (Miller, 2005). On the other hand, confusion exists with respect to the terms used. Many different terms have been used in the literature to describe the lesion and its movements similar to athetosis, chorea, dystonia, and dyskinesia (Miller, 2005). This, however, does not reflect any practical necessity as variable abnormal movements, in this case, are usually due to the same cause and present in the same patient (Miller, 2005). In addition to the major disability caused by the symptoms of dyskinesia, primitive motor patterns persist with the patient such as the asymmetrical tonic neck reflex with some degree of spasticity (Panteliadis, 2011).

Dyskinetic CP can be further divided into two subgroups, the hyperkinetic subgroup and the dystonic subgroup that are caused by different aetiology and correspond to a distinct outcome. The hyperkinetic subgroup is featured by massive purposeless involuntary movements that may consist of a variety of types (Jameson et al., 2010). Athetosis is the slow writhing movement of the distal parts of the limbs associated with fingers in hyperextension. As for choreic movements, they are known as the quick jerky movements that involve the more proximal muscles (Scherzer, 2000). Dystonic posture, on the other hand, describes the tonic contractures that involve the whole limb or axial muscles, which are also of slow and writhing nature. Other hyperkinesia that might be associated includes tensions myoclonus or ataxia (Scherzer, 2000). All of these previous movements are induced by attempted movements or efforts to control posture. A feature of dyskinetic patients would be their inability to restrict movements to the intended pattern and location, resulting in an overflow of movements to antagonist and agonist muscles that should not have been involved (Krigger, 2006).

The dystonic subgroup, on the other hand, is characterised by abnormal shifts of general muscle tone due to emotional stimuli (Krigger, 2006). In these cases, there is always a strong primitive reflex that interferes with the effort to perform any voluntary movement. Spasticity may be associated in some cases. Perinatal factors are responsible for most of the cases compared to other types of CP. Prenatal and postnatal factors may also be a cause for a 
much smaller percentage (Reddihough \& Collins, 2003). Hyperkinetic cases are seen mostly in preterm infants with hyperbilirubinemia and hypoxia and may also be seen in term infants with a normal birth weight that has suffered severe asphyxia or even in LBW infants with hypoxia. No specific aetiological factor is necessary for dyskinetic CP (Reddihough \& Collins, 2003). Some show calcification of the basal ganglia.

The main pathological characteristic seen in dyskinetic CP is remarkably selective involvement of the central grey nuclei with associated atrophy and sclerosis (Reddihough \& Collins, 2003). While in preterm infants, the lesion is seen less clearly individualised and seen usually with pontosubicular necrosis and other brain damage (Reddihough \& Collins, 2003; Scherzer, 2000). The lesion is seen as a marbled appearance of the basal ganglia, especially the striatum and thalamus, which is due to the abnormal deposition of myelin in association with diffuse gliosis. It is also common to have associated lesions of the ulegyrias of the central convolution (Scherzer, 2000). Only global atrophy is occasionally seen in imaging, which is rather disappointing as an investigation (Krageloh-Mann, 2008).

Symptoms become noticeable only after five months of age and may not show in full before the age of two years (Himpens et al., 2010). Early symptoms appear similar to those of a dystonic stage of diplegia involving mainly the trunk and lower limbs. An open mouth is the first sign of further evolving dystonic CP (Himpens et al., 2010). Other symptoms follow by showing normal or no tone at rest. Inefficient control of trunk muscles is usually the reason of loss of standing and ambulation abilities, but only a minority of patients are able to walk independently (Himpens et al., 2010; Reddihough \& Collins, 2003). Speech is affected due to the Bucco-pharyngo-laryngeal muscles being involved or due to hearing loss in some cases (Scherzer, 2000). Drooling is also associated. Intelligence is either normal or within the dull normal range. In many cases, the vision is affected, and strabismus is also very common (Himpens et al., 2010).

\subsubsection{Ataxia}

Ataxic CP is another CP form that assembles 10-15 percent of cases of CP that is mostly of a congenital nature (Sankar \& Mundkur, 2005). It is differentiated from ataxic diplegia by its prominent cerebellar symptoms that can only be clearly seen by the walking 
age of 1 or even 2 years. It is usually (25\%) caused by prenatal factors or genetic factors that are related to other syndromes (Sankar \& Mundkur, 2005). In $41 \%$ of cases, no cause has been found (Sankar \& Mundkur, 2005). Other studies had found hereditary causes leading to disequilibrium syndrome or hemispheral cerebellar atrophy related to hypoplasia of the vermis and hemisphere. However, the relation of these syndromes to ataxic CP is still not very determined (Himpens et al., 2010; Redihough \& Collins, 2003). Acquired factors of ataxia, on the other hand, include the sequela of cerebellar parenchymal haemorrhage (Table 1.1) (Sankar \& Mundkur, 2005). Dysplastic lesions of either destructive or atrophic nature can be responsible for ataxic CP, which pathology is imperfectly known, yet even though dysplastic abnormality seems to predominate (Scherzer, 2000). Most children show symptoms of floppiness and docility from early in life. Truncal oscillation on attempted sitting is another clear symptom with tremor when reaching for objects in addition to abnormal elevation of the feet in four-point walking (Panteliadis, 2011). Examination shows general hypotonia. Ataxic symptoms differ from one child to the other, which affect the upper or lower extremities or both. Pyramidal tract signs are only seen in severe cases while in most cases, they are able to walk at the age of 4 despite the frequent falling (Panteliadis, 2011).

Intellectual deficit is seen in variation within the population of ataxic $\mathrm{CP}$, however, the severe intellectual deficit is rare. Some cases attend normal school and adjust to their disability. The major challenge is adjusting the tone and maintaining control of posture in standing (Panteliadis, 2011).

\subsection{Prognosis of CP Cases}

Studies have suggested the age of 3 for a severely affected child to reach $90 \%$ of their motor potential and the age of five for the less severely affected (Miller, 2005). Predicting a child's motor abilities usually depend on observational factors of the child's prognosis at certain ages and milestones (Campbell et al., 2006). It is predicted that children who acquire sitting up to the age of 24 months have a better chance of walking, given that all the children that might be able to walk do walk at the age of 8 (Campbell et al., 2006). Prognosis may also depend on the type of CP. Predicting mobility through sitting abilities remain the most 
common predictor of ambulation, although other factors such as a child's neonatal variables, clinical types of $\mathrm{CP}$, and primitive reflexes and reactions may also have an effect on the child's prognosis (Campbell et al., 2006, Miller, 2005). Rigid or hypotonic types of CP cases are less likely to walk, moreover, an increase in tonic neck reflexes may also result in less likelihood of walking (Miller, 2005). Hemiplegia, on the other hand, have a good prognosis of ambulation (Campbell et al., 2006). Other factors that have been categorised into groups are considered variables and may be evaluated by therapists who are influential in controlling the number of motor abilities of children with CP. These factors are seen as 1) primary impairments such as muscle tone and movement patterns. 2) Secondary impairments such as force production and endurance. 3) Personality characteristics. 4) Family factors. Education and employment, although received the scant attention of prediction in the literature had shown increased rates over the years (Campbell et al., 2006). This is due to the developing efforts and advances that have access to technology, environmental access and supportive legislation. Other factors concerning limited physical involvement, good home support, education, vocational training, and good cognitive skills, all have a positive prognosis on successful employment for adults with CP. Despite the positive change in employment of adults with CP, a percentage of about $60 \%$ are still dependent on caregivers and elderly parents (Campbell et al., 2006).

\subsection{Management of CP}

\subsubsection{Physiotherapy Management}

In the management of a $\mathrm{CP}$ infant, a path for treatment is made according to the functional consequences that the infant presents, as well as the deficits arising from the symptoms identified in the evaluation. For example, persistent primitive motor patterns and hypotonia will definitely have consequences on posture, movement and motor development (Erriksson et al., 2006). Daily care procedure in that matter should be given as much attention as treatment, given that it has a major effect on the child's interaction with the environment (Miller, 2005). Management of a child should cover handling positioning and daily care in order to support the treatment plan made for the child by the therapist (Miller, 2005). It should include awareness of the child's special requirements for carrying, adequate support either of posture or motor actions and supervision needed for tone, motor behaviour and 
limitations (Miller, 2005; Panteliadis, 2011). Proper positioning of a child for any task in daily living is considered essential in preventing complication and limitation in movement as well as promoting normal motor development (Bower, 2001).

As the treatment approaches of therapy have been evolving, the trend towards early diagnosis intervention had shifted the attention away from orthopaedic surgical approaches and into global remediation of deficits by focusing on strengths, use of technology and cognitive needs (Miller, 2005). Physiotherapy approaches that are available at this time have been evolving in the past 50 years. However, limited evidence exists on one approach being more superior to another, even though some show more popularity compared to others (Darrah et al., 2004). Therapy should be offered as the earliest treatment intervention of a developing child (Mahoney et al., 2004).

Early intervention includes multidisciplinary services that are provided to the child from birth to five years of age, to promote child health and wellbeing with the goal of minimising developmental delays and, prevent functional deterioration, remediate existing disabilities (Hospers \& Algra, 2005). These goals may only be achieved through individualized developmental, educational and therapeutic services. Although the most effective early intervention has been identified to be in the NICU and infant age. However, it has been found that developmental programs in the first 2 years of life have shown to provide a positive effect in motor development by stimulating the child's exploration of active motor behaviour (Hospers \& Algra, 2005). According to that approaches now, it has influenced the early intervention through general developmental programs, education, early learning and social maturation of the child, in addition to, support and direct families by providing treatment as a total educational effort, which enables contact with other infants and families whenever possible (Blair \& Whalsten, 2002).

As the child grows and restrictions in range of motion and functional dependence appear, an orthosis will be recommended in hope to overcome the deformity forces, prevent contractures and stabilize posture and gate, given that orthosis do have an effect on sensation in addition to motor functioning (Miller, 2005). Inhibiting casting can also be used alternatively or together with orthosis to promote weight bearing, by reducing contractures especially for hemiplegic patients (Miller, 2005; Panteliadis, 2011). 
Physiotherapy is one of the common treatments that play a central role in the management of CP. It manages the condition by providing an improvement of function, movement and overall use of child potential (Antilla et al., 2008). It also promotes, maintains and restores physical, psychological and social wellbeing. An important role of physiotherapists is educating the parents' of children with CP regarding the handling and some preventive techniques in addition to mobile devices, an aspect that is highly variant and depends on the child's needs and the parents' level of involvement as well as the level of society's overall awareness level. In the literature, some gaps have been found in investigating and supporting the level of family involvement in the management of children with CP. Therefore, a pilot study will be implemented in Kuwait to identify the effects of family education in supporting the outcomes of clinical physiotherapy.

As any other treatment physiotherapy approaches require the need to be supported by evidence. Effectiveness of many approaches has been assessed through many systematic reviews. Approaches include strength training, conductive education, focused induced therapy such as constraint therapy, postural control, passive stretching, hydrotherapy, hippotherapy, and the most common treatment neurodevelopmental therapy or as known as Bobath therapy. The literature lacks high-quality research resulting in difficulty to determine the effectiveness and efficiency of the available treatment (Antilla et al., 2008). Therefore, it must be investigated if the effectiveness of current interventions such as the Bobath approach may be improved by delivering it with a supportive method such as a tailored and supervised home intervention through a narrative review. Results supporting these assumptions must then be published in various forms including journal, posters, and scientific conferences in order to expand research in this specific area and furthermore, change practice.

Paediatric physiotherapy used to follow an orthopaedic approach which consists of an alignment of joints and muscles through splints and furthermore, encourages normal milestones. This developed into the efforts to modify tone and movement to achieve an improved daily function that indicates the rise of the neuroplasticity evolution and nervous system functioning. This has changed again after realising that changing the tone and movement is not equivalent to better functional outcomes, which led to providing treatment as functional tasks to promote the use of its effect in daily living in a different environment (Mayston, 2011). In the meantime, several new approaches have emerged and continue to do 
so. This has been raising a concern and challenge to the therapist, children and caregivers, to show which treatment to apply and which treatment is most effective.

Neuroplasticity is the term that identifies a range of neural responses from cellular and molecular mechanisms of synapse formation to cellular realignment or organisation of neural network, learning, or memory and behaviour (Mundkur, 2005). At Birth, the brain is very immature and highly dependent and is modified by experience (Mundkur, 2005, Pape, 2012). This can be explained in cases where children are born blind, the parts of the brain that normally processes visual information are rewired and come to process sounds, including language. In other cases, where children are born deaf, the areas responsible for processing sound come to process vision (Mundkur, 2005). The plasticity of the brain is maximal during the critical period of a child's lifetime that falls between birth and approximately 7 years of age. However, in older children, the end of the critical period does not mean the complete loss of experience modified synaptic plasticity, it rather becomes restricted and perhaps more difficult (Mundkur, 2005, Pape, 2012). The major types of plasticity in children are known as adaptive, excessive and impaired. Adaptive plasticity is the type which allows changes in neuronal circuitry and enhances a special skill with practice, which then compensates for injuries (Aisen et al, 2011, Pape, 2012). Adaptive plasticity is the main type that is targeted by physiotherapy in children with CP (Aisen et al., 2011, Pape, 2012). Impaired plasticity, on the other hand, refers to situations where genetic disorders disrupt molecular plasticity pathways similar to Coffin-Lowry syndrome (Aisen et al., 2011, Pape, 2012). The third type is excessive plasticity that can lead to disability through reorganisation of new maladaptive neuronal circuits that cause neurological disorders such as partial seizures following mesial temporal sclerosis or focal dystonia (Aisen et al., 2011, Pape, 2012).

In cases where a body part is not in use, the representation area of this part in the brain shrinks and vice versa (Burtner et al., 2014). When a specific part is continuously and widely used, the area representing that part in the brain is enlarged. In the case of a brain injury that damages the representation of a part of the body in the primary motor cortex, plasticity will permit reorganisation to restore a representation (Burtner et al., 2014). While the primary motor cortex is the main and most powerful contributor to the corticospinal tract, the premotor cortex is also a secondary less powerful contributor. However, the stimulation thresholds of the premotor cortex are higher than the primary motor cortex, therefore, it could be the source of supraspinal control signals (Hallett, 2005). A prolonged period of nerve 
stimulation will increase the excitability of related muscle representation in the motor cortex, which is the principle of physiotherapy approaches of similar constraint-induced movement therapy and bimanual therapy (Hallett, 2005). Additional lines of evidence support the ability of adjacent surviving neurons to take over the function of the damaged cortical areas (Ridding et al., 2001, Reid et al., 2015). This happens by the cortical areas whose primary activity is to control a group of muscles and take over the control of other muscles whose corticospinal projections were damaged (Ridding et al., 2001, Reid et al., 2015). This could be facilitated by the application of specific peripheral stimulation paradigms that increase the size and strength of the cortical projection of the weakened muscles (Ridding et al., 2001, Reid et al., 2015).

Novak et al. (2013) report that Bobath is the most common approach of physiotherapy used to manage the physical function deficits resulting from CP. In the literature, however, little evidence exists to support its effectiveness in the treatment of children with cerebral palsy. The Bobath approach also known as Neurodevelopmental Therapy (NDT) was first created by the Bobaths in the early 1940s with a concept of inhibiting abnormal reflexes and movements only, to encourage normal postural reactions and movement patterns (Butler \& Darrah, 2001). This is done by providing the child with the proper physical handling during movement passively, to allow the child improved sensory-motor feedback. Gradual withdrawal of support is done as the child's posture and movement begin to improve (Bobath, 1992). The approach initially focused on muscle tone, reflexes and abnormal movement patterns, postural control, sensation, perception and memory as sensory and motor components (Butler \& Darrah, 2001) using the normal development as the guidance of treatment goals.

As the knowledge of neuroscience developed and progressed, the approach is needed to change, improve and evolve in order to replace some treatment basis that was ineffective or shown has effected such as inhibiting postures (Raine, 2007). As they showed no effect in motion, the focus was turned to inhibiting abnormal patterns of movement as the child was moving, which was more practical and beneficial for the child. Assumptions of the child translating therapeutic experience into the voluntary movement were then changed by embracing the child's disability and giving the child the control of their movement and balance and efficiency of movement (Raine, 2007), rather than trying to push the child to achieve normal developmental levels of movement. These changes were as a result of not 
seeing changes carried over the child's active life in their home environment, leading to the conclusion that a treatment approach should be given in actual settings that a child is living in either at home or playing and learning environments (Raine, 2007). According to the recent changes, there was controversy in the literature regarding if the current Bobath practice is valid, given that its concept is no longer influenced by hierarchical models of motor control but are practical changes that were made in the field according to feedback retrieved from patients' prognosis. The concept now follows the system model of motor control indicating that the CNS is only one of many systems controlling movement.

A supporting modality of treatment would be orthotics and has a long history of prevention for the further complication of CP similar to contractures. However, orthotics are now being promoted as part of a total treatment approach, otherwise considered as passive, restrictive and without the benefit (Miller, 2005).

Static orthosis promotes maintenance of an achieved range of motion while dynamic orthosis assists in alignment of joints, which supports movement (Fig 1.4). Casts can also be used in increasing range of motion and decreasing tone (Papavasiliou, 2009).

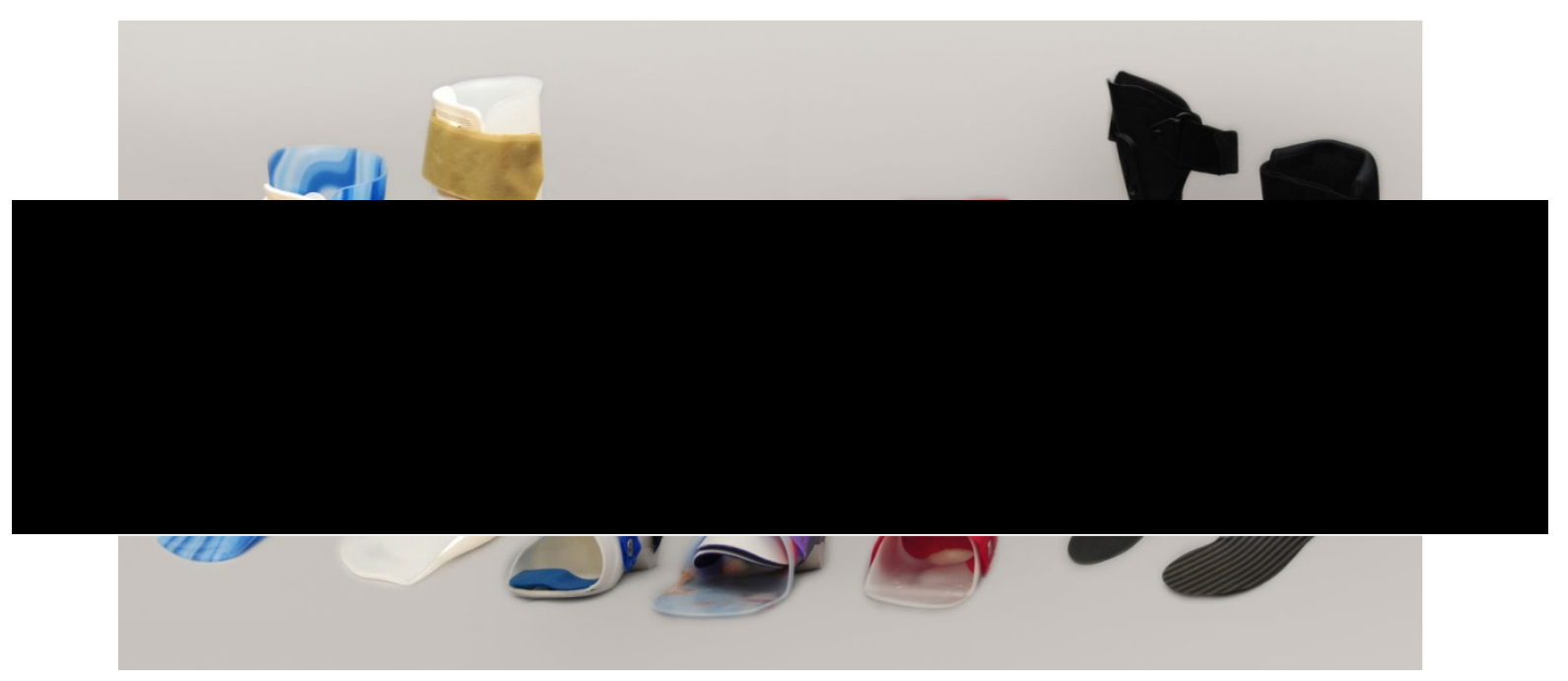

Figure.1.4 Getdomainsvids.com. (2016) Various types of Knee Ankle Foot orthosis that depend on the different needs of each case.[Meme] Retrieved Feb 2016 from:

http://www.getdomainvids.com/keyword/orthotic\%20braces/

Treatment and management of a child with CP should involve the child as a whole including the family, the home environment and the school. Many approaches exist in treating the child with cerebral palsy, some are widely used, although they are still 
investigated and questioned. Research is still needed for most if not all the treatments available for cerebral palsy, especially in the parameters of treatment delivery and the longterm effects of approaches. A systematic review will be created to investigate the effects of home treatment seen in previous literature.

The choice of treatment will mainly depend on available classification and measurement tools and most importantly, the need of the children and families in order to provide a tailored made intervention that fulfils all the requirements. The classification tools are used to classify the child's severity level depending on functional abilities and also provides and predicts the child's level of prognosis. After classifying the level of severity and predicting the level of prognosis of the child's functional prognosis, the physiotherapists are then able to set treatment goals of the treatment provided. In order to evaluate this procedure in Kuwait and identify the objectivity of goal setting and goal achievement a clinical audit is required to identify the level of efficiency in order to promote improvement in future practice. Studies in the literature tend to provide evidence regarding which approach is better than the other while there should be some agreement that there is no single way to provide treatment. Variation in the cases of children with cerebral palsy, based upon the classification of the level of severity, makes the generalisation of one treatment or specific parameters for another treatment rather difficult, if not impossible.

\subsubsection{Gross Motor Function Classification System}

The Gross Motor Function Classification System (GMFCS) was established to provide an objective classification of motor disability in CP children (Wood \& Rosenbaum, 2000). The concept of GMFCS was created from the GMFM and validated by Palisano et al., (2000). The GMFCS classifies the level of motor ability according to self-initiated movements during functioning in sitting and walking, it also predicts the future motor ability of the child with CP. Levels of the GMFCS are based on the abilities and limitations of the child with $\mathrm{CP}$ and are divided into five levels, starting with level I which includes children with the most independent motor ability to level VI which includes children with the least independent ability. The levels are also divided by age into a functional description of each age level that includes 1-2, 2-4, 4-6 and 6-12 years of age. Before the creation of the GMFCS, many classifications existed to support the diagnosis of cerebral palsy that included 
parts of the body that is most involved and ambulatory method, but none of those measurements was supported by validity or reliability reports. Therefore, a more objective classification system was required (Rosenbaum et al., 2008).

In 1994, a search began for the establishment of an alternative approach that would depend on the classification of functional abilities and limitations. Bearing in mind the performance of the motor function in everyday life, according to Rosenbaum et al., (2008), the key element for the creation of the GMFCS that would enhance the communication between the families and professionals. The GMFCS had shown evidence for the stability of results over repeated measurements on a long-term study basis (Palisano et al., 2000) when tested on a group of children with an average of 4 measurements per child showing very high test-retest reliability. The GMFCS had been confirmed to be very easy to use, thus relying on usual gross motor functions and clinical information that is routinely observed when assessing a CP child in addition to its excellent interrater reliability. The system does not require any special skills or procedures to use nor does it require any complicated information in order to be used in assessing the severity of CP level. The system allows therapists to compare $\mathrm{CP}$ cases with other treated by different therapists to ensure that the children compared have the same level of CP severity.

\subsubsection{Gross Motor Functional Measurement}

The Gross Motor Functional Measure (GMFM) is an observational method that depends on referenced criteria that have been developed to evaluate the level of mobility in children with cerebral palsy (Rosenbaum et al., 2008; Russell et al., 2000). In addition, the measurement of change in motor ability over time or change as a response to an intervention (Brunton \& Bartlett, 2011). At the age of five, a child with no motor delays is able to perform all items of the GMFM. The method contains 88 items (Appendix 1) that assess movements according to a 4 -point ordinal scale $(0=$ does not initiate, $1=$ initiate $<10 \%$ of activity, $2=$ partially completes $10 \%$ to $<100 \%$ of activity, $3=$ completes activity). GMFM is divided into five dimensions, which includes 1) lying and rolling, 2) sitting, 3) crawling and kneeling 4) standing, 5) walking, running and jumping. Administration of the GMFM may be found in Appendix 2. The GMFM88 had proven to be meaningful in evaluating the change in gross motor function (Brunton \& Bartlett, 2011). The GMFM88 was further developed after being 
extensively used in GMFM66 to improve the scaling of the measurement. After analysis to the GMFM88, the GMFM66 allowed the hierarchical structure of the items to be revealed (Brunton \& Bartlett, 2011). Russel et al., (2002) had suggested the use of the GMFM88 for children in level IV and V of the GMFCS which may detect more change especially in the lying and sitting category and the use of GMFM66 for children with a higher level of I-III which could be more sensitive to change in other categories. However, the study had also suggested that it is safe to use the GMFM88 when there is no computer available for using the GMFM66. The GMFM88 has demonstrated a very high level of intrarater and interrater reliability according to Russel et al., (2002). In addition, Drouin et al., (1996) had supported the tool's validity by reporting a strong correlation between gait speed and dimension D (standing) and E (walking, running and jumping). Other studies that included Russel et al. (2000) had assessed the validity of the GMFM on different levels of children with CP in addition to other cases that included acute head injuries as well as children who had no motor delays. The study had included the judgment of caregivers, treating therapists in addition to therapists who were unfamiliar and were not treating the children in order to confirm the validity of the measurements taken.

Another study (Damiano et al., 2002) had confirmed the validity of the GMFM when a strong correlation was seen between computerised gait analysis and scores of the GMFM. The tool should be performed only by a trained physiotherapist. This would ensure the reliability of the tool especially when it is not performed by the treating therapist, as personal therapists tend to provide a higher score when applied for their patients (Han et al., 2010). The GMFM shows more sensitivity to change in children of GMFCS level of I, II, and III compared to the level IV and V which might show less change in the scores of the measurement tool (Nordmark et al., 2000). The use of the GMFM has also shown some limitations that could affect its use. Children with motor disabilities that are considered moderate and function in the middle of the scale show a higher change in scores when compared with children that are either very mild or very severe. Another limitation of the tool is that children with different types of disabilities and motor abilities may achieve the same score (Russel et al., 2002). 


\subsubsection{Medical and Surgical Management}

Spasticity in CP cases is considered the main motor deficit and the most obvious. Medical treatment has emerged in the last several years but its results in treating or temporarily inhibiting spasticity are yet to be seen (Scherzer, 2000). It is also still in need of standardised indication of use in addition to consistency and uniformity. Botulinum toxin (Botox) is being used widely for the sake of reducing and delaying gastrocnemius spasticity in addition to postponing surgical intervention (Lukban et al., 2009). Similar effects have been seen in hip adductor muscles in addition to the reduction of subluxation in some cases (Scherzer, 2000). Although it has been widely used in the past years, its effect on the rapid change due to growth in children younger than three years raises some concerns, other factors such as pain bruising and weakness in the target muscle are considered as limiting factors for its use. It has been used with children as young as 4 months to treat torticollis in some countries but is still waiting to be approved for that age in the US (Scherzer, 2000). Other spasticity inhibitors include Baclofen that may be taken orally or intrathecally (Intrathecal Baclofen ITB) (Fig 1.5) and is being widely used for CP cases above the age of 4 despite the serious side effects that also include seizures (Scheinberg et al, 2006).

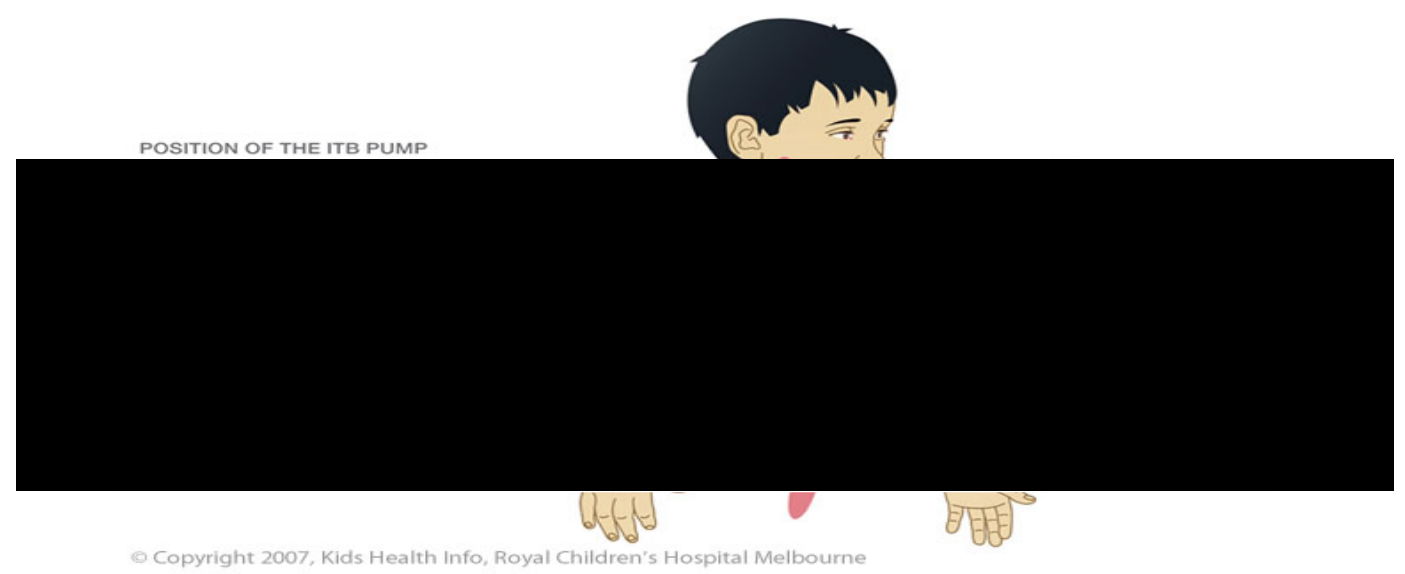

Figure.1.5 rch.org. (2016) Intrathecal Baclofen pump [Illustration]. Retrieved Feb 2016 from http://www.rch.org.au/kidsinfo/fact sheets/Intrathecal baclofen pictures/

Cases that exceed this age and develop problems similar to contractures and dislocations in severe cases might be in need of more invasive procedures such as contracture 
releases (Scherzer, 2000). Selective dorsal rhizotomy is another surgical procedure that identifies, then divides nerve rootlets that are associated with an abnormal motor response without harming the other rootlets that are functioning normally (Ailon et al., 2015). It is a procedure that has been getting attention recently and is being performed even for preschoolaged children. It has shown positive effects in reducing tone as well as mobility and gait especially when followed with extensive physiotherapy (Ailon et al., 2015). Side effects include weakness in the associated muscles, in some cases, bladder dysfunction, lordosis, and scoliosis (Ailon et al., 2015).

\subsection{Research Objectives}

- To investigate the effects of physiotherapy interventions from previous literature through a narrative review, to identify the most popular and most recent physiotherapy interventions. In addition, to compare it to the Bobath approach that still shows as the most common type of intervention in paediatric physiotherapy management as it is in Kuwait.

- To investigate the effects of the home program in supporting the effectiveness of clinical physiotherapy interventions through a systematic review, to improve the effects of Bobath therapy on the outcomes of children with CP. Therefore, the parameters of home treatment as a supporting method in addition to a follow up method to promote adherence to home treatment will be investigated from previous literature through a systematic review.

- To explore the clinical physiotherapy intervention provided in PMR hospital in Kuwait using the clinical audit. This is to identify and confirm the levels of achieving set goals and if they are related to lack of home treatment application. The audit aims to inform change and suggests improvement with regard to goal setting and achievement, which can then be implemented in future practice.

- To create a pilot clinical trial that includes implementing an educational course for parents to increase awareness of the effect of home treatment and the role of caregivers at home in Kuwait. Implementing a new method of follow up of 
application of home treatment in Kuwait that includes weekly home visits and comparing it to application of home treatment without follow up in Kuwait.

- To create a satisfactory questionnaire that evaluates the level of satisfaction of the parents that have taken part in the pilot clinical trial in order to evaluate the level of integrity and validity of the pilot RCT.

- To create a survey that evaluates the views and opinions of physiotherapists in Kuwait regarding the clinical physiotherapy intervention provided in the hospital in comparison to the best practice that they believe in. To compare the results with the clinical audit outcomes and identify the causes for those results. To identify the factors that affect the opinions of the physiotherapists regarding methods of practice in Kuwait. 


\section{Chapter 2}

\section{Physiotherapy Treatment for Children with Cerebral Palsy: A Narrative Review}

\subsection{Introduction}

It could be argued that a gap between practice and evidence-based research had resulted in the lack of identified best practice offered for children with CP (Graham et al., 2009). It is therefore essential to further explore the literature to better understand and identify the most recent evidence supporting the available and new interventions. When providing physiotherapy treatment, the families expect the best effective interventions and the health systems depend upon cost-effective services. In the last decade, the CP evidence base has rapidly expanded by providing clinicians and families with the possibilities of newer, safer and more effective interventions (Novak et al., 2013). In the last 10 years, the ability of clinicians to better understand $\mathrm{CP}$ was redefined as intervention options by adopting the World Health Organization's International Classification of Functioning, Disability and Health (Novak et al., 2013). The focus of treatment was, therefore, shifted from addressing physical impairments by promoting functional problems and focus on maximising the children's environment, the independence in daily activities, and community participation. Therefore, clinicians seek to choose interventions guided by what helps families achieve their goals by applying recommended goal based approaches (Law et al., 1997).

Bobath is one of the most common treatments for children with cerebral palsy. However, since it has been introduced by Berta and Karel Bobath for more than 60 years ago (Law et al., 1997), various studies have been evaluating its treatment effects throughout its evolvement over the years (Butler \& Darrah, 2001). As detailed in chapter 1, Bobath is a problem-solving approach to the assessment and treatment of individuals with disturbance of function, movement, and postural control due to a lesion in the central nervous system (Graham et al., 2009).

In the field of physiotherapeutic treatment of children with $\mathrm{CP}$, the literature reflects a range of variation in the application of the approach in addition to confusion regarding the current principles of NDT after evolving. Controversy has risen regarding the principles of 
NDT and whether it is still influenced by the reflex and hierarchical models (Butler \& Darrah, 2001).

Current models do not focus exclusively on the neural explanation of motor performance (Damiano, 2009). Other aspects such as psychological components and environmental context are now part of the model of motor control and the Central Nervous System (CNS) is only one system among many influencing the motor behaviour (Damiano, 2009). The difficulty in assessing the effectiveness of this motor therapy approach or any other therapy approach is that these approaches cannot be delivered in a standardised manner (Antilla et al, 2008). No specific dosage that is administered under constant and specific invariable procedures or maintenance of a specific duration of treatment or intensity could be possible; however, other parameters that depend on therapist skills in addition to specific aims which depend on the patient and patient families vary accordingly (Raine, 2007). The effectiveness of NDT in the literature also depends on the skill level of the therapists providing it (DeGangi \& Royeen, 1994) which is seldom clear; in addition to that, as NDT's structure has changed over time, it is now rarely being delivered without being combined with another therapy technique or medical intervention (Law et al., 1997). Moreover, other factors such as the sample sizes are usually small with a wide range of baseline differences between participants that are also affected by the process of growth and maturation. Furthermore, the choice of outcome measures and measurement tools could have an influence over reported outcomes (research and treatment) and therefore, could affect validating the results and the reliability of the study.

\subsection{Aim}

The aim of this literature review was to investigate the evidence by supporting the different treatment approaches for children with cerebral palsy and furthermore, evaluate the efficacy of the treatment approaches in improving the functional level of children with cerebral palsy. 


\subsection{Methodology}

\subsubsection{Search Strategy}

The literature was searched electronically for studies that have identified home treatment in relation to physiotherapy for children with $\mathrm{CP}$. The literature was searched using electronic databases that included Web of Science, Scopus, CINAHL, PubMed, and The Cochrane Library for articles that included human studies written in English over a 10-year period (i.e. between 2003 and 2014). To assist with the initial scope that determines the extent of the available literature and to test the appropriateness of selected keywords, a search using the Swansea University's iFind research database search tool was completed. Very limited hits were found to be related to Bobath treatment and therefore, the period was extended back to (1989) in order to include a larger number of hits for the Bobath articles only. The following keywords were selected, and combined in various combinations, in order to perform a systematic search of the literature to assist with the identification of papers to be selected as part of this narrative review. Studies were limited to human children, which are related to English language publications that were available in full text. Studies that were not available in full text were not included.

- Cerebral palsy

- $\mathrm{CP}$

- Children

- Physiotherapy/Physical Therapy

- Rehabilitation

- Neurorehabilitation

- Treatment

- Management

- Motor Movement

- Movement Disorder

- Bobath

- Neurodevelopmental Treatment

- NDT 


\subsubsection{Screening}

The citations and abstracts of the papers yielded by the search were read and appraised to confirm their eligibility for inclusion in this review.

\subsubsection{Eligibility and Inclusion Criteria}

Papers were included in this review if they were published between 1989 and 2014 if the full-text paper was available, and the research was evident, relating to physiotherapy treatment for children with CP. Reference lists of the yielded articles were searched to retrieve some more related articles, which is known as hand searching in order to ensure that the review had been inclusive of all the available related articles to the physiotherapy treatment for children with CP. Articles that satisfied the inclusion criteria were also included in the narrative review. Articles' abstracts were then scanned to decide which studies are relevant and which are not to be included in the review.

\subsubsection{Paper Selection}

Duplicates from different databases were discarded. Forty-two articles were obtained after reading the abstracts. Seven articles were discarded after reading the full content article and finding it not relevant to the subject required. A hand search was further performed through the reference lists including articles and 12 more articles were selected from the most articles cited with 100 citations and above and added to the reviewed articles. Using the number of citation as a filter for paper selection, the publications included could be considered to have had an impact in relation to the high-level of knowledge that article had contributed to the research, by showing that it was cited by the research community. The number of articles that had been included to be reviewed was a total of 47 articles.

The literature gathered was divided and reviewed according to the type of intervention provided for the children with $\mathrm{CP}$. 


\subsection{Discussion}

The purpose of this narrative review is to explore the existing literature to identify the various types of physiotherapy treatment approaches that are available for children with $\mathrm{CP}$, in order to manage their functional outcomes (as described in Chapter 1); to examine the application of various research methodologies that will assist the research design process for this research (see Chapter five) and to determine how recent the approaches have been used and explored by the literature as part of identifying the limitations in our current knowledge about the effectiveness of CP treatments.

\subsubsection{Child Focused Therapy and Context Focused Therapy}

Functional therapy, as a form of child-focused therapy, is derived from the dynamic system theory which indicates that motor behaviours are organised around functional tasks and goals (Barber, 2008). They are considered a problem that is solved through spontaneous interactions of variables derived from the child's abilities, the task demands, in addition to the environmental influences (Darrah et al., 2011). The child's abilities and characteristics in this condition include the child's physical impairments as well as the other characteristics such as motivation and cognition. As for the environmental considerations, they consist of the physical accessibility, the assistive abilities in addition to the attitude of the person in the environment of the task. The dynamic theory also stresses the importance of encouraging new motor behaviours. As a child experiences a motor task, encouragement should be offered to develop a new motor skill, which is known as a transition for new motor skills and abilities (Darrah et al., 2011). The best indicator of the development of motor transition is due to parental involvement who, therefore, must be incorporated in goal setting. The main characteristic that makes self-treatment or context therapy more applicable and valid is that it focuses on changing the environment and modifying the task to be achievable by the patient rather than changing the patient's impairments to achieve the tasks as NDT or Bobath.

Ahl et al., (2005) assessed the effectiveness of functional treatment in children with cerebral palsy. Goals were set with the participation of parents, teachers and the children themselves when possible. This was to ensure that the goals were actual functional needs that are required for daily living. Personal functional goals may induce more commitment from both parents and patients when the goals are clearly related to their everyday needs rather than movements or techniques that the parent or child might not feel much related to what 
they need. The outcomes were assessed by either Gross Motor Function Measure (GMFM) or by the Paediatric Evaluation of Disability Inventory (PEDI).

The intervention consisted of a four-day educational course for parents and teachers. The course included a theoretical part that clarified and demonstrated the nature of $\mathrm{CP}$ in addition to the aetiology and normal and abnormal development of the children. It also demonstrated motivation and socio-psychological aspects that were considered essential for therapy. A single three-hour training session per week was given for the children and their parents and teachers in the rehabilitation centre.

The intensity of treatment was set by caregivers themselves and training were documented in a diary with an agreement to be applied at least five times a week. At the end of the five-month intervention period, $77 \%$ of the goals set were achieved. Setting goals played an important role by involving the parents, caregivers and children to understand the purpose of the training and activities in addition to inducing more commitment to the application of the treatment. This study indicated a successful approach of self-treatment of children with cerebral palsy in familiar environments of homes and schools. Akbari et al., (2009) reported that functional treatment was also effective when applied by therapists. This, in confirmation with Ahl et al (2005), indicates that functional therapy is effective whether applied by parents or therapists.

Law et al (2011) had shown the positive effects that child-focused therapy had maintained results after six months and nine months follow up, which indicates the long-term effects that are gained from this approach. Franki et al (2014) had also agreed on the positive effects of functional therapy as long as it was individually designed for each child which had clearly shown stronger effects than general functional therapy.

Context focused therapy is an approach that focuses on changing the environment, which modifies it to better serve the motor needs and abilities of a child with CP rather than trying to change the child's disability. It was investigated by Darrah et al (2011) and Law et al (2011). Both studies have found context therapy to have positive effects in making the children more independent. However, the intervention failed to maintain results after six months follow up. Additionally, this intervention had raised concerns with some parents that did not appreciate this type, as it did not involve any hands-on treatment. 


\subsubsection{Stretching}

Akbayrak et al. (2005) investigated the effect of positioning in an antispastic position for the ankle joint of children with spastic diplegia. Results of before and after measurement of range of motion and spasticity after a 20-minute intervention was effective. Damiano (2009), however, found it to be less effective. Damiano (2009) claimed that there was limited evidence supporting long-lasting effect of stretching in the literature, despite it being a very common intervention for children with CP. According to the study, in reality, it is either underdosed or ineffective due to immobility or unwanted muscle activation due to spasticity. However, stretching could be more beneficial if applied in higher intensities by cast or orthosis or even applied actively via dynamic stretching in functional activities to prove more effective.

However, other studies (Franki et al, 2012, Curtis et al, 2014) had found positive effects of stretching as long as they are applied passively or dynamically for longer than 30 minutes.

\subsubsection{Bimanual Therapy}

Bimanual therapy differs from conventional therapy in two ways. Firstly, the intensity is far greater which provides the opportunity for motor learning through practice. Secondly, during treatment, the child is encouraged to use their involved hand as any other typically developing child uses their non-dominant hand and focus is referred to coordination of hand and arm at the end of the movement (Charles \& Gordon, 2006).

Brandao et al. (2014) demonstrated significant changes after an intensive two-day treatment course of bimanual therapy. It should be mentioned that the intervention of bimanual training was not delivered by a qualified physiotherapist. This indicates that this intervention does not necessarily require qualification before applying, which indicates that parents and caregivers may provide the intervention at home as long as they receive some training. The intervention consisted of six hours per day which the authors have indicated and may have been the major contributor to these findings. However, Brandao et al. (2014) suggested that a lower dosage of intervention may not reveal the same results for this very intervention. In addition, it is not certain that immediate results after an intensive two-day course would indicate a significant change in a child level of activity especially when 
intervention does not include functional therapy and is not delivered by a qualified physiotherapist. In addition, Aartz et al (2010) had investigated the effects of bimanual therapy using an intensive approach of 9 hours a week of bimanual therapy following another intervention. The results showed no significant changes in the research group, the total duration of the intervention had extended to a total of 8 weeks.

\subsubsection{Constraint-Induced Movement Therapy}

Constraint-Induced Movement Therapy (CIMT) is a treatment specifically created for upper limb function improvement in children with hemiplegia. The CIMT program includes restraining the use of the non-affected limb of approximately $90 \%$ of the waking hours, in addition to training the affected limb up to 7 days a week for approximately two to three weeks. The technique had shown increased levels of functional abilities of the affected upper limb in addition to a concomitant cortical reorganisation. Restraining of the limb may be seen in different forms depending on the tools used. Activity programs include selected tasks that difficultly increased, depending on success, to achieve the given task which is known as the shaping process (Barber, 2008).

\subsubsection{CIMT for Children}

According to Charles and Gordon (2006), CIMT has several conceptual problems that should be considered when being applied to children with cerebral palsy. The intervention has been developed to overcome the non-use in adult hemiplegic patients. However, children with cerebral palsy must overcome developmental non-use of their affected limb as they might have never learnt to effectively use their involved limbs. This requires the approach to be modified in order to have a developmental focus. Another concern is the level in which the approach is considered invasive, constraining a child with cast or other modalities is not quite conservative and could be considered invasive by many therapists and caregivers. As for bimanual impairments, they are seen much more in children with hemiplegic CP beyond unilateral impairments, as stated by Charles and Gordon (2006). The associated brain damage of hemiplegia usually involves the supplementary motor area and the parietal lobe. Bimanual intervention retains intensive practice, which is the key successful element of CIMT and targets known deficits in bimanual coordination in order to utilize the principles of motor 
learning and finally utilises the principles of neuroplasticity. This supports the evidencebased medicine dictation that is being designed on knowledge of how the nervous system develops and functions as well as the need of solid understanding of the neural bases of the specific motor impairments.

As for the age of children suitable for CIMT, Coker et al. (2009) have assessed the feasibility and effects of CIMT on younger children. The study had assessed the intervention on a single case of hemiplegic CP of 9 months of age. The intervention had included a modified CIMT (mCIMT) approach. Significant changes were seen after one month of intervention in both the GMFM and The Peabody Developmental scale. It must be mentioned that after the conventional therapy period, the child did not show any changes in progress but had rather only maintained the results attained from the mCIMT intervention. The results of this study had shown the feasibility and effectiveness of the mCIMT approach in children less than 1 year of age. In addition, after six months' follow-up, the child had also shown maintenance and a slight improvement in the attained levels of function due to the treatment received, which indicate the continuous long-term maintenance of results of the mCIMT, which is in consistency with Charles and Gordon (2006). Klingels et al. (2013) had also agreed on the same findings and indicated that results may be improved if more than one intervention was used at the same time. Another finding were that younger children have shown a higher level of improvement compared to older children in both groups. Also, children with lower abilities and functional level have shown higher levels of change when compared to children with a higher level of functional abilities at baseline.

\subsubsection{Parameters and Effects of CIMT}

In their study to assess the effect of intensive CIMT, Deluca et al. (2006) had investigated the intervention on 18 children between the ages of 7 months and 8 years of age. Results indicated a significant change in the treatment group compared to the control group that showed no significant difference before and after the three-week period. This was maintained even after the three weeks follow up of results. Although such intervention has proved effective in changing the functional level, it could be argued that a fibreglass cast applied 24 hours a day on the unaffected side of a child with unilateral cerebral palsy may be considered to be slightly harsh. As mentioned, some children have suffered from skin abrasions, which were managed by ointments and extra padding before recasting the arm for 
another week. Casting may also cause the children to be frustrated as they are unable to use their unaffected arm. This might affect the participation in the intervention and home activities. Although the objectives and hypothesis of these have been fulfilled methods and procedures would have to be revised for future studies.

In assessing the long-term effects of CIMT, Charles and Gordon (2006) had assessed children with hemiplegic CP after one week, one month, six and 12 months' follow-up, after a treatment course of CIMT. Results of this study had shown significant 12-month retention of results attained from a CIMT intervention course. This had indicated the continuous effect of the treatment on changes in functional levels attained by the children in response to the treatment that had proven a long-term improvement. This period was found to be the longest follow-up in CIMT studies in children with CP. Similar to any long-term study of children with $\mathrm{CP}$, the possibility of development still stands and may have partially had an effect on the results attained, however, the control group in this study had failed to attain and maintain the results found in the intervention group. However, in 2007, Gordon et al. had questioned the effectiveness of CIMT for children with CP, especially that if it was developed with the unlearned non-use of affected limbs in adults. This may not completely apply to children with $\mathrm{CP}$ that may have never learned to use their affected limbs in the first place and furthermore, the invasiveness of the constraint itself on children. Therefore, the need for modification of the approach whenever it is used for children is being suggested.

According to the need of modification of intensity and aggressiveness of CIMT, Smania et al. (2009) have investigated the effect of modified CIMT (mCIMT) in children with hemiparesis in a randomised crossover design trial. Modified CIMT is a modified version of the intervention that promotes fewer hours of therapy a day, which should be about $90 \%$ of hours per day in CIMT in addition to the reduction of the intensity of the constraining method. In this study, the constraint method consisted of a cotton mitten that prevented the child from grasping with their uninvolved hand. The results have shown a significant difference in the hand function test in favour of the mCIMT group, the results indicate the effect of intervention with the conventional therapy that the children in the group were receiving and does not favour the mCIMT by itself. Other results indicated the maintenance of the effects after follow up period of 4 weeks. Issues and limitations of the CIMT were raised in this study, noting that the issues related to the use of CIMT were the frustration of children due to the inability to use the preferred limb induced reversed effects and resulted in children not wanting to focus on the free affected extremity. The application of such 
intervention was indicated as a chance of affecting self-esteem and the psychological refusal of the treatment intervention overall. Distress was also noted in parents when applying the intervention that revealed to them how disabled their child was. This is a psychological aspect that other studies have failed to identify.

Myrhaug et al 2014 had assessed the effect of treatment approaches that included CIMT and other interventions in a systematic review. The review had found CIMT to be effective with a positive change in controlled before and after studies as well as when compared to other interventions. However, these results depended highly on the intensity of the treatment given as shown in some studies (Dong et al., 2013; Eliasson et al., 2011; Sakzewski et al., 2014), in the review that CIMT has shown equal results to conventional therapies when delivered in the same intensity and frequency of sessions. This was raised in other studies assessed by this review (Eliasson et al., 2011; Sakzewski et al., 2014), indicating the lack of knowledge of the optimum intensities and other parameters of treatment interventions available for cerebral palsy. Another parameter of effectiveness is the age of the child which is in agreement with Klingels et al (2013). Young children would develop more change in motor abilities compared to older peers.

\subsubsection{Strengthening}

According to Damiano (2014), the strengthening approach may be one of the most important and strongest evidence-based approaches for the improvement of gait patterns of cerebral palsy. Even though some studies (Scianni et al, 2009) found that strengthening was ineffective for cerebral palsy, Damiano (2014) argued that Sicanni et al (2009) findings were due to the inefficient principle of loading muscles to induce strength changes. Therefore, Scianni's findings were likely due to inaccuracies in the application of the intervention. On the other hand, other studies (Damiano et al, 2002, Steele et al, 2012) that promoted strengthening for more than one case of crouched gait of cerebral palsy after individually considering the type of strengthening required according to the level of spasticity in each case, had shown successful positive changes in the gait patterns. These findings would support not only the strengthening approach but would also shed light on the importance of the need for individualising the treatment approaches for each individual case of cerebral palsy. This was also previously indicated by Blundell et al (2003) confirming in their study the positive effects of strengthening. Objective measures have shown immediate positive 
improvements in addition to maintaining the results after 8 weeks follow up, which indicate the continuous effect on the change in muscle strength. Similar results were also found by Dodd et al. (2004), that had included a population of older children in a home-based strengthening approach, only self-perception and positive emotional changes towards selfconfidence were found to show significant changes and improvement.

Franki et al. (2012), in agreement with Damiano's results in 2014, had considered strengthening with its various ranges to be effective in most of the studies evaluated in the review. The negative effect seen was referred according to Franki et al. (2012) to shorten the duration of the intervention period in addition to the training intensity. Strengthening is an approach that requires periods longer than other interventions to provide significant results, given the nature of the muscle response. Another factor that might have hindered the results in these studies was the outcome measures of the results. These studies have recruited muscle volume and energy expenditure as outcome measures. Another study that did not show significant results had investigated the approach using a home setting and self-treatment approach that might not have been monitored closely. Other studies that used isotonic strengthening had shown insignificant results that could be referred to the nature of spasticity of cerebral palsy, which might not respond properly to such types of training that highly provokes spasticity. Strong results found in strengthening interventions by Franki et al (2012) were in functional techniques performed in the home environment upon agreed exercises by both therapists and parents. A decrease in the level of performance was seen in almost all the studies assessed by the review after a period of no training. Similarly, Demuth et al (2012) had also found no significant effects of strengthening through cycling for children with $\mathrm{CP}$ between 7 and 18. The results have failed to show any significant effects in favour of the cycling group that were recruited for an hour of cycling divided into strengthening and cardiorespiratory phases.

\subsubsection{Bobath}

In their review of effective interventions, Antilla and colleagues (2008) had found functional therapy to be more effective than NDT in both GMFM results and PEDI, which they have indicated as a limited evidence. Other studies had indicated limited evidence in supporting higher benefits of intensive NDT compared to regular intensities while other studies had shown no difference in the effect of NDT when delivered in different intensities. 
According to Papavasiliou (2009), Bobath is a method that is used to reduce abnormal patterns of movement and posture by normalising the tone in the muscles, inhibit primitive and abnormal reflexes to promote normal patterns and movement in children with cerebral palsy. The concept has evolved over the last 50 years and now embraces neuroplasticity as the main principle of neurological recovery.

Desloovere et al (2012) assessed the NDT approach in children with cerebral palsy after botulinum toxin A. In this study, the NDT group showed significantly higher results than the conventional control group, however, many factors could have led to such findings. The study reported that various differences including age, level of GMFCS in addition to the choice of treatment techniques used, were found in both groups. The study used interchangeable treatment methods in both groups, therefore, the treatment approaches were not purely NDT or conventional. Furthermore, the study also mentioned that some therapists in both groups did not perform inhibition techniques, which is the key principle of NDT. Thus, showing that the interventions were not purely delivered and therefore, no comparison can be made between the two groups in terms of treatment type results.

According to Larsson et al. (2012), providing a fully functional approach intervention in a familiar environment such as the home may result in daily activities objectives to be achieved. However, it may not necessarily mean that changes and prognosis have been reached in a child. Each child capabilities physically and psychologically are unique and in many cases, the physical encouragement is necessary to achieve full capabilities of a child that may not achieve independently. This may result in a child settling for what they encounter at the time, depending on the modification of the home environment being applied to their current physical states. Many caregivers that have experience previous experiences in hands-on and disability reversing focused interventions may not approve of this type of intervention compared to hands-on therapies.

\subsubsection{The effect of the Bobath Approach on Functional Level of Children CP}

DeGangi and Royeen (1994) implemented a survey that targeted the NDT association members in the USA by investigating the parameters by which NDT therapists are applying the treatment. Respondents focused on the application of specific NDT techniques in more than $75 \%$ of the treatment sessions while the rest of the sessions were used to observe the patients' movement in order to evaluate the level of change in response to treatment. 
Therefore, they provide the most suitable updates to treatment provided. In addition, more than $86 \%$ had provided families with home treatment according to the patient's needs. When assessing the attitudes of therapists regarding the intervention, more than $90 \%$ agreed that the Bobath approach is the treatment to choose for the treatment of patients with neuromuscular dysfunction, even though the original concepts set by the Bobath approach must be revised. However, a great number of responses thought that the Bobath approach is best provided when coupled with other treatment techniques. In addition to the importance of including the family in the treatment program which was agreed on in more than $98 \%$ of responses.

When asked if NDT training courses were important, more than $67 \%$ thought that in order to use NDT, a therapist must attend the training courses in order to apply the intervention effects. Additionally, $83 \%$ of respondents thought that further research is needed to confirm the effectiveness of NDT.

In their discussion, DeGangi and Royeen (1994) referred that NDT had changed over time since it had been presented, given that many NDT therapist is applying the treatment in combination with other interventions. This suggests that the NDT on its own may not be as effective in achieving functional goals, which may justify the need expressed by the respondents for further research in the effectiveness of the approach (Degangi \& Royeen, 1994). In addition, the originators of Bobath intended that NDT be applied differently, depending upon which discipline of therapy (that is, physiotherapy, occupational therapy, or speech therapy) was delivering NDT, so that the intervention would be delivered in accordance with their respective discipline, specific goals and methods of application, yet the survey found that respondents had falsely believed that all the disciplines were providing the same type of NDT therapy. This was thought to be a result of the NDT training courses that are provided similarly for the three disciplines. However, the differences in the application of the approach were believed to be referred to the differences in basic knowledge between disciplines. As for best practice, two perspectives were identified by the survey that would identify best practice which included the involvement of family within the treatment goals and treatment application in both the treatment environment and at home, as well as the effectiveness of improvement of functional level.

The survey also revealed an important conflict between beliefs of the intervention and actual practice. Respondents mostly agreed upon NDT being a total management program, which should consider the functioning of the patient in different settings. However, only 53\% 
had agreed that carrying over the treatment into the home environment and training the parents in such an environment would be a critical aspect of treatment. As for intensities of treatment, the study failed to emphasise the details of this aspect. No justification was provided for the chosen intensities of treatment sessions per week.

Knox and Evans (2002) assessed the effectiveness of Bobath on a functional level. The children showed significant improvement in the scores of goals focused on GMFM and not in the total scores of GMFM. It was also noticed that children with higher levels on the GMFCS and younger children have shown higher significance than other children in the group who were older or with lower levels of GMFCS (IV, V), who did not show any significant changes. PEDI scores have also shown significant improvements in many categories.

Embrey and colleagues (1990) had assessed the effect of NDT on a single case of a 2year-old girl with hypotonic diplegia with $2+$ deep tendon reflexes in upper and lower extremities. The objective of the study was to evaluate the effect of NDT on the hyperflexion of the knee joint during gait when applied with the use of the orthosis. Measurements were taken every week. Results showed no significant change in the knee angle during gait. Although some changes were seen after the week of treatment and after the week of treatment with orthosis; however, none of the changes was reported to be statistically significant. Intervention, in this case, was given for a very limited period of time, in addition to that, a whole week of no treatment followed each phase of the intervention with no logical explanation. The intention perhaps was to differentiate the effects of the NDT alone from the effect of the combined NDT and orthosis approach. However, the short period of intervention per week is considered very limited. The outcome measures of the gait may have affected the findings in this study as it could be argued that a more developed gait lab that would assess the gait in a three-dimensional aspect would have shown different results. Reliability of the measures could be questioned as no valid measures have been taken.

Similarly, Lilly and Powell's study in 1990 also used a single subject design study that was repeated on two patients with spastic diplegia. The results also did not show any significance in either of the patients. The authors have referred these findings to a lack of interrater reliability due to choosing functional daily activities as the outcome measure. It could be argued that the methodology of this study had shown many factors that could have affected the results, including the outcome measurement that was of no standardisation what 
so ever. It is possible to choose an outcome measurement that measures the functional activity, which was what the authors have intended in addition to being standardised, valid and reliable at the same time similar to the GMFM. Another factor that might have affected the results would be the blinding lack of the assessor which highly affects the reliability of the effects, given that the same therapist had also provided treatment to the decreased frequency of the intervention sessions. Results may show higher levels of significance if the frequency of treatment was slightly increased as well as using an outcome measurement with higher reliability and validity.

No significant results were either found in favour of NDT in the study of Kluzik et al., in 1990. The procedure was then to receive a session of NDT of 35 minutes, followed immediately by another reaching assessment to evaluate the effect of the treatment session. The treatment was reported to focus on reducing spasticity during movement and facilitate appropriate weight shifting and postural reactions. Measurements were taken using motion analysis equipment. Only one subject had shown a significant change in movement time measurements. Although the other 4 children have shown some changes, none were reported to be significant.

In contrast, positive results supporting NDT were found by Adams and his colleagues in 2000 when the effect of NDT on gait was evaluated. The intervention has been individualised for each child's case and abilities. Significant changes were seen in stride and step lengths in addition to velocity and foot angles after the six weeks of intervention. The category that showed the most benefit from the intervention was surprisingly for the 18 children with diplegia compared with the 11 children with hemiplegia. This indicates the effect of NDT in improving the gait of children with diplegia. Children with hemiplegic types of $\mathrm{CP}$ are usually the most independent ambulant type of $\mathrm{CP}$ in addition to gaining most benefits from gait training.

The results of this study reported beneficial effects upon motor function, even for other children with other types of CP that include tetraplegia and athetoid CP. Results have shown positive changes in children with all types of CP in this study in all the aspects assessed, however, significance was seen only in part of the measurements. It could be assumed that different parameters of intensity and duration of intervention might even have higher changes that might reach significance for all aspects assessed and for all types of CP. 
A trial by Chochowska et al., in 2008 included 45 children with cerebral palsy for a duration of three years, where the children were assessed every six months to investigate the factors that affect the efficacy of Bobath in improving functional level. The study had discussed the type of CP and the severity of the disability to be an important factor with a major effect of the changes permitted by Bobath, in addition to the time of diagnosis of the lesion which is also highly affected by the severity of the lesion itself. As in many cases according to the authors' argument, the cases of CP that are severely affected are the earliest in detection compared to the mild cases. This is not in favour of the cases with mild symptoms of delay leading to intervention taking place later in the child's life. This was shown in the study to have high effects on the level of change induced by Bobath and may be generalised to hinder the effectiveness of any intervention that is introduced significantly later in the child's life. The study had also indicated that the children with $\mathrm{CP}$ that are cognitively affected by their lesion show a lower level of development in response to the intervention compared to other children that have a cognitive delay in addition to their motor problems. It should be mentioned that the study depended on their assessment of measurement tools, which were not found common in the literature. In addition, the authors have not supported their use of such tools with evidence validity and reliability evidence.

In agreement with Papavasiliou (2009) and Damiano (2009), Bobath therapy has undergone research in the past two decades and had failed to prove neither effective nor ineffective. The approach could not prove to be superior to any other intervention. Furthermore, in other studies of long-term effect, the approach proved ineffective.

\subsubsection{Comparison between the Bobath Approach and Other Interventions}

In the belief that NDT is better applied to associated interventions, Desloovere et al (2012) investigated the effect of Bobath when applied with botox- a treatment for children with CP. Results have indicated higher levels of change on the GAS scores after NDT intervention compared to the conventional therapy group. Intervention in both groups had also included casting in addition to Botox. An important finding in this study was that the differences between the physiotherapy programs applied in the conventional group and the NDT group were found to be very limited. The NDT therapists were found to spend less time on tone reduction and mobilisation than therapists in the conventional therapy group while these are considered core skills of the original Bobath approach. This also agrees with 
DeGangi and Royeen (1994) that Bobath is changing and is not only focusing on reducing tone and facilitating movement but also is trying to translate additional patterns of obtained activity by handling into practice the required skills, especially that practice will not teach a child to perform skills that are not previously experienced. It was also found that even though children of unilateral CP that have a higher functional level of I or II on the GMFCS scale have shown a higher average of the change in GAS scores in response to treatment in both groups, the children with bilateral $\mathrm{CP}$ showed more significant changes in response to treatment of NDT. These findings indicate that children with a functional level of I or II on the GMFCS may be less sensitive to treatment approaches compared to the children with more severe types of CP that have a lower functional level and had shown a higher level of response to the NDT group. This agrees with Chochowska et al., (2008) that the type and severity of CP has a high influence on the effectiveness of NDT on functional improvement. However, Chocowska et al., (2008) had referred this conclusion to more severe cases of CP such as quadriplegic cases. Therefore, it could be concluded from both studies that very mild and severe cases of CP both have limited response to NDT treatment.

Upon searching the literature for their review, Butler and Darrah, back in 2001, had included only studies that have exclusively assessed NDT or NDT-based therapy, in addition to NDT combined with sensorimotor interventions. Results have indicated with respect to change in the neural based motoric responses of the CNS that up to thirty measures have evaluated such changes known as qualitative movements or physiological motor function, reflex activity, weight shift, postural alignment, trunk rotation, associated reactions and several aspects of Upper limb movement and gait. Eight studies had found that better motoric responses were associated with NDT. However, this evidence of improvement of physiological motor function and qualitative movement was not consistent. In contrast, other findings have shown that tone, spasticity and reflex responses were either not changed or change was further observed within the control group. Other studies that assessed more intensive intervention of NDT showed no better results and were studies of weak levels of evidence. Evidence found in the category of NDT slowing or preventing deformity and contractures, results were found to be in favour of NDT in more than one study that confirms the advantage of NDT in dynamic measures of range of motion after the intervention. In contrast, no effect was seen of NDT in static measurements of range of motion which were presented in studies with stronger levels of evidence that assessed the effect of NDT in intervention durations of up to 12 months. 
Bar-haim et al (2006) indicated that there were no significant changes after one month of intervention in the NDT group compared to the other group using the ADELI suit. Although intensive treatment was delivered in this study, the short duration of the intervention might have hindered any effect of the intensity. The age range of children may have also played a role in limiting the effect on their abilities for changing functional level. Similarly, no significant findings were detected in favour of the NDT group in Dalvand et al (2009), where NDT was compared to conductive education and parents' education as an independent type of intervention. The results showed the significant result in favour of the CE group in 11 out of 16 items in an ADL outcome measurement. The findings of the study were referred to the effect of group treatment of the CE group and that encouragement was made by the peers of that group by encouraging them to achieve goals. Socialisation and group work was also appreciated as a motive for more achievements in that group of intervention. 


\subsection{Conclusion}

Studies in paediatric physiotherapy remain inconclusive and would be difficult to effectively reproduce due to the limitations in the literature report containing key elements of methodological and interventional descriptions, such as the sample size calculations, randomisation procedures, and concealment of group allocation. This is to ensure blinding of assessment from participants in addition to providing information regarding the delivery of any co-interventions and how the assessor's blindness was evaluated. Moreover, available reviews that had discussed the effects of the treatment available on children for each of the differences seen in individual or groups of children that may serve as a source of information to advance clinical practice while they are not considered enough to provide data that is necessary to change practice and change treatment decisions.

As numerous research studies have been made to assess the effect of the treatment approaches available they provide an important source of information for advancing clinical practice. However, studies have failed to provide the necessary data to change general treatment decisions for physiotherapy treatment of children with CP (Damiano, 2014). The majority of published studies have pointed out the main effects of group differences but with minor attention to individual difference given that cerebral palsy is quite a heterogeneous population varying widely in type, timing, location and extent of brain injury. Therefore, advance should be made in the evidence-based research for management of children with cerebral palsy (Damiano, 2014).

In summary, many of the studies assessing the effectiveness of Bobath had shown inconsistent evidence to support the effect on functional level improvement. Evidence found in favor of NDT or Bobath treatment in the category of slowing or preventing contractures and deformities in addition to improving quality of gait patterns and speed. Results were found to be of the highest efficiency when applying Bobath with other approaches that would help in supporting the child's needs as a whole, and that would provide the child with a higher variety of techniques the would promote higher and more significant changes in functional levels in different environments.

The GMFM, on the other hand, was found to be the most common measure used that measures the child's functional level as a whole rather than measure some functions similar to gait analysis that may not be applicable for all ages of children or all levels of severity of CP. 
A finding from this narrative review suggests that Physiotherapy is best provided for children with CP continuously, which supports the points mentioned in the previous thesis chapters. With this review indicating that Bobath could be recommended as an appropriate physiotherapy treatment approach for $\mathrm{CP}$; however, to ensure the continuation of the observed beneficial effects it is necessary for further investigations to be completed to evaluate another important principle associated with the application of Bobath. On the basis of the results from this narrative review and combined description of the principles of Bobath (as outlined in chapter 1), it could be postulated that a supporting intervention such as a home treatment (exercises recommended by the physiotherapists to be applied at home in specific parameters for the management of children with $\mathrm{CP}$ ) could be effective to ensure the maintenance or to increase the level of outcomes generated during the physiotherapy, which was led by the Bobath treatment sessions to ensure continual treatment at home and in different environments that involves parents and daily activities. Further investigation is done in the following chapter to further identify the evidence supporting home program. 


\section{Chapter 3}

\section{The Effect of Home Treatment in Supporting Functional Development: A Systematic Review of the Literature}

\subsection{Introduction}

As seen in chapter two home treatment may have an important role in supporting physiotherapeutic intervention in promoting and improving functional development in children presenting with CP. However, as the home treatment is applied by parents and caregivers, it is helpful. According to the Bobath approach's main principle, to provide a method of follow up in order to ensure the adherence to the application of home treatment in addition to the quality of the home treatment provided. This was highlighted in chapter two by the studies explored. In addition, from the previous clinical experience of management of children with $\mathrm{CP}$, the follow up was found to be appreciated as a supportive method for parents to provide encouragement and promote adherence to the home treatment.

Motor disability in children with cerebral palsy affects motor functioning and, therefore, this could affect their independence and quality of life (Novac et al., 2013). Children with cerebral palsy receive physiotherapy interventions to improve the quality of their movements almost continuously throughout their lifespan (Law et al., 2011). The aim of therapy is to ensure as much independence to the child as possible. To improve effectiveness and outcomes of treatment studies have assessed supporting treatment approaches with various types of home treatment (Bilde et al., 2011; Katz-Leurer et al., 2009). Home treatment can be used as an alternative to institutional physiotherapy when clinical physiotherapy treatment is not available due to many reasons. It could also be provided to support the intervention and increasing outcome of clinical physiotherapy (Novak et al., 2013). Another reason for providing home treatment would be to ensure continuous treatment when the patient is off the institutional intervention to prevent any deterioration of the treatment outcomes.

However, the home treatment itself is also a wide phrase that may vary widely in the types of activities included (Tang et al., 2011). The decision regarding the most suitable home management will depend on the purpose of the home treatment itself in addition to the level of activity of each child and the area in which the therapist wishes to improve and 
concentrate on. When deciding among the suitable home treatment consideration should be given to the parents' state of cooperation in order not to stress out caregivers as such stress could result in negative outcome on the home programme and institutional treatment as well (Halvarsson et al., 2010). Furthermore, family priorities, needs and participation can be considered a major factor in the decision regarding the type of the home programme given to that child depending on the child's age and motor function level (Chiarello et al, 2010).

In addition, in Kuwait the awareness of the importance of the home treatment role and effects of application is limited among caregivers and in many cases, is overlooked. Therefore, caregivers in Kuwait are rarely committed to home treatment, and an objective evaluation of the effectiveness of home program has not been conducted before in Kuwait.

\subsection{Aim}

The aim of this chapter is to explore the research evidence relating to the effect of applying management for children with $\mathrm{CP}$ at home by parents in promoting maintenance or development of functional level in Kuwait.

\subsection{Objectives}

The objective of this review is to undertake a systematic review to critically appraise the research evidence relating to the effects and intensity of home physiotherapy treatment provided by the parents for children with CP in addition to the types of follow up methods and their effects on promoting adherence and application commitment to be compared to the limitations in application of home treatment in Kuwait. The review was also intended to identify a follow up method to be implemented in Kuwait"

\subsection{Methodology}

In order to identify the relevant literature relating to home treatment for children with CP including adherence to home treatment, the methods of follow up of home treatment, and the effectiveness of the home treatment on a functional level of a number of methods have been employed. 
Electronic searches, hand searches and a quality criterion were employed to perform a comprehensive search of the literature available.

\subsubsection{Search Strategy}

The literature was searched electronically for studies that have identified home treatment in relation to physiotherapy for children with $\mathrm{CP}$. The literature was searched using electronic databases that included Web of Science, Scopus, CINAHL, and Pub Med for articles that included human studies in English between the years of 2003 and 2014. To assist with the initial scoping to determine the extent of the available literature and to test the appropriateness of selected keywords, a search using the Swansea University's iFind research database search tool was completed. The following keywords were selected, and combined in various combinations, in order to perform a systematic search of the literature to assist with the identification of papers to be selected as part of this narrative review.

- Children

- Handling

- Home Handling

- Home Management

- Home Therapy

- Home Treatment

Keywords were combined and used alternatively after being used individually to narrow down the search and limit the number of articles found. Studies were limited to human children related, English language publications that were available in full text. Studies that were not available in full text were not included.

\subsubsection{Screening}

The citations and abstracts of the papers yielded by the search were read and appraised to confirm their eligibility for inclusion in this review. 


\subsubsection{Inclusion criteria}

Papers were included in this review if they were published between 2003 and 2014 if the full-text paper was available, and the research was evidence relating to the effects of home physiotherapy treatment for children with CP. Reference lists of the yielded articles were searched to retrieve some more related articles, which are known as hand searching. Articles that satisfied the inclusion criteria were also included in the systematic review. Articles' abstracts were then scanned to decide which studies are relevant and which are not to be included in the review.

\subsubsection{Eligibility and Inclusion}

\subsubsection{Quality Criterion}

The CASP critical appraisal tool was used for the appraisal of the papers of this review. CASP represents Critical Appraisal Skills Programs and provides a practical framework to identify appraisal information, to ensure the best evidence is used in health decisions. This is done by following a set of critical appraisal tools that facilitate a systematic approach to examine the researches validity and reliability to consider its relevance to a specific context that would inform healthcare decisions (Critical Appraisal Skills Program, 2010).

The 'CASP' checklist (Appendix 3) consists of 12 conditions, with a possible total score of 10 if all 12 conditions are fulfilled. Part A (one to six) assesses the internal validity of a study and is a score out of 8 . Then, the results are assessed by part B (seven-nine) and are scored out of one. Additionally, part C (10) refers to the application of the findings to the local population and is scored out of one. Points are only awarded if a criterion is clearly satisfied and reported. Criteria 11 and 12 refers to the implications of the findings for practice, and these items are not used to calculate the CASP score but are included to consider the relevance of the findings within a healthcare context. Papers were included if they yielded a combined score of seven or above ensuring quality appraised studies were included in the review. 


\subsection{Results}

\subsubsection{Search History}

The tables below display the search history and the number of papers yielded for the different combination of words from IFind, Web of Science, Scopus, CINAHL, Pub Med databases, between the years 2003 and 2014.

\subsubsection{Eligibility \& Inclusion}

The papers included in the review are summarised in the table below with the CASP score.

Table 4.1. Showing the CASP scores for the articles included

\begin{tabular}{|l|l|l|}
\hline Author(s) & Title & Score \\
\hline Basaran et al (2014) & $\begin{array}{l}\text { Adherence to Home Exercise Program among } \\
\text { Caregivers of Children with Cerebral Palsy }\end{array}$ & $7 / 10$ \\
\hline Bilde et al (2011) & $\begin{array}{l}\text { Individualised, Home Based Interactive Training } \\
\text { of Cerebral Palsy Children Delivered Through } \\
\text { the Internet }\end{array}$ & $9 / 10$ \\
\hline Bryanton et al (2006) & $\begin{array}{l}\text { Feasibility, Motivation, and Selective Control: } \\
\text { Virtual Reality Compared to Conventional Home } \\
\text { Exercise in Children with Cerebral Palsy }\end{array}$ & $9 / 10$ \\
\hline Chen et al (2014) & $\begin{array}{l}\text { Home Based Tele Assisted Robotic } \\
\text { Rehabilitation of Joint Impairments in Children } \\
\text { with Cerebral Palsy }\end{array}$ & $8 / 10$ \\
\hline Halvarsson et al, & $\begin{array}{l}\text { From Authority to Coach Parents' Experiences } \\
\text { Health 14 Months After an In-Home Virtual } \\
\text { Reality Video Game Hand Telerehabilitation } \\
\text { Intervention in an Adolescent with Hemiplegic } \\
\text { Cerebral Palsy }\end{array}$ & $9 / 10$ \\
\hline Golomb et al (2011) & $8 / 10$ \\
\hline
\end{tabular}




\begin{tabular}{|c|c|c|}
\hline (2010) & $\begin{array}{l}\text { of Stretching as a Home Programme for Children } \\
\text { with Cerebral Palsy }\end{array}$ & \\
\hline Harbourne et al (2010) & $\begin{array}{l}\text { A Comparison of Interventions for Children to } \\
\text { Improve Sitting Postural Control: A Clinical } \\
\text { Trial }\end{array}$ & $8 / 10$ \\
\hline $\begin{array}{l}\text { Katz-Leurer et al } \\
\text { (2009) }\end{array}$ & $\begin{array}{l}\text { The Effects of a Home Based Task-Oriented } \\
\text { Exercise Programme on Motor and Balance } \\
\text { Performance in Children with Spastic Cerebral } \\
\text { Palsy and Severe Traumatic Brain Injury. }\end{array}$ & $7 / 10$ \\
\hline $\begin{array}{l}\text { Novak and Cusick } \\
\text { (2006) }\end{array}$ & $\begin{array}{l}\text { Home Programmes in Paediatric Occupational } \\
\text { Therapy for Children with Cerebral Palsy: } \\
\text { Where to Start }\end{array}$ & $10 / 10$ \\
\hline Novak et al (2007) & $\begin{array}{l}\text { A pilot study on the Impact of Occupational } \\
\text { Therapy Home Programming for Young } \\
\text { Children with Cerebral Palsy }\end{array}$ & $10 / 10$ \\
\hline Piggot et al (2002) & $\begin{array}{l}\text { Participation in Home Therapy Programs for } \\
\text { Children with Cerebral Palsy: A Compelling } \\
\text { Challenge }\end{array}$ & $9 / 10$ \\
\hline Taylor et al (2004) & $\begin{array}{l}\text { Factors Influencing Adherence to a Home Based } \\
\text { Strength Training Programme for Young People } \\
\text { with Cerebral Palsy }\end{array}$ & $9 / 10$ \\
\hline Wang et al (2013) & $\begin{array}{l}\text { A Home Based Program Using Patterned } \\
\text { Sensory Enhancement Improves Resistance } \\
\text { Exercise Effects for Children with Cerebral } \\
\text { Palsy: A Randomised Control Trial }\end{array}$ & $8 / 10$ \\
\hline Weindling et al (2007) & $\begin{array}{l}\text { Additional Therapy for Young Children with } \\
\text { Spastic Cerebral Palsy: A Randomised Control } \\
\text { Trial }\end{array}$ & $7 / 10$ \\
\hline
\end{tabular}




\subsubsection{Inclusion}

The Preferred Reporting Items for Systematic Reviews and Meta-Analysis (PRISMA) flow diagram provides a summary of the information necessary to follow the different phases of this systematic review (Fig 3.1). It displays the number of papers identified that have been included and excluded from the review (PRISMA, 2009). Ninety-three articles were originally retrieved and were deducted to 33 after applying the stages of PRISMA using the flow diagram. Duplicates were removed, and after the records have been screened, many were excluded either for not having the full text available or for not being quite related to the topic of home treatment for children with CP. The 33 articles were reduced to 14 after being assessed and reviewed using the CASP critical appraisal tool (as mentioned previously). 


\section{PRISMA 2009 Flow Diagram}

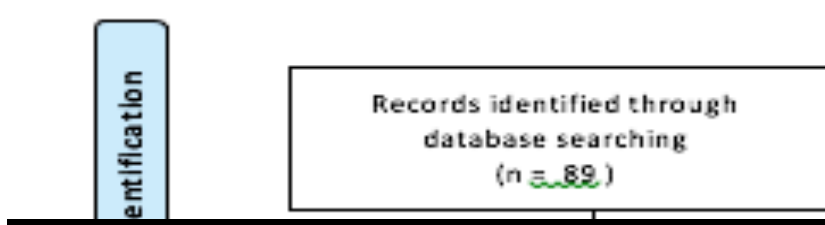

Additional records identified through ather sources in $=4$,

$$
\text { in }=14 \text { in }
$$




\subsection{Discussion}

This systematic review was aimed to identify the evidence supporting the effect of the home treatment on functional development, the type and methods of home treatment given in addition to the level of follow up provided by the physiotherapist to the family to ensure adherence of the home treatment.

\subsubsection{The Effect of Home Treatment on Functional Development}

After their identification of the framework of the home program in 2006, Novac had assessed with colleagues in 2007 the impact of home programs for children with cerebral palsy. The study included 20 children with hemiplegic cerebral palsy between the ages of 2 and 7. All the phases that were established in their previous study (Novak \& Cusick, 2006) were used to promote a successful approach to home intervention. A relationship between the therapist and parent were established to set goals that are satisfying for both parents and children were done through a home visit of 1.5 hours prior to the intervention for examining the environment, the agreement on practical un-stressful yet relevant goals.

The application of some agreed on techniques were also done to display therapist/patient interaction for some guidance for parents and caregivers. The families were then left with a documented home program and were revisited for feedback after one month and three months of intervention. Outcome measures were taken at baseline and after six months of intervention. Results of all three outcome measures that included Quality of Upper Extremity Skill Test (QUEST), the Goal Attainment Scale (GAS) and the Paediatric Evaluation of Disability Inventory (PEDI) had shown significant changes that were seen after the intervention indicating the effectiveness of the home program. All the assessors have been evaluated for interrater validity in all the assessment tools, which suggest a decreased level of bias. The sample size, on the other hand, is considered small and had only included hemiplegic patients which are another factor that would affect the power efficiency of the study. In addition, the intervention section had shown that feedback visits were made after 1 month and three months after the treatment had begun which may be considered very limited follow-up in relation to the total number of 6 months of intervention. The study had assembled a very successful pilot study of the protocol and outline designed by Novak and 
Cusick (2006), and future studies would expectedly overcome the minor defects seen in this study.

Halvarsson et al. (2010), on the other hand, aimed to assess the views of parents regarding their performance of stretching as a home program. Fifteen parents were interviewed regarding their experience with stretching which is the number of parents that were recruited until saturation was reached. A general category emerged from the data that the parents felt that they were developing in the child's life from having authority upon children in their younger age into becoming the coach that guides the child into proper stretching as much as required. Parents also expressed stress that the parents face during everyday responsibilities in as well as caring for a disabled child. Other subcategories had explained the prerequisites that the parents needed before applying any stretching or other handling techniques for their children that included education for the parents and psychosocial support.

Parents reported that previous information regarding stretching and mobility had shifted greatly after having children with disability. In mobility as a subcategory, the parents expressed different views that depended on their child's mobility level. The case of helping children with their stretching programs seemed less strenuous for parents of children with higher levels of mobility, which was expressed to be important for their child to achieve full range of motion that would help to promote more independent mobility in the future. Others thought that it is a step to prepare them for other exercises that require an improved range of motion. Other parents that had less mobile children have expressed a higher level of stress felt upon providing the stretching exercises and doubted its benefits. In another subcategory that was time parents have indicated for younger children stretching was carried out through play and other activities, therefore, the child and parent did not feel that it consumed a lot of time (Halvarsson et al, 2010). Many have expressed that $15 \mathrm{~min}$ of stretching would seem the most suitable time a longer duration that would exceed half an hour would be considered strenuous. Children of older age required set schedules and sessions to perform their stretching. Time consumed for stretching had sometimes put the parents in a torn position between wanting to spend time with the rest of the family and spending time with the disabled child alone (Halvarsson et al., 2010).

Parents have expressed that the best way of coping through the stretching session is communication between parents and children (Halvarsson et al., 2010). Parents used the 
verbal encouragement in addition to the explanation of the importance of the stretching exercise for the child. On the other hand, the verbal and non-verbal responses from the children guided the parents through providing the most suitable level of stretch in order to achieve elongation without provoking spasticity. The results of this study could be of great help in overcoming the obstacles that could prevent the parents and children from adhering to their needed home stretching. Many obstacles that may cause a very high impact on the benefits of the home treatment that could be easily avoided with some education, guidance and support for parents in their everyday life demands especially when caring for a child with cerebral palsy (Halvarsson et al., 2010).

Piggot et al. (2002) have also decided to assess the experience of parents that are delivering home therapy for children with cerebral palsy. Eight parents and 4 therapists were interviewed and observed during sessions. Participants were reviewed repeatedly up to 4 times until saturation had occurred. It was found that parents experience a couple of phases when participating in their children's treatment programs. The first phase of coming to grips with their child's condition the parents did not see gains in response to their and were not able to perform the tasks to enhance the development of their children. This included phases of facing the facts of the child's condition and severity. Parents in that phase had also felt a state of un-readiness to cope with the special needs of their child and have shown a large need of support from people surrounding them. Grief and shock was also part of the coming to grips with the condition in addition to guilt and denial that usually interfered with the commitment to providing the home care.

A strategy of survival was the following stage where the parents tried to limit the load and provide as much care possible without overstressing their lives and the lives of the child's siblings. The breakthrough was the following stage that was reported by all the parents in the coming to grips phase it was the stage that usually followed seeing progress in the child's improvement that encouraged the parents into the next phase. This then took the parents to the second phase of striving to maximise. This phase had shown stronger abilities to cope and provide the home treatment, especially when getting the support needed from people and therapists around them. The partnership that should develop between the parents and therapists depend on many factors to be successful. It initially depends on the parents' participation in the process. At the beginning of the coming of the grips phase, the parents are fragile and are still dealing with the newness of the situation that interferes with the parents' ability to enter the partnership. 
According to that, the parents in their initial phase are not able to commit to the home program which is an important issue that needs to be appreciated by therapists. It is essential to keep watch for signs of overcoming this phase in order to have the parents' full motivation and participation. Drawing attention to the gains that the child is making in addition to consideration of parents' goals is the key for providing short-term steps toward achievement. In addition, building a relationship of trust with the parents through listening and promoting support from surrounding people leads to understanding the parents' needs. Less support was found to lead to difficulty in coping with the activities required in the home program, therefore, such parents' in such conditions would benefit more from a more supportive intervention and less demanding home programs. Other findings of the interview were that most therapists did not prefer to share information regarding the severity and significance that the children had especially in the early stages. This was in contrast with the therapists' desires for high levels of information sharing.

In addition, parental goals that were found to be detrimental to the child were overlooked by the therapists, which indicates the dominance of the therapist over the treatment process. Therapists have reported that the most compelling challenge is to create a program that would satisfy the therapists at the same time as being as flexible enough fulfil the different needs of the parents in their coping journey in addition to providing the child with the highest possible degree of benefits. Although this study reflects the views of parents and therapists, the sample had only assessed a small number of parents that were of a high level of commitment to the home program. A wider and larger sample may reveal higher variability in views and the coping obstacle of parents with different circumstances.

Katz-Leurer et al. (2009) had assessed the effect of a home-based functional exercise approach designed by therapists for six weeks compared to the control group that did not receive any therapy and had just carried on with normal daily activities, school and sports. The assessment was done at baseline, after six weeks of the intervention and at 12-week follow-up. No significant difference was seen between the groups in any of the outcome measures. Some positive changes were seen in the functional reaching test in addition to the improvement in the speed of the time up and go test, however, none of these changes was significant.

The study (Katz-Leurer et al., 2009) on the other hand had indicated the feasibility of the home program with adherence noted in 9 children out of the total 10 in the intervention 
group. This high percentage of adherence was thought to be due to the simple exercises that were instructed by the therapists who intended the home intervention to be as simple and manageable as possible without being boring (Katz-Leurer et al., 2009). The authors had also provided weekly phone calls with the therapist to allow feedback, support and encouragement for both the parent and child in order to enhance better adherence to the home program. A diary was also provided for each child for written feedback regarding the performance of the home program daily. Another aspect of the study was to assess the short-term effect of the task-oriented exercise. The results have shown some changes that although were not significant but were maintained even after 12 weeks' follow-up. The tasks were influenced by the activities of daily living that required the child to carry his/her weight whether in standing from sitting or stepping up which are very demanding activities. Results may have shown significance if maybe the exercises were slightly strongly correlated with the outcome measures (Katz-Leurer et al, 2009).

The authors (Katz-Leurer et al., 2009) have argued that the getup and go-test are used to improve balance in adults and it is the reason for choosing sit to stand as one of the home exercises. Sit to stand exercises are correlated with trunk balance however other trunk strengthening exercises would have supported the effect of sit to stand in the short duration of the intervention and would have shown more significant results. Other than the choice of exercise, the duration in which the child practised at home could be considered slightly inefficient for the expected change required (Katz-Leurer et al., 2009). The child was expected to perform as many repetitions in a minute time. Repeat the exercise as much as possible. Instructions that included clear, longer duration, and are expected from the child daily may have affected the results more significantly compared to what was done. The authors attempted to provide simple and easy home interventions as an attempt to allow better adherence however it could be argued that the perhaps the intervention was instructed to be too simple to show effect (Katz-Leurer et al., 2009).

Another study by Chen et al. (2014) had assessed the application of home program using a portable rehabilitation robot. Twenty-three children with cerebral palsy level I, II, III of GMFCS were included connected to the therapists in the clinic by an audio-visual web camera that provided feedback and technical support between the patient and clinician. Similar to Bryanton et al. (2006) this study had also included the use virtual reality or video games that required the child to perform movements that were designed by therapist according to each child's needs. The feedback on the child's performance was sent to the 
clinic and changes were done on the program via the internet according to the child's progress. Adherence to the home program was found to be a 100 percent, and all children have completed the total number of session required. After six weeks of home intervention, the results have shown a significant change in the aspects of an active and passive range of motion, muscle power and stiffness.

Measurements were taken using the Modified Ashworth Scale (MAS), The Selective Control Assessment of the Lower Limb Extremity (SCALE), Paediatric Balance Scale (PBS), the six min walk test, and the Time up and Go test. The study has shown positive clinical effects of the rehabilitation robot in addition to the benefits of saving resources and providing therapy for the patients at home that was available at all times which can be time and effort consuming. It was also confirmed that providing the home program using an interesting tool would promote good commitment to applying for the program, in addition to promoting active participation for the children. However, the children included were of a small population with a specific level of GMFCS that are able to understand and perform verbal requests and commands which is not always the case with children with $\mathrm{CP}$. This technique will therefore only be suitable for a limited sample of children with CP. It should also be mentioned that such a robotic set up would require high costs to make available for a number of children to use at home although it would save the need for a therapist for each child it would still cost to provide a robot for each child at home.

A case study by Golombe et al. (2011) of a 13-year-old boy with hemiplegia had also shown significant positive results in muscle power and functional activities, even after the home intervention ended. The study had included a virtual reality video game for the upper extremity. The boy performed his exercises twice a week for 20 minutes a session for 14 months. There was no feedback or communication during the sessions and an assessment was performed after the first 8 months, and then after the termination of the intervention. No frequent adjustments or changes were applied to the intervention. Changes were seen in the muscle power of the grasp in addition to the time required to perform some activities. The outcomes continued to improve even after 2 months after the home intervention was ended. Although the study did not provide any kind of follow up or feedback regarding if the child completed hid dose of the sessions the child still showed positive changes even if it was mentioned in the study that the authors were not sure if the child had continued with his sessions in the final two months of intervention. A diary or a weekly phone or video phone call would have overcome this obstacle which may have affected the results. The study has 
also mentioned that the child was receiving on and off treatment for his disability in the clinic due to his age, this shows that the child may not have received any treatment for a prolonged period before this study and therefore, the results could be due to the child showing sudden high results due to the long previous off period. Although the results are still considered significant and of value, especially when the child is of an older age, which in general, shows lower levels of progression. Single case studies although may show significant results remain limited in the power of evidence that could be generalized.

\subsubsection{Types and Methods of Home Treatment}

In 2006, Novak and Cusick attempted to identify the main aspects required to create an outline for an effective and feasible home program. The search aimed to provide a complete protocol that included aspects of the suitable approach, parameters and aspects of commitment that would present a successful and effective home program. The literature was searched and results have shown a severe lack of evidence in many aspects of the home program. The literature had some focus on the level of commitment of parents in addition to the psychological aspects of the stress levels that parents are coping with in relation to treatment burdens. The study had indicated that as family compliance is useful in many approaches, it is not applicable to a recent family centred practice approaches that focus on participation rather than compliance. In children with cerebral palsy, the golden standard is to provide the intervention through a family centred approach accommodating the child identifying the many aspects that a family requires from an intervention such as structural concerns, active participation in addition to community engagement. According to these aspects, it is essential that the home program satisfies five phases in which should present a suitable home program. Initially, the first phase focuses on establishing a collaborative relationship with the parents. In a family centred approach, the family together with the child become the focal point, rather than the child alone.

At this point the family needs to be an active partner in the process, many families may not be familiar with this situation. For this to be achieved, therapists need to familiarise with the parents' expertise in order to influence family engagement in the approach. Factors that may control the success of this phase would depend on the therapist's skills and attitudes toward this approach in addition to the therapist's clarity regarding the parent's role in contributing to a more realistic program influenced by parents. The setting of collaborative 
goals is the second phase of achieving the targeted home program. The therapist in this phase should encourage parental decision making to enhance the capacity of parents to identify problems, priorities, and issues that influence goals. The following phase is by creating the home program and this is done by combining the families' goals and preference with the therapist's expertise.

This indicates designing a home program that is typically pleasing for the parents and not stressful for the child or family ranging for the use of adaptive equipment through to the participation in daily family routines. This would be preferable if programs were presented in a documented manner either in writing or pictorial format. Supporting the implementation of the program is the fourth phase of the program. Families need to be actively supported by therapists to ensure that the documented programs are practically feasible and are meeting the family needs. Therefore, families should be able to contact therapists for feedback inquiries and support in addition to identifying progress whether by phone or in person which may help parents in seeing the correct interaction between the child and the therapist in the home environment. The final phase of the program is evaluating the outcomes which indicate the achievement of family goals including family perspectives and observations. This model has been established to serve occupational therapy home programs, however, shows realistic and important aspects that should be considered in all fields that require continuous home interventions to support clinical approaches. It had identified the main aspects in which it is expected to promote the success of the approach that implements continuous therapy and also blends family and clinical care for the sake of better care, including better quality of life.

Bilde et al (2011) had included 9 children between the ages of six and 13 in a study to evaluate the effect of delivering a home program through the internet. The children were assessed before and after a 20 -week period in which the children performed a 30 min exercise session at least once a day. The home program was performed by interacting with a training system using flash technology. The training system provides cognitive, perceptual and motor training at the same time and identifies the child's movement through a video camera attached to the computer. The training system may be adjusted in intensity and difficulty and is controlled by the therapists that follow the children's progress through the internet. In addition, the therapists were in contact with the children and parents on a weekly basis to 
provide support and feedback which provided the influence of a personal therapist at home. The results have indicated significant changes in the functional abilities in the children.

Significant changes were also seen in the sit to stand the test in addition to the stepping test. More significant results were seen for the endurance in gait training. In general, the study assessed the level of encouragement, motivation and maintaining intensive and more lasting training compared to what is offered usually as a home program for children with cerebral palsy. The children showed a performance of 74 hours of training throughout the 20 weeks which is an average of about 30 min daily fulfilling the goal set for the children. In the study, the children performed a 30-min session daily which is much higher than what is usually provided in conventional therapy. Adherence to such frequency and duration for all the 9 children reflects a very high level of adherence and the success of the study by promoting such commitment. Compared to the results seen in Basaran et al, (2014) this reflects a 100 per cent adherence to the home program. This was justified by the subjective feedback by parents of how motivating and supporting it was to keep in contact through emails and Skype which provided a strong feeling of support and that the caregivers and children were not alone.

In addition, functional changes indicate that the correct parameters in which therapy was provided had induced motor changes and motor learning satisfying the objectives of most treatment interventions. Results of the study were limited to a higher level of children in the GMFCS which had promoted the independent interaction and performance of the activities in addition to older age. Therefore, results may not be generalised for all levels and ages of CP. Moreover, the small sample size limits the power of the results although significant. On the other hand, the study supported that the concept is applicable which should be considered to save resources at the same time as providing good levels of individualised followed up interventions at home.

Bryanton et al. (2006) included 10 children with CP compared to six children without $\mathrm{CP}$ between the ages of 7 and 17 in their study to assess the effect of virtual reality exercises on the improvement of performance levels compared to conventional exercises. The children were taught to perform a dorsiflexion movement in a conventional manner as in the specific repetition of dorsiflexion while sitting on a chair with hip and knees in 90 degrees and in long sitting. Each child then repeated their performance using a video game that promoted Virtual Reality (VR) movements that are similar to kicking a coconut to reach the highest level using 
only the dorsiflexion. The children showed more excitement and motivation during the VR performance. Parents have also reported that the children were excited to perform the VR activities and would be more committed to performing such exercises in that manner at home compared to the normal home program.

The results of this study indicated slower performance of dorsiflexion in the VR activities compared to the conventional approach as the children were required to perform the activity with more concentration steady starting point and stronger performance of the movement to reach higher goals, the game also took a while to allow a second repetition which indicates that the children have performed the activity with more concentration and quality promoting selective motor abilities. The study, however, did not provide an outcome measurement tool to measure any change in performance and had focused only on evaluating the level of excitement and motivation of both types of activities. The study focused on assessing the reaction of the children to the intervention in order to promote more adherence to the home programs. No purpose was explained regarding the recruitment of children without CP. In addition, the population of the study is relatively small and the ages of children do not promote generalisation of results for all ages and neither does the GMFCS levels of the population which only includes level I and II.

Harbourne et al (2010) had assessed the effectiveness of the home program when compared to clinical intervention. In his study, 30 infants were included and divided randomly into the two groups. The outcomes were GMFM and Postural Control Measures. The study had found no significant differences between the two groups in both outcome measures. However, the infants included in the study were partially at risk of CP and partially were diagnosed with $\mathrm{CP}$ which could have highly affected the results. The children, therefore, do not show similar baseline levels and would develop differently if they had CP or were simply just delayed. The study had discussed that it made no difference when focusing on a specific function if the intervention was delivered at the clinic or home by the parents as both groups have shown significant changes in GMFM scores. This result could have been easily reached through the maturation of the infant rather than as an effect of the treatment delivered in either approach. The results highly supported the effect of the home intervention and how it achieved similar results to the clinical intervention, however, no specific monitoring was provided to the home program. The home treatment consists of aspects such as playing handling and positioning, which was advised by the therapists that indicated minor trunk support in various positions. The home treatment was intended to be delivered daily 
with no specific parameters or monitoring that reported the adherence to the treatment rather than the correct delivery of the components. Both treatments were delivered over 8 weeks with an assessment performed at baseline, after one month and at the end of the $8^{\text {th }}$ week. In the discussion the study had mentioned that when assessing the development of only the infant with $\mathrm{CP}$ in both groups results had shown that the children in the clinical intervention group have scored higher changes compared to the children in the home intervention group which does indicate that professional intervention provides more effective outcomes moreover these results indicate that baseline levels of the group are not equal and a risk of bias is present and would affect the level of evidence of this study.

Weindling et al. (2007) had assessed the effect of different parameters of treatment including the association of home program with the clinical intervention. For 34 participants that completed the study, no significant difference was seen for groups who received additional intervention including home treatment. The study had assessed intervention via three groups where patients had received extra session per week by a physiotherapy assistant. The second group received standard physiotherapy and the third group received standard intervention in addition to the home visit from a family support worker.

Although no significant difference was seen between the group in the objective outcome measures, parents had reported some changes seen and benefits were gained from the intervention which shows a discrepancy between quantitative and qualitative results. Additional intervention was provided in this study by a physiotherapist assistant once a week with no additional information found regarding the nature of the intervention or the standard intervention being received in the first place. In addition, the home visits were for psychosocial purposes and did not include any physiotherapy intervention or exercises, therefore, it is believed that although in the results no significant changes or differences were seen between the groups it could not be generalised that additional intervention for the children with cerebral palsy is not beneficial. Various parameters could be applied in the intervention for children with cerebral palsy either as a clinical treatment or a home program, therefore, it is very difficult to generalise results from a single study with specific therapy and outcome measures to conclude the ideal approach of intervention parameters for a sample that includes a large variety of symptoms and diagnosis such as cerebral palsy. 


\subsubsection{The Level of Follow Up and Adherence}

Basaran et al. (2014) wanted to assess the adherence of parents to the home program by providing a survey of closed-ended questions relating to the level of adherence and the causes behind them. In addition, the authors have assessed the level of depression, anxiety and burnout using the Beck Depression Inventory (BDI), the Beck Anxiety Inventory (BAI) and the Maslack Burnout Inventory (MBI) respectively. A relation between the GMFCS levels of children and the level of adherence to the home program was made to justify the results found. Poor adherence levels were found to be $34.7 \%$ of the total number of the 133 caregivers of children recruited. Adherence levels were considered low in this study if the application of the home program was less than once a day.

These results are mainly based on statements of caregivers which in some cases are influential and could have been overestimated. Therefore, poor adherence could be much higher objectively in real life. Some factors that were expected to affect adherence were evaluated in this study which included educational status and employment of the caregiver in addition to total family income was found as a non-predictive to adherence. Other factors such as the age of the child that indicates the duration in which the child has been disabled in addition to the weight of the child that showed significance in univariate tests but were not found to be independent predictors of adherence. The factor that was found to be predictive of adherence in this study was the severity of the cases. The lower the level of GMFCS was the more adherent the caregivers showed to the home treatment.

On the other hand, the type of CP or the associated conditions such as seizures and visual complications were not seen to be predictors of poor adherence (Basaran et al, 2014). Results have revealed moderate to severe levels of depression and moderate to severe levels of anxiety in caregivers included in this study. However, none of these factors influenced the levels of adherence to the home treatment. Burnout, on the other hand, was found to be higher in the poorly adherent population. This indicates that neither anxiety nor depression would prevent the caregiver from adhering to the home program. However, difficulty in overcoming stress and exhaustion would result in the caregiver failing to adhere to the required home program. According to that many factors that would increase demands upon the parents would at the end lead to burnout and therefore lack of adherence however none of these factors (Number of sibling, other siblings younger than three years, presence of other siblings with $\mathrm{CP}$, presence of other family members in need of care) that were predicted by 
the authors have shown no significant results, which indirectly affect adherence (Basaran et al, 2014).

Given the fact that only parents that feel burnout have a direct correlation with low adherence and not depressed or stressed parents could indicate the phases in which the parents go through to finally accept the disability of their children and therefore show less adherence. In this case as suggested by the study (Basaran et al., 2014) more parent psychological support should be offered as soon as the diagnosis has been made. In addition to that parents should be more educated about their child's disability and level of prognosis expected to provide a clearer vision of what is expected from the parents and children to avoid future burnout and moreover less adherence.

Taylor et al. (2004) addressed the factors that influenced the adherence of parents to the home program. The article interviewed 11 patients with cerebral palsy and their parents. Factors that influenced adherence to the home programme were categorised into environmental and personal factors. It was found that emotional support from parents would provide encouragement for performing the home program. Availability of equipment was another environmental factor that induced adherence. A logbook had also been mentioned to induce adherence as it counted as the patient being supervised and followed and therefore the patients felt the obligation to perform their exercises and report them in their logbooks. Similarly, the visiting therapist had also served as a strong motive that kept the patients and their parents on track of their exercises.

Keeping the exercises concise and simple is another major factor that motivated the patients to commit to their program. As for personal factors the patients were found to be adherent when they had the choice of deciding to participate or not they were also found adherent when they were motivated to reach a goal in improving their abilities and independence. The patients were also found committed due to the challenge they felt up to, and the progress they felt they were achieving. In the discussion, the authors had emphasized the effects of the factors that were reported by both the parents and patients. The study had summarised a number of factors that are understood to be important in almost all home programs for children with cerebral palsy. Although some factors can be modified and others should have more focus depending on the children age, level of gross motor function, and cognition level; however, these main factors assemble an important base of factors that should be present to induce adherence to any home program. 
In another point of view, Wang et al. (2013) assessed the effect of a sensory influenced home program in promoting compliance and motivation to improve gross motor abilities assessed using the PEDI. The home program consisted of the performance of a single exercise (sit to stand) in different parameters of resistance and repetitions either in an intervention group of Pattern Sensory Enhancement (PSE) music or in the control group of the same exercise with no music which included 36 children altogether. The clinical group had shown some significance in gross motor capacity but no significance in daily activities, walking speed, or strength. The music was sought to provide speed and facilitate faster movement and earlier seat off in sit to stand. It was believed in the study that PSE music had an effect on movement time, increase extensor power of lower extremity and improving the smoothness of the centre of mass trajectory in children with cerebral palsy when compared to exercising without music. The same effects were also believed to be seen in on motor control. The music was also expected to make the children feel less fatigued and promote longer motivated performances as the unpublished pilot study had indicated sit to stand exercises to be non-motivating and promoted very low compliance. In the results, the music was found to show improvement in motor capacity but did not necessarily translated into capability and performance. In some aspects of the PEDI scale, the non-music group had shown some improvement higher than the music group, which did not agree with the study's hypothesis. Many factors could have affected the results including the sample size the level of severity of the disability in addition to the duration of the intervention and other parameters. 


\subsection{Conclusion}

In almost all of the studies assessed within this systematic review, the home treatment had shown promising levels of change in the developmental level of the children with $\mathrm{CP}$ and had shown high levels of maintenance even after up to 4 weeks follow up.

The best way indicated by therapists to encourage parents to adhere to the home program was to include the parents in the goals set for the home treatment. This was by including the parents in the choice of activities and exercises required. In addition, it is an important factor to facilitate simple and manageable amounts of physiotherapy treatment and exercises in order to avoid stress put upon parents, which may lead to low adherence. Another important factor inducing adherence was the education of parents in order for them to understand the purpose and the importance of applying the home treatment. Follow up had played an important role in promoting adherence in the treatment applied at home whether due to encouragement provided to parents or due to the correction of incorrect application or updates provided for intervention provided by the therapists. Follow up had also promoted the availability of the therapists for any issues or enquiries that the parents may have.

Physiotherapists must appreciate the pressures applied on parents of children with cerebral palsy and therefore must understand the phases in which the parents go through when getting to understand and apply the physiotherapy treatment at home in order to create the most suitable program for each child and family.

Different methods may be used in applying home treatment depending on the goals set including video games and virtual reality methods in order to encourage children to engage in treatment. However, intensities and types of home treatment remain highly dependent on the developmental needs of the children and the level in which the families can manage.

Different methods of the factors promoting adherence to applying the home treatment at home were investigated in the literature. This research will evaluate new methods of education that include personal training sessions tailored to apply the home treatment in addition to personal weekly home visits to the families at home in Kuwait. The aim is to investigate if closer one to one education and personal home visits would induce more adherence and therefore improved outcomes in Kuwait. 


\section{Chapter 4}

\section{Research Design: Exploring Methodological Approaches to Establish a Research Framework.}

\subsection{Introduction}

In the previous chapter literature that is relevant to the management of children with $\mathrm{CP}$ and further home care have been critically appraised. Therefore, there is a need to describe the local physiotherapy practice in the management of children with $\mathrm{CP}$ to address the aims of this research. According to that, there is a requirement to perform an evaluation of practice. Methods of clinical governance will be further explored in order to identify the best method suitable for the objectives of this research.

Within the provision of healthcare, clients or service users expect to have a level of confidence in the services they are accessing. To provide reassurance to the client, the professions must not just offer a high-quality level of services but should provide the necessary evidence to demonstrate the quality of care being provided (Sim \& Wright, 2000). Furthermore, skills and knowledge that are used to provide services should be justified in the shared body of professional knowledge (Portney \& Watkins, 2015) and in the era of evidence-based practice to justify the expenditure of service provision (Portney \& Watkins, 2015). Research is the procedure in which professions generate evidence through testing and validating theories in addition to the examination of the utility of assessment tools and procedures (Kielhofner, 2006).

Obtaining evidence of clinical practice and quality of service provision through a robust and knowledge-informed research paradigm should follow a systematic and principled pathway for solving healthcare problems across all service provision (Graham et al., 2009). It is systematic due to the need of following specific a sequence of processes; it is also principled due to the explicit rules that research must follow, which is known as the method (Polgar \& Thomas, 2013).

Current research approaches according to Polgar and Thomas (2013) may be referred to as the basics of scientific methods of the $16^{\text {th }}$ century that included: 
- Scepticism" is the right to doubt and analyse any statement or proposition.

- Determinism is the term that refers to all events of the world to regular laws and causes.

- Empiricism refers to the obligation that all enquiries should be conducted through observation and confirmed through experiences.

However, in recent years these core scientific principles were simplified in a manner that would be consistent with the term positivism, which is a natural view methodology similar to another methodology known as reductionism (Polgar \& Thomas, 2013). They begin with observation, which can be expressed as either verbal descriptions or sets of measurements. That must be translated into descriptive statements and measurements to be understood and furthermore replicated by other researchers. The key factor for a successful observation is accuracy replicability and should not necessarily include complex instrumentation. Observations from the factual basis of scientific knowledge when they are appropriately summarised and observed by others (Polgar \& Thomas, 2013).

The positivist paradigm is what most scientific research has been carried out with. They employ methodologies that are based on the principles of the scientific methods that are quantitative, where the researcher reduces the data collected to numbers that are then analysed using statistical and mathematical techniques (Bruce et al., 2008). Results provided by such methods are considered powerful tools in producing reliable and valid evidence that is demonstrable, logical and mathematically sound. According to that principle, results generated from such research could be viewed as being generalizable and replicable. In some situations, the positivist methods are considered too objective to measure the social world that cannot be studied the same way as objects in the natural world. Furthermore, in other situations, a positivist approach may be ethically non-acceptable with respect to research on patients or humans in general (Saks \& Allsop, 2013).

As for the interpretivist paradigm, it was developed due to dissatisfaction with positivism. Weber in 1947 had argued that actions could only be understood in terms of their meaning for the people taking action. On the other hand, meaning can only be understood in the context of values, culture and mores of that time. According to the various qualitative methods being developed, thereafter, it also aims to study how individuals themselves made sense of their own world (Pope et al., 2000). The knowledge collected through this approach 
is subjective, partial, and dependent on social position. The methods seek to understand what a specific phenomenon mean to participants individually and how these experiences are embedded in society and culture. Qualitative methods are considered to be flexible and provide a rich description of experiences. In addition, the method also allows the collection of data in situations where quantitative methods are inappropriate, however, even though it provides valid results, findings of such methods may not be generalised. Moreover, qualitative methods are more susceptible to observer's bias and lack of focus and consensus. In many cases, qualitative research may lack validity, and therefore, they have poor replication (Saks \& Allsop, 2013).

Mixed method research is a research design that includes both quantitative and qualitative approaches in many phases of the research. As a method, the research focuses on collecting, analyzing, and mixing of both approaches in a single study or serious of studies in the belief that the use of quantitative and qualitative approaches combined provides a better understanding of the of research problems than either approach alone (Creswell \& Clark, 2011). In many cases one data source either quantitative or qualitative is insufficient and the limitations of the use of one approach may be overcome with the strengths of the other. In addition to the use of one approach such as quantitative may produce general results that are further and deeper explained by the use of a following qualitative study. In other situations, quantitative methods are used to generalise results that have been initially found through a qualitative approach (Bruce et al., 2008). Using mixed methods provides strengths to the weaknesses of either approach, therefore, it provides stronger evidence. In addition, it helps to answer research questions that are unanswerable by one method alone and provides a bridge between the divisions of researchers supporting quantitative or qualitative research (Creswell \& Clark, 2011), which are more deeply discussed further in this chapter.

\subsection{Service Evaluation}

Clinical governance is a comprehensive framework that provides authority over healthcare organisations to monitor and improve the quality of health services that was introduced in 1997 by the National Health Service in the UK (Vanu Som, 2004). This is used to ensure best practice that is based on evidence-based medicine. These authorities are used in monitoring and minimising risks, which provides systems that protect patient confidentiality, enable training and education to improve staff competency and providing 
good working conditions (McSherry \& Pearce, 2011). They also work as a link between the patient and the healthcare providers by receiving comments and listening to patient feedback (Bowling, 2014). Monitoring of service quality by the authorities is usually seen as clinical and service evaluation.

Service evaluation is a broad term that addresses more open questions that in some cases not answerable using audits alone. Evaluations may include audits to achieve a specific objective, which is usually only one element within a broader design (Curtis \& Drennan, 2013). Depending on the purpose of the evaluation it may have different methods of application including summative evaluation of effectiveness, or sufficiency or costeffectiveness (Brophy et al., 2008). An evaluation may be used to evaluate the sufficiency of a specific tool or intervention, the continuity of using a specific intervention, costeffectiveness of an intervention, and increase knowledge regarding a specific intervention (Brophy et al., 2008).

The definition of evaluation varies worldwide, and there is not a single or correct approach of designing an evaluation study (Smith et al., 2005). In general evaluation, the value and effectiveness of the services provided for a particular audience. Therefore, any evaluation must consider the meaning of value and effectiveness and how such meaning will be determined, in addition to defining what is being evaluated and the audience in which the evaluation is for (Smith et al., 2005). In service evaluations, the most powerful influence is the audience in which the evaluation is done for however in most cases it is the factor least considered (Curtis \& Drennan, 2013).

Unlike research, service evaluation provides rapid data that may be used to create practice change. Regardless of the question when performing an evaluation, the most important aspect is to use the best approach to reveal accurate readings to support response by high-quality information that complies with ethical standards that protect the patient (Twycross \& Shorten, 2014). Also, service evaluation is designed to define and judge current practice without reference to a standard (National Patient Safety Agency, 2009).

In the UK, service evaluation allows the researchers to bypass prolonged systems of ethical approvals and therefore, in agreement with Twycross and Shorten (2014), it is considered as a rapid data that is very useful in determining quality of service provision, identifying resource needs and to assist with identifying areas of service development (Vanu 
Som, 2004). However, it may be considered a constraint when intended to be published in scholarly journals (Curtis \& Drennan, 2013).

Evaluations are considered a must in the healthcare systems; it provides evidence and information required for decision-making, service planning and allocating resources (Twycross \& Shorten, 2014). Additionally, they are often used to improve healthcare management, organisation and delivery for patients. A common misconception of evaluations is that they may only be applied after an activity had taken place or is well established. This reflects the fact that in many vases planning an evaluation is delayed and avoided and therefore, resulting in weak designs missed opportunities for collecting valuable data (Curtis \& Drennan, 2013). The most common disadvantages of evaluations are being costly and time-consuming and may sometimes require special skills to apply (Curtis \& Drennan, 2013).

Bowling (2014) reports that service evaluations may be divided into either summative or informative evaluations. Summative evaluation is the evaluation that includes active or terminated program with the objectives of whether the program should be repeated or continued. On the other hand, formative evaluation aims at developing or improving a program while it is still active (Bowling, 2014).

In Kuwait, the Ministry of Health is responsible for the governance of both healthcare services and documentation levels of patient records. The procedure is performed with the assistance of Accreditation Canada International.

Service evaluation is a broad term that addresses more open questions that in some cases are not answerable using audits alone. Evaluations may include audits to achieve a specific objective, which is usually one element with a broader design (Gottwald \& Lansdown, 2014). Similar to research, service evaluations have both strengths and limitations that depend on the purpose, approach and context used in the application (Gottwald \& Lansdown, 2014). The category of the service evaluation influences how the study is carried out.

\subsection{Clinical Audits}

Clinical audits and service evaluation share the purpose of improving services and informing policies and planning decisions. However, they are distinguished by the prospective study. Audit is considered to be practitioner-based and is conducted by oneself or 
by one's peers to review the quality of clinical care (Bowling, 2014), unlike evaluations that are usually applied by researchers that are not part of the service giving an outside prospective service. Audits are also considered to be short cycled and allow quick changes in practice to be implemented compared to the evaluation that is usually planed on a longer larger scale that examines the impact of a new service or redesigned service delivery system (Gottwald \& Lansdown, 2014).

Additionally, a clinical audit may be implemented using different types of methods depending on the purpose of the audit itself. Chambers and Wakley (2005) have reported different types of audits of different methods of application:

- Case note analysis audit: a retrospective review of a random selection of notes or a prospective survey of what has been done for consecutive patients with the same condition, which provides insight into current practices. Looking at the history of care is considered the richest material for expanding knowledge.

- Peer review audit: comparing an area of practice with how other individual professionals work.

- Criteria based audit: comparing clinical practice against specific indicators of performance, guidelines or protocols from national publications.

- External audit: this is performed by external bodies of audit facilitators.

- Direct observation: recording what is observed in consultations or procedures.

- Surveys: with respect to set standards a survey may be carried out as a general indicator of care or for detecting a problem by using standardised validated tools.

- Significant event audit: includes adverse and critical events that have already caused harm or that are considered of risk. 


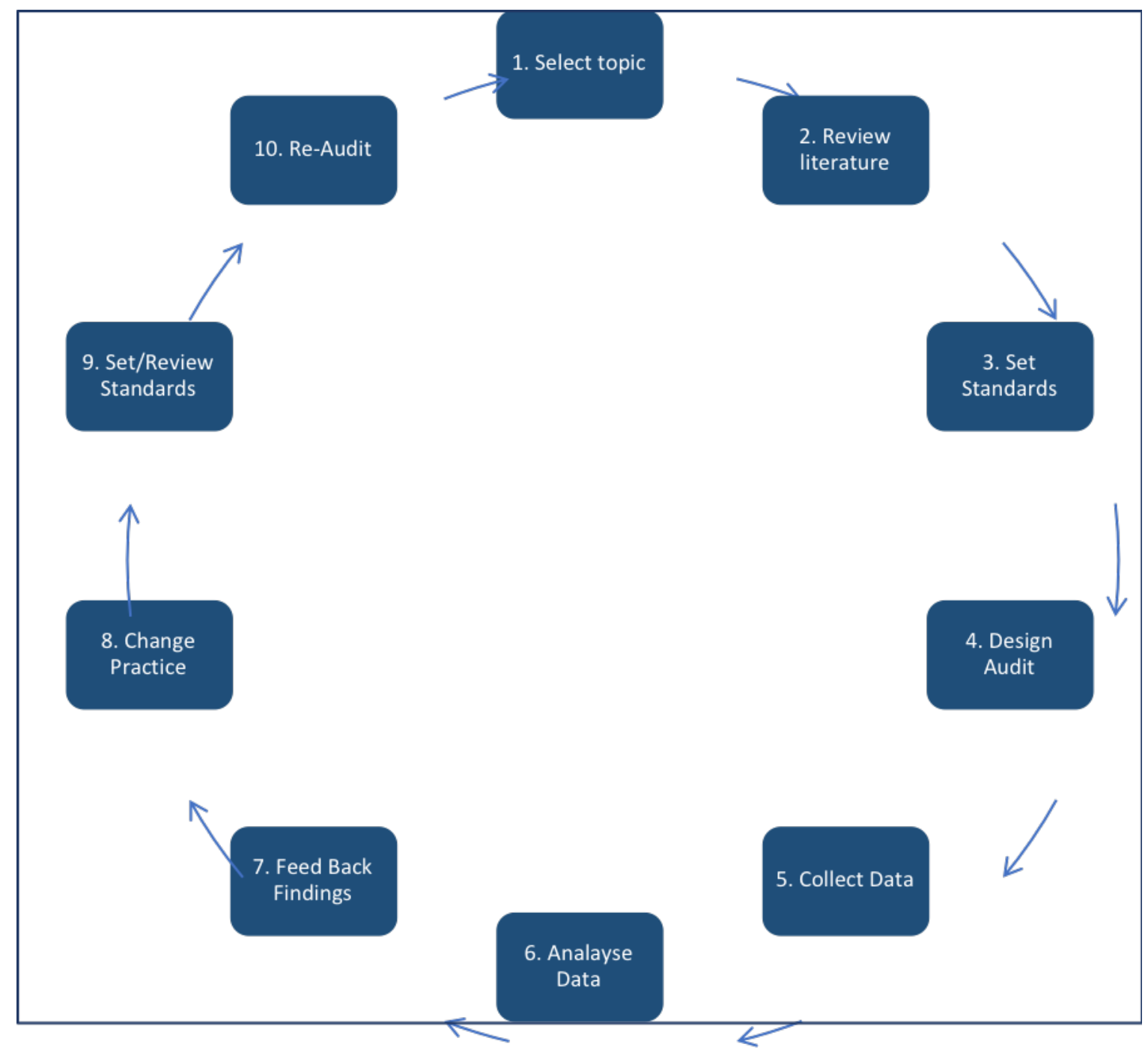

Figure 4.1 The Clinical Audit Cycle

Clinical audit is the only tool that can measure practice against a set of prescribed standards, whether they be organisational or professional (Benjamin, 2008). It could measure the structure of care that includes resources, the process of care such as waiting times in clinics in addition to the outcome of care that is similar to results of a specific intervention (Benjamin, 2008). Although audits are currently being applied as healthcare governance, it still lacks the evidence that supports its validity in improving practice (Grimshaw et al., 2001). Mostly, the lack of time to participate in the main barrier that is found to hinder its effectiveness; another barrier may be the lack of effectiveness of audits that is seen in the literature in changing already existing practice in the health care system. In other cases, (NICE, 2002) audits were found effective when feedback was delivered more intensively. 


\subsubsection{Guidelines for Clinical Audits (Fig 4.1):}

1. Selecting a topic: the first step of an audit is to select the topic by deciding what is needed to know about a service that is being provided.

2. Reviewing the literature: to learn any national standards that are used for in the same topic selected for audit, and learn the results of any previous audit applied in the same area.

3. Setting Standards: to help compare current practice in order to identify problems that may not have been recognized if no standards are set. In many cases in clinical audits practice may be compared with findings in the literature.

4. Designing the Audit: which is usually done by the key stakeholders, and should consider what data will be collected, the sample population and size, the data analysis, the feedback of the findings and finally the time limits of the audit.

5. Collecting the data: must ensure confidentiality, secure storage of data.

6. Analyzing the Data: the type of analysis will depend on the type of data and may consist of descriptive statistics, statistical tests, and qualitative analysis.

7. Feedback findings: discussing the findings with stakeholders, by identifying aspects of practice that needs to be changed.

8. Changing Practice: by understanding the causes of not meeting the standards and learning how problems can be overcome. Identifying who needs to make the changes and finally when should the changes be implemented and furthermore monitored.

9. Reviewing Standards: using the results of the audit to review previous standards or set standards for the first time in some cases that have had no previous standards.

10. Re-Audit: deciding when and how to re-audit to assess the validity of changes applied.

Clinical audit could be performed using various methods that include document searching and analysis, analysis of routine data, clinical case reviews and presentations in team meetings. Below is a comparison between audits and service evaluation:

Table 4.1 Differentiating audit and service evaluation, (NRES Ethics Consultation E-Group, 2006) 


\begin{tabular}{|c|c|}
\hline Service evaluation & Clinical Audit \\
\hline $\begin{array}{l}\text { Designed and conducted solely to } \\
\text { define or judge current care }\end{array}$ & $\begin{array}{l}\text { Designed and conducted to produce } \\
\text { information for the delivery of best care. }\end{array}$ \\
\hline $\begin{array}{l}\text { Designed to answer the question: } \\
\text { "What standard does this service } \\
\text { achieve?" }\end{array}$ & $\begin{array}{l}\text { Designed to answer the question: } \\
\text { "Does this service reach a predetermined } \\
\text { standard?" }\end{array}$ \\
\hline $\begin{array}{l}\text { Measures current service without } \\
\text { reference to a standard. }\end{array}$ & Measures against a standard. \\
\hline $\begin{array}{l}\text { Involves an intervention in use ONLY. } \\
\text { (The choice of treatment is that of the } \\
\text { clinician and patient according to } \\
\text { guidance, professional standards and/or } \\
\text { patient preference.) }\end{array}$ & $\begin{array}{l}\text { Involves an intervention in use ONLY. } \\
\text { (The choice of treatment is that of the } \\
\text { clinician and patient according to } \\
\text { guidance, professional standards and/or } \\
\text { patient preference. }\end{array}$ \\
\hline $\begin{array}{l}\text { Usually involves analysis of existing } \\
\text { data but may include administration of } \\
\text { simple interview or questionnaire. }\end{array}$ & $\begin{array}{l}\text { Usually involves analysis of existing data } \\
\text { but may include administration of simple } \\
\text { interview or questionnaire. }\end{array}$ \\
\hline $\begin{array}{l}\text { No allocation to intervention groups: } \\
\text { the healthcare professional and patient } \\
\text { have chosen intervention before service } \\
\text { evaluation. }\end{array}$ & $\begin{array}{l}\text { No allocation to intervention groups: the } \\
\text { healthcare professional and patient have } \\
\text { chosen intervention before the clinical } \\
\text { audit. }\end{array}$ \\
\hline
\end{tabular}

In consideration of the above information, a case note analysis audit is the most appropriate methodology to evaluate the applied practice of physiotherapy management of children with CP in the Physical Medicine and Rehabilitation (PMR) hospital on Kuwait. 


\subsection{Research Design}

As part of the clinical audit's cycle of performance, it is required after identifying the points of improper practice, to improve aspects that need to be changed (Knox \& Evans, 2002). In order to provide changes to an available aspect of practice, it was found suitable to apply the changes that have been created to improve practice in a pilot study, to evaluate the feasibility of the aspects of practice that have been improved. With respect to that, further evaluation of the available methods of research must be performed.

\subsubsection{Experimental Design}

Experimental research is one of the most rigorous types of research available that is usually used to assess the cause and effect of a relationship for a group of dependent and independent variables (Newell \& Burnard, 2011). It is the design usually used to assess the effect of therapy or a drug (Newell \& Burnard, 2011). Experimental designs highly depend on the variables and measurements of the study, therefore, they are highly controllable by the researcher's abilities to manipulate dependent variables in order to confirm or rule out the set hypothesis (Portney \& Watkins, 2015).

It is the design of scientific experience that plans manipulation, observation, and control which used in research in the situations that variables are controlled (Chatburn, 2011). Four methods of control are commonly used in experimental research that includes:

- Random selection and assignment of the sample.

- Matching subjects to achieve homogeneity between groups.

- Including a nuisance variable as a treatment variable.

The advantage of the randomized design is that randomization may be expected to even out any variables that may be found in one group, whereas the matching experimental design is usually implemented when subjects of the population are used as their own controls such as in before and after studies (Chatburn, 2011).

Furthermore, experimental designs are ranked from weakest too strongest according to their level of control depending on the following terms: 
- Pre-experimental: which include little or no control of extraneous variables used commonly in pilot studies.

- Quasi-experimental: which lack the full control of the variables with an effort to compensate with the other controls.

- True-experimental design: which provide full control of variables.

\subsubsection{True Experimental Design (Randomised Control)}

It has the greatest degree of control. In the randomised posttest design, any differences such as age, sex, or weight that may affect the dependent variable are averaged out through randomisation between the experimental and control groups. Therefore, the pretest is no longer needed to determine equivalence (Taylor, 2005).

\subsubsection{Hierarchy of Evidence}

The hierarchy of evidence is a core principal of Evidence-Based Practice that attempts to address the best available evidence. The higher up the hierarchy the study design is positioned, the more rigorous the methodology and hence the more likely it is that the study design can minimize the effect of bias on the results of the study. In most evidence hierarchies', well-designed systematic reviews and meta-analyses are at the top of the pyramid, followed by randomised controlled trials, and expert opinion and anecdotal experience are at the bottom (Hickson, 2013). 


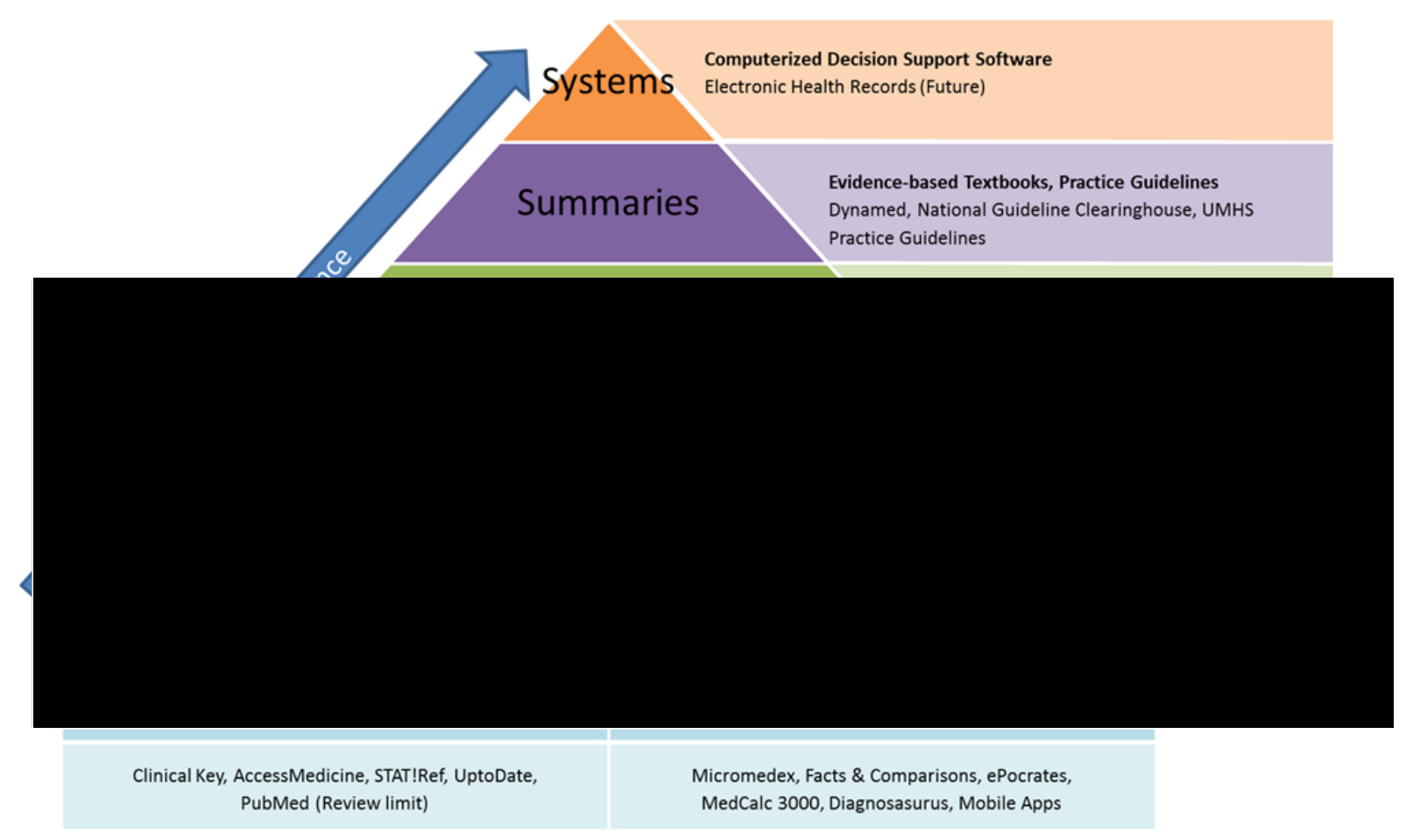

Figure 4.2 Modified from Haynes RB. Of studies, syntheses, synopses, summaries and systems: the " $5 \mathrm{~S}$ " evolution of services for evidence-based healthcare decisions. ACP J Club. 2006 Nov-Dec;145(3): A8-9.

\subsection{Non-Experimental Designs}

Non-experimental designs are considered the least in scientific rigour compared with the other designs. The validity of this design, however, depends on the method of the design itself as it varies between different types that include: cross-sectional study, case-control study, before and after study, historical control, surveys, case series, and case reports. These types of designs are considered purely observational and descriptive and are highly used in prevalence studies and may in some cases propose some associations without effectively proving them (Thompson \& Panacek, 2007). Non-experimental designs are retrospective in nature and therefore, variable cannot be manipulated by the examiner and variables of the intended study had already occurred before the study had taken place, they lack the element of control and therefore, it is very difficult to restrict potential extraneous variables, which makes this type of studies very prone to bias (Thomas \& Panacek, 2007). Terms associated with non-experimental research are used in different ways among individual writers and various fields of research in behavioural sciences and clinical medicine (Chatburn, 2011). 


\subsubsection{Survey Designs}

Experimental designs are considered to be the strongest method of research that provides the highest level of evidence; however, in some cases, it is not possible to employ such a method (Chatburn, 2011). Conditions that have uncontrollable variables, or in other cases that experimental designs may not be ethical or even cases that do not have simple causal relationship an experimental design becomes unfeasible and therefore, other nonexperimental methods are required. In similar cases, especially within the health research, survey designs are used to investigate characteristics of a population for specific variables in cases that include opinions and attitudes that concern health-related issues in addition to characteristics of a population regarding health-related variables such as utilization of health care and furthermore the collection of demographic characteristics of populations. In such conditions, the survey design serves to provide an overview of the state of health, illness and treatment patterns in specific populations. This provides essential results of prevalent causes of death or causes of diseases, which may be further developed into theories and hypothesis in epidemiology (Polgar \& Thomas, 2013). The survey is one of the most popular methods of collecting descriptive data. The survey is used to collect the responses of a group of subjects that include describing attitudes and values, levels of knowledge and experience, current practice or characteristics of this group with the intent of generalising the responses to describe a larger population (Portney \& Watkins, 2015).

\subsubsection{Questionnaires}

Questionnaires are methods of a survey in the form of a self-report, which is recorded by the researcher without observing the attitude or behaviour of the respondents. As a form of self-report the method is always potential for bias or inaccuracy; however, it remains the only direct method feasible for obtaining information that may include perception, fears, motivations, and attitudes. Questionnaires are self-administered surveys that are completed in the participant's own time, and therefore they are considered more efficient than interviews. They are also more standardised and all participants are exposed to the same questions in the same way, which may reduce interaction bias that may occur with an interviewer. Respondents to the questionnaire may take their time to think about their answers and even return to records for consultation in addition to being anonymous which may encourage honesty. Questionnaires are considered the most suitable tool in assessing modes of general 
practice in addition to attitudes and opinions even for large population samples due to the simplicity and economic nature of the tool, as it is as simple as a paper and pen or even a link through a website or email. The disadvantage of a questionnaire is that participants may misunderstand the purpose or response choices of the questions. Another disadvantage may be the rate of response, especially if distributed through the mail, therefore, even though manual distribution may be considered an outdated method it has a higher level of response compared to mail distribution (Portney \& Watkins, 2015). Electronic distribution, on the other hand, remains the easiest and the most economical. However, Boyer et al. (2002) indicate that for economic reasons, although important, should not be a factor that affects the instrument choice, alternatively other factors such as quality of the measurement tool should be considered. Furthermore, electronic surveys were found to have a much lesser return period in addition to much less incomplete response when compared to paper surveys (Boyer et al., 2002). On the other hand, Cheyne and Ritter (2001) had found that response rate to electronic surveys is much less compared to manual surveys especially with spam effect which is the service that would discard any unknown sender of mail into the spam category. It is therefore essential to find a way to overcome the spam effect if email distributions are selected. Another option would be to choose a different electronic distribution method in addition to a pre-notification and reminder message, which according to Sim and Wright (2000) had shown the highest rate of response when compared to other electronic methods of distribution that had no assisting schemes. Response bias is the major type of bias that may occur in questionnaires, which may question the validity of the method used.

According to the previous search in the different research design and with respect to the sequence of the findings and requirements of the research questions, the following methods have been selected to fulfil the objectives of each chapter of the project. Furthermore, the advantages and disadvantages of each method have been highlighted in the following table: 
Table 4.2 Demonstrates the advantages and disadvantages of the methods used in the research

\begin{tabular}{|c|c|c|}
\hline Method & Advantage & Disadvantage \\
\hline $\begin{array}{l}\text { Clinical } \\
\text { Audit }\end{array}$ & 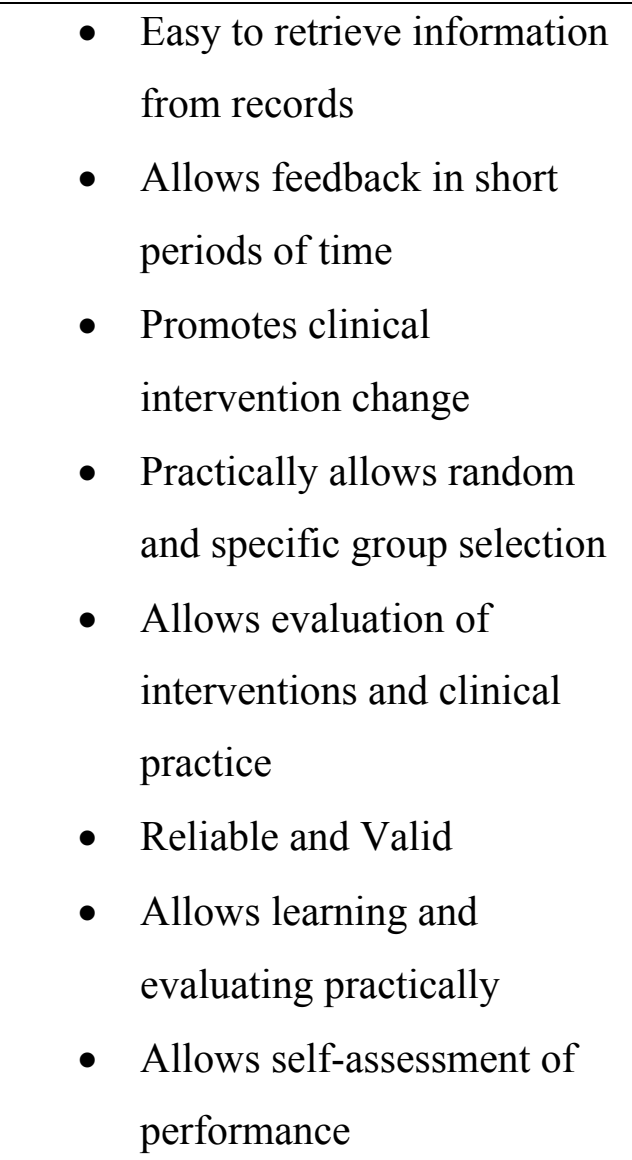 & $\begin{array}{l}\text { - Highly dependent on the quality of } \\
\text { the documentation } \\
\text { - Intervention and practice found in } \\
\text { records are highly dependent on the } \\
\text { clinical judgment } \\
\text { - Time-consuming } \\
\text { - May be costly }\end{array}$ \\
\hline $\begin{array}{l}\text { Clinical } \\
\text { trial }\end{array}$ & $\begin{array}{l}\text { - The most reliable form of } \\
\text { Scientific evidence } \\
\text { - Results may be used in } \\
\text { systematic reviews } \\
\text { - Results of trials contribute to } \\
\text { changing clinical practice }\end{array}$ & $\begin{array}{l}\text { - Time-Consuming and may be high } \\
\text { in costs } \\
\text { - Participants may not represent the } \\
\text { population properly } \\
\text { - Difficult to attain participants } \\
\text { - Does not reveal causation of results } \\
\text { - May have limited external validity } \\
\text { - Requires prolonged procedures of } \\
\text { ethical approvals }\end{array}$ \\
\hline
\end{tabular}




\begin{tabular}{|c|c|c|}
\hline $\begin{array}{l}\text { Questio } \\
\text { nnaires }\end{array}$ & 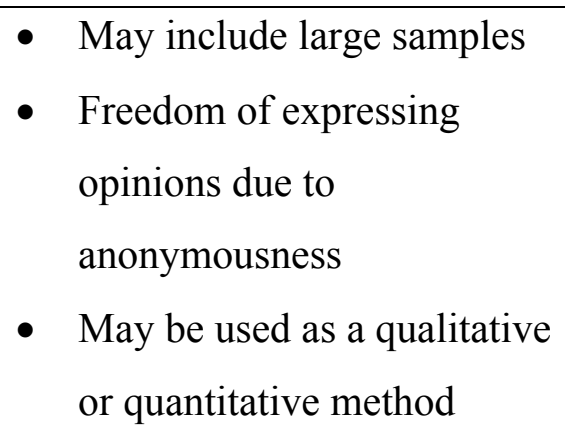 & $\begin{array}{l}\text { - May have a poor response and poor } \\
\text { completion rates } \\
\text { - Highly dependent on participant } \\
\text { level of knowledge } \\
\text { - Self-reported data }\end{array}$ \\
\hline $\begin{array}{l}\text { Online } \\
\text { Survey }\end{array}$ & 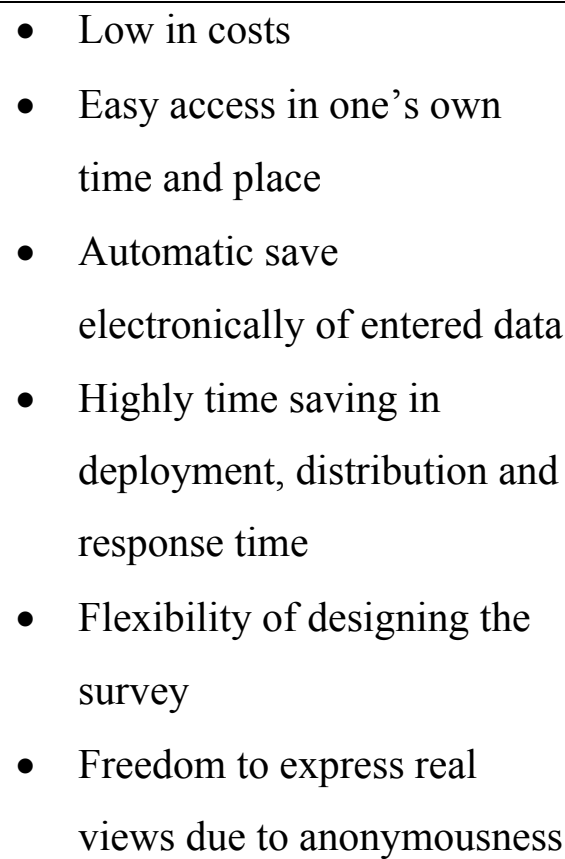 & $\begin{array}{l}\text { - Some populations may find } \\
\text { difficulty in having internet access } \\
\text { - } \text { Difficulty in locating websites and } \\
\text { email addresses } \\
\text { - May have lower response rates if } \\
\text { randomly distributed and could be } \\
\text { mistaken with spam } \\
\text { - Confusion due to unavailability of } \\
\text { an interviewer that may explain } \\
\text { questions }\end{array}$ \\
\hline
\end{tabular}

\subsection{Methods of Project Studies}

The methodologies of the 4 studies within this research were influenced and created according to the information found in the literature that were discussed in chapters two, three and four in addition to the methodologies considered in this chapter.

\subsubsection{Study 1 - A Clinical Audit: Evaluating Clinical Practice in the Paediatric Department of Physiotherapy in PMR Hospital in Kuwait}

The search for physiotherapy practice had shown a high variation in the literature. In order to understand, learn and compare the services in Kuwait to the services worldwide a clinical audit was required to assess the parameters and nature of the services delivered. A 
clinical audit was considered to be the most suitable method to evaluate and investigate the information required after searching the literature for service evaluation.

The audit was considered a suitable tool that would assess the procedures that a patient would go through when referred or walked into the centre followed by the nature of both assessment and intervention protocols. It was considered due to the short cycled nature of the method (Parry \& Watts, 2004) and the ability to evaluate the practice in the clinic against standards that are available and commonly used in the literature. In addition, as the study is done by a physiotherapist in Kuwait, the audit was considered the correct method for this situation (Parry \& Watts, 2004).

The objectives of the study had been:

- Assess the nature of the referral system used by the different hospitals in Kuwait and designed by the ministry of health to the paediatric physiotherapy centre in PMR hospital in Kuwait.

- Assess the nature of the treatment course whether it is intermittent with a two or three-month intervention course followed by an off period that may reach up to 8 weeks or continuous throughout the child's life since being referred and up to five years of age (School age).

- Assess the condensation of patients' numbers referred to the centre compared to the recourses of a number of physiotherapists available in the paediatric physiotherapy centre.

- The demographics of numbers of patients and types of cerebral palsy seen in the clinic

- Assessment of types of intervention and parameters used in the clinic

- Assessment of the nature of the documentation system used and applied in the centre

- Assessment of the availability and parameters of a home program given to patients and caregivers

The objective was to gather as much information as possible to provide a general and broad aspect that could be generalised to reflect practice in the centre. Clinical audits do not have specific search questions rather than depend on the nature of the practice being audited 
and the information required from the researcher. The information was gathered from 232 patient records and filled into sheets that had represented the information for each patient's record included in the study.

The information gathered from the audit was planned to assist in comparison to the common practice parameters of applying physiotherapy management including course duration and intervention intensities and frequencies and whether or not supporting home treatment are provided and are found effective in the literature (Ahl et al., 2005; Blundell et al., 2003; Darrah et al., 2011; Harris \& Roxborough, 2005, Salem \& Godwin, 2009) Therefore, it develops the methodology and procedures for the trial study of this research.

\subsubsection{Study 2 - A Clinical Trial: Does Providing Parents Additional support for the delivery of a Home Program Improve the Functional Outcomes of Bobath? A pilot trial Study}

The clinical audit had been intended to help in understanding the practice found in the workplace and allowing comparison to best practice in order to improve the services. According to that, after some limitations and aspects of improper practice had been identified through the audit in the department further corrections and changes in practice had to be implemented. A pilot clinical trial was then required to assess the level effectiveness of the changes that had to be made to the current practice. A feasibility study was intended to help evaluate the feasibility of the pilot study and to identify any obstacles that may occur during the pilot study that could be avoided. It was to be performed prior to the pilot study.

A trial was the methodology indicated for this chapter to assess the effectiveness of new intervention parameters when delivered using the home program provided by caregivers.

The trial in this research had been a part of a larger clinical plan of the whole research which had a role in defining and creating the other factors of the methods and procedures of this chapter including design, population, controls, randomisation, blinding and duration (Fitzpatrick, 2006). 


\subsubsection{Study 3 - A Survey Measuring the Level of Satisfaction of Parents with the Pilot Randomized Controlled Trial}

This study was intended to assess the satisfaction of the parents and caregivers with the trial that they have been a part of which would indicate the validity of the trial compared to the information sheet that they have read, understood and agreed upon at the beginning of the study.

A qualitative method was thought to be useful in this part of the research in order to achieve a high number of opinions regarding various parts of the trial that would include all participants in the study. A mixture of open and closed questions was created to ensure mixed methods of the framework. This would ensure gathering and identifying deeper responses as well as closed-ended questions.

\subsubsection{Study 4 - A Survey to Evaluate the Views of Physiotherapists on Bobath for Children with CP:}

The fourth and final study in this research was developed to support the trial and parents' satisfaction questionnaire. In order to improve and change the practice the views and opinions of therapists working and providing care must be considered. _For further generalisation, the survey was intended to assess the views of the therapists working in other work sites as well as therapists that are working in the same centre as the trial. Further opinions included to support and compare views of therapists all over the country. This was thought to reflect a higher reliability level. Due to the large number of participants in various sites, the survey was created online using an online survey website known as Survey Monkey and was distributed using a link via emails sent to the official emails and websites of the included departments. Survey Monkey is a software established in 1999, to provide online surveys that may be customised to choose question types and layout. Online surveys are found to be easier to distribute and reach the required population with minimal efforts. It provides a higher level of freedom by allowing participants to provide opinions anonymously at their own time and privacy. The software provides a smooth transition from one question and part of the survey to the next ensuring easy participation and simple click procedures.

An application on smartphones (WhatsApp messenger) which is an instant messaging service for smartphones that uses the internet to send text messages, documents, PDF files in addition to voice and video calls and many other features. It was also used to send the link to 
as many participants using the application as possible. It should be mentioned that this application is a very popular application used very widely in Kuwait.

The previous research methods were considered to investigate the different objectives of this research in order to answer the questions raised and fulfil its set aims. 


\section{Chapter 5}

\section{Evaluating Clinical Practice in the Paediatric Department of Physiotherapy in Physical Medicine and Rehabilitation Hospital in Kuwait: A Clinical Audit}

\subsection{Introduction}

As explored and described within the previous chapter (see 4.3), a clinical audit approach is a tool that can be routinely used to assess the quality of the health care services being provided to patients (Vanu Som, 2004). Therefore, it was selected as the method to evaluate the physiotherapy services being provided for children with cerebral palsy in a specific rehabilitation hospital in Kuwait.

In Kuwait, the Physical Medicine and Rehabilitation (PMR) hospital is the main and only specialised hospital that provides physiotherapy services for children with cerebral palsy to achieve as much independence as possible. Physiotherapy services for children are found in other hospital departments in Kuwait, however, such departments are limited in the variety of services with limited staff resources that cover a smaller geographic area of residents. Cases that are referred to the department include many types of $\mathrm{CP}$ in different preschool ages. Children are referred to the department of paediatric physiotherapy through paediatricians or neurologists and would then be seen by a doctor of physical medicine. A decision is then made if the child is in need of physiotherapy treatment in addition to further investigations. Decisions are then made for regular or intensive blocks of treatment, and according to that, the child is then referred to a physiotherapist to receive treatment.

Conventional treatment is a course of two to three months' blocks of treatment that could be extended depending on the need of the child, which is a decision, made by the physiotherapist. Sessions are usually given as twice or three times a week for a duration of 60 minutes per session. After the block of treatment, the child is seen again by the physician for evaluation of progress and is referred again to a physiotherapist to begin another treatment block. A gap between blocks of treatment may extend up to 2 months depending on the list of patients waiting to receive treatment.

Intensive treatment blocks in the paediatric department in PMR would consist of a six weeks' block of treatment providing 1 hour and 45 minutes of physiotherapy sessions daily in addition to other interventions such as hydrotherapy, speech therapy and occupational therapy on a daily basis depending on each child's needs. 
Children receiving physiotherapy treatment in the department are expected to maintain a level of commitment to the treatment sessions. Continuous absence from treatment sessions would lead to the cancellation of the treatment course. The child and parents would then be required to be seen again by the paediatrician and referred back to physiotherapy treatment. Waiting periods to see a paediatrician would vary depending on the waiting list of the outpatient department which may reach up to 8 weeks. The patients are then required to wait to be seen by a physiotherapist and start physiotherapy treatment which may take up to 8 weeks.

During the treatment courses, the physiotherapist is expected to document the services being provided including the assessment tools and procedures, goal settings and level of follow-up to the patients and parents at home.

\subsubsection{Aims of Clinical Audit}

The purpose of this audit was to evaluate the level of achieving set goals for children with $\mathrm{CP}$ in the paediatric department of PMR hospital which reflects the quality of intervention provided. The audit was also intended to evaluate the education of caregivers regarding the importance of home treatment as well as follow up of home treatment application. An audit that evaluates such aspects was not conducted previously in Kuwait.

\subsubsection{Study Objectives}

- To assess the referral strategy with the types and numbers of treatment courses provided for the patients in order to evaluate accessibility to physiotherapy services that are provided to children with cerebral palsy. This would allow to understand the appreciation that the parents have towards the available services.

- To assess the cancellation policy and frequencies for patients. This would reflect the level of commitment that the families have to the provided physiotherapy courses.

- To assess the level of reliability and validity of the outcome measures used to assess the treatment effects. This is expected to justify the level of goal achievement and if the correct outcome measures are being used to evaluate the problems that each child 
has in order to provide the best physiotherapy intervention and moreover achieve set goals.

- To assess the level of set goals achievement in the department to evaluate effectiveness of the intervention provided in addition to identifying the limitations that are affecting goal achievement.

- To assess the education process for the families regarding the needs of the child with $\mathrm{CP}$ in addition to the role and importance of home treatment in the supporting of clinical physiotherapy treatment.

- To assess the availability of an objective method of follow up to the application of home treatment provided to the families.

\subsection{Methods and Material}

This clinical audit is based on patient data collected over a three-year period (2010 to 2013) at a single clinical location based in Kuwait. This section of the chapter will provide the information regarding the methodology employed for this service audit based study.

\subsubsection{Study Location and Population}

A retrospective study was performed on the population of children with cerebral palsy that included 232 children that have received services from the clinic between September 2010 and Sep 2013. The timeframe was chosen according to the most recent data available at the time of collection in 2013, which extended back to include a large number of participants to ensure that the results found from the audit reflect the practice of management of children with CP in the department. The data was collected by the experimenter manually for each patient record using a data sheet of 30 questions to be filled in from each patient record.

In this study, an audit was performed to assess the level of services provided in a rehabilitation hospital in Kuwait. The Physical Medicine and Rehabilitation centre is the only independent rehabilitation centre in Kuwait and the largest of the four major centres that provide physiotherapy services for children with cerebral palsy.

The paediatric department had included 45 physiotherapists of different levels of qualifications. The staff ranking had ranged from 10 beginner practitioners to two practitioners, to 8 first practitioners to 10 specialists (one of whom had been Bobath trained), 
to 9 senior specialists (four of whom are Bobath therapists), and six head of specialists (two of whom are Bobath therapists). The educational level of staff would range from a diploma in physiotherapy to master's degree in physiotherapy including a bachelor degree of physiotherapy.

The children whose records were included had been referred to the department by a paediatrician. The department does not have a walk-in policy.

\subsubsection{Study Design}

A retrospective audit design was used to retrieve data from clinical notes, medical and physiotherapy documentation. The audit had included all children with cerebral palsy that have received physiotherapy services in the clinic in that time frame.

The audit was chosen to assess the nature of the services delivered including the parameters of the session's duration and frequency per week in addition to the nature of the approach of intervention. The assessment also included the objectives of the treatment provided and the goal setting in addition to the outcome measurement tools used to assess progress and goal achievement.

\subsubsection{Inclusion and Exclusion Criteria}

As the data included in the audit was obtained through a retrospective sample of a specific type of disability in a specific time frame. The inclusion criteria consisted of:

- Patient diagnosed with cerebral palsy

- Patient had been receiving treatment in the paediatric physiotherapy department of PMR hospital

- Patient with a referral to the department between the years 2010 and 2013

All patients that fulfilled the criteria were included in the audit, and no exclusion criteria were available. 


\subsubsection{Ethics}

Ethical approval for a clinical audit was not required according to Bowling (2014), however, as this audit was performed in Kuwait and that results of this audit was being published. The approval of the clinical audit was sought from the ministry of Health of Kuwait (Appendix 4) following the advice received from the College of Human and Health and College of Medicine Research Ethics Committee (Appendix 5).

\subsubsection{Data Collection}

The audit specifically assesses the physiotherapy services provided for children with cerebral palsy between the ages of one and five years. Two hundred and thirty-two referrals of children with different types and levels of cerebral palsy that were receiving physiotherapy services were identified from the administration system or equivalent in the hospital. Data was then gathered from patient records that were retrieved from the patient records department. An Audit Data Collection sheet was then used (Appendix 6) to gather the information from patient files and records.

Aspects that were investigated by the audit were:

1) The variety of referral methods and appointment services that would ensure minimal waiting periods for the services

2) The different types of $\mathrm{CP}$ cases seen in addition to the different ages of children receiving treatment in the department.

3) Parameters and intensities of treatment sessions

4) Treatment and documentation skills of therapists

5) Home program and patient education

A pilot study was implemented at this point to evaluate the feasibility of the procedures of the audit. The pilot had also aimed to detect any defaults or limitations of the collection tool used to collect data from the patient records. The pilot had helped to identify any additional information that needed to be gathered from the records in addition to identifying unnecessary information that might need to be discarded. It had also helped in creating a time limit of the data collection period. The study had included the records of five patients, whose information was gathered manually using the data collection result sheet. 
After the pilot study, the main audit took place that had included 30 different variables that were filled into an Audit Data Collection sheet with the different aspects assessed (Appendix $6)$.

\subsubsection{Data Analysis}

Data analysis was performed to analyse the information on the result sheet as quantitative data. This was explored using descriptive frequency statistics and was displayed graphically to identify mainly the prevalence and frequencies of children with $\mathrm{CP}$ and types of $\mathrm{CP}$ that are receiving treatment in the department and the frequency of treatment courses and referrals in addition to the statistics of data documentation and procedures. Statistical analysis was performed using SPSS (IBM SPSS Statistics, version 22). 


\subsection{Results}

In 2013 the audit was performed in PMR Hospital and had included the patients with cerebral palsy that have been referred to and have been receiving treatment in the clinic between the years of 2010 and 2013. Two hundred and thirty-two Audit Data Collection sheets were completed to ensure all necessary data required to meet the research objectives for this audit study was systematically collected on each of the 232 children with cerebral palsy identified via the initial search for patient records of which $42.9 \%$ were girls and $57.1 \%$ boys. The percentages of ages included were as represented in the following table:

Table 5.1 Indicating the ages and gender of the children that records have been included in the clinical audit that has been receiving treatment in the department of paediatric physiotherapy in PMR hospital in Kuwait between 2010 and 2013

\begin{tabular}{|l|l|l|}
\hline Date of Birth & Males & Females \\
\hline 2004 & $5.7 \%$ & $5.7 \%$ \\
\hline 2005 & $7.3 \%$ & $5.7 \%$ \\
\hline 2006 & $13 \%$ & $7.8 \%$ \\
\hline 2007 & $14.5 \%$ & $21.3 \%$ \\
\hline 2008 & $20.3 \%$ & $28.8 \%$ \\
\hline 2009 & $17.4 \%$ & $13.4 \%$ \\
\hline 2010 & $14.5 \%$ & $13.5 \%$ \\
\hline 2011 & $7.3 \%$ & $3.8 \%$ \\
\hline
\end{tabular}




\subsubsection{Information Regarding the Referral of the Patients}

Section A of the Audit Data Collection Tool reports the findings of the information collected related to the children with a confirmed diagnosis of cerebral palsy, to the physiotherapy clinic within the rehabilitation centre. It further explores how the department's system works in relation with the number of treatment courses, number of cancelled referrals, number of Did Not Attends (DNA), and waiting times for referrals and after referrals are made. Results were as follows:

Results of the first question of the audit had shown that $100 \%$ of the children included in the audit had the same referral method as being referred from a paediatrician, which follows the local guidelines for referral to the clinic. 


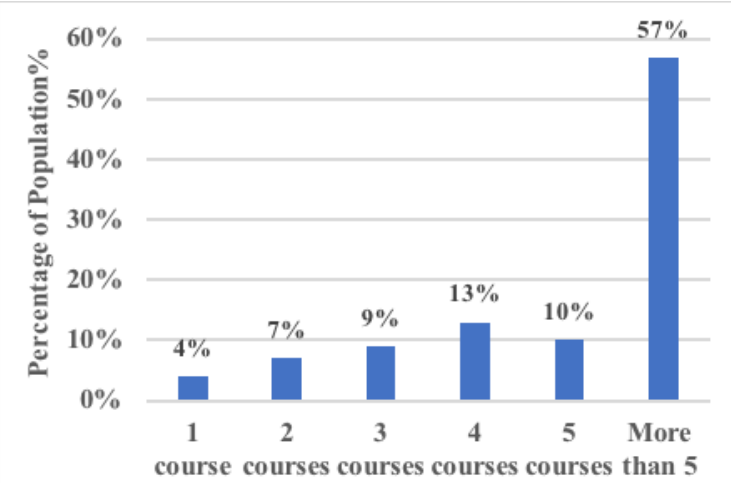

Figure 5.1 Indicating the number of previous conventional physiotherapy courses that each child had received since being first referred to the clinic.

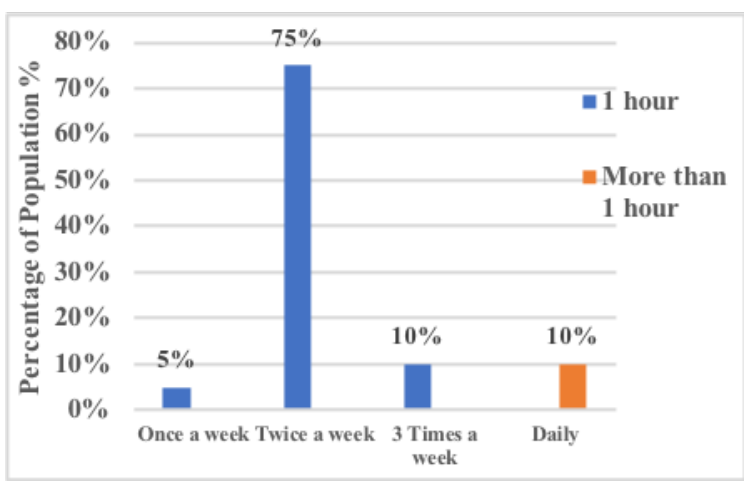

Figure 5.3 Indicating the frequency of the treatment session per week and the duration of the sessions.

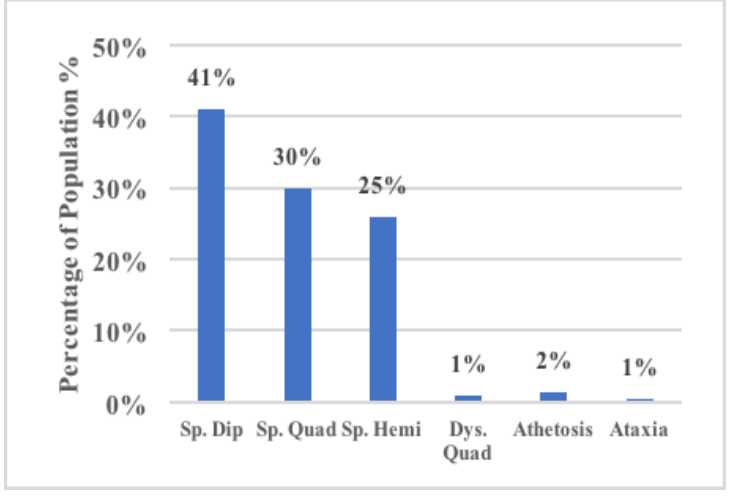

Figure 5.2 Showing the percentages of children with the different types of the cerebral palsy that are receiving treatment in the clinic

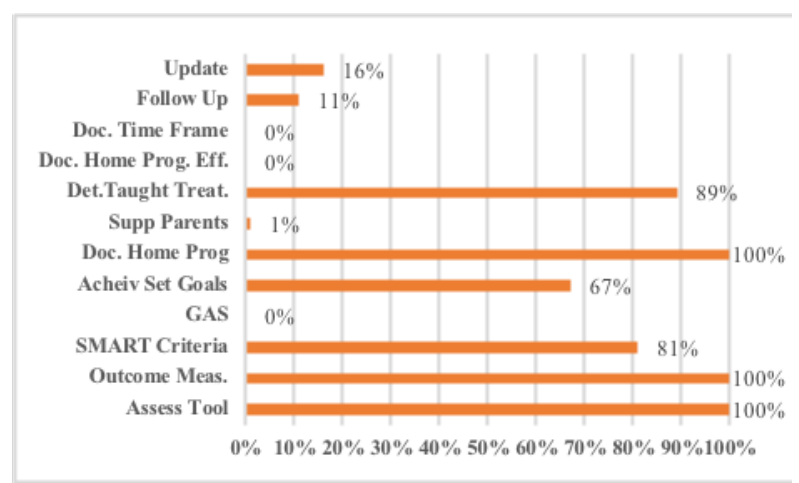

Figure 5.4 Indicating the level of documentation regarding the details of home program, goal setting and documentation, in addition to use of measurement outcome tools. 
The total percentage of the population in figure 5.1 that had received more than five courses of conventional physiotherapy treatment exceeded half the number of children included with a percentage of $57 \%$. The rest of the population showed variant numbers of conventional physiotherapy treatment courses between 1 and 4 previous courses per patient. Only 8 children, however, have had a single course of conventional physiotherapy treatment compared to the other children included. This, however, did not show any correlation with regard to age or severity of $\mathrm{CP}$ level.

Results have shown that only a total of $20 \%$ (46 patients) of the population have received intensive blocks of physiotherapy (Daily sessions of 1.5-2 hours of Bobath influenced physiotherapy treatment) compared to the other $80 \%$ (186 patients) who were included that did not receive intensive blocks of physiotherapy treatment. Out of the $20 \%$, only one patient had received three courses of intensive blocks and 7 patients which is $4 \%$ have received two intensive blocks. All the rest that represent $16 \%$ have received only 1 intensive block of physiotherapy treatment. In all the records included none of the cases has received more than three courses of intensive physiotherapy treatment.

Table 5.2 Indicating the number of patients with cancelled referrals

\begin{tabular}{|l|l|l|}
\hline & Number of patients & Percentage \\
\hline 1 Cancelled referral & 31 & 13 \\
\hline 2 Cancelled referrals & 6 & 3 \\
\hline 3 Cancelled referrals & 3 & 1 \\
\hline 4 Cancelled referrals & 1 & .4 \\
\hline 5 Cancelled referrals & 1 & .4 \\
\hline Total & 42 & 18 \\
\hline Missing Population & 190 & 82 \\
\hline
\end{tabular}


Table 5.2 shows that a percentage of $18 \%$ of the 232 patients included have had at least 1 cancelled course of physiotherapy treatment which means that after the start of the physiotherapy treatment course the child's physiotherapy treatment course had been stopped and the child had to be taken to the Out-Patient Department (OPD) to see the paediatrician again to be referred back to physiotherapy treatment. The child's physiotherapy treatment is usually cancelled due to the continued absence of the child from the physiotherapy treatment sessions.

A Did Not Attend (DNA) is a phrase that is used in the department of physiotherapy to indicate that the child is referred to the physiotherapy department by a paediatrician to receive physiotherapy treatment had not attended the first session appointed. Usually, after a child had been referred to the department by a paediatrician they are assigned to the waiting list and would be contacted to attend an initial session with a physiotherapist. If the parents and the child fail to attend that initial session the parents will be given another appointment and if the parents also fail to attend the second appointment a third appointment will be given and only after the parents have missed the third appointment that the child's referral will be considered as a did not attend and would then be removed from the waiting list and must attend back to the OPD to see the paediatrician again to be referred back to therapy. A total of $22 \%$ of the children have had at least 1 previous incident of DNA and not attending for treatment sessions after being referred which is a total number of 50 children. Furthermore, $5 \%$ have had 2 DNAs and a percent of $1 \%$ had three incidences of DNAs from the total population of 232 patients included.

A majority of $66 \%$ had shown a waiting period of two weeks or less to start the treatment sessions after being referred. On the other hand, $25 \%$ had indicated the waiting period to reach a one-month time while a minority of $3 \%$ have had to wait more than two months to receive treatment sessions.

As for the between blocks of treatment waiting times a total of $97 \%$ of the population had shown to have a response to this question of the audit, leaving a $3 \%$ that did not respond which was due to having received only a single course of treatment at the time when the data of the audit was collected. More than $44 \%$ of the responding population has shown to have waited more than two months to be referred back to the treatment sessions after their courses 
have come to an end. A little over $24 \%$ have had a shorter waiting period of up to one month compared to $18 \%$ for up to two months and only $12 \%$ that have had no waiting periods and have been receiving continuous treatment.

\subsubsection{Information regarding the diagnosis of the patients}

Section B of the Audit Collection Tool had recorded the percentage of types of CP cases referred to the clinic as well as the severity of the cases.

Figure 5.2 shows that the most common type of cerebral palsy seen in the clinic was spastic diplegia with $41 \%$ of the total population seen in the clinic. Spastic quadriplegia followed with $30 \%$ and hemiplegia with $25 \%$. Other types that are considered rare were seen in low percentages that included dystonic quadriplegia (1\%), athetosis $(2 \%)$, ataxia $(1 \%)$.

Results of the audit showed level I to be the most common level of CP on the GMFCS with a percentage of $28 \%$ followed by level II, III, V, and IV with near values of $24 \%, 21 \%$, $14 \%$, and $13 \%$ respectively.

\subsubsection{Information regarding the parameters of the treatment sessions}

The data gathered in section $\mathrm{C}$ of the Audit Collection Tool had included the parameters of the physiotherapy treatment sessions that are delivered in the clinic. Parameters had included the duration of the treatment session, and the frequency of the treatment session per week. Table 5.3 presents the findings. 
Table 5.3 Showing percentages of the different parameters used to control the intensity of the sessions of treatment provided

\begin{tabular}{|l|l|l|}
\hline \multicolumn{2}{|l|}{ Number of patients } & percentage \\
\hline \multicolumn{2}{|l|}{} & \multicolumn{2}{|l|}{} & 5.6 \\
\hline Once a week treatment & 13 & 75 \\
\hline Twice a week treatment & 174 & 9.5 \\
\hline 3 times a week treatment & 22 & 9.5 \\
\hline Daily treatment & 22 & \\
\hline & & 0 \\
\hline Treatment session less than 1 hour & 0 & 91.8 \\
\hline Treatment session 1 hour & 213 & 9.1 \\
\hline Treatment session more than 1 hour & 21 & \\
\hline
\end{tabular}

Figure 5.3 indicate that in general treatment sessions are delivered in a frequency of twice per week (75\%) with some rare cases that may receive three or more sessions per week $(10 \%, 10 \%)$ however never less than once a week $(5 \%)$, while the duration of the treatment sessions delivered in the clinic to be a total of 1 hour in in the majority of times $(91 \%)$ and may exceed a 1 hour limit in cases of intensive treatment (9\%) while the treatment sessions in the clinic are never delivered in a less than 1 hour approach.

\subsubsection{Information that includes documentation procedures}

The documentation information retrieved from the records via section D of the Audit Collection Tool had included percentages of the use assessment sheets, the tools used for recording set goals for the patients, in addition to the approach and quality in which the home program was delivered to the parents of the children included. Finally, the percentages of the use and the number of progress sheets used in the records to identify the level of progress observed by the therapist. Results are shown in table 6.4: 
Table 5.4 Showing the numbers and percentages of the records that represented the level of documentation of assessment and home program support.

\begin{tabular}{|c|c|c|c|c|}
\hline & \multicolumn{2}{|c|}{ Number of pts } & \multicolumn{2}{|c|}{ Percentage } \\
\hline & Yes & No & Yes & No \\
\hline Use of assessment tool & 232 & 0 & 100 & 0 \\
\hline Use of outcome measure & 232 & 0 & 100 & 0 \\
\hline Use of SMART principle & 188 & 44 & 81 & 19 \\
\hline Use of GAS principle & 0 & 232 & 0 & 100 \\
\hline Goal achievement & 155 & 77 & 67 & 33 \\
\hline Documentation of home program & 232 & 0 & 100 & 0 \\
\hline Support provided to the parents & 3 & 229 & 1 & 99 \\
\hline Details of taught treatment techniques & 206 & 26 & 89 & 11 \\
\hline Documentation of effect of home program & 0 & 232 & 0 & 100 \\
\hline $\begin{array}{l}\text { Documentation of time frame for home } \\
\text { program }\end{array}$ & 1 & 231 & 0.5 & 99.5 \\
\hline $\begin{array}{l}\text { Follow up of parents for application of the } \\
\text { home program }\end{array}$ & 25 & 207 & 11 & 89 \\
\hline Updating home program & 36 & 196 & 15 & 85 \\
\hline
\end{tabular}

All records included within the audit (100\%) had assessment sheets added by the therapists that were used to family history, medical history, subjective and objective clinical assessment for all cases seen in the clinic during audit time period (i.e. 2010-2013) and these sheets included an indication at least one assessment tool was used to evaluate the patient's different aspects of symptoms. 
The audit had shown that every record assessed from the total of 232 records had included an outcome measure. Eighty-one percent of therapists were using the SMART principle (Specific, Measurable, Achievable, Relevant, Time-bound) when documenting the treatment goals for the children in fig 5.4, while none of the therapists $(0 \%)$ in the clinic had used the GAS (Goal Attainment Scale) in documenting the treatment goals. As for goal achievement, $67 \%$ were reported to achieve the set goals that were documented at the beginning of the treatment course while only $33 \%$ were reported to fail at the end of the treatment course to achieve the set treatment goals.

All patient records assessed within the audit (i.e. 100\%) did indicate that a home program was documented by using a sheet that only contained picture illustrations of the techniques prescribed for delivery at home by the parents. Only $1 \%$ of these records have shown further support for the parents to deliver the home program at home as shown in fig 5.4. Although some details were found to further explain the home treatment, such as mentioning that practical demonstration and application has been provided to the parents in addition to notes under the images to provide further explanation for application in some records assembling $89 \%$.

All therapist has reported that the home program has shown an effect on the child's progress which is seen in fig 5.4, although no time frame was seen to correlate the effects of the home program with the time period in which the home program was delivered neither was there a frequency and intensity of the application of the home program.

Only $11 \%$ of the assessed patient records have shown that follow up was provided to assess the adherence of the parents to the prescribed home program suggested by their physiotherapist. All the other records ( $89 \%$ ) have failed to show any signs that parents were monitored or that efforts were made to encourage parents to apply for the home program.

As for the home program sheet, it was found that it was rarely updated throughout the treatment course and the majority of records $(85 \%)$ had only shown that one home program sheet was given to the parents to apply throughout the course of physiotherapy treatment, which may exceed in some cases two months of treatment. Although results had indicated that $90.5 \%$ of the records had more than one progress sheet that reported the progress that the child made during the treatment course also providing the changes that the therapists have made to the treatment intervention and treatment goals according to the child's prognosis. 
However, such notes and updates had not included the home program's techniques and exercises. 


\subsection{Discussion}

Clinical Audits are methods of quality assurance and clinical governance that are used to assess and evaluate ongoing practice in specific sites of services (Vanu Som, 2004). They aim to evaluate existing practice in order to improve aspects that show lack of efficiency compared to best practice with regard quality integrity, the process of care in addition to the outcomes of care (Benjamin, 2008). According to the results of the audits practice is changed and improved to achieve higher standards of services and audits are then repeated to reevaluate the level of services again after the change (Benjamin, 2008). In this study, a clinical audit was performed in a paediatric physiotherapy department in the Physical Medicine and Rehabilitation Hospital (PMR) in Kuwait. The audit was performed to evaluate the services provided by the clinic to children with cerebral palsy. It had focused on the type of the courses provided for the children with $\mathrm{CP}$ and the criteria of referrals, waiting times and attendance monitoring. The audit had also assessed the types of $\mathrm{CP}$ and the levels of severity that are provided care in the centre. The intervention sessions were a part of the evaluation that included the duration and frequency. Documentation and achievement of treatment goals were also part of the audit. Another important aspect had been the home program. Assessment of the home program had included the method of education, the method of documentation, the level of support and follow up that is provided for the parents. Amount of follow up for the promotion of adherence in addition to the time frame provided for each group of management techniques were evaluated. Finally, the level of update of the existing home program was evaluated according to the effects on the child's progression. The evaluation had also included the documentation of progression levels in therapist's notes.

\subsubsection{The Nature and Intensity of the Provided Physiotherapy Course for Children with $\mathbf{C P}$}

The audit had initially shown that the criterion by which the children were generally referred to the clinic via a paediatrician only. No other methods were found in the clinic in which the children were referred by. No walk-in services were available in the centre for parents and caregivers of children that were suspected to have some abnormalities noticed by parents or caregivers. The centre also did not accept any referrals made by GPs or Nurses. The physiotherapy services in the centre and in Kuwait general is only provided to patients that have already been investigated by a specialty physician who then makes the decision 
regarding the need for physiotherapy services. This was evaluated to learn about the procedures that the families needed to go through in order to finally receive physiotherapy services. This was to evaluate the duration between the early detection of symptoms of the child and the time in which physiotherapy treatment had started. Understanding the procedures and duration helps to understand if it was easy to get access to physiotherapy services and if not has it been a burden to go through procedures which may indicate the level in which parent would cope with further procedures in the future to continue with the physiotherapy services and if the quality of services received was worth the efforts especially when results have been seen on the children's prognosis. Unfortunately, the scenario of being seen by a Physician prior to being seen by a physiotherapist only affects the patients and families by increasing the waiting times that the families have to endure before starting their physiotherapy sessions. Given the nature of $\mathrm{CP}$, a continuous approach of physiotherapy management should be provided for children.

The services were also evaluated for the number of treatment courses each child had received to understand how long has the patient been receiving treatment. This information reflects the overall period of time that patients receive physiotherapy services since being first referred until they are finally discharged from physiotherapy services in the clinic to be managed either at special schools with physiotherapy services or at home with full equipment of home handling provided.

The services provided in the centre have shown to be of great variety in intensity and was shown to be similar to conventional care and intensive care that had been identified in the literature. The audit had also indicated that children were receiving treatment regularly even though in an approach of 2-3 month blocks rather than continuous treatment. Even though some children have only received a single block of treatment, the reason in which they have only received one block was not further investigated. However, it could have been that the children were young and were recently referred to the centre and haven't yet had the chance or time to be referred to treatment more than once. Other factors may be that the children were referred to another centre or have been away for treatment abroad or even have left the country due to not being citizens in Kuwait. In general, percentages show that the majority of children of the population that have been receiving treatment have had minor waiting periods of time between blocks of treatment which indicates the intensity of the treatment courses delivered in the centre. However, the remaining minority are still facing some prolonged waiting time which is affecting the outcomes of the children and the 
maintenance of the achieved goals. Another reason for the audit showing single courses for some children would be that the children have been receiving intensive therapy rather than conventional treatment.

As for intensive blocks of treatment, children in the centre are referred to intensive therapy due to more than one reason. Children may be referred to receive intensive therapy after undergoing musculoskeletal surgery of tendon release, dislocation reduction, or malformation corrections that require post-op intensive rehabilitation interventions. In addition, children are referred to intensive therapy after receiving Botox treatment or casting to achieve maximal results. Children that are younger (1-4years) and are responding well to treatment and are showing good prognosis are known to be in an active stage of age and are also referred to intensive treatment to achieve maximal results. These values reflect the availability of the intensive intervention however it also reflects that only a small number of children are receiving intensive treatment compared to the total population. Further investigations may be established in the future regarding if all the children that are in need of intensive treatment are actually receiving the intensive approach, as this study did not focus on this aspect.

\subsubsection{Policy of Referrals and Absence}

The number of cancelled referrals and DNAs had reflected the level of commitment of the families to the physiotherapy sessions. This had helped in expecting the level of overall commitment of families under physiotherapy treatment in PMR hospital which had helped in predicting the level of commitment of families to the pilot RCT that was planned to be implemented. The waiting periods between physiotherapy courses and waiting times to start physiotherapy courses after referrals were evaluated to acknowledge the duration in which the families were expected to wait for physiotherapy services. These periods were expected to have an effect on the attitudes of the families toward the value of physiotherapy sessions and if it was easy for them to get access to physiotherapy sessions even if they were not committed to attend and have had their physiotherapy courses cancelled previously due to poor attendance and commitment.

The records have shown many cases of the referrals of the children being cancelled or labelled as Did Not Attend (DNA). Children that fail to show at the beginning of the treatment block have their referral sheets marked as DNA and are then expected to get a new 
referral if they wish to continue to receive treatment. In other cases, that children miss three sessions in a row during the treatment course they also have their courses stopped and they are again expected to get a new referral to a new treatment course. After investigating the following aspect of the audit the high number of cancellations and DNA were found to be justified to a limit. While the children are receiving their treatment courses and are approaching the end of the treatment the system in the clinic allows the parents/caregivers to set an appointment to see the paediatrician after the end of the block. It was found from the audit that the waiting time to see the paediatrician following the treatment course or after the treatment course has been cancelled may extend up to six weeks before they are seen. Although as soon as they are examined by the paediatrician and referred back to treatment the waiting period to begin the sessions were more acceptable. From the author's experience, it was found that parents/caregivers did not find the waiting times to be unacceptable and therefore were found to be less adherent and punctual with the attendance at their sessions. Also from the years of experience of the author, it was found that children are always referred back to treatment courses in the centre even if no commitment was found to the courses of treatment. This affects the children's level of developmental change, therefore, the department should encourage more appreciation and commitment to physiotherapy sessions that may be induced by raising awareness whether regarding the nature of $\mathrm{CP}$ or regarding the importance of physiotherapy for children with CP.

\subsubsection{Cases Receiving Physiotherapy Services in the Department}

The numbers of types and severity of cerebral palsy cases were assessed to identify the variety of cases and severities that were attending the paediatric department in PMR hospital to be later compared to the percentages identified in other studies of the literature. This information had also helped in supporting the types of cases required for the feasibility of the pilot study that was applied later on in this research (Chapter six).

\subsubsection{Physiotherapy Treatment Intensities}

The parameters that were assessed in the clinical audit was to establish a comparison between the physiotherapy service intensities in PMR in Kuwait with other paediatric physiotherapy intensities provided and evaluated by other studies in the literature. The 
intensities were also assessed to support the methodology of the pilot clinical trial applied in the next chapter.

It was found from the audit that they are mainly divided into the frequency of the sessions per week and the duration of the treatment session itself which is in accordance to what was referred to as session intensities in the literature (Adams et al., 2000; Knox \& Evans, 2002) that had reported the treatment sessions to be similar to what is being delivered in the department of paediatric physiotherapy in PMR hospital Kuwait of two to three sessions per week. In the centre, the sessions are delivered in the conventional blocks of treatment two to three times a week. At the time when the audit had taken place, evaluation was done on the latest treatment course found in the patients' records.

From the researchers own clinical experience it was found that overall the sessions in the centre are delivered in 1-hour duration of conventional physiotherapy treatment. Children that are receiving conventional therapy receive a 1-hour hands-on treatment session. In some cases, that require positioning as an intervention the 1-hour session is then followed by a 30minute positioning session on a flexible or prone stand. However, the positioning session was found to be mentioned in the plan of the treatment with rarely any further details mentioned in the records regarding the duration or type of stand used. Very few records had documented the details of positioning although most of the treatment plans have had positioning mentioned as part of the plan. Other than that, in the intensive treatment courses, the session consists of 90 min hands-on treatment followed by a positioning session if applicable to the case. None of the records assessed has shown patients receiving a treatment session of less than 1 hour. Indicating a sufficient duration of the treatment session and in compliance with the literature (Adams et al., 2000; Bar-Haim et al., 2006; Tsorlakis et al., 2004) especially when providing treatment for children. Children require longer duration in order to understand and perform the treatment techniques as required by the therapist compared to adults. In addition, children may in some conditions be difficult to keep focused and interested in the treatment session, therefore, the duration of the treatment session plays a role in indicating the quality of the treatment provided. These findings would be of value in designing the methodology of the following chapter that includes a pilot trial.

The treatment methodologies on the other hand that include the treatment intervention techniques and approaches similar to other studies in the literature were not clearly documented in the patients' records. The records had only stated general information such as 
NDT treatment, home treatment, or positioning, or strengthening with no further details stated.

\subsubsection{Validity and Reliability of Outcome Measures}

When treatment outcomes were evaluated results have shown $66.8 \%$ of set goals have been achieved indicating that more than $30 \%$ of set goals have failed to be achieved. Therefore, in an effort to further understand the results of this aspect the validity and reliability of the outcome measures have been evaluated especially when the records have failed to report the details of the interventions provided which made it impossible to evaluate the validity of the treatment methods.

The outcome measures were evaluated in order to assess the validity of assessing the level of change that has been detected as a response to the treatment intervention provided. In relation to the level of goal achievement that had been assessed, this had helped in identifying if the level of goal achievement was due to lack of validity of outcome measures used to detect a change in child's prognosis or due to a lack of validity in the physiotherapy intervention provided for each child. This issue must be addressed by the department of paediatric physiotherapy in order to improve the level of progress achieved. This would also help select a valid outcome measure to be used in the pilot RCT in the following chapter.

In the audit section assessing the documentation criteria of the assessment tools, treatment plan, home treatment, and progress follow up, the audit results had shown an acceptable level of documentation given that almost all aspects that had been investigated by audit had been detected and identified from the patients' records. As there were no aspects of the investigation in the audit data collection sheet that had been left incomplete due to an insufficient amount of information in the patient's records that had been documented by physiotherapists.

Another aspect of the assessment was the use of at least one assessment tool whether to measure specific outcomes such as range of motion (ROM), spasticity, or gross motor functions. Audit results were found to indicate the use of at least one assessment tool in all $232(100 \%)$ records included. Indicating that objective measurements were well recruited in the assessment of children in the centre. 
As for treatment plan for the children, it was found that the majority of records in the documentation of treatment goals have used the SMART principle to indicate specific and measurable goals. The SMART principle indicates the initials of the main aspects that a treatment goal must include to be valid and including the aspects of being: Specific, Measurable, Attainable, Realistic, Time-framed. Using this principle in more than $80 \%$ of the records evaluated indicates the commitment to obtaining the set goals for each child. However, the "measurable" aspect of the SMART principle was found to be missing from the documentation. There were some efforts found to measure changes functionally that were not found to be reliable or valid. These findings were considered gaps that were found in practice that may have affected reaching the goals set for patients.

The Goal Attainment Scale (GAS) which is one of the most reliable and valid measures of change in patient's abilities (Hurn et al., 2006; Mannion et al., 2010) was found not to be used at all in any of the evaluated records. Showing that it is not considered to be a popular instrument for goal setting and achievement in Kuwait even though compared to Kiresuk et al. (2014) it is considered widely used as one of the most valid and reliable method of outcome measures and to Steenbeek et al. (2011) in some situations even more reliable and valid than gross Motor Function Measure 66 and the Paediatric Evaluation of Disability Inventory in detecting change. None of these outcome measures was found to be documented in the evaluated records. Therefore, this was also considered to be a gap in practice.

Although the SMART principle is a valid tool for goal setting (Siegert \& Levack, 2014) it may not be effective in measuring the level of achievement of those goals such as the GAS (Siegert \& Levack, 2014). Therefore, it could be argued that even though the audit has shown a high percentage level of documenting SMART goals in the centre the same efforts were not put into achieving such SMART goals and measuring the levels of achievement of these goals and the level of change identified in a child's functional level as objectively as when employing the GAS. The audit had shown acceptable levels (66.8\%) of goal achievement, however, the inability to objectively measure the attained goals may affect the validity of achievement (Hurn et al., 2006; Siegert \& Levack, 2014). In many cases using the GAS may predict minor changes and improvements that may not be obvious in cases where the GAS is not used and other gross motor functions are set as goals which in this case may indicate the failure of achieving set goals especially with children showing slow progress. 
In the section assessing the home program, the audit had indicated that all the records assessed had included a home program sheet indicating the overall policy of providing a documented home program for the parents to apply at home in the frequency recommended by the therapist. However, the documentation did not reflect a complete intervention approach in handling the child at home. The documentation had only revealed a sheet of illustrations of the positions, simple exercises and handling techniques, or corrections of incorrect handling. This does not reflect the level of support that should be provided for parents in handling the children at home. Especially when the children spend their time mostly with parents and caregivers compared to the very limited time of 2 hours a week on average receiving physiotherapy. Therefore, a gap was detected in the delivery, education and follow up of home management.

Support to the parents may take place as an educational session, regarding the importance and effects of the home program. It may also be provided as a practical training session to the exercises and handling techniques, by dedicating a treatment session to practice and train the caregivers for providing the exercises and handling techniques that are provided in the sheet. To ensure the understanding and the correct delivery of the exercises required by the therapist. In addition, the therapist may share the experience of applying the treatment at home and the discussion of the difficulties or obstacle that may have emerged from the experience of applying the treatment at home to better support the caregivers in providing the home treatment. Even though such support was being provided subjectively or practically during the session only $1 \%$ was found to document such support in the patients' records. Furthermore, no documentation was found regarding the changes that may have occurred due to the application of the home program. No documentation was found either to report the changes experienced when applying for the home program by the parents/caregivers such as a specific exercise becoming easier and less challenging for the child. Reporting changes seen in the child's motor abilities in the home environment is considered an important indicator of changes in the child's prognosis whether due to the clinical intervention or the home program (Halvarsson et al., 2010; Whittingham et al., 2011). Therefore, the lack of any information regarding the changes in ease of application of home exercises indicates a lack in reporting part of the prognosis of the child.

Lack of information regarding the update of the home treatment sheet or a time frame was detected from the records which is considered to provide a better quality of the level of home treatment in addition to reflecting a better level of follow up provided by the therapists 
which according to (Basaran et al., 2014) promotes higher levels of adherence to the home program and therefore higher level of change in the child's prognosis. This may hinder further development of the child's functional level if not affect the maintenance of the goals that have been achieved during the treatment course.

On the other hand, the chart documentation had been evaluated for the number of progress reports present in each child's record in order to assess the frequency of progress documentation and how it may have reflected the reporting of a change in each child's symptoms. The evaluation of the overall management system of children with cerebral palsy was essential in supporting the methodology of the following chapter.

Progress notes are notes written by the therapists to identify any changes and updates seen in the prognosis of the child. In the records, more than $90 \%$ of the cases had included more than one progress note throughout the treatment course indicating the close monitoring and re-assessment provided for the patients. 


\subsection{Conclusion}

The audit had included 232 records of patients with $\mathrm{CP}$ that have been receiving treatment in the PMR hospital in Kuwait. The patients' records included have been from the years 2010 to 2013 . The results of the audit had revealed gaps in practice in more than one aspect. The gaps have reflected prolonged waiting durations for physiotherapy services that may reach up to 8 weeks in some cases that may interrupt with the continuous principle of physiotherapy intervention for children with CP. The level of adherence to the provided physiotherapy courses have been found to be in moderate levels with at least one cancelled physiotherapy course due to absence was detected for each child. Other limitations were found in the method used for documentation of treatment goals that may have resulted in the lack of objective measurement of change in the child's condition as a response to physiotherapy. This had led to decreased percentages of goal achievement. As for the home management, the audit had revealed limitations in the educational support to parents regarding the management and nature of CP. In addition, some limitations were identified in the methods of delivering the home treatment and follow up on the application of treatment at home. The effects and feedback of home treatment were also found not to be assessed or documented in the patient records. As these aspects of improper practice have been identified by the clinical audit were intended to be changed and corrected. Therefore, according to Benjamin (2008) and the NHS guide to clinical audit changes must be implemented in practice and evaluated for further effectiveness in improving services. A pilot trial, therefore, planned to apply the changes into service in order to investigate the level of effect.

\subsubsection{Recommendations for Service development}

One of the functions of an audit is to describe current practice within a clinical service in order to identify potential approaches that could be implemented to improve effectiveness and/or efficiencies that would increase patient satisfaction and increased the quality of care being provided. As indicated by the findings of this audit there are some recommendations that could be generated that may improve the current services: 
- Provide a measurement tool such as GAS or other instruments for achieving functional goals that have been stated.

- Provide to parents who will be expected to deliver a home program a training event that would give them a practical demonstration and an opportunity to practice the application of the prescribed exercises. This training event would also allow for the importance of each exercise and the expected effect of providing it to be highlighted.

- Ensure that all documentation relating the delivery of the prescribed home program records the observed (subjective) and measured (objective) effects.

- Home Program information provides to parents should include key details about the time frame for the application of specific home program exercises (i.e. 1 hour) and information about what the expected observed effects would be.

- Ensure that parents who have been prescribed a Home Program are followed up to determine if they are applying for the home program and to check if there are any observed changes in the progression of their child.

\subsubsection{Limitations}

The limitations of the clinical audit had included:

- Missing data that has been not found in the records that are usually documented as indicated by the department's documentation policy.

- Poor handwriting in some situation that affected the understanding of the information documented.

- Patient records were missing from the fire department due to being at the ward or at the outpatient clinic. 
- The geographic area that was covered in this audit that included only patient records at the PMR hospital may not have presented the total population of children with $\mathrm{CP}$ in Kuwait.

The results of this clinical audit had identified some aspects of practice that may require further development in order to provide higher levels of service qualities and therefore had fulfilled the objectives of the audit. These results would be further used to provide some improvements of the services to evaluate the level of effect that these changes have had in improving the quality of the services provided in a pilot experimental trial. 


\section{Chapter 6}

\section{Does Providing Parents Additional Support for The Delivery of a Home Program Improve the Functional Outcomes of Bobath? A Pilot Trial Study.}

\subsection{Introduction}

In the paediatric department in The Physical Medicine and Rehabilitation hospital (PMR) in Kuwait where the Clinical audit in chapter five has been conducted, the main intervention used for children with CP is either Bobath or a Bobath influenced approach. This may also be combined with other interventions to support its outcomes similar to functional therapy, strengthening and/or stretching. This depends on the physiotherapist experience and qualification.

The clinical audit study, detailed in chapter five, had not evaluated the type of intervention provided, however, it had indicated that structured support that must be provided to families was inconsistent. This principle is not being utilized to its full potential by providing structured support for parents to continue with the prescribed procedures of home treatment in the paediatric department in PMR hospital in Kuwait.

As outlined in chapter 1, Bobath is a widely used approach in Neuro-rehabilitation of various neuro-deficits including cerebral palsy (Marret et al., 2013). Since it was developed by the Bobaths in the 1940s the approach has offered a problem-solving framework to the therapists working with motor deficits and Upper Motor Neuron (UMN) and Lower Motor Neuron (LMN) lesions by providing a specially tailored treatment for each patient, which includes exercises that can be delivered by parents at home to ensure continuity of treatment.

The approach provides management for the child as a whole for both severe and mild motor deficits. The general aim of the Bobath treatment is to provide the patient with control over stability and alignment in addition to encouraging more efficient movement patterns with minimal assistance attainable (Graham et al., 2009). Control is then influenced by creating an appropriate length of muscle to achieve functional movement components through sufficient joint range (Graham et al., 2009). To influence the achievement of the complex relationship between stability and mobility selective strength training exercises using body weight against gravity are used during functional activities (Graham et al., 2009). 
Interventions (such as Bobath) that are used for the management of symptoms in children with cerebral palsy require effort, practice, and continuous delivery to achieve benefits. Therefore, input from parents and caregivers is essential (DeGangi \& Royeen, 1994; Raine, 2007). As the child with cerebral palsy receives only a limited duration (two to three hours) of weekly treatment in the clinic it is essential to take advantage of the period between treatments treatment by providing treatment at home. The therapist should, therefore, work with the family and/or caregivers to educate them regarding the handling, treating and positioning of the child at home, which will encourage and facilitate correct movement.

As outlined and discussed in the literature review (chapters two and three) various clinical parameters, treatment/management options and physiotherapeutic techniques could be used as part of the overall clinical management of cerebral palsy; these treatment options include various home-based programs that aim to maintain or improve the efficiency of the therapist led interventions. Different levels of evidence were also investigated in chapter four. The evidence from the literature was evaluated using the CASP principle and only articles that were rated a score above eight were included in the review. The aim was to gather the best evidence to support the application of home management by the parents. The evidence of best practice for rehabilitation of children with cerebral palsy and home program delivery was found to be inconclusive in the literature and requires further research. Some approaches, however, such as the Bobath approach are more popular than others.

\subsection{Aim of pilot study}

To explore the impact of supporting parents in providing home treatment aimed at improving functional development through Bobath Therapy.

\subsection{Study Objectives}

This study applied an experimental trial (as described and evaluated in chapter 4) designed to assess the following objectives:

- To evaluate the physiotherapy services provided to the children prior to taking part in the trial

- Assess the response of parents and caregivers to the trial. 
- Assess the views of parents regarding the physiotherapy services provided.

- Perform a feasibility study to investigate the applicability of the pilot RCT.

- Asses the effect of the educational course on elevating awareness level and level of adherence to the home treatment to support clinical physiotherapy to promote goal achievement.

- Assess the effectiveness of the interventions in improving outcomes and overcoming limitations identified from the audit.

- Assess the level of adherence of caregivers to the home program.

- Assess the effectiveness of a new encouragement and follow up method provided by the researcher to those following the home program as a weekly home visit.

- Assess the feasibility of developing the pilot study into a larger scale randomized controlled trial.

\subsection{Piloting of Methodological Approaches}

A feasibility study was performed prior to the pilot study to identify any difficulties or obstacles that may require changes to any part of the trial.

The feasibility study included six children with $\mathrm{CP}$, divided into three groups.

As a consequence of parent's concern for their child's benefit of treatment the 8 weeks "off treatment period", which is the period in which the child is not receiving any clinical physiotherapy, was reduced to only six weeks. The educational course (Appendix 11) was provided to the parents in group B (Educational Group) and C (Home Visit Group) together which had led to the parents exchanging details of the trials stages with each other. This caused some families in the educational group to ask for the home visit that was offered to the participants of group C (Home Visit Group). To avoid this conflict, the educational lecture was then given to each group in a different timing. The educational lecture had been provided for parents early in the feasibility study following the baseline assessment. However, a better outcome was expected in the pilot study if the lecture was provided immediately before the "off period". 


\subsubsection{Impact on Study Design}

- The "Off period" was reduced to six weeks.

- The educational lecture is to be provided to groups B and C separately.

- The educational lecture is provided immediately before the "Off period' rather than after baseline measures.

\subsection{Pilot Study}

\subsubsection{Experimental Design}

A pilot randomized control trial design was used to investigate the objectives set for the trial. The design was selected as the most suitable method according to the literature (Adams et al., 2000; Knox \& Evans, 2000; Tsorlakis et al, 2004).

\subsubsection{Methodology}

\subsubsection{Participant Sample}

This pilot study is to assess the effectiveness of a specific intervention of Bobath treatment for children with $\mathrm{CP}$ with specific parameters. Accordingly, the sample size was chosen to be the minimum number that would provide meaningful results, which was deemed to be 10-15 for each group of patients receiving different parameters of treatment (Hertzog, 2008). The study had initially contained 33 children with cerebral palsy between the ages 1 and five. The severity of the children's disability included levels I, II, and III on the Gross Motor Function Classification system (GMFCS) with all children having the ability of locomotion. The parents of the children were invited to participate in the study by the physiotherapists responsible for delivering their current course of physiotherapy. If any of their children fulfilled the inclusion criteria for the study the physiotherapist would inform the parents of the trial and ask if they were interested in taking part in the study. Those expressing an interest were then introduced to the researcher.

The inclusion criteria of the pilot study were: 
- Diagnosed with cerebral palsy by a consultant paediatrician, as confirmed in the corresponding referral letter.

- Between the age of one and five years.

- Classified as being between levels I and III on the GMFCS.

- Having received at least 1 course of physiotherapy treatment.

Participants were then handed a participant information sheet to outline the purpose of the pilot study and provide the full information of the pilot study. They were later asked to sign an informed consent for themselves and their children.

The exclusion criteria for the pilot study were

- The child had received a surgical or medical intervention such as Botox in the last 12 months.

- The child had uncontrolled epilepsy

- The child is receiving physiotherapy treatment for the first time. According to many studies (Franki et al., 2014; Law et al., 2011; Levitt, 2004) children with cerebral palsy tend to show slightly higher and stronger peaks of progress after receiving their first course of physiotherapy treatment compared to the following courses or would show less accelerated progress after about three months of continuous treatment.

- Patients who missed more than three treatment sessions in a row, they would be excluded from the final data analysis.

\subsubsection{Consent Procedures}

The children were nominated by their treating physiotherapist if they fulfilled the inclusion criteria and parents/caregivers were then introduced to the researcher after showing interest in being part of the study. After verbally explaining the procedures the parents were given an information sheet (Appendix 7) that included all the information and details of the trial. After deciding to take part in the study the parents were asked to sign a consent form (Appendix 8) confirming they have fully understood the trial, what treatment would be provided and what was expected from them. They were also asked to confirm that they were given enough time to discuss the study, and ask questions and freely decide if they wished to participate in the study. The consent form also explained that they had complete freedom to 
withdraw from the study at any time without any questions being asked and without this action affecting by any means their rights in receiving their treatment in the centre.

After signing the consent form the parents were then asked to complete a service evaluation questionnaire (Appendix 9) and a home program questionnaire (Appendix 10) to investigate the nature of the services that had already been received since being referred to the department and to collect their opinions of these services. The information gathered from this questionnaire was used as a secondary outcome for the trial.

The 33 children in the study were assigned to a list in the order in which they were invited to the study and were then manually assigned to the groups (A, B and C) three at a time.

\subsubsection{Trial Protocol}

The trial protocol required three groups. An observational group (Group A) which was considered the control group and 2 experimental groups (B and C). The first experimental group (Group B) received an educational course while the second group (Group C) received an educational course and a weekly home visit by the researcher. An evaluation of the level of motor function was performed at the beginning of the trial using the Gross Motor Function Measure (GMFM) for participants in all three groups. This measurement was used as a baseline for all participants. The assessments were made by two therapists, trained in the use of the GMFM, who were blinded to the objectives of the study as well as the group of which the patients were allocated to. Each of the assessors had more than 10 years of experience in the field of paediatric physiotherapy.

The children in all three groups continued to receive treatment from their usual therapists, who were also blinded to group allocation, to ensure continuity of (conventional provided) care. The decision to deliver the intervention through the child's own therapist was made to avoid the adaptation time that a child might need to adapt to a new therapist which may have affected the timeline of the pilot study and the total number of physiotherapy sessions planned for each child during the trial. This decision was in agreement with the procedures recommended by Tsorlakis et al. (2004). The adaptation duration may have biased the comparison of effects of physiotherapy intensity between children in the same group and between children in different groups. Providing the physiotherapy sessions by one or two physiotherapists generally may have promoted a higher level of homogeneity in the 
intervention provided. However, under the Bobath approach, the treatment given to each child might vary significantly (Butler \& Darrah, 2001). In the literature some studies have provided treatment by multiple therapists (Adams et al., 2000; Dalvand et al, 2009; Knox \& Evans, 2002; Tsorlakis et al., 2004; Shamsoddini, 2010) while in other studies treatment was delivered by the same therapist (Embry et al., 1990, Fetters and Kluzik, 1996). All the children in this pilot study have received a Bobath influenced intervention that consisted of a 1-hour session twice a week.

Children allocated to the groups received a Bobath influenced intervention (conventional therapy) consisting of 1-hour long session twice a week for six weeks, delivered by a Bobath trained physiotherapist. The Bobath approach is the intervention used in this research because it is the intervention used in PMR hospital in Kuwait, therefore this research was aimed to identify and develop the methods needed to improve the level of Bobath's outcomes rather than changing the intervention itself which is more realistic as an objective and more feasible. The normal routine provision of Bobath therapy within the department that the trial was being conducted in was 8 weeks of treatment aligned to the recommended principles of delivering Bobath to children with cerebral palsy (Butler \& Darrah, 2001).

However, the methodology in this study was intended to provide the same number of weeks of experimental treatment as the number of weeks of no treatment (Off Period). The period of no clinical hands-on physiotherapist treatment was reduced to six weeks in accordance with the participants request due to the refusal of parents of intentionally being off clinical treatment for over six weeks. Therefore, the clinical physiotherapy hands-on period was also reduced to six weeks.

\subsection{Group A (Observational Group)}

This was the control group of the trial. However, as it is ethically not acceptable for the children not to receive any treatment, all the children in this group received their routine treatment following the principles of Bobath (as outlined in chapter one). Functional assessments were taken at baseline (as described above), repeated after the completion of six weeks of physiotherapist led treatment (as described above), and finally repeated again at the end of six weeks off period. 


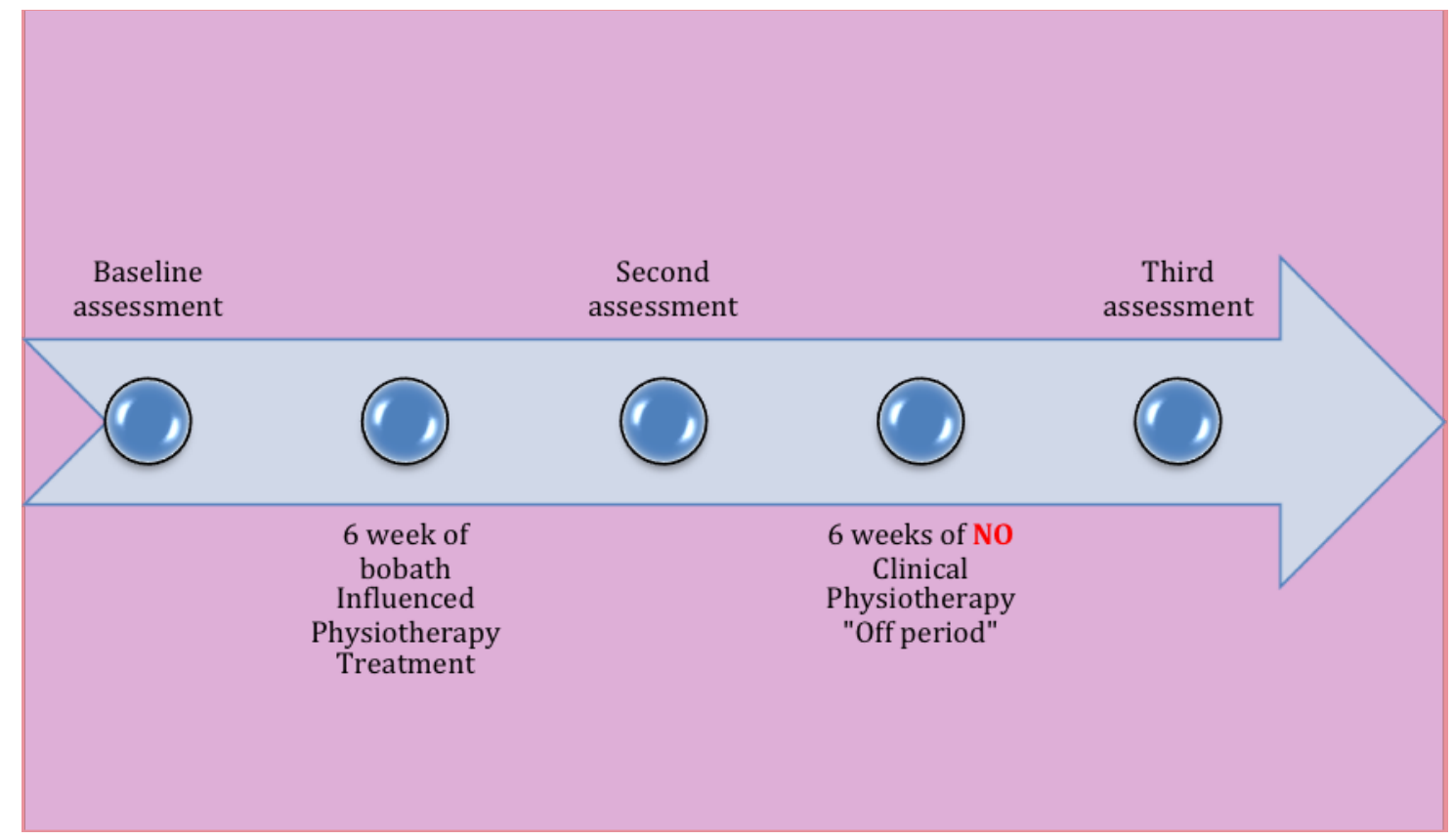

Figure 6.1 The procedures performed for group A (Observational Group) during the trial.

\subsection{Group B (Educational Course Group)}

Parents of children allocated to group B received a two-day educational course after the second assessment as a new method in Kuwait delivered as a standardized educational public lecture that provided important information that had included all members in group B. which focused on the nature, outcomes and prognosis of the disorder. The structure of this course is similar to that provided in the study of Ahl et al (2003). The lecture also discussed the importance and effect of the home program in supporting the progress of the child's outcomes. After the first day's lecture, the parents/caregivers were given a chance to ask questions regarding their children's cases. The second-day lecture comprised a one to one discussion that focused on each child's personal outcomes and a discussion of the practical application of the home program designed by the treating therapist. The parents were then expected to apply the home treatment, rehearsed during the educational course and supported by the researcher during the educational course and supported by the researcher during the six weeks off period. The final assessment was then made at the end of the six weeks off period (fig 6.2). 


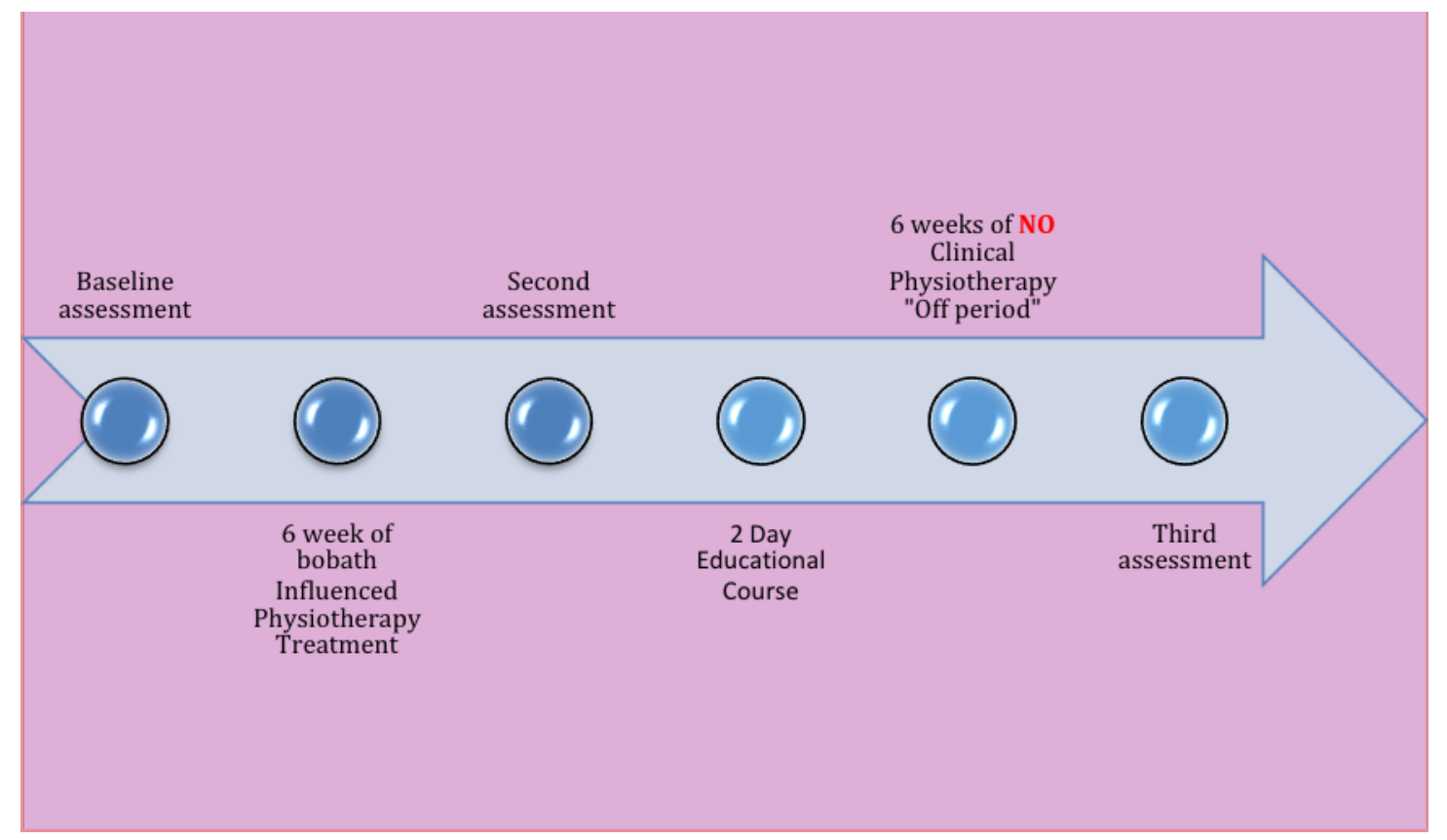

Figure 6.2 The procedures performed for children in group B (Educational Group) in the trial.

\subsection{Group C (Home Visit Group)}

These children were at baseline, and again at the end of the six weeks of physiotherapist led care (fig 6.4). Children allocated to group $\mathrm{C}$ also received as a new method of education in Kuwait delivered as a standardized educational public lecture that provided important information that had included all members in group $\mathrm{C}$ similar to the educational training lecture delivered to group B (Educational Group), but on a separate day to ensure participants did not have the opportunity to discuss their treatment allocation or experiences. The families also received a new method of follow up that consisted of a single weekly visit for the entire six -week period by a physiotherapist, at a time that was suitable for them. During each home visit, the importance of the parent-led home program of treatment (as prescribed by the physiotherapist who had responsibility of treating their child) was emphasized, encouragement was given for the parents to adhere to the application of the prescribed home program, and an opportunity was provided for the parents to seek additional advice and any questions they have had about the actual techniques they were delivering to their child. The home visit is considered to be a new method of follow up that was not found to be applied in previous research in Kuwait or other countries. On the completion of this six -week period of home visits during the "off period", a final assessment was completed. 


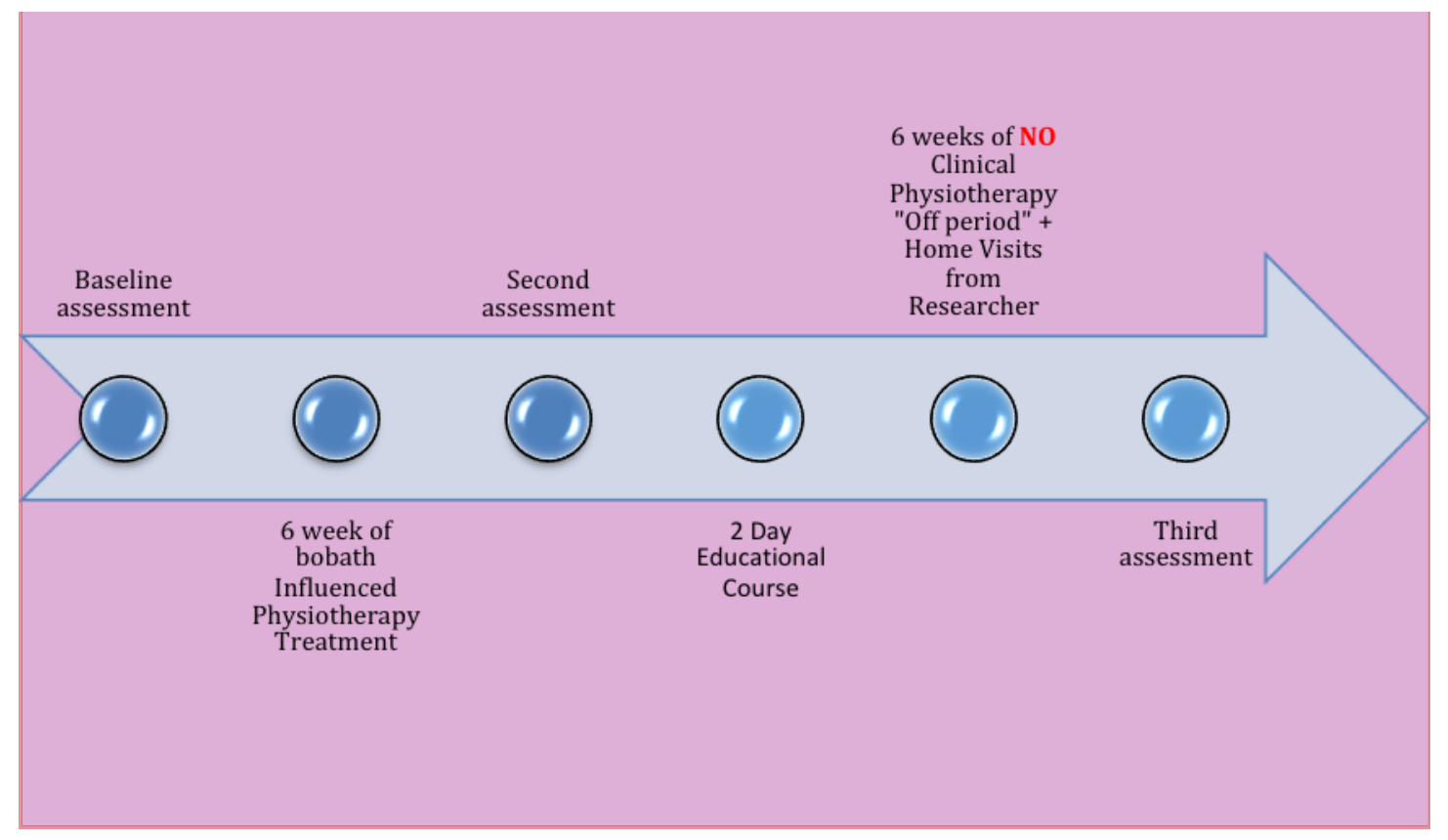

Fig 6.3 The procedures performed for children in group C (Home Visit Group) in the trial.

Table 6.1 Showing group allocation and parameters of treatment and support provided for each group.

\begin{tabular}{|c|c|c|c|}
\cline { 2 - 4 } \multicolumn{1}{c|}{} & Group A & Group B & Group C \\
\hline $\begin{array}{c}\text { Educational } \\
\text { course }\end{array}$ & No & $\begin{array}{c}\text { Educational Course } \\
\text { Group }\end{array}$ & Home Visit Group \\
\hline $\begin{array}{c}\text { Treatment } \\
\text { Regime }\end{array}$ & $\begin{array}{c}\text { The normal regime for that } \\
\text { clinical service } \\
\text { (observational) }\end{array}$ & $\begin{array}{c}\text { A Bobath influenced } \\
\text { intervention }\end{array}$ & $\begin{array}{c}\text { A Bobath influenced } \\
\text { intervention }\end{array}$ \\
\hline $\begin{array}{c}\text { Parental } \\
\text { support and } \\
\text { advice }\end{array}$ & $\begin{array}{c}\text { No dedicated support or } \\
\text { advice provided }\end{array}$ & $\begin{array}{c}\text { No dedicated support or } \\
\text { advice provided }\end{array}$ & $\begin{array}{c}\text { Dedicated support and } \\
\text { advice }\end{array}$ \\
\hline $\begin{array}{c}\text { During off } \\
\text { period }\end{array}$ & $\begin{array}{c}\text { Non emphasized home } \\
\text { treatment }\end{array}$ & $\begin{array}{c}\text { Application of the parents } \\
\text { led home program }\end{array}$ & $\begin{array}{c}\text { Application of the parents } \\
\text { led home program }+ \\
\text { Support and advice }\end{array}$ \\
\hline
\end{tabular}




\subsubsection{Primary Outcome Measure}

\subsection{Gross Motor Functional Measure}

The method chosen to evaluate the functional motor ability of the children with $\mathrm{CP}$ included in the study is Gross Motor Functional Measure (GMFM).

\subsubsection{Secondary Outcome Measure}

\subsection{Parents Evaluation of Previous Service Questionnaire}

It was essential to understand the parents' views and opinions of the physiotherapy service they had previously received prior to taking part in the study. The views were expected to have an effect on their attitude toward this study and the home treatment taught and supervised in addition to the adherence to the application of home treatment.

The questionnaire was initially developed according to the researcher's base of professional experience in clinical services. The initial format of the questionnaire was refined through discussion with colleagues to improve and develop the depth of the questions and enhance the layout and formatting of open and closed-ended questions.

The questionnaire was then presented to two other supervisors that were experienced in paediatric research in addition to clinical research to approve the questions and provide any suggestions that they may have to improve the level of validity and reliability.

After the children were assigned to the groups the parents/caregivers were given a questionnaire (Appendix 9) to complete. The questionnaire investigated how long the child had been receiving treatment in the department in order to estimate the number of treatment courses received. They were also asked about the type of services that they have been receiving and asked to rate the different services they had received, such as the intensive therapy, conventional therapy, hydrotherapy and casting.

\subsection{Home Program Questionnaire}

Children receiving physiotherapy in the pa

ediatric department receive a home treatment program as part of the complete management policy of the child. A questionnaire (Appendix 10) regarding the parents/caregivers' views of the home program that they received in the previous period of 
treatment in the centre was created. The questionnaire was developed to assess the home treatment program. The questionnaire was initially developed according to the researcher's base of professional experience in clinical services. The initial format of the questionnaire was refined through discussion with colleagues to improve and develop the depth of the questions and enhance the layout and formatting of open and closed-ended questions.

The questionnaire was then presented to two other supervisors that were experienced in paediatric research in addition to clinical research to approve the questions and provide any suggestions that they may have to improve the level of validity and reliability.

The questionnaire was intended to identify the attitudes of the parents/caregivers toward home treatment, adherence, appreciation, and belief in its effect. This was for the purpose of comparing the views and opinions with the results of the trial and the home program applied as part of the trial. The questionnaire assessed whether the parents received a home program during the period that they have been receiving therapy in the department and in which format have they been receiving it. The questionnaire also assessed whether the parents/caregivers practised the program with their therapist and if further explanation and training had been offered to them by their therapist. Further information was sought to determine whether they understood the purpose and the importance of learning and applying for the home program. Other questions assessed the level of commitment of the parents to the home program and if they had any difficulty in applying it. Finally, the parents were asked whether they observed any benefit from applying for the home program.

\subsubsection{Ethics}

The study was approved by the ethics committee of the School of Medicine in Swansea University (REC) in 2015 (Appendix 5). Since the study was run in Kuwait it did not require ethical approval from the NHS Research Ethics Committee or approval from the $\mathrm{R}+\mathrm{D}$ office. An Ethical Approval was sought from the Ministry of Health in Kuwait with a translated copy found in Appendix 4.

Confidentiality was ensured for all participants in this pilot study. The data collected from participants was recorded anonymously through the use of codes, meaning that no names or personal information were required during data collection, analysis or reporting. The information gathered was used anonymously, with the original documents kept in a 
locked record drawer that only the researcher had access to. All the data gathered will be destroyed 2 years after completing this research.

\subsubsection{Analysis}

This section will be presenting the analysis of the following outcome:

\subsubsection{Previous Service Experience Questionnaire and Home Program Questionnaire}

Quantitative data retrieved from the closed questions within the questionnaire were explored using descriptive statistics and was displayed graphically.

Qualitative data, such as responses to free text questions in the questionnaire were recorded and evaluated using iterative analysis. Comparisons of responses were made to generate themes for discussion later on in the research.

\subsubsection{Analysis of Trial Outcomes}

Analysis of trial data was performed using SPSS (IBM SPSS Statistics, version 22); descriptive statistics were used to analyse the results of the service evaluation questionnaire and the home program questionnaire. Descriptive statistics were also used to analyse the results of the measurements of the GMFM for each child across the three assessments. The outcomes of the GMFM were compared for each child in the three assessments performed and compared with other children in the same group. Differences found in each group were then compared with other groups' measures. Findings were displayed graphically.

For a sample size smaller than 50 such in this study the suitable test of normality would be the Shapiro-Wilk test (Moye, 2013). We observed a p-value of 0.646 for GMFM and so opted to use parametric statistics (Moye, 2013).

Parametric tests were preferred as they are considered to be more powerful than nonparametric tests (Armitage et al, 2008). P-values that are $(>0.05)$ indicate the use of parametric statistics compared to p-values that are not Normal $(<0.05)$ which indicate the need for nonparametric statistics to analyse the data. Homogeneity of the variance and independent samples are also factors that indicate the use of parametric statistics which is not 
the case when using nonparametric statistics (Norman and Streiner, 2003). All p-values calculated below using the Shapiro-Wilk test normalizations tests were found to be normal which indicated that the baseline data for GMFM for each experimental group was not significantly different from the expected theoretical normal distribution; therefore, the GMFM data for all three experimental groups can be analysed using parametric data analysis tests. According to that and to the information found in the literature (Norman \& Streiner, 2003, Moye, 2013) parametric statistics was approved to be the type of statistics used to analyse the data in this study.

\subsection{Participant Characteristics}

Thirty-three children (13 girls and 20 boys) were allocated randomly to the three groups. Initial group allocation had included 11 children in group A, 12 children in group B and 10 children in group $\mathrm{C}$ (Figure 6.18). The levels of CP had varied from I-III on the GMFCS which a common tool is used to classify the level of severity of $\mathrm{CP}$ in children (Refer to chapter 1).

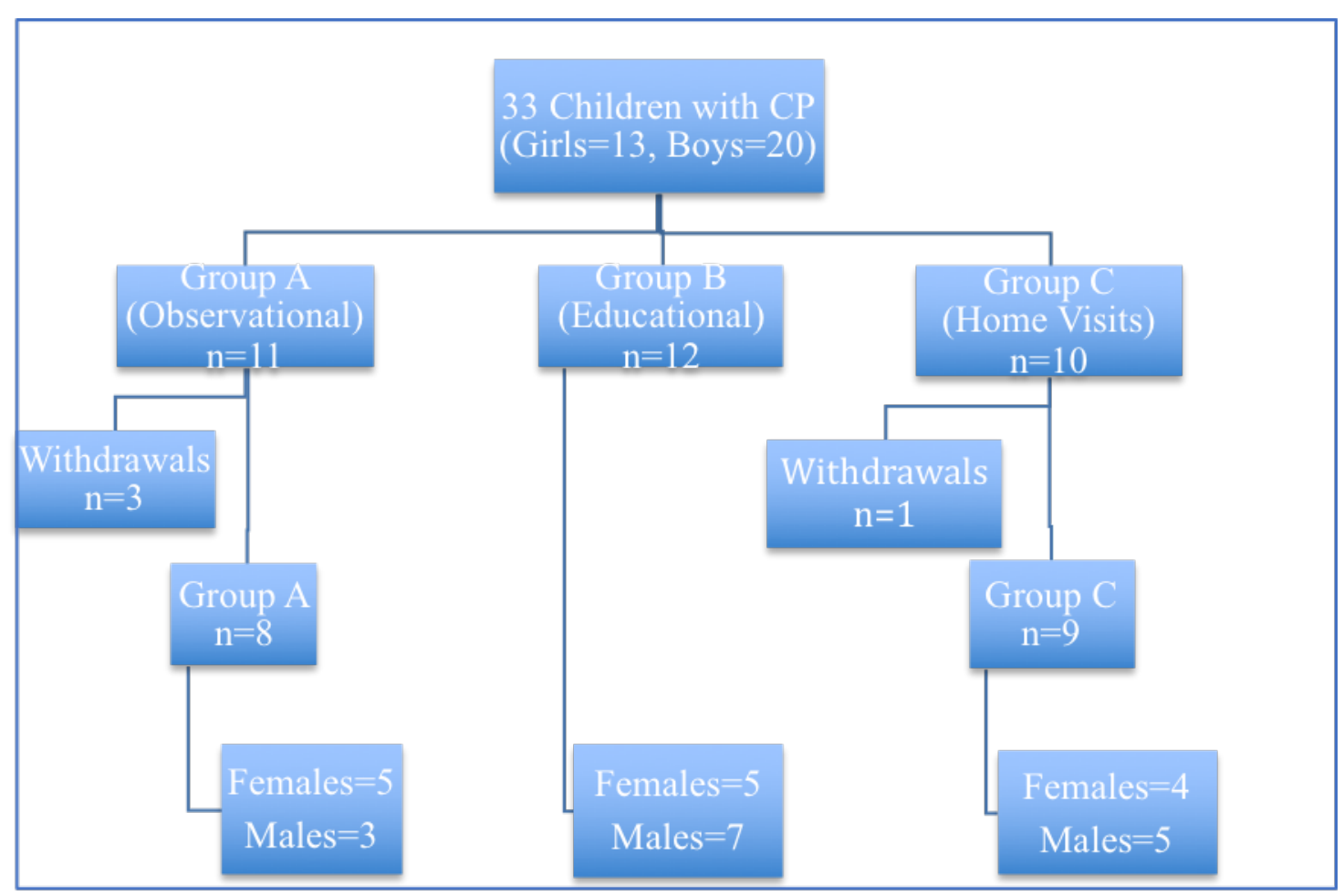

Figure. 6.4 Showing the random allocation of patients to the groups and the number of dropouts from the trial. 
During the trial, 4 children dropped out of the study. Two children from group A (Observational group) missed three physiotherapy sessions in a row in the first six weeks of clinical physiotherapist led care and were consequently withdrawn from the study. Another child from group A had to leave the country for personal issues in the six -week period of no physiotherapist led care. Finally, a child from group C (Home Visit Group) was admitted to the hospital in the six weeks of the physiotherapist led care period and had to be withdrawn from the study. The data for these children were not included in the analysis and all records relating to them was removed to ensure confidentiality. The 29 children remaining in the study have included three boys and five girls in group A of GMFCS levels I (one child), level II (five children), and level III (two children). Children in group B had included seven boys and five girls, of level I (four children), level II (two children) and level III (six children). In group C, the children included five boys and four girls of GMFCS levels I (four children), level II (three children) and level III (two children). The average age for each group was 2.5, 2.4, and 2.1 for groups $\mathrm{A}, \mathrm{B}$, and $\mathrm{C}$ respectively. 


\subsection{Results}

\subsubsection{Service Evaluation Questionnaire}

The duration in which the children have been receiving physiotherapy treatment was recorded since the child was first referred to the physiotherapy department (Q1). This was to observe the duration that the parents have been receiving the services in order for them to establish an opinion of the quality of services provided. Results were $46 \%$ (16/33 participant) 6-12 months, 32\% (10/33) longer than 12 months, 15\% (5/33) and 7\% (2/33) (up to six months) and (one to three months) respectively. This shows a majority (31/33) of the participants that already received at least 2 physiotherapy courses. Therefore, the participants were expected to have a view regarding the services they were receiving.

If the children have previously received an intensive physiotherapy block (six weeks of daily sessions) prior to the study the parents were asked to evaluate the quality of the intervention block on a five-point scale ranging from very good to very bad.

Only 8 participants were found to have received intensive therapy prior to the trial.

Four children that received intensive physiotherapy had indicated the intervention to be 'Very Good'. The parents of 2 children have indicated the intervention being 'Good'. Only one had indicated the intervention as being 'Fair' and 1 had indicated the intervention as being 'Very Bad' (Q2). Although the parents did not mention the reason for the negative feedback the daily intensive physiotherapy sessions may reflect a sense of exhaustion upon the parents as well as the children given that the child may receive a 75 -minute session daily for up to six weeks.

The participants receiving Conventional (as opposed to intensive) physiotherapy was also asked to rate the intervention. The majority of participants $(57 \%)(18 / 33)$ have rated the intervention as being "Very Good" compared to the participants that rated the intervention as being "Good" and "Fair" with percentages of 29\% (10/33) and 14\% (5/33) respectively. None of the participants has rated the conventional treatment course as being "Bad" or "Very Bad" (Q3). This shows an opposing opinion from results of question 2 showing that perhaps the conventional therapy showed better acceptance than the intensive therapy provided.

None of the participants has completed questions four, five and six which were evaluations for Hydrotherapy, Gait Training and Casting respectively. 


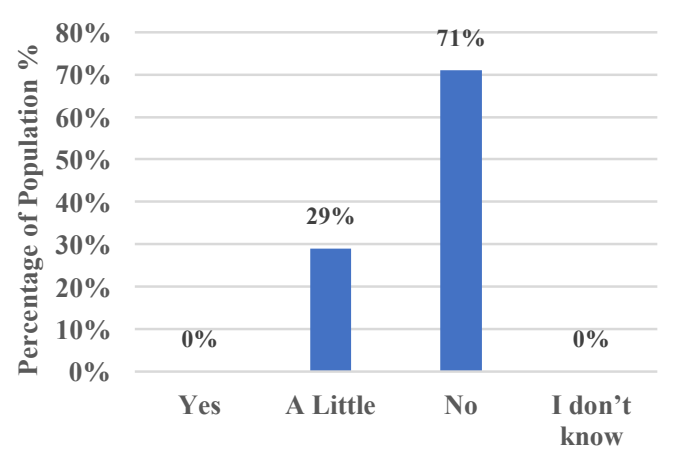

Figure. 6.5 Was there any difficulty in coping with the frequency of bringing your child to the sessions? (Q7)

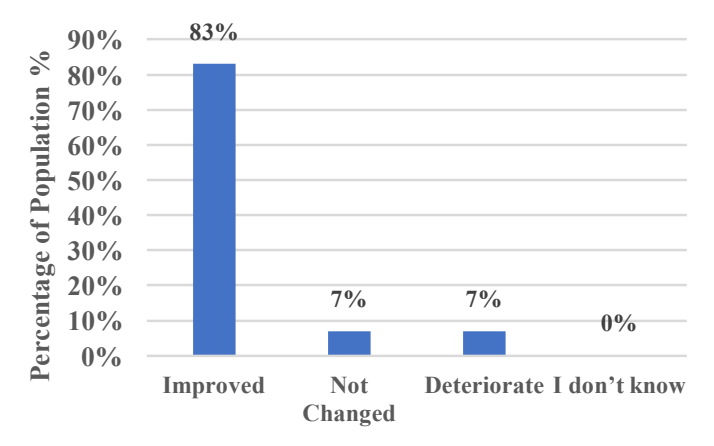

Figure. 6.6 Were there any differences observed in your child's condition? (Q8)

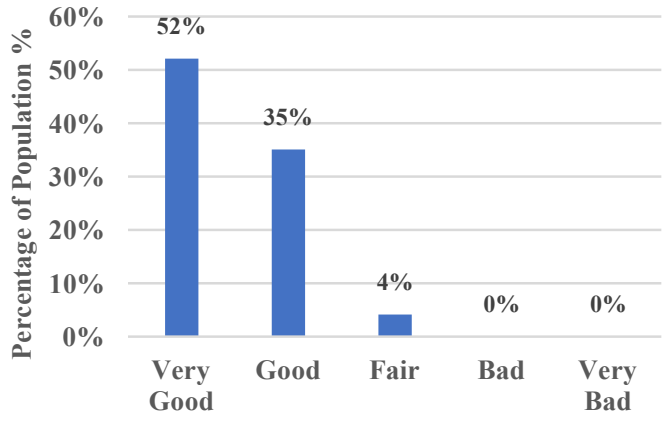

Figure 6.7 If you have received an education course, can you rate it? (Q10) 
More than $71 \%(23 / 33)$ of the total population indicated in fig 6.5 that there was no difficulty experienced in coping with the frequency of the treatment sessions while $29 \%$ $(10 / 33)$ had indicated facing some difficulty in attending the treatment sessions (Q7). Which may reflect the population that had received intensive therapy and the had previously expressed their negative views regarding the quality of intensive physiotherapy.

Fig 6.6 shows the results of Q8 that states improvement in 83\% (27/33), while the rest were divided to not seeing any change or in some cases deterioration.

All participants reported receiving some kind of education informing them on the nature of $\mathrm{CP}$, the prognosis for their children and the importance of home treatment throughout all the physiotherapy courses that they have been receiving (Q9) which reflects that there is some method of education being delivered in the department, however, the method varied from one participant to the other and therefore it was difficult to evaluate the validity of each method. The parents were therefore expected to show some knowledge regarding the importance of the home treatment and some commitment to applying it.

When rating the education that they have received in previous physiotherapy courses $52 \%(17 / 33)$ of the participants that received the education shown in fig 6.7 had reported the education to be 'Very Good'. Also, 35\% (11/33) had reported the education to be 'Good' and $4 \%(1 / 33)$ had decided that the education was 'Fair' (Q10).

None of the participants filled in the open-ended questions of additional suggestions to improve the level of education and physiotherapy services that have been provided at the end of the questionnaire (Q11,12).

In summary, most of the participants have been receiving physiotherapy services for more than 6 months, and only 8 participants have received a course of intensive physiotherapy that was rated as 'Very Good' by half of them. Also, half of the participants rated conventional therapy as 'Very Good'. The majority of the participants had no difficulty with the frequency of treatment sessions. Results indicate that parents were happy with the quality of services provided as well as reporting receiving a method of education of the importance of physiotherapy and the home treatment. 


\subsubsection{Home Program Questionnaire}

Ninety percent of the total participants received a home program in previous treatment courses (Q1) (Fig 6.8). All participants in the questionnaire have agreed to accept a home treatment program if they were offered one as a response to Q2. These results have reflected the positive attitude of the participants and how keen they were to be committed to home treatment.

Figure (6.9) shows that only $77 \%$ (25/33) of the total population that received a home program had received a documented copy while $23 \%$ (7/33) had indicated otherwise (Q3).

In figure (6.10) 89\% (29/33) had indicated that the home program was explained clearly to them compared to the $11 \%(3 / 33)$ that indicated that the home program was not clearly explained (Q4).

The exercises were reported to have been demonstrated to $93 \%(30 / 33)$ of the total population compared to only $7 \%(2 / 33)$ in fig. (6.11) who did not have the exercises demonstrated to them (Q5).

Question 6 in the questionnaire had evaluated the number of participants that have had the chance to practice the home program with the therapist which was found to be a total of $89 \%(29 / 33)$ compared to the $11 \%(3 / 33)$ that did not have that chance (Figure 6.12).

The parents thought that the therapists were open and available for any inquiries and questions that they had in more than $93 \%$ (30/33) of the cases in the total population included compared to the $7 \%(2 / 33)$ that have reported that the physiotherapist did not show great responses for any additional enquiries (Fig 6.13) (Q7).

In fig (6.14) the results of Q8 had indicated that 96\% of the total population have had their therapists indicate the importance of applying the home program compared to the $4 \%$ that have not. All participants have agreed that the exercises were easy to apply in question 9. 


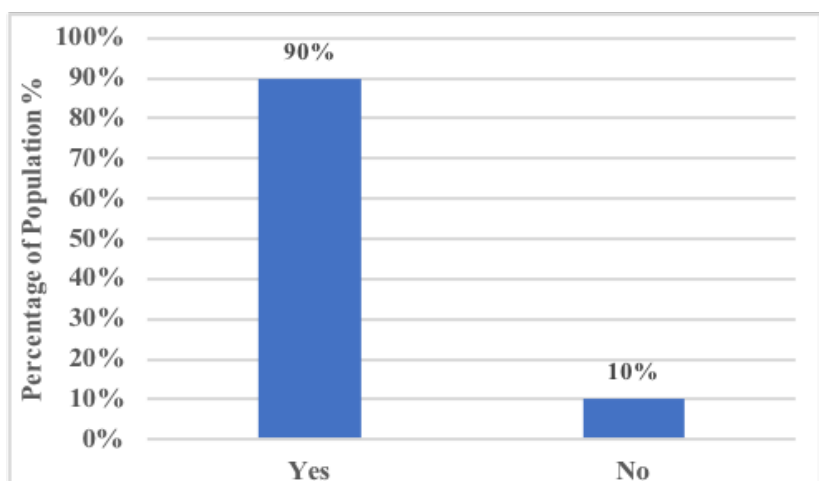

Figure. 6.8. Were you given a home program to apply at home after the sessions took place? (Q1)

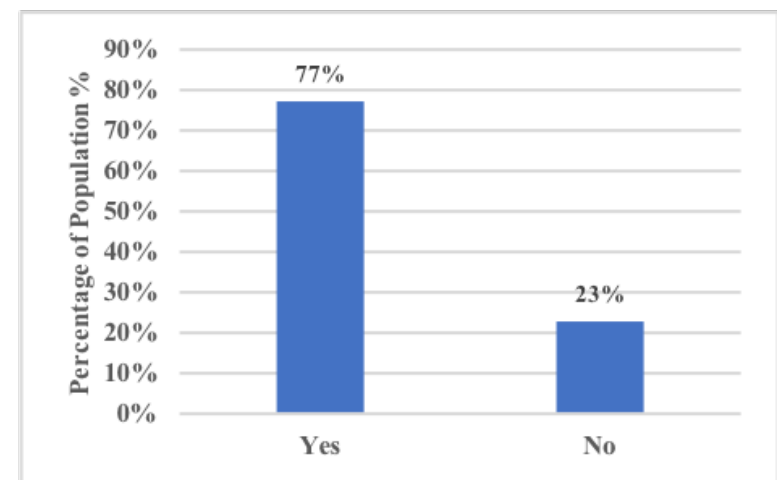

Figure 6.9 Was the home program given to you documented on paper? (Q3) 


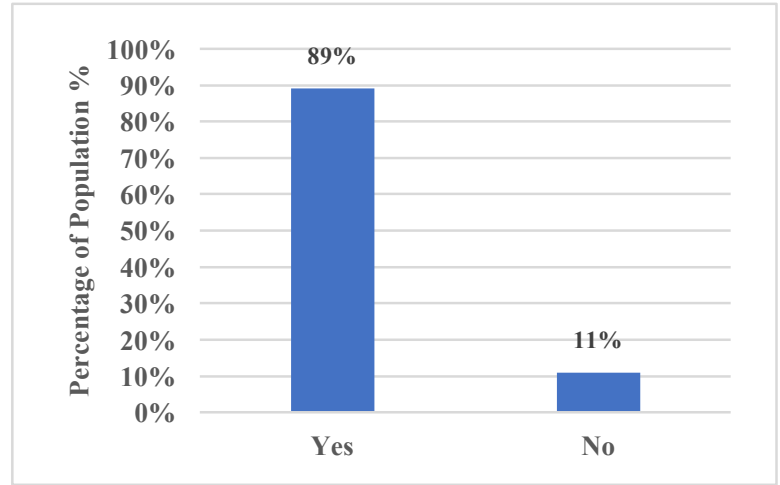

Figure 6.10 Were the exercises of the home program clearly explained? (Q4)

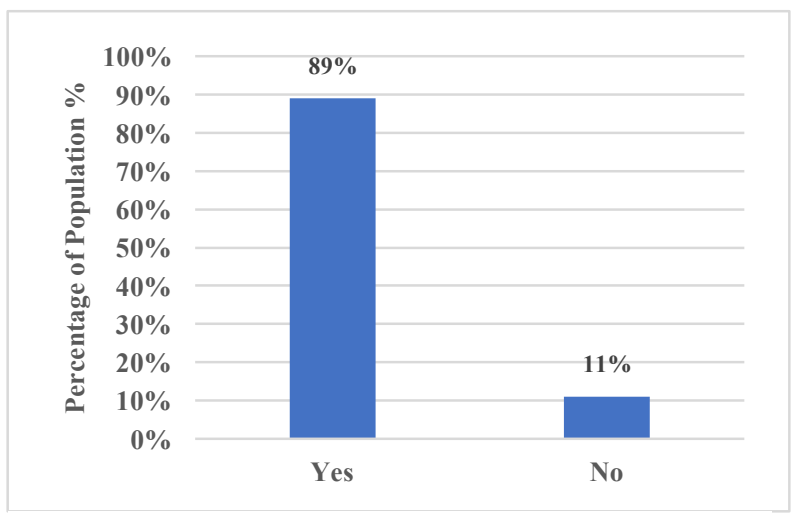

Figure 6.12 Were you given the proper time to understand and practice the exercises with the physiotherapist after receiving the exercises? (Q6)

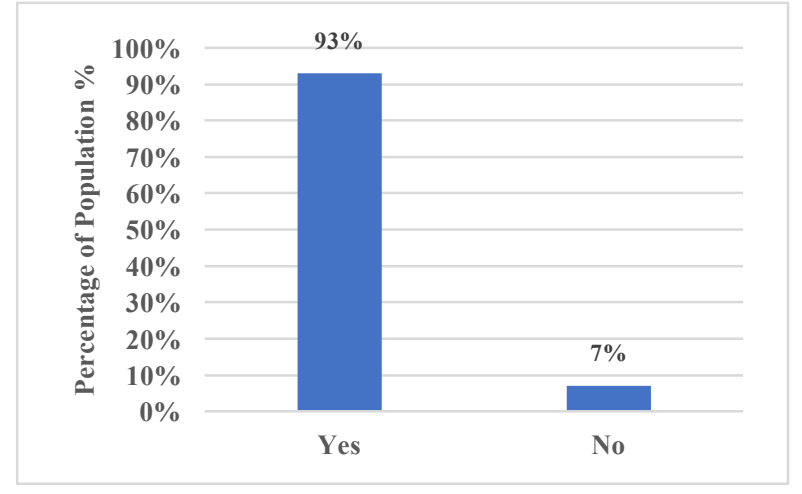

Figure 6.11 Were the exercises demonstrated to you? (Q5)

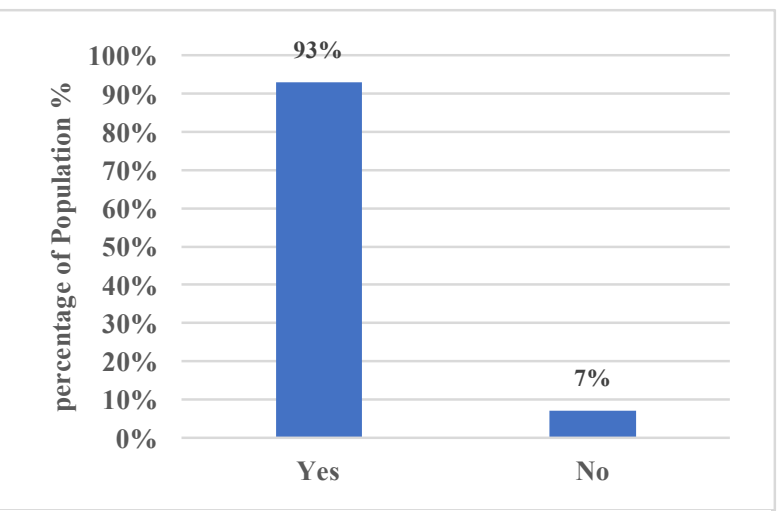

Figure 6.13 Was the physiotherapists open to any inquiries and question regarding the home program? (Q7) 


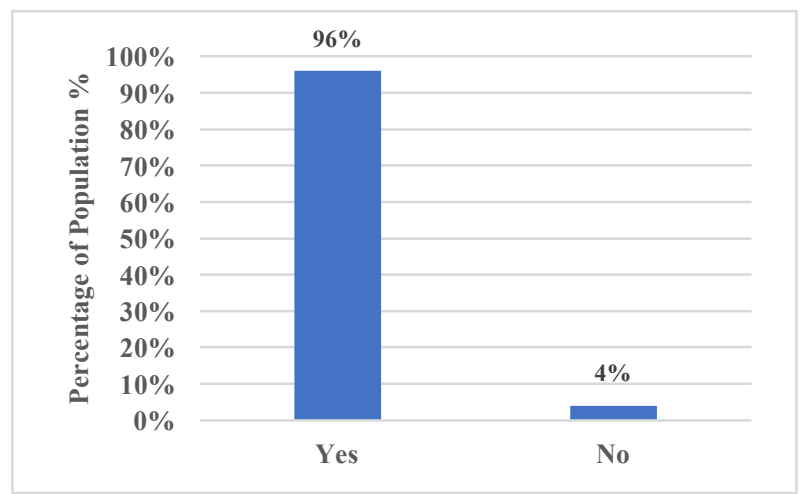

Figure 6.14 Did the physiotherapist explain the purpose and the importance of understanding and applying the home program in the correct frequency and intensity? (Q8)

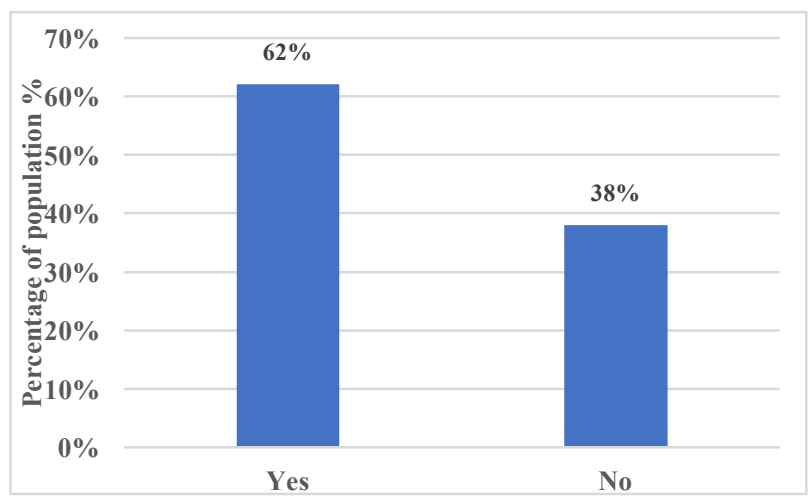

Figure 6.15 Were you committed to the frequency of application? (Q10)

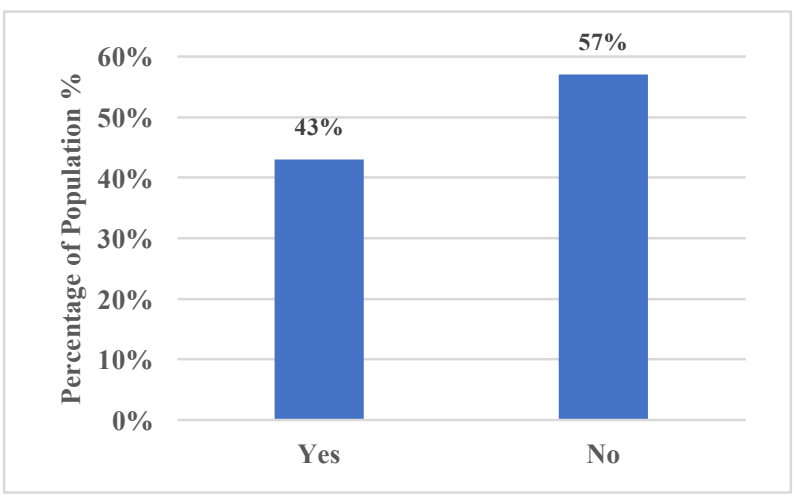

Figure 6.16 Did you find any difficulties in applying the home treatment? (Q11)

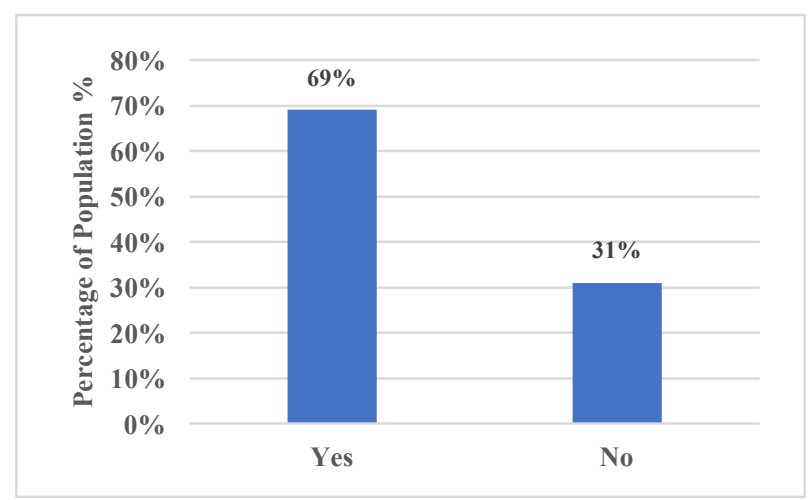

Figure 6.18 Did you see any changes in your child's condition? (Q13)

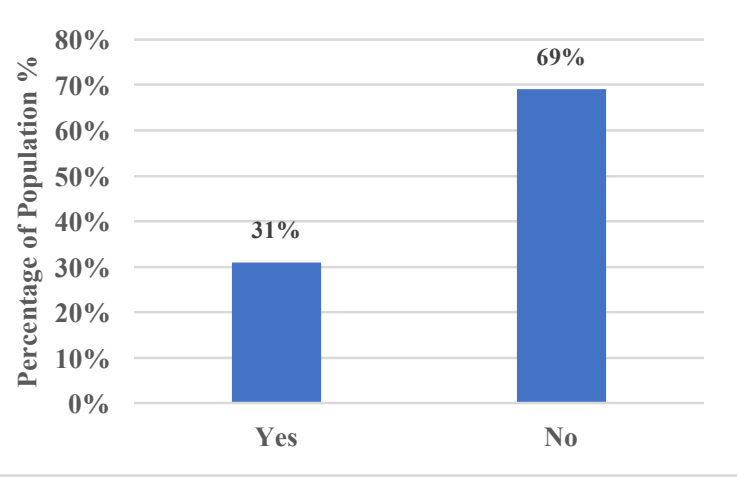

Figure 6.17 Was the home program stressful? (Q12) 
The results of questions 3-9 showed a good effort in presenting the home treatment as well as explaining the importance of applying the home treatment. In response, the parents were expected to show a good level of adherence to the home treatment which was reported in Q10.

However, the parents reported being committed to applying the home treatment in only $62 \%$ of the total population that has received the home program $(\mathrm{Q} 10)$. More than $38 \%$ had reported not being committed to the application of the home program at home even though all the participants have reported previously that the exercises were found simple and easy to apply (fig 6.15).

Figure (6.16), 57\% (19/33) of the total participants have reported that the program was not difficult to apply. However, $43 \%$ (14/33) had reported some difficulty in applying for the program as a whole $(\mathrm{Q} 11)$.

As for the program is a burden to complete, fig (6.17) had shown that $31 \%(10 / 33)$ had found the program to be stressful while 69\% (23/33) had not (Q12).

Questions 10-12 surprisingly did not reflect the level of satisfaction and adherence to the home treatment by the parents that was expected from the level of education that was given by the physiotherapists. This was found to affect the changes observed in the child's condition reported in Q13.

Data results indicate that $69 \%(23 / 33)$ of the population noticed changes in the conditions of their children in response to the application of the home program compared to the $31 \%(10 / 33)$ that haven't noticed any changes (fig 6.18, Q13).

Finally, question 14 reports that all changes that were noticed in their children's conditions were positive.

In summary, results show that the participants have received a home program in previous physiotherapy courses in different methods with a documented paper being the most common. The home program was reported by parents to be clear and not complicated due to being explained and demonstrated in most of the cases by the physiotherapist. The majority of the participants have reported that they have applied the home treatment due to learning its importance and effects from the physiotherapist and the program being easy to apply. Positive changes were detected as a result of applying for the home program by the parents. 
These results referred to a missing link that caused the lack of adherence to the home treatment as well as the limited changes observed in the children's conditions.

\subsubsection{Assessing the Impact of the Parameters of the Trial on the GMFM Scores}

The main outcome measure of the study was the GMFM where measurements were taken before a treatment course of six weeks (Baseline), after a treatment course of six weeks (Post Treatment) and after a six week off period (End of Trial) (Table 6.2).

Table 6.2 Showing the GMFM measures for the children in Group A (Observational Group) with the first three columns representing raw measures of GMFM.

\begin{tabular}{|l|l|l|l|l|l|l|}
\hline Pt & Baseline & $\begin{array}{l}\text { Post } \\
\text { Treatment } \\
\text { Measure }\end{array}$ & $\begin{array}{l}\text { End of } \\
\text { Trial } \\
\text { Measure }\end{array}$ & $\begin{array}{l}\text { Diff b/t Base } \\
\text { and End of } \\
\text { Treat. } \\
\text { Measures }\end{array}$ & $\begin{array}{l}\text { Diff b/t } \\
\text { Baseline and } \\
\text { End of Trial } \\
\text { Measures }\end{array}$ & $\begin{array}{l}\text { Diff b/t } \\
\text { End of } \\
\text { Treatment } \\
\text { and End of } \\
\text { Trial }\end{array}$ \\
\hline 1 & 59.00 & 62.65 & 57.50 & 3.65 & -1.50 & -5.15 \\
\hline 2 & 67.42 & 72.28 & 72.94 & 4.86 & 5.52 & .66 \\
\hline 3 & 39.30 & 63.00 & 73.00 & 23.70 & 33.70 & 10.00 \\
\hline 4 & 65.12 & 76.52 & 73.24 & 11.40 & 8.12 & -3.28 \\
\hline 5 & 56.10 & 63.90 & 51.45 & 7.80 & -4.65 & -12.45 \\
\hline 6 & 91.94 & 94.26 & 94.82 & 2.32 & 2.88 & .56 \\
\hline 7 & 98.64 & 99.44 & 98.60 & .80 & -.04 & -.84 \\
\hline 8 & 53.95 & 59.50 & 56.00 & 5.55 & 2.05 & -3.50 \\
\hline
\end{tabular}


Table 6.3 Showing the GMFM measures for the children in Group B (Educational Group) with the first 3 columns representing raw measures of GMFM.

\begin{tabular}{|c|c|c|c|c|c|c|}
\hline $\mathrm{Pt}$ & $\begin{array}{l}\text { Baseline } \\
\text { Measure }\end{array}$ & $\begin{array}{l}\text { Post } \\
\text { Treatment } \\
\text { Measure }\end{array}$ & $\begin{array}{l}\text { End of } \\
\text { Trial } \\
\text { Measure }\end{array}$ & $\begin{array}{l}\text { Diff } b / t \text { Base and } \\
\text { End of Treat. } \\
\text { Measures }\end{array}$ & $\begin{array}{l}\text { Diff } b / t \text { Baseline } \\
\text { and End of Trial } \\
\text { Measures }\end{array}$ & $\begin{array}{l}\text { Diff } b / t \text { End } \\
\text { of Treatment } \\
\text { and End of } \\
\text { Trial }\end{array}$ \\
\hline 1 & 83.70 & 85.80 & 89.40 & 2.10 & 5.70 & 3.60 \\
\hline 2 & 53.10 & 63.20 & 76.50 & 10.10 & 23.40 & 13.30 \\
\hline 3 & 85.40 & 84.80 & 88.80 & -.60 & 3.40 & 4.00 \\
\hline 4 & 51.90 & 66.18 & 69.28 & 14.28 & 17.38 & 3.10 \\
\hline 5 & 63.40 & 68.40 & 72.30 & 5.00 & 8.90 & 3.90 \\
\hline 6 & 26.36 & 42.38 & 45.10 & 16.02 & 18.74 & 2.72 \\
\hline 7 & 56.80 & 58.90 & 62.90 & 2.10 & 6.10 & 4.00 \\
\hline 8 & 30.15 & 45.10 & 51.72 & 14.95 & 21.57 & 6.62 \\
\hline 9 & 73.50 & 80.42 & 85.70 & 6.92 & 12.20 & 5.28 \\
\hline 10 & 53.90 & 56.00 & 61.70 & 2.10 & 7.80 & 5.70 \\
\hline 11 & 80.76 & 84.60 & 85.10 & 3.84 & 4.34 & .50 \\
\hline 12 & 89.00 & 90.30 & 92.20 & 1.30 & 3.20 & 1.90 \\
\hline
\end{tabular}

Table 6.4 Showing the GMFM measures for the children in Group C (Home Visit Group) with the first 3 columns representing raw measures of GMFM.

\begin{tabular}{|l|l|l|l|l|l|l|}
\hline Pt & $\begin{array}{l}\text { Baseline } \\
\text { Measure. }\end{array}$ & $\begin{array}{l}\text { Post } \\
\text { Treatment } \\
\text { Measure. }\end{array}$ & $\begin{array}{l}\text { End of Trial } \\
\text { Measure. }\end{array}$ & $\begin{array}{l}\text { Diff b/t Base } \\
\text { and End of } \\
\text { Treat. } \\
\text { Measures }\end{array}$ & $\begin{array}{l}\text { Diff b/t } \\
\text { Baseline and } \\
\text { End of Trial } \\
\text { Measures }\end{array}$ & $\begin{array}{l}\text { Diff b/t End of } \\
\text { Treatment and } \\
\text { End of Trial }\end{array}$ \\
\hline 1 & 58.40 & 63.22 & 64.50 & 4.82 & 6.10 & 1.28 \\
\hline 2 & 32.30 & 35.00 & 38.98 & 2.70 & 6.68 & 3.98 \\
\hline 3 & 63.70 & 66.80 & 68.80 & 3.10 & 5.10 & 2.00 \\
\hline 4 & 39.30 & 62.10 & 61.16 & 22.80 & 21.86 & -.94 \\
\hline
\end{tabular}




\begin{tabular}{|l|l|l|l|l|l|l|}
\hline 5 & 72.90 & 78.62 & 79.96 & 5.72 & 7.06 & 1.34 \\
\hline 6 & 33.36 & 39.10 & 45.24 & 5.74 & 11.88 & 6.14 \\
\hline 7 & 69.34 & 73.40 & 75.70 & 4.06 & 6.36 & 2.30 \\
\hline 8 & 62.76 & 68.40 & 70.10 & 5.64 & 7.34 & 1.70 \\
\hline 9 & 80.45 & 84.00 & 85.80 & 3.55 & 5.35 & 1.80 \\
\hline
\end{tabular}

The data for each patient participating was standardised (to zero means) to eliminate the variation in the mean level between the participants without affecting the comparisons of change from baseline.

A pre-test/post-test approach was used in the trial to identify the effect of the intervention given in the treatment period and the effect of the intervention applied in the "Off Period" in the same group. A comparison of results between the different groups (Table $6.2,6.3,6.4)$ was then made. In the same group the measures at "Baseline" and "Post Treatment: were compared.

Another comparison was made between groups to identify if there was any significant change between the GMFM values of "Post Treatment" measurements and "End of Trial" measurements.

Within-group comparisons were also made to identify the difference between the values of GMFM at baseline and at the end of the trial after the off period had ended.

Between groups, analyses were then performed to identify if there was any significant difference between the mean values of GMFM between the 3 groups in different measurements.

\subsubsection{Normality Testing}

To test for normality, a Shapiro-Wilk analysis was conducted on the raw baseline data to identify the choice of parametric or nonparametric tests to apply according to the p-value (Table 6.5). 
Table 6.5 Test of normality for baseline measures for all data

\begin{tabular}{|l|r|r|r|r|r|r|}
\hline & \multicolumn{3}{|c|}{ Kolmogorov-Smirnov } & \multicolumn{3}{c|}{ Shapiro-Wilk } \\
\cline { 2 - 7 } & Statistic & \multicolumn{1}{c|}{ df } & \multicolumn{1}{c|}{ Sig. } & Statistic & \multicolumn{1}{c|}{ df } & \multicolumn{1}{c|}{ Sig. } \\
\hline $\begin{array}{l}\text { Baseline } \\
\text { Measurement }\end{array}$ & .097 & 29 & $.200^{*}$ & .973 & 29 & .646 \\
\hline
\end{tabular}

The p-value used as follows in tables (6.6), (6.7), (6.8) for each group of treatment:

Table 6.6 Test of normality for baseline measures of group A (Observational).

\begin{tabular}{|l|r|c|c|r|r|r|}
\hline \multirow{2}{*}{} & \multicolumn{3}{|c|}{ Kolmogorov-Smirnov } & \multicolumn{3}{|c|}{ Shapiro-Wilk } \\
\cline { 2 - 7 } & Statistic & df & Sig. & Statistic & df & Sig. \\
\hline $\begin{array}{l}\text { Baseline } \\
\text { Measurement }\end{array}$ & .230 & 8 & $.200^{*}$ & .920 & 8 & .429 \\
\hline
\end{tabular}

Table 6.7 Tests of normality for the baseline measures of Group B (Educational Group).

\begin{tabular}{|l|r|r|r|r|r|r|}
\hline & \multicolumn{3}{|c|}{ Kolmogorov-Smirnov } & \multicolumn{3}{|c|}{ Shapiro-Wilk } \\
\cline { 2 - 7 } & Statistic & df & Sig. & Statistic & df & Sig. \\
\hline $\begin{array}{l}\text { Baseline } \\
\text { Measurement }\end{array}$ & .145 & 12 & $.200^{*}$ & .924 & 12 & .320 \\
\hline
\end{tabular}

Table 6.8 Tests of normality for the baseline measures of Group C (Home Visit Group).

\begin{tabular}{|l|r|r|r|r|r|r|}
\hline & \multicolumn{3}{|c|}{ Kolmogorov-Smirnov } & \multicolumn{3}{|c|}{ Shapiro-Wilk } \\
\cline { 2 - 7 } & Statistic & df & Sig. & Statistic & df & Sig. \\
\hline $\begin{array}{l}\text { Baseline } \\
\text { Measurement }\end{array}$ & .199 & 9 & $.200^{*}$ & .901 & 9 & .258 \\
\hline
\end{tabular}




\subsubsection{Statistical Analysis}

One-way analysis of variance (ANOVA) was used to compare means of the three groups at baseline, post-treatment, and post-trial. The one-way ANOVA statistical test is usually selected to determine whether there are any significant differences statistically between three or more independent groups by comparing the means of these groups (Plishta \& Garzon, 2009).

Table 6.9 Comparisons between GMFM results

\begin{tabular}{|c|c|c|c|c|}
\hline & & $\mathrm{df}$ & Mean Square & Sig. \\
\hline $\begin{array}{l}\text { Base measures } \\
\text { of GMFM }\end{array}$ & $\begin{array}{l}\text { Within Groups } \\
\text { Total }\end{array}$ & $\begin{array}{l}26 \\
28\end{array}$ & .000 & \\
\hline $\begin{array}{l}\text { Difference of } \\
\text { GMFM } \\
\text { measures } \\
\text { between the } \\
\text { base and end } \\
\text { of the clinical } \\
\text { physio course }\end{array}$ & $\begin{array}{l}\text { Between Groups } \\
\text { Within Groups } \\
\text { Total }\end{array}$ & $\begin{array}{r}2 \\
26 \\
28\end{array}$ & $\begin{array}{r}3.034 \\
41.026\end{array}$ & .929 \\
\hline $\begin{array}{l}\text { Difference of } \\
\text { GMFM } \\
\text { measures } \\
\text { between base } \\
\text { and end of } \\
\text { Trial }\end{array}$ & $\begin{array}{l}\text { Between Groups } \\
\text { Within Groups } \\
\text { Total }\end{array}$ & $\begin{array}{r}2 \\
26 \\
28\end{array}$ & $\begin{array}{l}67.724 \\
70.329\end{array}$ & .395 \\
\hline $\begin{array}{l}\text { Difference of } \\
\text { GMFM } \\
\text { measures } \\
\text { between end } \\
\text { of clinical } \\
\text { physio course } \\
\text { and end of } \\
\text { the trial }\end{array}$ & $\begin{array}{l}\text { Between Groups } \\
\text { Within Groups } \\
\text { Total }\end{array}$ & $\begin{array}{l}2 \\
26 \\
28\end{array}$ & $\begin{array}{l}95.373 \\
16.369\end{array}$ & .008 \\
\hline
\end{tabular}


The one-way ANOVA (Table 6.9) only shows a significant difference between the values of GMFM measurements of post-treatment measurements and post-trial. However, the test does not refer the significance to any groups.

An additional test known as the Post-Hoc test is run to confirm where the differences occurred between groups. The post-Hoc test is known as the posterior tests that are performed after the significance have been shown by a one-way ANOVA test. More than one type of Post-Hoc test is available. The choice would depend on the type of data of the study. The most common tests are considered to be the Tukey Honestly Significant Difference test and the Bonferroni t-test. The Tukey test usually assumes that all the groups included in the analysis are of the same size which is not always the case in trials. The Bonferroni t-test is considered a more suitable choice when comparing a small number of means as it is known to be more sensitive to that situation, whereas the Tukey test is considered in the incidence of making a large number of comparisons. Another reason for this choice is the homogeneity of variance in the measures (Plishta \& Garzon, 2009). The Bonferroni t-test was, therefore, chosen as the post-hoc test for the one-way ANOVA for these measurements.

Table 6.10 Results of Bonferroni test

\begin{tabular}{|c|c|c|c|c|c|c|}
\hline \multirow[t]{2}{*}{ Dependent Variable } & \multirow[b]{2}{*}{ (I) Group } & \multirow[b]{2}{*}{ (J) Group } & \multirow[b]{2}{*}{ Mean Difference (I-J) } & \multirow[b]{2}{*}{ Sig. } & \multicolumn{2}{|c|}{ 95\% Confidence Interval } \\
\hline & & & & & Lower Bound & Upper Bound \\
\hline \multirow{6}{*}{$\begin{array}{l}\text { Difference of GMFM } \\
\text { measures between the } \\
\text { base and end of the } \\
\text { clinical physio course }\end{array}$} & B & $\mathrm{C}$ & .05028 & 1.000 & -7.1773 & 7.2778 \\
\hline & & A & -1.00083 & 1.000 & -8.4820 & 6.4804 \\
\hline & $\mathrm{C}$ & $\mathrm{B}$ & -.05028 & 1.000 & -7.2778 & 7.1773 \\
\hline & & A & -1.05111 & 1.000 & -9.0155 & 6.9132 \\
\hline & A & $\mathrm{B}$ & 1.00083 & 1.000 & -6.4804 & 8.4820 \\
\hline & & $\mathrm{C}$ & 1.05111 & 1.000 & -6.9132 & 9.0155 \\
\hline \multirow{3}{*}{$\begin{array}{l}\text { Difference of GMFM } \\
\text { measures between } \\
\text { base and end of Trial }\end{array}$} & B & $\mathrm{C}$ & 2.42417 & 1.000 & -7.0387 & 11.8871 \\
\hline & & A & 5.30083 & .534 & -4.4942 & 15.0959 \\
\hline & $\mathrm{C}$ & $\mathrm{B}$ & -2.42417 & 1.000 & -11.8871 & 7.0387 \\
\hline
\end{tabular}




\begin{tabular}{|c|c|c|c|c|c|c|}
\hline & & A & 2.87667 & 1.000 & -7.5509 & 13.3043 \\
\hline & A & B & -5.30083 & .534 & -15.0959 & 4.4942 \\
\hline & & $\mathrm{C}$ & -2.87667 & 1.000 & -13.3043 & 7.5509 \\
\hline \multirow{6}{*}{$\begin{array}{l}\text { Difference of GMFM } \\
\text { measures between end } \\
\text { of clinical physio } \\
\text { course and end of trial }\end{array}$} & B & $\mathrm{C}$ & 2.37389 & .585 & -2.1914 & 6.9392 \\
\hline & & A & $6.30167^{*}$ & .006 & 1.5762 & 11.0272 \\
\hline & $\mathrm{C}$ & B & -2.37389 & .585 & -6.9392 & 2.1914 \\
\hline & & A & 3.92778 & .169 & -1.1029 & 8.9585 \\
\hline & A & B & $-6.30167^{*}$ & .006 & -11.0272 & -1.5762 \\
\hline & & $\mathrm{C}$ & -3.92778 & .169 & -8.9585 & 1.1029 \\
\hline
\end{tabular}

The Bonferroni test showed the difference between group A and B to be significant $(p=0.006)$ in the results of the GMFM measures between "End of Clinical Physio Course" and "End of Trial" (Table 6.10).

Results are seen in this following graph:

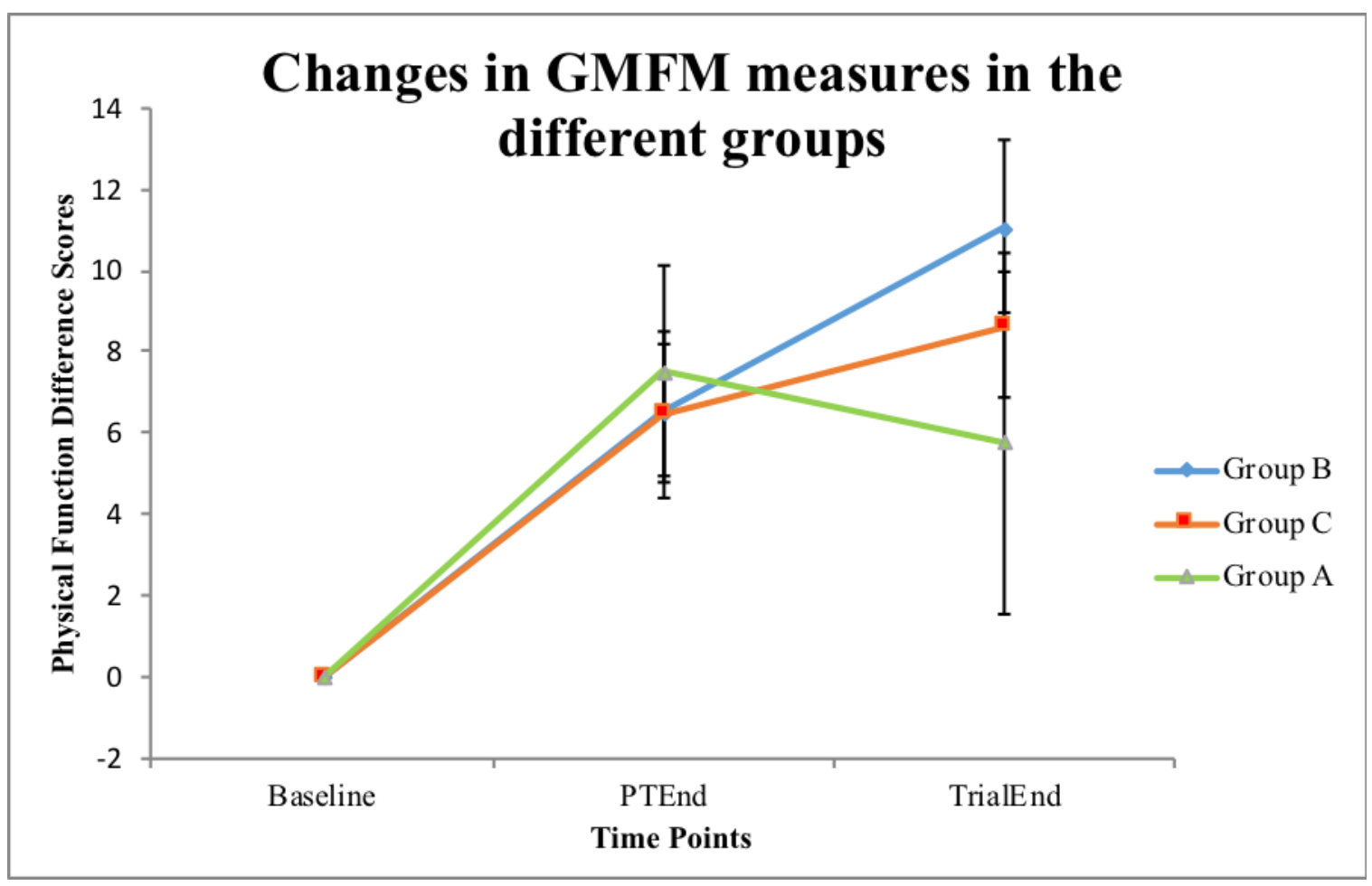

Figure 6.19 Indicating the comparison in improvement in GMFM measures between baseline, second and third assessment for the three groups of children. 
Other than the difference between the post-treatment and post-trial measures of group $\mathrm{A}$ and $\mathrm{B}$, none of the other measures shows any significance.

\subsubsection{Were the Groups the Same Going into the Study?}

In order to evaluate the homogeneity of participants at the beginning of the trial, a comparison was performed between the baseline measures of the participants in the three groups.

Table 6.11 Showing the p-value of the Three Groups at Baseline Measures

\begin{tabular}{ccccccc}
\hline Group & $\boldsymbol{N}$ & Mean & SD & Df & $\boldsymbol{F}$ & $\boldsymbol{P}$ \\
\hline Group 1 & 12 & 62.3308 & 20.86926 & 2 & .501 & .612 \\
Group 2 & 9 & 56.9456 & 17.73844 & 26 & & \\
Group 3 & 8 & 66.4338 & 19.80130 & & & \\
Total & 29 & 61.7914 & 19.30941 & & & \\
\hline
\end{tabular}

No significant difference was found between the participants in the three groups in the baseline measures $(p>0.05)$ and therefore this confirms that the variation between groups at the beginning of the trial is small and unlikely to bias any results (Table 6.11). 


\subsection{Discussion}

The trial was designed to assess several aspects. The questionnaires were used to study the opinions of parents of the services that they have been receiving while the GMFM measures were used to measure the effects of the different support methods provided for the different groups. The literature was explored to identify the parameters of best and common practice of physiotherapy treatment in general and Bobath treatment (see chapter 2). This pilot study was created according to the results of the audit in chapter five to assess the effectiveness of different parameters of applying a home program to support the physiotherapy management provided in the clinic in an effort to improve and maintain the functional level of the children with $\mathrm{CP}$ that are receiving treatment in the clinic in PMR hospital in Kuwait.

\subsubsection{Views and Opinions of Parents}

The views and opinions of parents were investigated in this pilot study to further interpret the results of the audit performed in chapter five. A questionnaire was therefore distributed at the beginning of the pilot study to explore the views and opinions of the parents with respect to previous services that they have received in the paediatric physiotherapy department. Some patients have reported that attending the physiotherapy sessions were in some conditions stressful, this was expected to have a correlation with the number of DNAs found in chapter five. In other words, the stressful process of keeping up with the clinical sessions have shown to lead to absence from physiotherapy sessions and, therefore, cancellation of the courses for children of stressed up parents. This finding had also confirmed the need to focus on implementing the home treatment in order to avoid stress on families which was one of the reasons for implementing the pilot study performed. Another reason justifying the high percentage of DNAs would be the dissatisfaction of parents with the limited progress seen in their child's condition, something reported by some of the participants when speaking about physiotherapy treatment. The limited level of change indicates a limitation that is preventing positive changes in response physiotherapy treatment. It should also be mentioned that the majority of the participants have had at least three courses of physiotherapy treatment in the department and that all the participants of the pilot study were of GMFCS level of III and above and that none of the children included in the pilot was severe in $\mathrm{CP}$ level. This excludes the chance that limitation in progress of 
functional level was due to the severity of the symptoms as implicated by DeGangi and Royeen (1994) or to the fact that the children haven't had enough treatment duration to show progress. However, it may also refer to the intermittent treatment received and the insufficient application of home treatment.

The audit in chapter five showed no documented evidence of education provided to the parents in the file documentation which suggests variation in the level of involvement of parents throughout treatment. Surprisingly this was not the case learned by the questionnaire where the participants reported a fair level of education and implementation of the home treatment by the physiotherapists. The "Service Evaluation" questionnaire and the "Home Treatment" questionnaire (Q3-9) indicated a fair level of education provided to the parents regarding the nature of their children's conditions in addition to the level of progress in the future and care needed at home.

However, the limited levels of commitment in addition to the limited changes seen in the children's conditions (Q10-12) had shown a missing link between education and commitment. The link was expected to be "follow up" and "supervision" that may have shown improved levels of support to the families in the periods in which treatment was delivered at home.

The "Service Evaluation" questionnaire investigating previous experiences of the participants with home programs showed that not all participants received a home program and some parents reporting never receiving a home program. This may have affected the level of application of the home program if the families were receiving it for the first time in the pilot study. In the questionnaire (Q25) some participants reported receiving a limited explanation of the exercises and positions provided by the therapist.

The level of commitment may have also been affected due to another factor missing that was promoted by Knox and Evans (2002) and Butler and Darrah (2001) to improve clinical and home physiotherapy results. The previous studies proposed that family inclusion in goal setting and the management of the child in different environments is a key factor for promoting better results in clinical and home physiotherapy treatment. Basaran et al. (2014) had also promoted follow up and support provided to the parents as one of the most important aspects that would promote adherence to the home program. Therefore, lack of follow up might be expected to affect the awareness of the importance of the application of home treatment resulting in the limited level of adherence. 
This was identified as a limitation that may have also led to the weak prognosis seen in children by their parents.

With regards to the previous home programs participants, only $61 \%$ reported having been committed (Fig 6.15) even though all participants reported that the exercises were simple and easy. Therefore, the issue that had been affecting the adherence to previous home programs had not been the difficulty of the exercises provided. However, the difficulty was reported in applying for the program at home throughout the week leading the parents to be stressed as they have reported in question 11. Also, according to Basaran et al. (2014) stress is considered one of the main factors that prevented parents from adhering to the home program.

\subsubsection{Effectiveness of the Different Parameters of Support to Promote Adherence}

All children included in the pilot study showed improvement in the GMFM scores between baseline and the second assessment, suggesting the effectiveness of the clinical intervention in the first six weeks even though the changes were not significant.

When comparing the second assessment of GMFM measures taken after the six weeks of clinical hands-on physiotherapy and the measures of GMFM taken at the end of the pilot study it is seen in fig (6.19) that there is a clear difference in improvement or maintenance of functional level in the experimental groups compared to the control group. The significant $(p=0.006)$ difference in the final measurement of GMFM between experimental group B and the control group indicates the effectiveness of the support and the education provided for that participant in group B in promoting adherence to the home program and therefore higher levels of the improved functional level. As for group $\mathrm{C}$, even though surprisingly no significant difference was found in the final GMFM measures between experimental group $\mathrm{C}$ and the control group (A) it should be mentioned that group $\mathrm{C}$ had maintained their functional level compared with group A that have shown deterioration in GMFM measures at the end of the pilot study. Therefore, the education and follow up may not have had an effect on increasing functional level but had at least maintained the achieved level from the six -week physiotherapy hands-on period (Difference of GMFM measures between "End of Clinical Physio Course" and "End of Trial of Within Group Measures" (table 6.9)). This qualifies the 
physiotherapist to carry on to improve functional level rather than reset previously achieved goals in order to achieve them again similar to the cases in group A.

Results of the GMFM indicated a significant difference between the baseline and the final GMFM measurement for group B (fig 6.19). Even though the literature (Piggot et al., 2003; Taylor et al., 2004) indicated that follow up would promote a higher level of adherence none of the experiments documented in the literature had provided weekly visits to the families. In this study, the visits might have been overwhelming in group $\mathrm{C}$ (Home Visit Group) and had created a sense of dependence on the researcher's instructions. This had caused the families to apply the home treatment only when the researcher provided the visit. In some cases, when the families are under the stress of caring for a child with special needs in addition to other siblings it results in a high level of pressure. In cases similar to this some parents are unable to be fully committed to applying the home treatment in the frequency advised by the physiotherapist and may develop a sense of dependence on clinical physiotherapy sessions and in this case, they may have depended on the home visit and the training given by the researcher as the only therapy provided at home during the week.

Results of this study therefore indicates the educational lecture as a new intervention in Kuwait to be a more effective intervention in promoting adherence to home treatment and further more promote higher levels of change in outcomes compared to the weekly home visits as the opposing new intervention used in Kuwait for children with CP and their families. It is also considered to be a more practical and cost-effective intervention.

Another aspect from the audit (and the questionnaire of home treatment) that may provide justification for the results of group $\mathrm{C}$ is the lack of experience of the home program from previous physiotherapy courses. The results have reported some parents never received a home treatment program and therefore may have affected their adaptation to apply the home treatment.

Even though the clinical audit revealed that a home program was part of the system applied in the department and that a home program was provided for each child this was not the case in respect to the education, practice, and follow up for parents. The methods of educating families about the home treatment in the department of paediatric physiotherapy needed improvement. This was noticed from the audit and the questionnaire of "Evaluating Home Treatment". According to some studies similar to Knox and Evans (2002) and Tsorlakis et al (2004) a continuous approach of treatment, even though not intensive, had shown the 
highest levels of efficiency. This was a main objective of the pilot study, promoting home treatment to ensure uninterrupted physiotherapy throughout the trial duration.

The choice of GMFM may also have influenced the findings of the study. Even though the GMFM is the most common validated objective measure (Josenby et al., 2009) for change in the functional level of children with $\mathrm{CP}$ it is not considered very sensitive to minor change in functional level (McCarthy et al., 2002; Nelson et al., 2006). Minor changes may be present but cannot be detected by the 88 items of the measure, and may falsely indicate no change. Also in many cases, the GMFM was found to be more sensitive in measuring the change in moderate levels of $\mathrm{CP}$ and was found not to be as sensitive in measuring changes in functional levels of extremely mild or extremely severe cases of CP (Russell et al., 2002; Trahan et al., 1999). Therefore, a more objective and sensitive measurement tool must be implemented in future studies for evaluating functional levels of children with $\mathrm{CP}$. The exclusion of very mild (level I) and very severe (levels IV, V) cases of CP may also help in avoiding the limitations of the measurement tool and its lack of sensitivity in measuring mild changes in such cases even though in another more recent study (Ko, 2014) it was found that the GMFM shows enough sensitivity for CP levels I, II and III and had confirmed the validity to measure changes in the severe levels of IV and V, however, was less sensitive. In this study, however, children with CP of level I was included in an effort to include as many participants as possible. The GMFM was found according to KO in 2014 to be the most valid and reliable outcome measure to evaluate the motor functions in all GMFCS levels of CP.

The duration of the study may have also played a role in affecting the results. The 12 weeks of the trial were suspected to be a limited time for significant changes to be observed especially that some changes were seen in the GMFM measures that were expected to show the significance if the duration of the trial including the "Clinical Physiotherapy Period" and the "Off Period" were extended.

\subsubsection{Feasibility of the Trial}

The pilot experimental trial was deemed to be feasible and was successfully carried out in the paediatric physiotherapy department in PMR hospital in Kuwait for children with CP. Some limitation had been identified and would require correction if a future RCT was to 
be run. Given that the study was a pilot the limited number of participants included may have affected the power of the study.

Difficulty was found in recruiting patients for the study due to the restrictive nature of the inclusion criteria. In addition, children frequently got get sick and in some cases were hospitalized, leading to their absence and therefore withdrawal from the trial. In many cases, the families were not interested in participating in the trial. Many of the children in the department were younger than 1 year of age and therefore did not qualify to be recruited and many have not yet been clearly diagnosed by a paediatrician as a patient with CP. The children in the department had a wide range of variation of the type of $\mathrm{CP}$ and the severity of $\mathrm{CP}$ and therefore difficulty may be found in standardization of the treatment provided. Moreover, the Bobath approach is considered a tailored approach for each child and may not be applied as a standardized method of treatment (Damiano, 2009).

Limitations of the trial study had included:

- The geographic limitation of only including the population receiving treatment in one rehabilitation hospital (although it is the only rehabilitation hospital in Kuwait). Although the other hospitals provide treatment services for the children with $\mathrm{CP}$ the PMR hospital which the trial took place in receives a larger population of children that are referred from a wider regional area in Kuwait.

- Difficulty in finding and recruiting patients meeting the inclusion criterion

- The low proportion of parents prepared to take part in the study.

- Fitting the participants into the schedule of the assessing therapists without interfering with their treatment sessions and without adding an additional burden of attending extra sessions that would stress the parents/caregivers timing.

- The limited number of assessing therapists that have been blind to the procedures of the trial.

- The level of adherence of the parents/caregivers and patients in the clinical treatment sessions and assessment sessions.

- The number of participants withdrawing from the trial due to compulsory circumstances like patient admission to the hospital or non-compulsory reasons such as failure to adhere to the treatment sessions. 
- The limited duration of "Off Period" required by ethical constraints of keeping a child off treatment

- The limited information gathered from the questionnaires that have only included answers to the closed end questions with no additional detailed information in the spaces provided for additional comments. 


\subsection{Conclusion}

The Bobath approach is an intervention that requires a continuous application to develop and maintain functional outcomes in children with CP. The most popular method of maintaining a continuous level of intervention is home treatment applied by caregivers. In an effort to promote home therapy adherence it is essential to engage the family in goal setting as well as a method of support and follow up. When assessing multiple methods of support, this study had indicated an educational course as well as a practical demonstration to be an effective method compared to an educational course accompanied by home visit follow-ups. the study had shown limited significant results, however, had shown some promising changes that might have shown more significant results if the study was extended to a longer period of time. The results were mainly limited by the small population of participants as well as the limited numbers of assessing therapists.

Results of this study could be further applied in an actual randomized controlled trial after the pilot study was found to be feasible. However, the results of this pilot study may further be applied in practice by concentrating on the education of the parents and caregivers regarding the effects of the home program in improving the functional level and maintaining results that have been obtained from previous physiotherapy courses. Follow up of adherence to the home program should also be applied to ensure continuity of home treatment which is a major factor in promoting the effectiveness of clinical physiotherapy treatment for children with CP. However, a less demanding methods should be used such as phone calls or text messages or even a diary. Simple, practical and cost-effective methods were found to be effective in previous research and must be considered to be applied compared to a weekly home visit method that did not show significant benefits in this study.

In order to further justify the results and personalize the findings a satisfaction questionnaire was needed. The questionnaire would provide a qualitative addition to the research that would support the level of evidence produced by the research. The questionnaire would also confirm the integrity of the trial and the level of consistency between the information provided to the parents regarding the procedures of the trial and the actual experience that they have had during the trial. 


\title{
Chapter 7
}

\section{Measuring the Level of Satisfaction of Parents with the Pilot Randomized Controlled}

\author{
Trial: A Survey
}

\subsection{Introduction}

As the previous chapter, had included a pilot study of the evaluation of the parents' level of support to the management of children with $\mathrm{CP}$, this study was established to evaluate the level of satisfaction that the parents have had from being part of the previous pilot study. The level of satisfaction was required to further identify any aspect that might or might not have been applied in the pilot study that might not have fulfilled the parents' goals or expectations, whether in the level of physiotherapy services or the stages of the pilot study. In populations that have difficulty in expressing their views such as children or elderly patients' views of close family or caregivers takes on the great importance (Ygge \& Arnetz, 2001). Many studies in the literature had assessed the satisfaction of parents with the services provided for their children (Avis et al., 1995, Ygge \& Arnetz, 2001). Such studies have indicated the importance of satisfaction of caregivers with the compliance with medical regimens. Evidence has found that information and knowledge regarding medical interventions lead to confident parents who in turn reflect these reactions to their children to feel more secure (Avis, 1995). In contrary parents who feel anxiety regarding their treatment intervention do reflect these feelings to their children leading to fearful and anxious patients (Wharton et al., 1996, Ygge \& Arnetz, 2001). Anxiety has also been found to affect the satisfaction levels and ratings of quality of care (Ygge \& Arnetz, 2001). A lack in the literature explored in chapter three and four was found in evaluating different aspects of parents' satisfaction in broader perspectives and results were only seen in evaluating pain management and medical encounters urging the need for a reliable and valid parent satisfaction instrument (Arnetz, 1999).

Consumer satisfaction in general and patient satisfaction is an inexpensive and efficient method of assessing the quality of services from the service users' perspective (Edlund et al., 2003). Moreover, the satisfaction of patients provides an input in health care decision-making in addition to obtaining information regarding the parents' perception of a 
program; therefore, it is essential to include patient satisfaction in the research of evaluation of services and interventions provided in the healthcare systems (Marino \& Marino, 2000; Weisz et al., 2005). Garland et al (2007) suggest that a limited amount of evidence has been found in the literature assessing patient satisfaction in addition to the lack of theoretical models guiding the investigation of satisfaction construct. Furthermore, Bickman (2000) indicated that satisfaction studies may be the most valid and simple instrument indicating quality compared with prolonged complex repeated measures of clinically significant change. Although Garland et al (2000) in agreement with Ygge and Arnetz (2001) had concluded a correlation between the severity of the cases of patients and their need of care with the level of satisfaction shown in the questionnaire and vice versa. Studies in the literature also lack consistency resulting in difficulty in making comparisons between studies. In addition, the inconsistent studies in the literature provide results that are difficult to estimate and therefore apply (Marino \& Marino, 2000). In 2000 Moumtzoglou and colleagues had presented a study to develop a valid and reliable satisfaction questionnaire that would assess the satisfaction level of parents of children receiving medical treatment bearing in mind the three distinct communication styles.

The three styles were created to be included in the practice of paediatricians that included:

- Informativeness: referring to both the quantity and quality of the information given to parents.

- Interpersonal sensitivity: attention and interest shown by effective behaviours

- Partnership building: the extent to which the physician would obtain parents; participation, opinions and suggestions.

As for services providing care of children with a disability, including physiotherapy led services to support children with $\mathrm{CP}$, over the past 20 years these services have developed to ensure that the family-focused approach had been included within the service delivery model that has been informed by the three styles mentioned previously (Moumtzoglou et al, 2000). This type of approach has been emphasizes a partnership between parents and service providers that focuses on including the family within the decision making process in regards 
to the child's assessed needs and thus recognizing the expert importance of the parents' view of their children's health status and needs as part of any service (Law et al, 1997).

Rosenbaum and colleagues (2008) had indicated that to provide a family-centred service, it is essential that parents (as well as their children) are satisfied with the provided services in addition to having confidence in the service they are accessing will achieve positive outcomes for their children. Aspects that were found to be most important for parents were consistency and continuity of services in addition to the involvement of parents in the intervention of their children. Levels of satisfaction were also found to improve when information exchange was sufficient, and respective and supportive services were provided and the main aspect that was found to show low satisfaction in children rehabilitation was the access to care (Hasnat \& Graves, 2000; King et al., 2001). Satisfaction is the main factor that promotes adherence to treatment services and recommendations for a home application which in turn leads to fewer feelings of distress, depression and overall improved level of wellbeing (Law et al., 2011). The previous chapter had demonstrated that a more structured a dedicated level of support that is inclusive of parents in the delivery of Bobath treatment can potentially increase physical function. However, it is essential that this therapeutic effect remains maintained throughout treatment cycles to promote the buildup of functional development as indicated by the previous studies in this chapter and the clinical audit study in chapter six. This finally indicates the need to evaluate the level of satisfaction of parents involved in the pilot trial with the stages of the study including the educational course elements.

According to the search and information found in the literature, it was decided that a satisfaction study was essential as a part of this research to address the level of satisfaction of parents/caregivers that have participated in the trial. This was performed to indicate the level of quality, validity and care that was experienced by the parents/caregivers in the pilot trial study detailed in chapter six. 


\subsection{Aims and Objectives}

\subsubsection{Aim of Survey}

To explore the level of satisfaction with of parents/caregivers who have been a part of the trial.

\subsubsection{Study Objectives}

Study three is a questionnaire survey that aimed to assess the following objectives:

- The level of communication and involvement of the parents in the information and stages of the trial

- The level of which the parents have been in control regarding any decision involving the wellbeing of their child.

- The level in which the parents felt that they have been in control of the decisions regarding the interventions provided for their child.

- The level of benefit that the parents/caregivers had gained from the information and education course regarding their child's case and management.

- The level of adaptation to the new therapists and level of care and affection the parents have experienced.

- The level of anxiety felt towards the trial and intervention.

- The level of satisfaction regarding the parameters of the interventions.

- The level of adherence and commitment to the requirements of the trial.

- The integrity of the researcher and the trial's procedures.

- The participant satisfaction of being part of the trial.

- The dissatisfaction elements that may have occurred for future improvement and change.

\subsection{Questionnaire Development}

\subsubsection{Informal Testing}

The questionnaire was initially developed according to the researcher's base of professional experience in clinical services in addition to the information that was found to be 
essential in assessing satisfaction levels that were retrieved from the existing literature. The initial format of the questionnaire was refined by the supervisor of this research to improve and develop the depth of the questions in addition to the layout and format of questions being closed or open-ended questions.

Changes that were suggested by the supervisor included grammatical and academic style writing comments. In addition to changing the format from closed too open-ended and the other way around to ensure the correct amount of information is gathered from each question and to simplify the analysis procedures for these questions. Other changes had included adding additional answering options or removing middle answers between yes and no also to ensure the correct amount of information gathered and keeping the answers clear rather than being inconclusive middle answers. Keeping the questionnaire simple and concise also encourages the participants to complete the questionnaire in a suitable amount of time. Changes had also included adding a tab for specifying and justifying some answers that the participants were encouraged to provide. An option of "I don't know" was also added for some questions that may provide information regarding specific questions.

\subsubsection{Formal Testing}

The questionnaire was then presented to two other supervisors that were experienced in paediatric research in addition to clinical research to approve the questions and provide any suggestions that they may have to improve the level of validity and reliability.

\subsubsection{Pilot Study}

Following the procedure of testing the validity of the questionnaire, a minor sample was selected for further testing. This was to ensure that the questionnaire was feasible and included simple lay language that didn't include any medical terms that might not be simple to understand by parents that are included in the trial. Another reason would be the ability to complete the questionnaire without any complication and within the time limit that should not exceed 20 min time. This pilot aimed at identifying any complications or obstacles that may be faced with this questionnaire that could be dealt with prior to performing the main study. 
The issued that were considered with this pilot study included time consumed to complete the questionnaire, the distribution procedures, and procedures undertaken with non-respondents.

The sample had included five parents of children receiving physiotherapy in the clinic. The parents were initially approached by their personal therapist. Following their approval, they were approached by the researcher and asked to fill in the 4-page questionnaire that aimed to evaluate the level of satisfaction that they were experiencing with the services they were receiving. The respondents to the questionnaire remained anonymous although it could be that there was some pressure felt by the parents/caregivers to complete the questionnaire after being asked in person to do so.

After the questionnaire was given to the parents the researcher asked the participants to hand in their filled documents to their therapists as soon as they were done. The participants were also asked to mention on the back of the document the time needed to fill in the questionnaire and the wording that they might have found difficult to understanding. The participants were asked to return the questionnaire at their next appointment which should have been no longer than a week for all participants. Three of the participants had completed the questionnaire on spot and returned the document to their therapist. One participant had returned the questionnaire the following session and the final participant had returned the questionnaire one week following the distribution of the document after being reminded by their therapist via a text message one day prior to their next session a week after distribution. All five filled questionnaires were collected from the therapists by the researcher after 2 weeks of distribution.

The pilot study was performed to evaluate:

- The return rate and to identify a strategy for dealing with non-respondents.

- The number of questions that have been completed by the participants.

- The time range required to complete the questionnaire.

- The level of skills required to analyse the data collected.

- The comments returned from the participants.

The pilot study was performed to avoid any conflicts or complications that might arise during data collection that might hinder the procedures of the survey and/or lead to insufficient responses or information gathered. 
The respondent to the questionnaire had shown a $100 \%$ return rate from parents/caregivers of five children with cerebral palsy that have been receiving treatment in the centre for at least one year. One respondent that has been receiving services for four years for his/her child with diplegia which was the longest period between the others. The other participants included a participant receiving 13 months of treatment for a child also with diplegia and two participants receiving two years of treatment for their children that had diplegia and left hemiplegia. Finally, a participant with a child with diplegia receiving two years and two months of treatment. Only one participant was encouraged to return the questionnaire with a text message. All the other participants have returned the questionnaire within the first week of distribution. The range of time required to complete the questionnaire was 5-15 minutes compared to the max 25 minutes expected and suggested to the parents.

\subsection{Main Study}

After the pilot study was executed the main study was then ready to begin

\subsubsection{Distribution of Questionnaire}

The questionnaire was distributed in the PMR hospital to all the participants that took part in the trial. The satisfaction questionnaire (Appendix 12) was distributed by the researcher following the end of the trial. After the final assessment was performed the parents/caregivers were approached by the researcher and provided the questionnaire after explaining the purpose behind it. Again, the participants were reminded that they were not obligated to fill in the questionnaire; however, it was mentioned that it was an important part of the study that they have decided to take part in. It was suggested that the questionnaire was to be filled at the clinic as it would require a maximum time of 20 minutes to complete. If the parents/caregivers were in a hurry to leave it was suggested that the questionnaire is handed back to their personal therapist in a week time. All participants except one have decided to complete the questionnaire on the spot as it only required a few minutes to complete. The single participant that decided to complete the questionnaire at home was sent a text message remainder after exceeding the 1-week time limit. The parents have returned the questionnaire the questionnaire the following week who referred the delay to their child not feeling well 
and did not seem fit for a session the prior week which caused them to be away from the centre more than a week and thus delaying the submission of the questionnaire.

\subsubsection{Analysis}

Data were analysed for this study that included the quantitative data retrieved from the closed questions within the pilot study and the main study and was explored using descriptive statistics and was displayed graphically.

As for open questions, responses within the questionnaire were considered to be qualitative data in nature and were recorded and evaluated using iterative analysis. Comparison of responses was continuously made to generate themes for discussion later on in the research. The comparison had also provided a tool to avoid researcher bias in the early stages prior to analysis.

\subsubsection{Ethics}

Ethical approval of this study was sought from the ministry of health in Kuwait that had approved the study in 2013 (Appendix 4).

The School of Health Sciences Ethics Committee in Swansea University reviewed the study and approved it in Sep 2015 (Appendix 5).

No further approvals were required to gain from the NHS Research Ethics Committee in the UK as the study was applied in Kuwait. 


\subsection{Results}

The trial had initially included 33 children, four children have withdrawn from the study and therefore at the end of the trial to evaluate the level of satisfaction a questionnaire was distributed to the 29 participants that were remaining (Appendix 12). After the final measurement was taken, at the end of the six weeks off period the questionnaire was handed to parents/caregivers. The parents were allowed a maximum of one hour to fill in the questionnaire as the questionnaire was evaluated to need approximately 15-20 min to fill in. all the participants have filled in the questionnaire with less than $20 \mathrm{~min}$. The questionnaires were then gathered and results were analysed using SPSS.

All 29 participants have agreed at the beginning of the questionnaire that the procedures of the trial had been clearly explained to them after reading the information sheet. They have also all agreed that they were given complete freedom to decide whether to join the study or not. All the participants have also agreed that the researcher was open to answering all their inquiries and questions and that they were happy to take part in the study. There was also a complete agreement that they were given complete freedom to withdraw from the study at any time with no questions asked (Q1- Q5). 


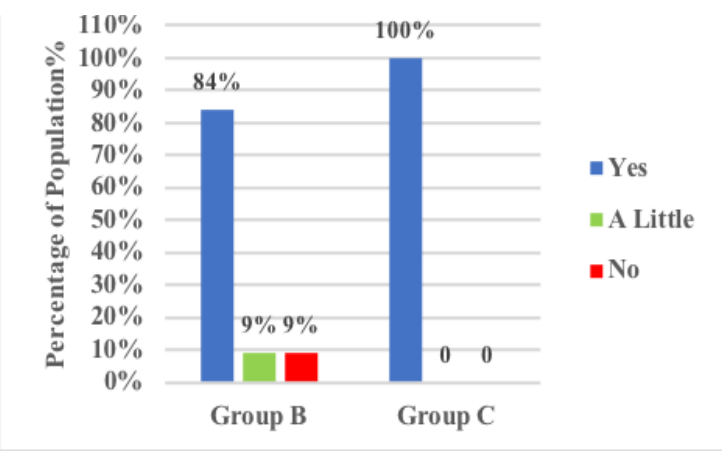

Figure 7.1 The Benefits Gained from the Educational Lecture Provided (Q6)

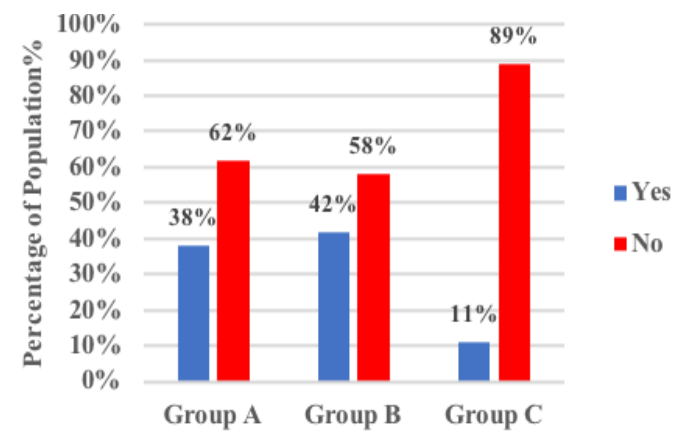

Figure 7.2 Did your child adapt quickly to the new therapist? (Q7)

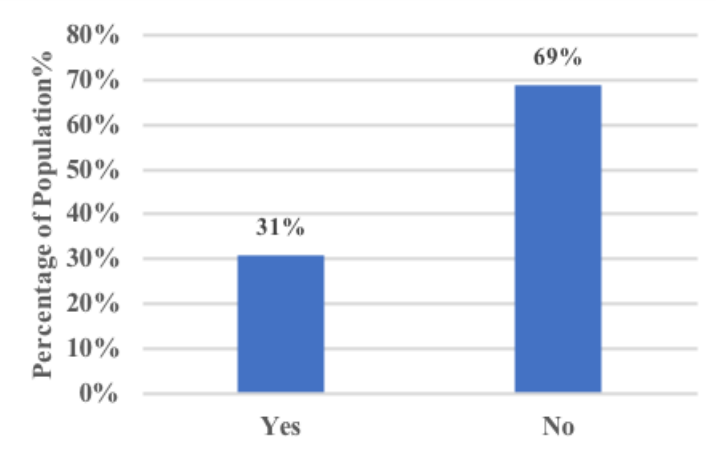

Figure 7.3 Did your child adapt quickly to the new therapist? (Q7). 


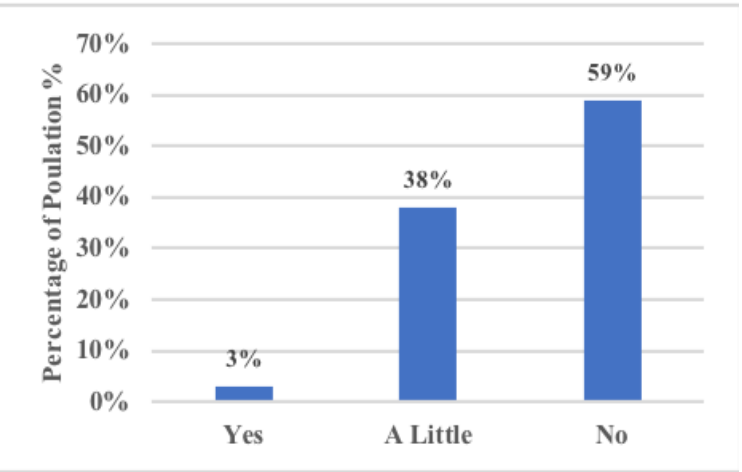

Figure 7.4 Did you have any doubt regarding the wellbeing of your child? (Q8).

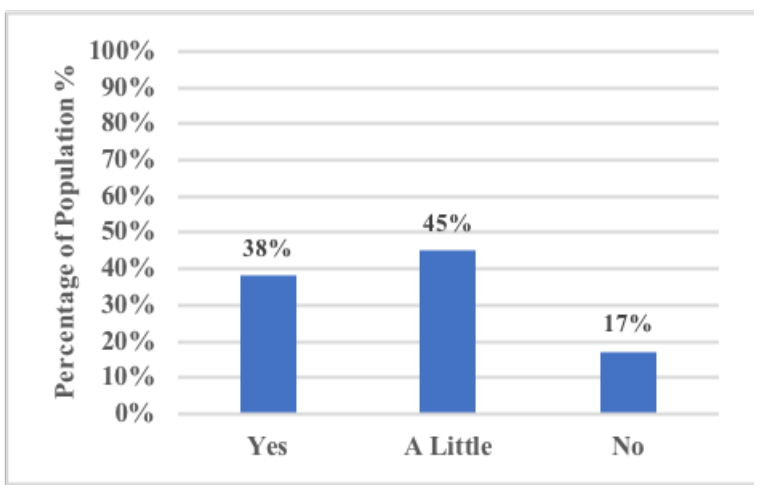

Figure 7.6 Was the frequency of treatment convenient to you? (Q9).

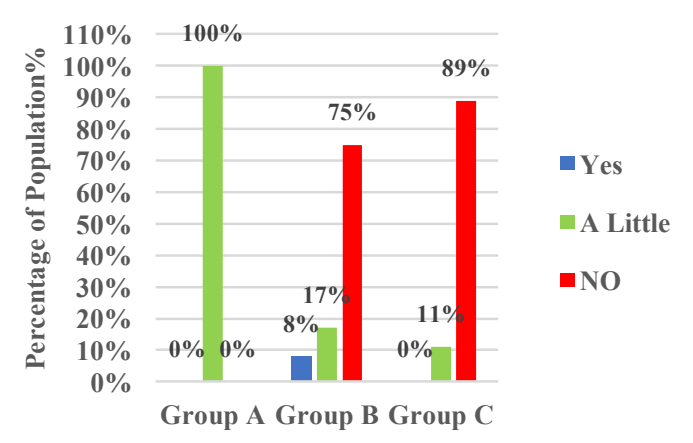

Figure 7.5 Did you have any doubt regarding the wellbeing of your child? (Q8).

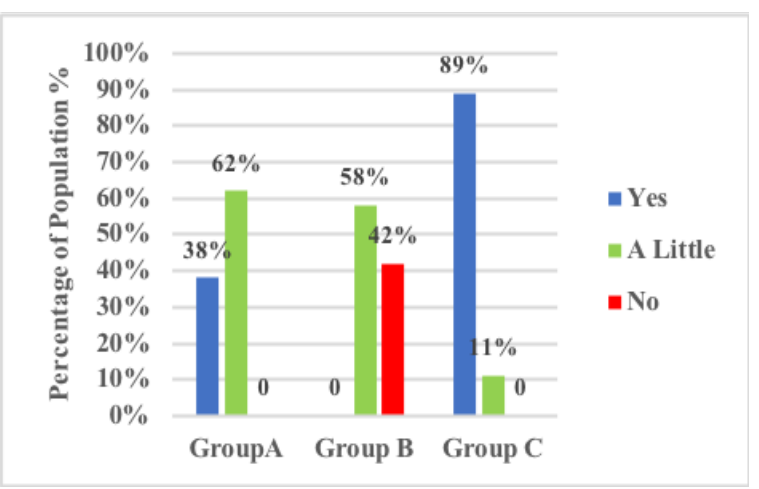

Figure 7.7 Was the frequency of treatment convenient to you? (Q9). 


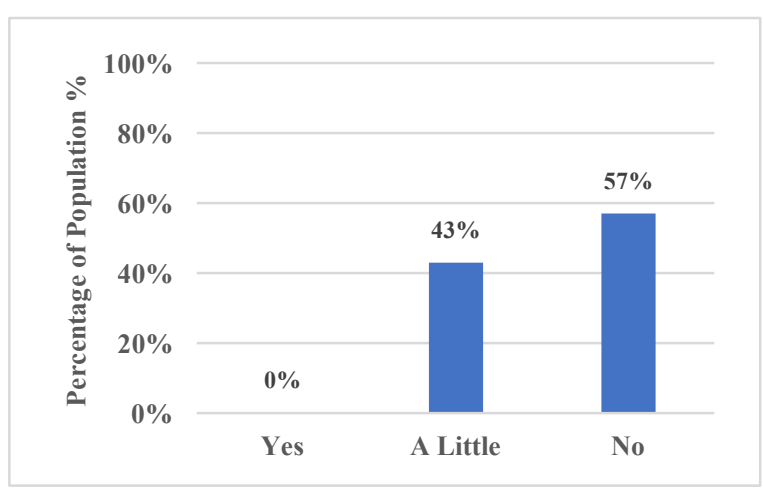

Figure 7.8 Was there any difficulty in coping with the frequency of the physiotherapy sessions? (Q10).

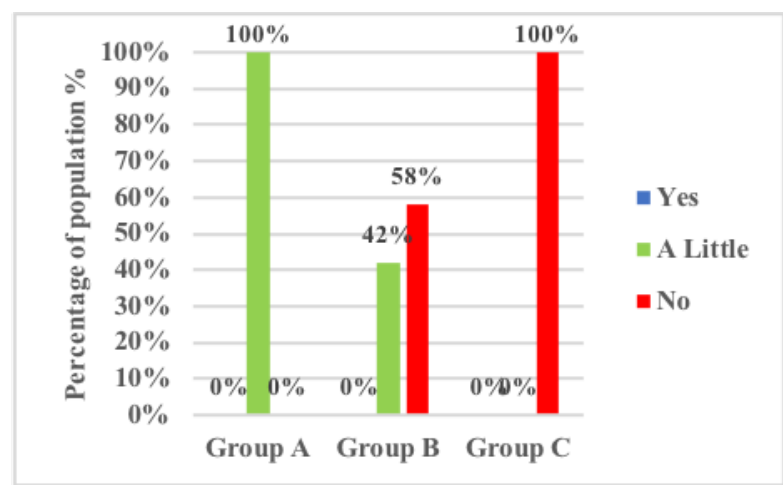

Figure 7.9 Was there any difficulty in coping with the frequency of the physiotherapy sessions? (Q10).

In response to the benefits gained from the educational course, more than $90 \%$ had agreed that they have gained benefit from the educational course leaving less than $10 \%$ indicating that they had little or no benefit at all (Fig. 7.1). The results unexpectedly showed all participants in group $\mathrm{C}$ to indicate gaining benefit from the "Educational Course" even though they did not show the highest level of change in GMFM results. This, therefore, may indicate that the benefits were not translated into more adherence to the home treatment however other benefits may be expanded knowledge of the child's condition or other information that was discussed in the "Educational Course".

In figure 7.2 (Q7) results indicate the level of adaptation that the children have shown toward the physiotherapist that applied the GMFM. The physiotherapists assigned to perform the GMFM were not the treating physiotherapists for the children. The level of acceptance of the assessing physiotherapist was then evaluated. Results show that the majority of $69 \%$ have shown levels of unacceptance for the assessing physiotherapist. When assessing the results according to group (Fig 7.3) the majority that failed to adapt quickly was found in Group C (Home Visit Group) with 89\% followed by Group A (Observational Group) (62\%) and finally group B (Educational Group) (58\%) showing significant results of $(p<.041)$ that will further be shown later in the results. The duration in which the child did not adapt to the new assessing physiotherapist may have affected the level of improvement during the clinical management. If the child does not cooperate with the physiotherapist during the session the 
benefits of that session would be limited which means that the overall effective physiotherapy sessions are reduced and at the end not all the children would have received the same amount of beneficial duration of physiotherapy management.

When evaluating the level of concern that the parents/caregivers have had regarding their children's wellbeing results (Fig 7.4) have shown that a high number of $38 \%$ had reported having little doubts whereas only $3 \%$ only which is equivalent to one person has had an actual concern. As for the other 59\% they have expressed having no concern at all. When assessing the results according to the groups, (Fig 7.5) shows that all participants in group A (Observational Group) have had some concerns while the only person that had clear doubts was allocated in Group B (Educational Group). The results are significant with a p-value of $(p<.001)$ that will further be shown later in the results. The level of concern was expected to reflect the personality of the families with regards to being overprotective or not.

More than $45 \%$ have reported that the frequency of the physiotherapy sessions was not as convenient as they expected (Fig 7.6). Another 17\% did not find the frequency convenient at all. The percentage that was satisfied with the frequency was equivalent to $38 \%$. When analyzing the results by groups, responses showed that Group C (Home Visit Group) showed the highest rate of satisfaction followed by Group A (Observational Group). Group B (Educational Group) had none of the satisfied population (Fig 7.7). These readings reflect the parents' beliefs that more sessions are more beneficial 
As for the difficulty experienced in attending the treatment sessions, it was found that $100 \%$ of group A (Observational Group) had reported having some difficulty while $100 \%$ of group C (Home Visit Group) was found to have no difficulty in keeping up with the frequency of the sessions. In group B (Educational Group) 58\% were found not to have any trouble with attending the treatment sessions in the received frequency (Fig 7.9).

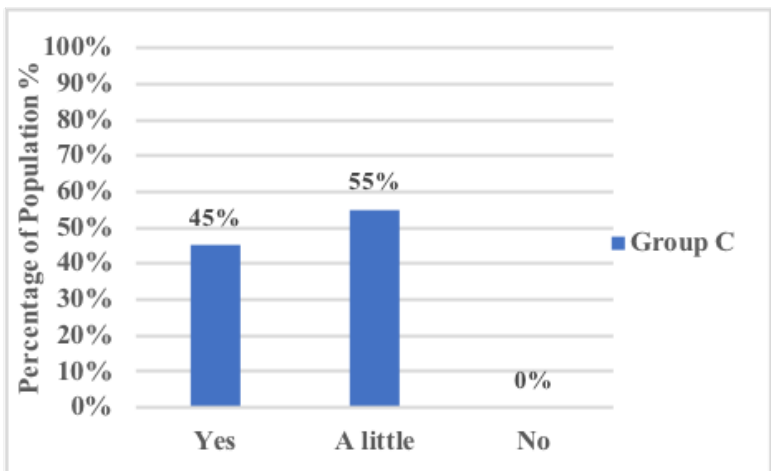

Figure 7.10 Were the home visits' frequency convenient to you? (Q11).

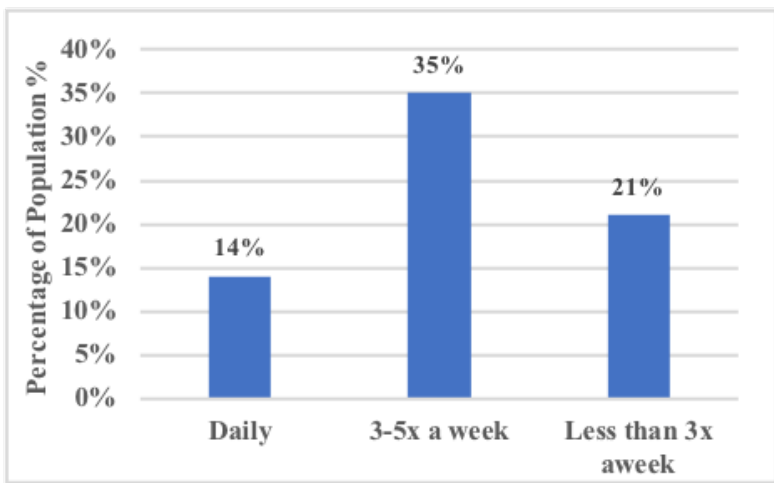

Figure 7.11 If you haven't received the home visit, how many times did you apply the home treatment at home? (Q13).

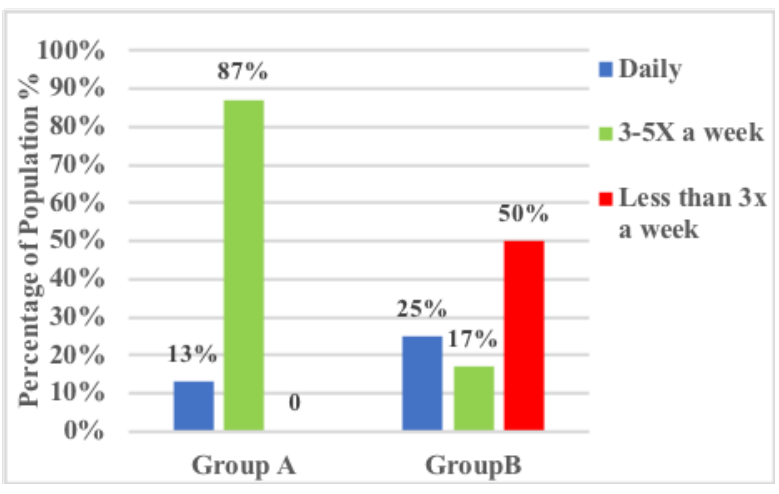

Figure 7.12 If you haven't received the home visit, how many times did you apply the home treatment at home? (Q13). 


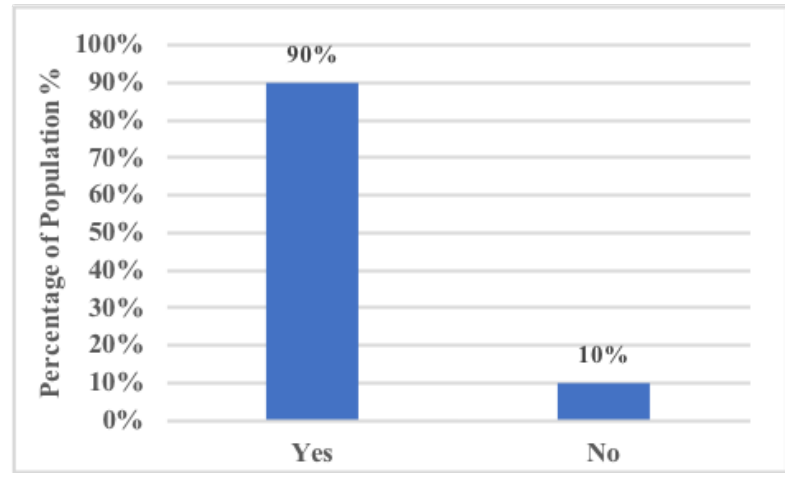

Figure 7.13 Was there any difference observed in your child's condition? (Q14).

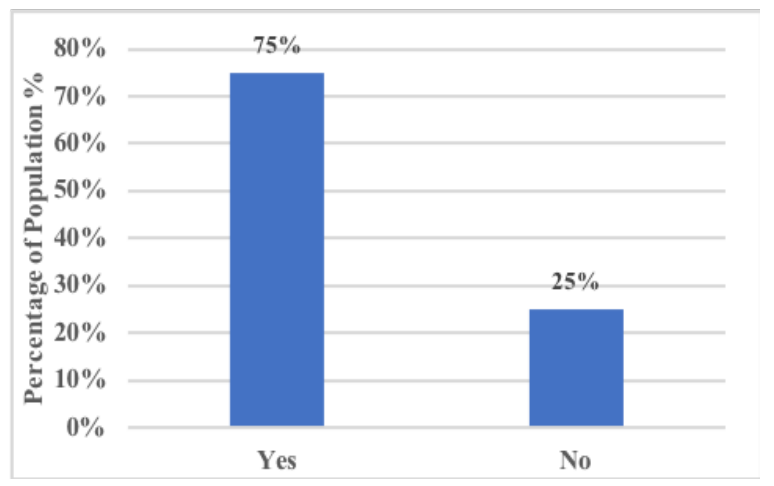

Figure 7.15 Were the procedures of the trial explained to you before taking part in the trial the same as what you have experienced in the trial? (Q15).

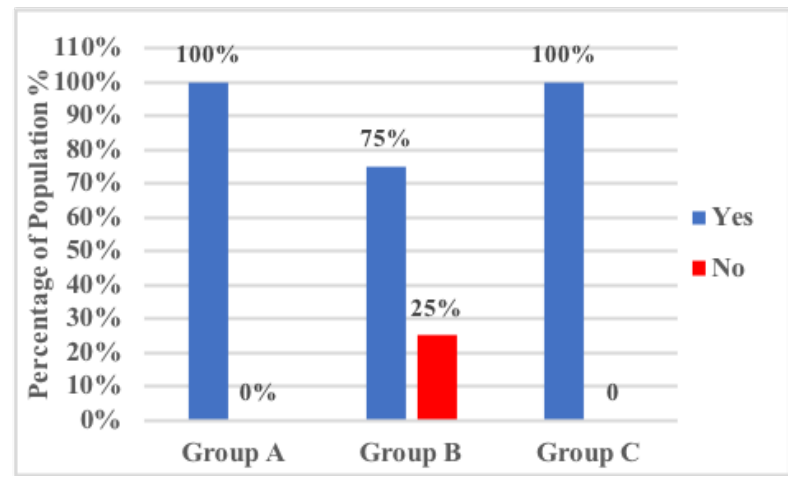

Figure 7.14 Was there any difference observed in your child's condition? (Q14).

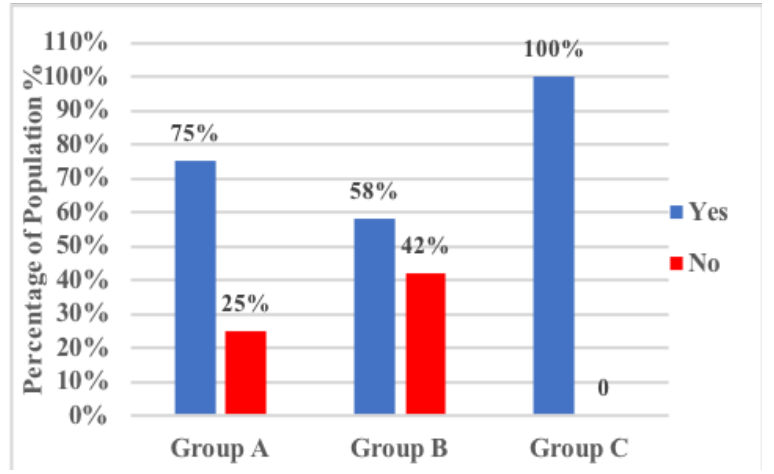

Figure 7.16 Were the procedures of the trial explained to you before taking part in the trial the same as what you have experienced in the trial? (Q15). 


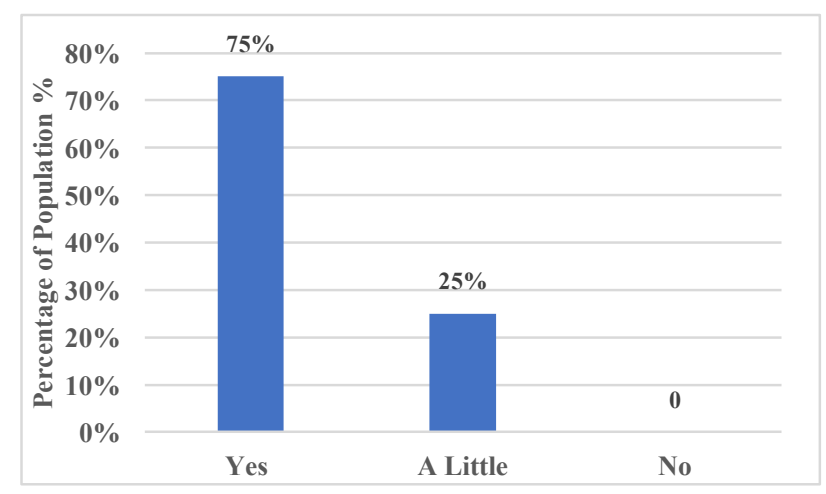

Figure 7.17 At the end of the trial, are you happy you took part? (Q16).

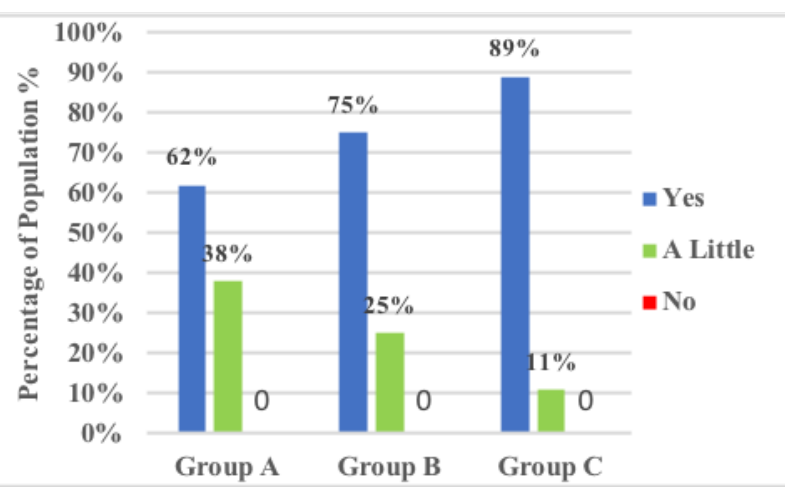

Figure 7.18 At the end of the trial, are you happy you took part? (Q16).

The response to the satisfaction of the frequency of the home visit group $\mathrm{C}$ was divided into satisfied and partially satisfied $45 \%$ and $55 \%$ respectively (Fig. 7.10).

All participants have indicated they have applied the home treatment (Q12). As for the frequency of the application $100 \%$ of group C (Home Visit Group) have reported applying the home treatment three to five times a week compared to $87 \%$ of Group A (Observational Group) that have applied treatment three to five times a week. As for group B (Educational Group), the majority of have applied the home treatment less than three times a week (Fig. 7.12).

In the differences that were seen in the children's progress $90 \%$ have reported finding a difference in the children's condition in figure 7.13. From this percentage, all the participants seen in figure 7.14 in groups C (Home Visit Group) and A (Observational Group) have reported seeing change, while in group B (Educational Group) only $75 \%$ have reported noticing a change in their children's conditions.

About $75 \%$ have agreed that the procedures explained at the beginning of the trial were the same as what they have experienced in the trial while $25 \%$ have reported the procedures not being the same (Fig. 7.15).

In figure 7.16 the groups show that $100 \%$ of group C (Home Visit Group) agree that the procedures were the same. On the other hand, in group B (Educational Group) $58.3 \%$ of the participants have agreed that the procedures were the same while $41.7 \%$ did not. In group 
A (Observational Group) $75 \%$ agreed that the procedures were the same compared to the remaining $25 \%$ that did not. The results are significant with a $p$-value of $(p<.005)$ that will further be shown later in the results.

In the final question, the participants were asked if at the end of the trial they were happy they had been part of the study and $75 \%$ have reported being happy while $25 \%$ reported being partially happy. No participants have reported not being happy with participating. With respect to groups group $\mathrm{C}$ have shown the highest level of satisfaction with $89 \%$ being completely happy with joining the trial (Fig. 7.17). The results are significant with a $p$-value of $(p<.005)$ that will further be shown later in the results.

As for the open questions of the questionnaire none of the participants gave answers to questions that requested further justification of the answers given or the final 2 questions that evaluated the reason that parents decided to take place in the pilot study or if the participants had any further comments.

\subsubsection{Comparison of Views Between Groups}

The views of the participating parents were furthermore compared depending on group allocation to evaluate if any significant difference is seen between the answers they reported in the questionnaire. A non-parametric group Cross Tabulation Chi-square test was performed to examine the relation between parents' views and the level of support provided in the different groups (A, B and C) in the pilot study.

Table 7.1 The comparison of views between groups.

\begin{tabular}{ccc}
\hline Groups & $\boldsymbol{N}$ & Sig $(\boldsymbol{p})$ \\
\hline A & 12 & .027 \\
B & 9 & \\
Total & 21 & \\
\hline
\end{tabular}

The relation between these variables was significant, $X^{2}(2, N=21)=12.629, p=.027$.

The results showed that parents in groups A and B had different opinions and views in the questionnaire regarding their experience (table 7.1). As expected the control group (A) would 
naturally have a different opinion regarding the procedures they went through compared to one of the experimental groups.

Table 7.3 The Comparison of views between groups A and C

\begin{tabular}{ccc}
\hline Groups & $\boldsymbol{N}$ & $\boldsymbol{S i g}(\boldsymbol{p})$ \\
\hline A & 12 & .022 \\
C & 8 & \\
Total & 20 & \\
\hline
\end{tabular}

When comparing the control group (A) with the other experimental group (C) the results were also significant, $\mathrm{X}^{2}(2, \mathrm{~N}=20)=11.429, \mathrm{p}=.022$ showing as expected the parents in groups $\mathrm{A}$ and $\mathrm{C}$ having significantly different opinions and views regarding their experience (table 7.2).

Table 7.2 The comparison of views between groups B and C

\begin{tabular}{ccc}
\hline Groups & $\boldsymbol{N}$ & Sig $(\boldsymbol{p})$ \\
\hline B & 9 & .012 \\
C & 8 & \\
Total & 17 & \\
\hline
\end{tabular}

When comparing experimental groups ( $\mathrm{B}$ and $\mathrm{C}$ ) the relationship between these variables was also found to be significant, $\mathrm{X}^{2}(2, \mathrm{~N}=17)=10.884, \mathrm{p}=.012$

Therefore, the parents in the experimental groups $\mathrm{B}$ and $\mathrm{C}$ were also considered to have different opinions and views in the questionnaire regarding their experience. As the two experimental groups underwent different variables the participants in the two groups were expected to have different opinions regarding their experiences. 
Table 7.4 The p-value of comparing responses between groups for each question.

\begin{tabular}{|c|c|c|c|c|c|c|c|c|c|c|}
\hline & $\begin{array}{l}\text { Q6) } \\
\text { Benefits } \\
\text { from } \\
\text { Edu } \\
\text { Course }\end{array}$ & $\begin{array}{l}\text { Q7) } \\
\text { Adaptation } \\
\text { to physio }\end{array}$ & $\begin{array}{l}\text { Q8) } \\
\text { Doubt of } \\
\text { wellbeing } \\
\text { of the } \\
\text { child }\end{array}$ & $\begin{array}{l}\text { Q9) } \\
\text { Convenience } \\
\text { with } \\
\text { physiotherapy } \\
\text { frequency }\end{array}$ & $\begin{array}{l}\text { Q10) } \\
\text { Difficulty } \\
\text { of coping } \\
\text { with } \\
\text { physio } \\
\text { frequency }\end{array}$ & $\begin{array}{l}\text { Q11) } \\
\text { Convenience } \\
\text { with home } \\
\text { visit } \\
\text { frequency }\end{array}$ & $\begin{array}{l}\text { Q12) } \\
\text { Frequency } \\
\text { of } \\
\text { applying } \\
\text { home } \\
\text { treatment }\end{array}$ & $\begin{array}{l}\text { Q13) } \\
\text { Progress } \\
\text { due to } \\
\text { home } \\
\text { treatment }\end{array}$ & $\begin{array}{l}\text { Q14) } \\
\text { Information } \\
\text { of trial } \\
\text { matches } \\
\text { stages of the } \\
\text { trial }\end{array}$ & $\begin{array}{l}\text { Q15) } \\
\text { Level o } \\
\text { satisfac } \\
\text { of joini } \\
\text { the trial }\end{array}$ \\
\hline $\mathrm{df}$ & 2 & 1 & 2 & 2 & 1 & 1 & 2 & 1 & 1 & 1 \\
\hline $\begin{array}{l}\text { Sig. } \\
\text { (p) }\end{array}$ & .000 & .041 & .001 & .166 & .450 & 1.000 & .247 & .000 & .005 & .005 \\
\hline
\end{tabular}

A non-parametric chi-square test indicates there was the significant difference in the proportion of groups about questions responses in group A in the current sample as compared with the value of groups B and C. In the above table, the group's responses are significantly different in which significant value is less than .05 .

The responses of the rest of the questions (Q1, Q2, Q3, Q4, Q5, Q14) were not included in the analysis as they all had agreed on the same answer (there was no variation) therefore, a chi-square test cannot be performed on these questions.

In the results of Q14 and 15 showed a significant difference between the control group (A) and the experimental groups $(\mathrm{B}, \mathrm{C})$ as expected. The results were predicted when the control group learned at the beginning of the study that their might be further education and home visits when taking part in the trial only to find out that they were the group not receiving all interventions of the trial. Therefore, the difference between groups was expected in these aspects specifically. 


\subsection{Discussion}

The satisfaction questionnaire was designed to evaluate the level of satisfaction that the parents/caregivers have experienced during the trial. It was also created to measure the level in which the parents/caregivers were happy to be part of the trial depending on more than one factor. In addition to the satisfaction regarding the integrity of the researcher and the procedures, regarding the wellbeing of the children, and the benefits that they have gained from being part of the study.

The Objectives of the study included:

- The level of communication and involvement of the parents in the information and stages of the trial

- The level of which the parents have been in control regarding any decision involving the wellbeing of their child.

- The level in which the parents felt that they have been in control of the decisions regarding the interventions provided for their child.

- The level of benefit that the parents/caregivers had gained from the information and education course regarding their child's case and management.

- The level of adaptation to the new therapists and level of care and affection the parents have experienced.

- The level of anxiety felt towards the trial and intervention.

- The level of satisfaction regarding the parameters of the interventions.

- The level of adherence and commitment to the requirements of the trial.

- The integrity of the researcher and the trial's procedures.

- The participant satisfaction of being part of the trial.

- The dissatisfaction elements that may have occurred for future improvement and change.

- The comparison of the responses between the three groups of treatment.

The sequence of the discussion will be according to the order of these objectives. 


\subsubsection{Level of Successful Communication Between the Parents and Researcher}

At the beginning of the questionnaire, the questions were focused on the clarity of explanation of the procedures and steps of the trial that the participants would be involved in.

Results of this section of the questionnaire and the total agreement of all the participants in questions 1 to 5 had indicated a high level of ethical consideration to the wellbeing and satisfaction of the parents/caregivers in having complete freedom in more than one aspect. Starting from the decision of taking part and followed by the freedom of withdrawal even after agreeing to participate. Results also reflect the level of openness in providing maximal amounts of information regarding the procedures and what is expected from the participants and what they should expect to experience in order to ensure all participants understand what they are taking part in before the beginning of the trial. This had promoted a level of understanding to assist the participants in deciding whether (or not) to take part. Being clear in explaining the study and being open to all inquiries of the participants have also resulted in no incidents of withdrawals from the trial due to unclear reasons. In contrary, it had led to an excellent commitment from the participants to the procedures throughout the trial duration. Which is also confirmed by the total agreement of the participants as being happy that they were part of this study.

Responses to the educational course had reflected a high level of appreciation and approval with $100 \%$ of the home visit group indicating gaining benefits from attending the course while $83 \%$ of the educational group indicated benefits gained from attending. This agrees with the conclusions found in Basaran et al. (2014) regarding the importance of education and communication of the medical and management team with parents and caregivers. Information exchange promotes a trust relationship and a support to assist the parents to manage and develop through the stages of accepting and furthermore managing the cases that their children have which in a match with what was concluded in Piggot et al. (2002) and Basaran et al. (2014). According to this, the educational approach is considered a successful tool in engaging the families and caregivers to be more involved in the management and goal setting for the children with CP and must be included in any approach of management of children with CP to ensure the comprehensive entire approach of treating a child with $\mathrm{CP}$. Having the course given to a group had also resulted in a supportive aspect to the families by understanding that they are not alone that are facing the challenges of caring 
of a child with special needs that had induced the commitment to the course and appreciation to the material delivered.

However, all the participants that were assigned to the observational group who have not received any amount of support or reassurance by the educational course have reported some level of concern regarding the wellbeing of their children before or during the trial. This finding also confirms the importance of communication and information exchange between the parents/caregivers and healthcare providers in supporting parents to better understand what intervention is being delivered to the children, what is to expect from the intervention, and what is to be expected from the children and their parents/caregivers in return. These results further emphasize the supportive effect of the educational group that should be considered a must in any facility providing management of children with CP. An educational supporting group requires limited efforts and costs compared to the effects that it may produce on the families.

\subsubsection{Levels of satisfaction with the procedures of the Pilot Study}

Another aspect of the questionnaire was the level of adaptation of the child to the physiotherapist that had applied the GMFM and how well the children have responded to the functional movements required. Sixty-nine percent of the total population had reported that the children did not adapt quickly to the new physiotherapist which have affected the level of performance of the functions required leading to the scoring level that did not represent the normal functioning level of the child. This was expected to may have influenced the scores of the GMFM that were not found to be significant. Better performance from the children to a more familiar physiotherapist may have improved the scores and may have resulted in more significant change found at the end of the study. However, to ensure the reliability of the results blinding of the assessor was an important aspect of the trial. Perhaps extending the assessment session with a play session prior to taking measurements would have played a role in improving the cooperation of the child with the physiotherapist which may have enhanced performance and furthermore improved scores of GMFM.

Another finding from question seven was that the home visit group scored the highest level of slow adaptation showing a significant difference $(p<041)$ from the other groups to the new physiotherapist which was referred to both the assessing physiotherapist and the physiotherapist that have delivered the home program at home (researcher). This may have 
also hindered the amount of benefit that could have been gained from the home treatment if the child was more familiar with the physiotherapist. According to that, a home program delivered by a caregiver or parent would avoid the need for the child to waste treatment time to adapt to another new physiotherapist. This also emphasizes the importance of the educational course that should qualify the parents in providing higher quality management of the children with CP. Also indicating that a weekly home visit which was a new approach applied in this pilot study may not provide the expected results of improvement in motor function due to the previous reasons. Therefore, monitoring the home delivery of treatment by the parents is better done through a different method. Other methods that do not include a therapist that may not be familiar with the families and have not provided treatment to the children before such as a phone call, internet video call or a diary are more beneficial to avoid the lack of response of families and children at home.

The parents/caregivers were asked if they were satisfied with the intensity of the sessions regarding the frequency which was a minimum of 2 sessions per week with the session duration of at least one hour. Surprisingly the parents/caregivers have expressed a level of dissatisfaction of $17.2 \%$ and a little satisfaction equivalent to $44.8 \%$ which are high percentages compared to the $37.9 \%$ that were satisfied in the total population. Responses were not found to have significant differences between groups in satisfaction $(p<.166)$ and therefore indicating that responses between groups were not highly variant and that little satisfaction was expressed with the physiotherapy services in the clinic. Although when searching the literature some studies have considered 2 sessions a week as being intensive therapy while some have referred to this intensity as a regular amount of physiotherapy. Aside from the approach that has applied intensive daily physiotherapy for two to three weeks none of the studies found in the literature has applied higher frequencies of treatment.

In addition, it should be mentioned that some children that were included in the study were receiving three times a week or daily sessions throughout the six weeks of treatment. Therefore, the results that were found in this aspect of the questionnaire were found to be unexpected and surprising. When further analyzing the results into groups it was found that the children assigned to group C (Home Visit Group) showed the highest level of satisfaction of $88.9 \%$ compared to the two other groups due to receiving the home visits even though $11.1 \%$ have still shown little dissatisfaction. Group B has shown the least level of complete satisfaction with $0 \%$. Results may be justified to the disappointment of group B participants after reading the information sheet handed at the beginning of the study and further learning 
from the researcher that they might be receiving a home visit. Not receiving the home visit similar to group $\mathrm{C}$ led to being a disappointment and dissatisfied. This reflects the importance of standardization of the parameters of physiotherapy treatment delivered. In addition, this raises an issue of the parents understanding what should be expected from physiotherapy intervention for children with CP. This again emphasizes the need for further education for the families to focus on quality and consistency of physiotherapy treatment rather than higher intensity and frequency of physiotherapy sessions.

Furthermore, when analyzing the results of the next question in the questionnaire which indicates experiencing any difficulties in coping with the frequency of the physiotherapy treatment sessions the findings were controversial. Results have shown that more than $41 \%$ of the participants have expressed that they have experienced some difficulties in coping with the frequency of the sessions. Moreover, when the results were further analysed to show the groups $33 \%$ of group B have reported having some difficulties in attending the sessions even though they have expressed not being satisfied with the frequency delivered in the previous question. In group (A) on the other hand, $100 \%$ of the population had also reported having difficulty in attending the sessions while more than $62 \%$ of them were also not completely satisfied with the frequency delivered. If anything, these results would indicate it would be that it was hard to keep the parents/caregivers satisfied with the services delivered. The parents/caregivers will always tend to ask for higher intensities of hands-on physiotherapy treatment even though they might not be able to benefit from it. This is also due to not knowing that the best practice may not always mean longer periods of clinical physiotherapy treatment courses or higher intensity interventions, as reported by Law et al. (1997) and Tsorlakis et al. (2004). To cope with such issues with families it is important to focus on tailored physiotherapy intervention to match the needs of each child and the family. To the families that may face some challenges in attending clinical physiotherapy focus should be made on home care and monitoring of home delivery. This may also provide better recourses to focus on clinical physiotherapy to other families that are not yet ready to provide home management for their children and are seeking higher intensities of clinical physiotherapy and a higher level of therapist support.

When group $\mathrm{C}$ was asked if the home visit frequency was convenient for them results showed decreased levels of satisfaction in more than $55 \%$ of the group with the once a week home visit. Although this is a new approach in Kuwait (home visits were never applied before for parents and caregivers) compared to studies found in the literature (Taylor et al., 
2004; Wang et al, 2013) this pilot study provided the highest frequency of home visits other studies available had provided visits monthly or twice a month. In other studies, such as Blide et al, (2011); Katz-Leurer et al., (2009); Ketelaar et al., (2010); and Tang et al., (2011), home visits were not at all offered and other methods were used to follow up the home treatment that was expected to be applied by the parents/caregivers such as phone calls or diaries that were checked after the study was over. This also refers to the attitude that some parent/caregivers have towards the services provided and is in some cases difficult to satisfy. This could be due to cultural issues. In Kuwait, private home physiotherapy services are considered very popular and therefore the participants may have expected the home visits to be for applying the home treatment rather than support and supervision. In future studies, the frequency of home visits must be strongly confirmed in advance in order to limit the families' expectations to what is offered to remain realistic and avoid disappointment. In addition, to ensure the equality between groups the home visits may be provided for the other groups after the study is terminated.

Results showed differences seen in the child's condition in more than $89 \%$ of the population compared to only $10.3 \%$ that did not see any changes. The group that did not see any effect of the treatment were further found to be all in group B, scoring $25 \%$ compared to the $75 \%$ that did see a difference with their children despite being the group that have scored the highest levels of change in GMFM measurements in the trial compared to the other two groups. This again shows the contradiction between the opinions of the participants with the findings of the trial and the questionnaire. This indicates that some cases of children show the progress that is usually not appreciated by parents especially if they have unrealistic goals for the child which emphasizes the need of further education regarding the expectations that the parents should have for their children's conditions.

At the end of the trial, $75.9 \%$ agreed that the procedures of the pilot study had matched the information and explanation given at the beginning of the trial compared to the $24.1 \%$ that did not agree. This indicates a good level of integrity of the researcher in applying the stages of the pilot trial. However, the other $24.1 \%$ of participants that did not agree were found to be assigned to group A (Observational Group) or group B (Educational Group). These results could be referred to that the information sheet handed at the beginning of the trial had mentioned that depending on the group that the child would be assigned to the parents/caregivers may receive an educational course or home-visit or both or may not receive any of them. Given that the disagreement of participants was due to the 
disappointment of not receiving one or both mentioned interventions. However, it was not possible if this was the case to acquire the satisfaction of all the participants as per the methods of this trial assignment to these different groups was a must. Blinding of participants to the procedures provided to other participants in other groups is necessary for future studies to avoid conflict and disappointment among participants.

In the end, the majority of (75.9\%) have agreed to be completely happy to be part of the pilot study $(\mathrm{p}<.005)$. The most group that felt happy to take part in the study was the home visit group (C) which was expected given that this group had received all the interventions offered in the trial. 


\subsection{Conclusion}

The study had aimed to evaluate the level of satisfaction of the parents/caregivers of the children that have been included in the trial. All 29 participants were included in the study and were asked to fill in the questionnaire that had assessed their views of the trial. All participants have returned the filled questionnaire and achieved a 100\% return rate.

Results have shown a high satisfaction rate to the communication provided at the beginning of the study that had included a detailed explanation of the objectives and information of the trial. Participants were also highly satisfied with the complete freedom provided in making the decision of participating or withdrawing from the trial at any time with no explanation needed.

The parents/caregivers have shown a level of alert regarding the wellbeing of their children at the beginning of the trial especially when several children did not adapt very quickly to the new therapist that had applied the assessment. Most of the participants have shown an acceptable level of satisfaction with the treatment session frequency in addition to the home visit frequency for the home visit group. A high level of adherence to the learned home program was reported in the questionnaire in the groups that did not receive home visits. As for the changes that have been noticed surprisingly the home visit group had reported the least satisfaction compared to the 2 other groups. Finally, the participants have reported a high level of "Feeling Happy" to have taken part in the study.

\subsection{Limitations}

Limitations of the study had included:

- The level in which the answers had reflected true views and opinions of the assessed aspects, as the participants were known to the researcher and questionnaires were handed in person which may have affected the level of integrity that the participants have shown in replying to the questions.

- Requiring the participants to fill in the questionnaires on spot may also have affected the quality of the answers and adding additional qualitative answers rather than just answering the closed questions compared to if the participants were allowed to fill in the questionnaire at home which may have provided more freedom to consume longer durations to provide more information. 


\section{Chapter 8}

\section{The Evaluation of the Views of Physiotherapists on Bobath for Children with CP in Kuwait: A survey}

\subsection{Introduction}

After exploring the evidence regarding physiotherapy for children with $\mathrm{CP}$ it is important to understand the views and opinions of paediatric physiotherapists in Kuwait. Also, the clinical audit in chapter 4 had identified some limitations of home treatment that showed that there was often limited support provided for the parents to apply physiotherapy at home. This study was created to better understand the views and opinions of the physiotherapists in the departments, whether Bobath qualified or not, regarding the best evidence-based practice and how to improve available interventions.

The Bobath approach is taught to qualified physiotherapists in private training courses that provides certified levels of training (DeGangi \& Royeen, 1994). Not all physiotherapists are Bobath qualified and other treatment interventions and principles are being applied (DeGangi \& Royeen, 1994). The views of physiotherapists, whether Bobath trained or not, regarding the intervention for children with $\mathrm{CP}$ and the Bobath approach provides a general overview of the reputation of Bobath and its principles and effects in treatment, particularly in relation to the actual regimes being actively applied in the departments.

In paediatric physiotherapy, more than one treatment approach is available for the management of children with CP. The Bobath approach, on the other hand, is a widely-spread treatment intervention that is used around the world (Butler \& Darrah, 2001). In different countries, Bobath is applied differently in parameters and objectives (Butler \& Darrah, 2001). As the approach depends on the variation of the disabilities, age, mental abilities and sociopsychological state of the children receiving it, it is difficult to provide a pre-set protocol of treatment that should be applied to all children with CP (Knox \& Evans, 2002). However, the variation in application parameters of the Bobath approach had perhaps affected the level of effectiveness in promoting functional progress in children with $\mathrm{CP}$, especially in the early years of life. This is due to the lack of knowledge of the correct and most suitable parameters of application of the Bobath approach. 


\subsection{Aim of Survey}

To investigate the opinions of therapists of Bobath treatment principles and the importance of the home program in supporting its effects.

\subsection{Study Objectives}

This survey-based study aimed to assess the following objectives:

- The age range, qualification, and level of expertise that the included therapists have in the treatment of children with CP in Kuwait to shed light on the variation of opinion.

- The methods for measuring treatment outcomes and the level of their validity and reliability.

- The importance of the chosen tools in measuring treatment results in children with CP.

- Opinions on the popularity and importance of the Bobath approach in the management of the problems of children with CP.

- The opinions regarding the treatment principles of Bobath.

- The parameters of treatment sessions.

- The importance of the home program in providing support to the clinical treatment.

- Whether home programs are being offered as part of the physiotherapy regime and what methods are being used.

- The level of commitment of the caregivers to the home program, the effect of the home program according to the level of commitment, and the procedures that should be taken to improve such process.

\subsection{Development of Therapists Survey Questionnaire}

\subsubsection{Initial Questionnaire Survey Development}

The Survey method was used targeting all paediatric physiotherapists in Kuwait. This method allows the investigation of a large amount of opinions in a highly reliable and valid procedure.

The questionnaire was designed and delivered using the website Survey Monkey This site facilitates the easy design of online questionnaires, including the use of open and closed 
questions. It also provides the option for printed copies to be distributed manually. The questions were written to address most common issues discussed in the literature on the Bobath approach and from the researcher's own experience of clinical services and the gaps between literature and practice.

\subsubsection{Pilot Study}

A pilot study took place prior to the official study, aimed at eliminating any bias that may have occurred during the main study. The pilot study included a sample of five therapists, who were each required to complete the questionnaire either manually or online. The participants were then approached by a senior therapist in the department who informed them of the questionnaire and the need to complete it within three weeks. The participants were also required by the senior therapist to document the time required to finish the questionnaire (if completed manually) on paper or to report it to the senior therapist if completed online. The five participants were reminded by text message after the three weeks had passed and those who had failed to complete the questionnaires were given another three weeks to complete them. The time required to complete the questionnaire as reported by the therapists varied between five to ten min for the four participants who filled in the online version. The participant that filled in the manual version took approximately 15 minutes to complete the whole questionnaire. No difficulty in filling the surveys was reported by any of the therapists.

\subsubsection{Main Study}

Following the pilot study, the survey was ready to be distributed as the main study.

\subsubsection{Distribution of The Survey}

The questionnaire (Appendix 13) was distributed to paediatric physiotherapy departments in Kuwait. The head of departments was first approached to gain approval. Approval had already been obtained from the Ministry of Health in Kuwait before approaching departments and staff. All 4 departments in Kuwait were contacted and a meeting with the head of each department was arranged to discuss the most suitable delivery 
method for the questionnaire. The decision mostly depended on whether the department allowed internet access through the department computers and whether the staff had access to internet-connected computers. A paper version questionnaire was distributed where internet access computers were not. A senior staff member was asked to act as a link between staff and researcher in order not to place any pressure upon the therapists to complete the questionnaires in each department. The staff were given a period of three weeks to complete the survey and then a reminder SMS text was sent. An extra period of another three weeks was granted after the text message was given. When paper questionnaires were used a record of the number of questionnaires distributed was made to allow us to evaluate the level of participation and completion. Each questionnaire (either manual or computerised) had a cover page explaining the aim of the study and a consent form clearly indicating that the therapists were not obliged to take part in the study and that the identity of participants would remain anonymous. The manually filled questionnaires were picked up by the researcher after the final three weeks.

\subsubsection{Non-response strategy}

The participants were given an initial period of two weeks to complete the questionnaire. An additional two-week period was given following a reminder of completing the questionnaire via a text message. The questionnaires that were not completed after the second two weeks are over were considered to be non-respondents.

\subsubsection{Analysis.}

Data analysis was performed using descriptive statistics. Quantitative data were described as frequency distribution and shown in percentages. The qualitative data gathered from the survey was further analysed per the theme of the analysis section.

Data analysis was performed using SPSS (IBM SPSS Statistics, version 22); descriptive statistics were used to analyse the results of the questionnaire to evaluate the frequencies of the respondents. 


\subsubsection{Ethics}

Ethical approval of the study was sought from the Ministry of Health in Kuwait that approved the study in 2013 (Appendix 4).

The School of Health Sciences Ethics Committee at Swansea University reviewed the study and approved it in Sep 2015 (Appendix 5).

No further approvals were required to gain from the NHS Research Ethics Committee in the UK as the study was applied in Kuwait. 


\subsection{Results}

Paediatric physiotherapists in Kuwait were surveyed on their views and opinions regarding the Bobath approach and the benefits of including a home program with it. SixtyFour therapists filled in the survey, with a total of 44 respondents returned via "Survey Monkey" with only 30 having been completely filled giving a return rate of $46.8 \%$. A copy of the survey may be found in Appendix 13.

All 44 participants confirmed in the survey's consent form that they had read the information sheet carefully, they had the freedom to withdraw at any time from the study without any questions being asked. They also confirmed that they were above 18 years of age and that they were fully qualified physiotherapists (Q1).

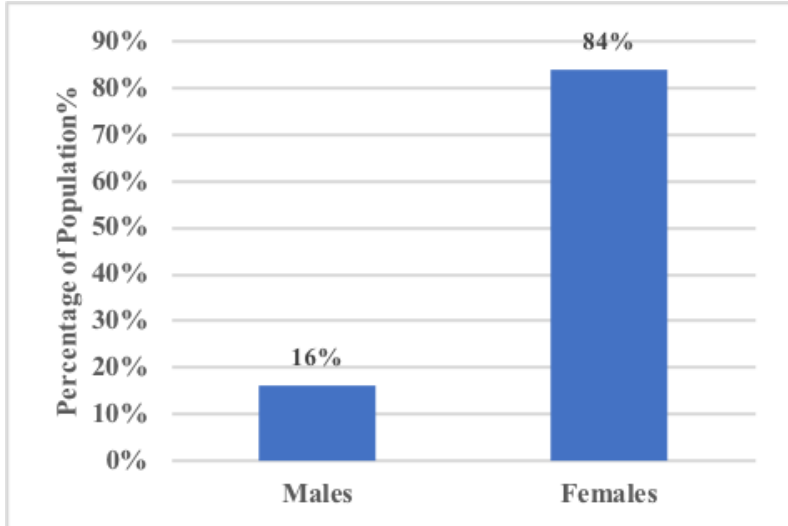

Figure. 8.1. What is your gender? (Q2)

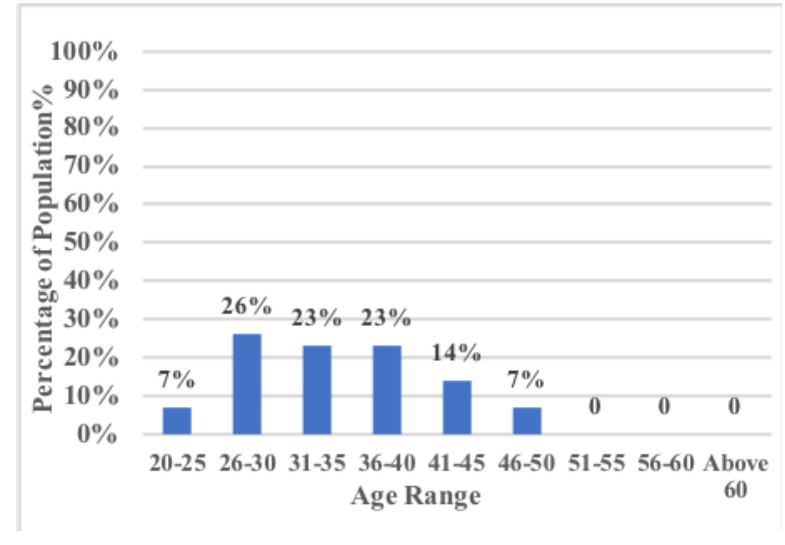

Figure. 8.2. What is your age range? (Q3) 


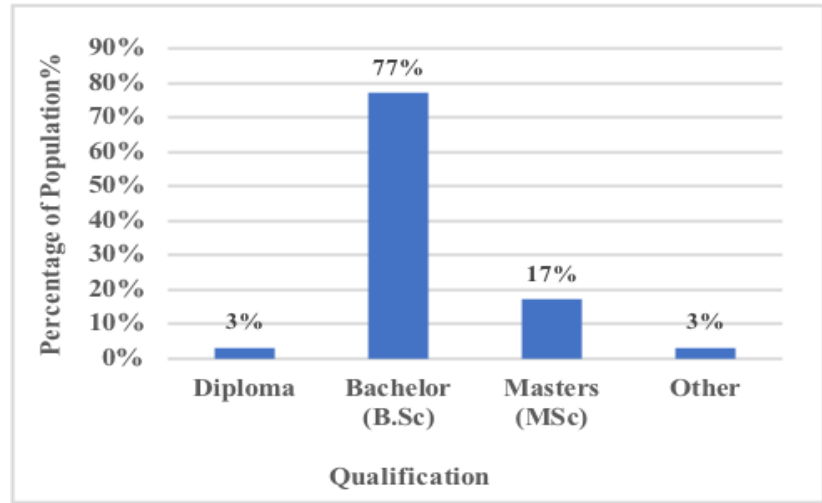

Figure. 8.3. What is your highest qualification? (Q4)

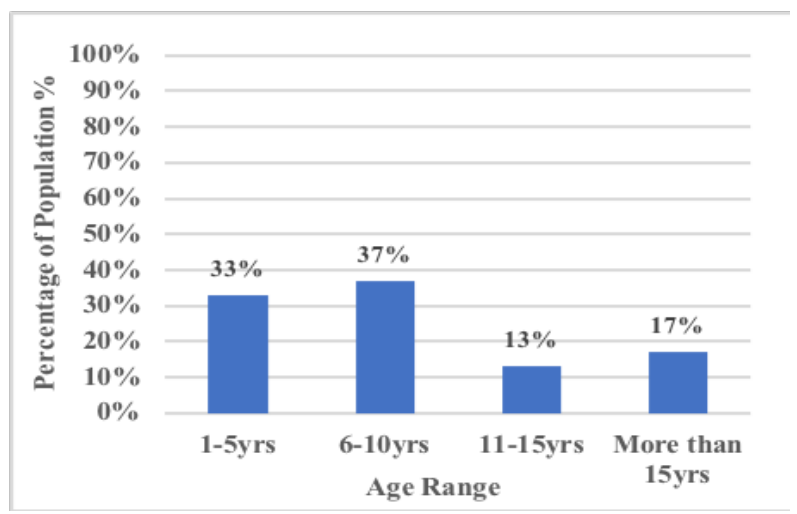

Figure. 8.5. How many years of experience do you have working in your current department? (Q6)

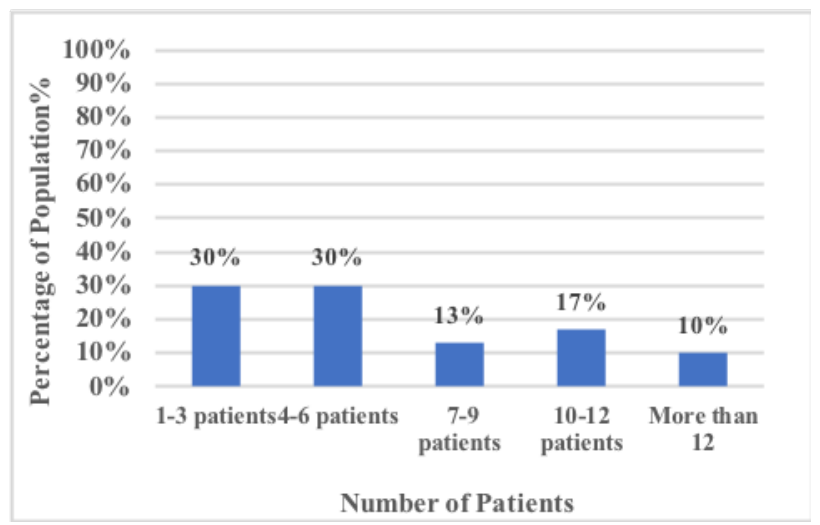

Figure. 8.7. How many cases of children presenting with CP do you see on average per week? (Q8)

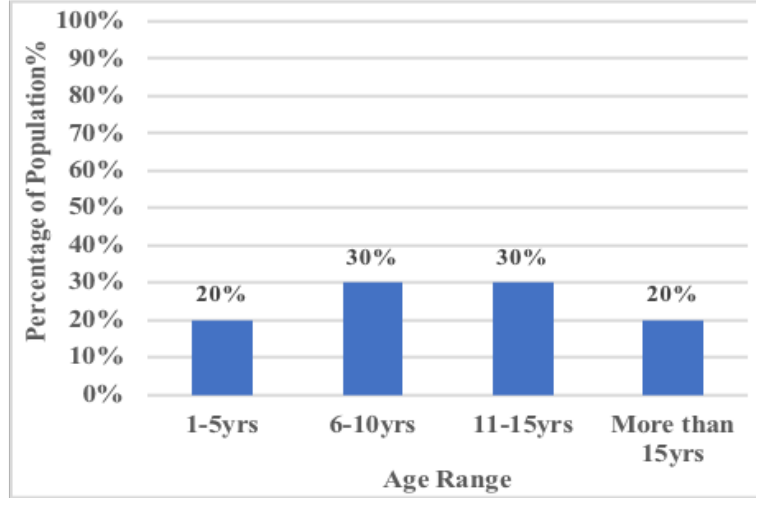

Figure. 8.4. How many years of experience do you have as a paediatric physiotherapist? (Q5)

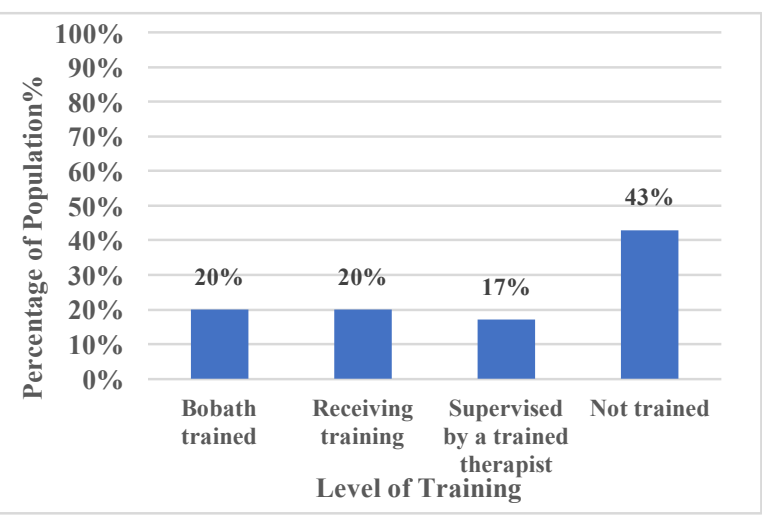

Figure. 8.6. What is your level of Bobath training? (Q7)

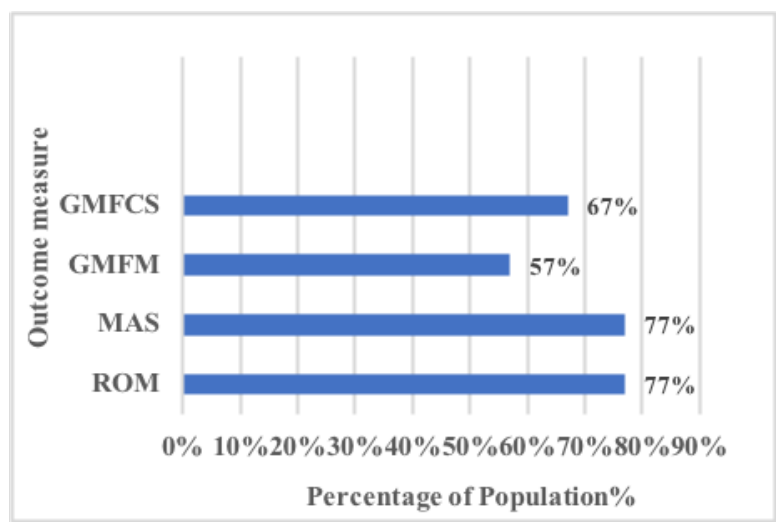

Figure. 8.8. What outcome measures do you use for assessing the patient's physical function? (Q9) 
In response to the second question, more than $84 \%$ (36/44) of the participants were 'Female' compared to the remaining 16\% (8/44) who were 'Male' (Fig 8.1). As for the age of the participating therapists, the range had varied between 20 and 50 .

The physiotherapist's qualification level was mainly bachelor degree with a total percentage of $77 \%$ (34/44) compared to the MSc degree holders reached 17\% (8/44) and diploma holders which had included one therapist only (3\%) (1/44). Only one other therapist had mentioned having had attended workshops qualifications but had failed to mention the techniques and interventions that he is now qualified in (fig 8.3).

The years of experience between the participants showed close values in figure (8.4). Fig 8.5 shows the years of clinical experience that the therapists have in the current department that they are working. Results show that $33 \%(15 / 44)$ have one to five years of experience while 37\% (16/44) have reported having experience between 6 and 10 years in the department. About 13 (5/44) indicated experience between 11 and 15 years while 17\% (8/44) have reported to have more than 15 years of experience in their current department.

The next aspect had evaluated the level of Bobath training. Nine of the participants $(20 \%)$ were Bobath trained and 9 participants were receiving training. Seven participants (17\%) reported being supervised by a Bobath therapist and were providing Bobath influenced treatment (Fig 8.6).

Fig 8.7 results show the number of cases of CP seen by each therapist per week. Results show that $30 \%$ of the population were seen between one and three patients with CP per week and another $30 \%$ were providing treatment for about four to six patients per week. More than $13 \%$ were seeing seven to nine patients a week while $17 \%$ were seeing about 10 12 patients. Some therapists reported seeing more than 12 patients every week which only represented $10 \%$ of the participating population. 
Fig 8.8 represented the outcome measure tools as reported by the therapists. The range of motion (ROM) and the Modified Ashworth Scale (MAS) had both scored a similar rate of preference with a percentage of $77 \%(34 / 44)$. These scores were followed by the GMFCS in the third place with a percentage of 67\% (30/44) followed by the GMFM with a percentage of $57 \%(25 / 44)$. Only one therapist selected the 'Other' option indicating the 'Normal Child Milestones' as an additional outcome measure tool to be added to the rest of the preferred measurement tools.

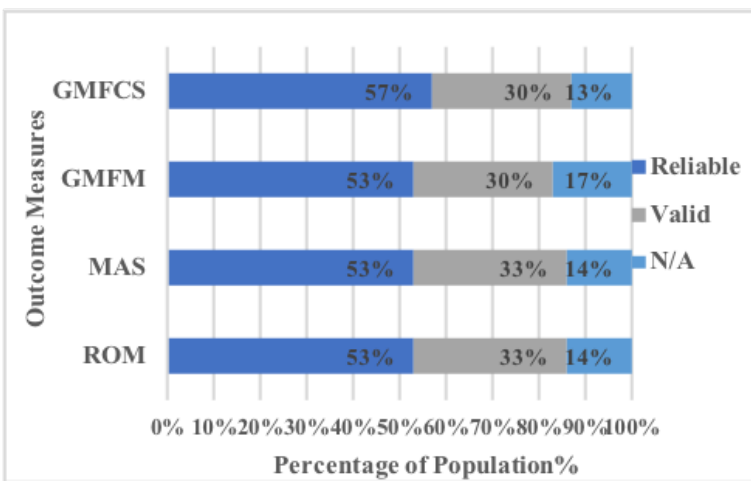

Figure. 8.9. Please indicate which of the following outcome measures do you believe has research evidence to suggest reliability and validity in assessing physical function in children with CP? (Q10)

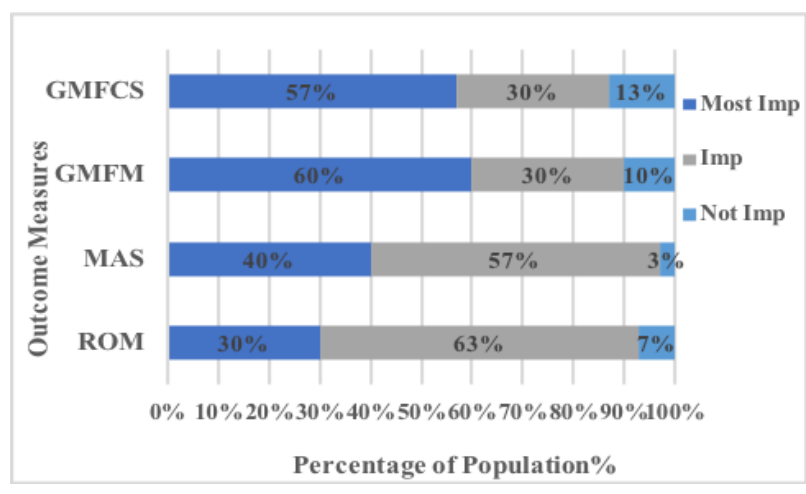

Figure. 8.10. Please indicate which of the following outcome measures do you believe has the most clinical value in assessing the outcome of treatment in children with CP? (Q11)

When the therapists were asked to evaluate the measurement tools that are most reliable to them the results showed that with respect to reliability the GMFCS scored the highest level of reliability compared to the other classification tools with a percentage of $57 \%$ (25/44). As the ROM, MAS, and the GMFM they showed equal reliability percentages of $53 \%(24 / 44)(\mathrm{Q} 10)$.

As for the validity of the measurement tools scores indicated the validity levels to be highest in ROM and MAS (33\%) (15/44) compared to the GMFCS and the GMFM that came next with a percentage of $30 \%(13 / 44)$ (Fig8.9).

When the physiotherapists were asked to evaluate the tools with respect to the level of importance in the assessment of children with CP results in figure 8.10 indicated that the GMFM scored the highest in the "Most Important" category compared to all the other tools with a percentage of $60 \%$ (27/44) confirming the popularity of this measurement tool. 
The frequency of the treatment sessions was shown in figure 8.11 to be mostly twice a week in more than $63 \%(28 / 44)$ of the therapist population compared to $23 \%(11 / 44)$ that reported providing treatment sessions three times a week. About $20 \%$ of the population reported providing daily treatment for their patients while $17 \%$ indicated other choices of treatment frequency that highly depended on the patients' needs. Only two therapists reported delivering therapy once a week ( $7 \%)$.

When asked about the Bobath approach $80 \%(35 / 44)$ of the therapists have reported believing that Bobath is a widely-used approach compared to only 2 respondents that disagreed while $17 \%(8 / 44)$ reported not being aware if it was (Fig 8.12).

When asked about their opinion regarding whether all children with CP should be receiving Bobath or not only 53\% (24/44) agreed while only 20\% (9/44) did not, with 27\% $(12 / 44)$ saying they were not sure.

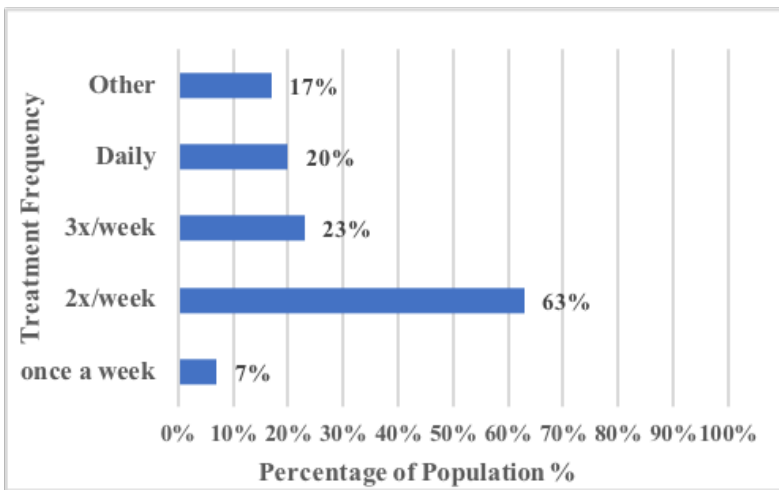

Figure. 8.11. For a child with CP how often would you normally provide a treatment session per week? (Q12)

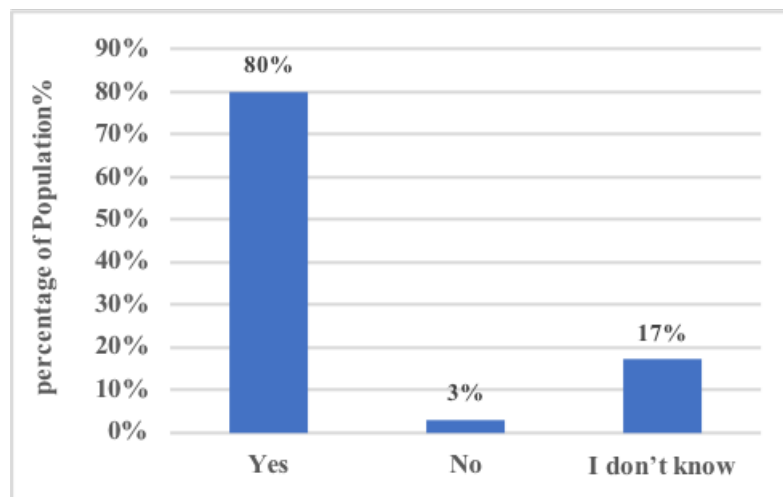

Figure. 8.12. Do you think that Bobath is a widely used approach? (Q13) 
With regard to the effectiveness of Bobath, $47 \%$ of the participants have indicated believing that Bobath was more effective than any other available approach whereas $23 \%$ did not agree. About 14 participants did not complete Q15.

In question 16 the responses of $63 \%(28 / 44)$ of therapists agreed that Bobath therapy should only be provided by therapists that have been trained and certified while $24 \%(11 / 44)$ of the therapists did not think the Bobath therapy should only be delivered by certified therapists.

Sixteen respondents $(37 \%)$ of the population had indicated that Bobath is not being delivered in the correct parameters in the departments. On the other hand, thirteen participants $(30 \%)$ were satisfied with the parameters delivered. The results of question 16 also showed the percentage of therapists that did not know the answer to this question had reached $33 \%$ of the participating population.

When asked about the principles of the Bobath approach $67 \%$ have agreed that Bobath was effective in inhibiting static and motor tone in children with CP. In question 19, $77 \%$ have agreed that Bobath principle of weight shifting was effective in improving posture while $70 \%$ of responses of question 20 agreed that the weight bearing principle was found effective in decreasing tone and increasing muscle power in children with $\mathrm{CP}$. Also, $67 \%$ have agreed to that Bobath is effective in inhibiting primitive reflexes that might be causing the delayed development of the child (Q21).

When asked about the effect of the home program in supporting the outcomes of the clinical treatment $74 \%(31 / 42)$ of the therapists agreed the home program provided major support to the Bobath approach. On the other hand, 12\% (5/42) of the population have reported believing that the home program only provided minor support to the main intervention and $14 \%(6 / 42)$ had indicated the moderate level of support that the home program provides to the clinical intervention (2 participants did not answer this question).

This was reflected in the following question (23) which had assessed if the therapists were providing their patients with home treatment and results have shown that $90 \%$ of the population has been providing home treatment. In this question, $10 \%$ of the participants did not provide an answer. 
In $60 \%(28 / 44)$ of the therapist population, the patients that have been provided with the home program have been reported to be partially committed to their home program while $17 \%(9 / 44)$ have reported their patients to be very committed to their home program. On the other hand, $13 \%$ (7/44) of therapists have reported that patients show no signs of commitment to their home treatment. As for the method by which the home program was being delivered, the therapists were using more than one method to provide the home treatment to the patients. However, the highest chosen method was the documented images with $73 \%$ of the cases. Practical demonstration, on the other hand, was preferred by $67 \%$ of the therapists compared to the verbal explanation that was reported to be used by $53 \%$ of the therapists.

Sixty seven percent of the therapists have agreed that the effects were seen as a result of applying for the home program while $20 \%$ have only seen some effect to an extent as a result of applying the home treatment at the same time $3 \%$ of the participants have reported not seeing any benefits on the prognosis of the children. Therapists reported in $80 \%$ of the cases the need of educating the caregivers regarding the importance of the home program in supporting the effects of the clinical intervention and, moreover, the prognosis of their children.

To assess whether the level of experience affected the choices that the therapists made analysis was performed to compare the responses of two groups that were created according to years of experience. Participants were divided into two groups 'Above 10 Years of Experience' and 'Less than 10 Years of Experience'. Physiotherapists that had experience up to 10 years were allocated to group A whereas therapists that had more than 10 years of experience were allocated to group B. Results were further analysed for questions 9, 10, 11, 12 and 25.

Table 8.1 Creating two comparison groups depending on the years of experience.

\begin{tabular}{|c|c|c|}
\hline Groups & Category & N \\
\hline A & Physiotherapists less than 10 years of experience & 15 \\
\hline B & Physiotherapists above 10 years of experience & 15 \\
\hline
\end{tabular}


Chi-square test of independence was performed to examine the relation between the views of group A and group B about the choice of measurement tool (Q9). The difference between the choices of group A and B was not significant ( $p=0.880,0.880,0.893,0.239$ ).

Table 8.2 Showing a comparison between groups A and B in the responses regarding the reliability and validity of ROM as a tool of measurement in Q10

\begin{tabular}{ccc}
\hline Q10 & Responses & p-value \\
\hline ROM & Reliable & 1 \\
\hline & Valid & 1 \\
\hline MAS & Reliable & 0.785 \\
\hline GMFM & Valid & 0.763 \\
\hline & Reliable & 0.789 \\
\hline GMFCS & Valid & 0.887 \\
\hline & Reliable & 0.891 \\
\hline & Valid & 0.880 \\
\hline
\end{tabular}

In table 8.2 a chi-square test of association was performed to examine the relationship between groups A and B in the evaluation of the ROM, MAS, GMFM and GMFCS. The difference between the views of groups A and group B were not significant.

A non-parametric cross tabulation chi-square test was applied performed to examine the relationship between groups A and B in the evaluation of the importance of ROM, MAS, GMFM and GMFCS in question 11. The difference between the views of groups $\mathrm{A}$ and group B were not significant in the ROM and GMFM categories ( $\mathrm{p}=0.839,0.687)$. The chisquare showed an error in the MAS measurement tool due to a value of zero in the results, therefore, a Fisher test was used to find the $(\mathrm{p}$-value $=1)$. The difference between group A and B was found significant $(p=0.0006)$ for the GMFCS classification tool. This indicates 
that the therapists with higher experience had a different evaluation of the importance of the GMFCS compared to the physiotherapists with less experience.

A Kruskal Wallis test was performed to examine the relationship between groups A and $\mathrm{B}$ in the frequency of treatment (Q12). The difference between the views of groups A and group B were not significant $(\mathrm{p}=1)$.

A non-parametric cross tabulation chi-square test was applied performed to examine the relationship between groups A and B in the method used by the physiotherapists to teach the families the home treatment (Q25). The difference between the views of groups A and group B were not significant $(\mathrm{p}=0.738,0.763,0.789)$.

Another comparison was made between the physiotherapists with respect to the level of Bobath training they had. Participants were divided into groups $C$ and D. Group C had included the physiotherapists that were Bobath trained or are under training while group D had included the physiotherapists that were only supervised by a Bobath therapist or are not trained. Results were further analysed for questions 9, 10, 11, 12 and 25.

Table 8.3 Two comparison groups with respect to Bobath training level.

\begin{tabular}{|l|l|l|}
\hline Group & N & Category \\
\hline Group C & 12 & Bobath Trained, Under Training \\
\hline Group D & 18 & Supervised by Trained Physiotherapist, Not trained \\
\hline
\end{tabular}

Chi-square test was performed to examine the relation between the views of group $\mathrm{C}$ and group D with regard to the choice of measurement tool (Q9). The difference between choices of group $\mathrm{C}$ and $\mathrm{D}$ is not significant in any of the measurement tools $(\mathrm{ROM}=0.8$, $\operatorname{MAS}=0.9, \mathrm{GMFM}=0.9, \mathrm{GMFCS}=1.0)$.

A non-parametric cross tabulation chi-square test was performed to examine the relationship between groups $\mathrm{C}$ and $\mathrm{D}$ in the evaluation of validity and reliability of measurement and classification tools (ROM, MAS, GMFM, GMFCS) in question 10. None of the results of the difference between the views of groups $\mathrm{C}$ and group $\mathrm{D}$ was significant.

Another chi-square test was performed in Q11 to examine the relation between groups $\mathrm{C}$ and $\mathrm{D}$ in the evaluation of the importance of ROM, MAS, GMFM, GMFCS in paediatric assessment. A significant difference was found between the two groups in the importance of 
MAS measurement tool $(\mathrm{p}=0.003)$. This had shown that the level of training had affected the opinions of the therapists regarding the importance of a measurement tool.

A Kruskal-Wallis test was applied to examine the relationship between groups $\mathrm{C}$ and $\mathrm{D}$ in the frequency of treatment. The difference between the views of groups $\mathrm{C}$ and group $\mathrm{D}$ were not significant $(\mathrm{p}=0.386)$.

Finally, a non-parametric cross tabulation chi-square test was performed to examine the relationship between groups A and B in the method used by the physiotherapists to teach the families the home treatment. The difference between the two groups was not significant ( $p$-value for the three categories was $(p=0.717,0.756,0.689)$. 


\subsection{Discussion}

The survey collected the views of the therapists regarding the Bobath approach depending on their years of experience, age and level of Bobath training.

\subsubsection{Information of the Personnel of the PMR Paediatric Department}

The level of experience of paediatric physiotherapists was found to vary widely within the paediatric physiotherapy departments in Kuwait. The duration in which the physiotherapists spent in the same department, on the other hand, showed that the staff continuously transferred from one hospital to the other. Also, it had indicated that many physiotherapists were foreigners working in Kuwait.

When investigating the level of Bobath training more than $56 \%$ of the participants indicated either being 'Trained', 'Receiving Training' or were 'Supervised by a Trained Therapist' and are providing Bobath influenced therapy compared to the $43.3 \%$ of staff that were 'Not Trained'. This indicated that Bobath is popular and is being widely used in all the departments in Kuwait. This agrees with most of the studies in the literature that have referred to Bobath as the most common approach for treating children with $\mathrm{CP}$.

\subsubsection{Popular Outcome Measures in Kuwait}

It was found that the most popular methods used in the assessment of children with $\mathrm{CP}$ were the range of motion and the Modified Ashworth Scale for measuring tone, with $76.7 \%$ of physiotherapists reporting they used each. Given that the majority of therapist has indicated the use of Bobath treatment it seems questionable that ROM is the most suitable measurement tool, especially since the Bobath approach does not deal with muscle length. Bobath mainly focusses on other aspects of tone, posture, and in some cases muscle power that affect the functional level and should be measured functionally by either the Wee FIM or the GMFM (Which is the reason that the GMFM was the outcome measure used in the pilot study in chapter six). According to Knox and Evans (2002) treatment for children with CP, whether or not the Bobath approach is being used, should be delivered as functional activities and movements. Therefore, it would be sensible to measure the change in the prognosis of children under treatment as functional outcomes, something which is confirmed in other 
studies, including Russel et al. (2002), Ahl et al. (2005), Han et al. (2010), Ko and Kim (2013). Therefore, indicating that the GMFM is the most appropriate outcome measure to be used. Results of the survey rank the GMFM as the least preferred choice of measurement tools and the Wee FIM, not at all preferred. This does not reflect the correct relation between the treatment objective and the choice outcome measure used by the physiotherapists (Fig 8.8). The GMFM has a very prolonged procedure of application that may exceed an hour to apply and also requires special training for the therapist to qualify to use the tool, however, it should not be neglected due to this negative aspect. It should not affect the need to assess change in functional abilities in children either especially that the tool can be applied to different methods that could be less time-consuming. It could also be used to focus on specific aspects of function that is expected to show a change in response to treatment rather than the total functional level of the child.

In the following aspect of the survey, the therapists chose ROM, MAS, and the GMFM to be equal in validity and reliability (fig. 8.9) and in (Q11) the therapists have chosen the GMFM to be the most important tool in measuring outcomes in children with CP (fig. 8.10). This contradicts their results of choosing ROM and MAS to be the most used tool in their applied assessment in question 9 (figure 8.8) even though they understand the importance and priority of the GMFM compared to the other tools for children with CP. This was also seen in terms of the Beery VMI test when the results of Q9 that indicated none of the therapists used this measure in the assessment of children with CP while in figure (Q11) the results had shown that more than $13 \%$ had indicated the Beery VMI test to be important or very important to them in the measurement of outcomes in children with CP. These findings indicate conflicts between the beliefs of the physiotherapists and the methods they use in practice, something DeGangi and Royeen (1994) observed in their study. They found that physiotherapists believed that NDT is a total management program of the child that they should consider the development of functions in a different environment. However, more than $50 \%$ of the same respondents did not think that progressing the child's functional level in different environments such as the home was an important aspect of the therapy. This situation is similar to what was found in the clinical audit in chapter six. Patients' records had revealed that the physiotherapists had believed in the importance of the home treatment and had provided a number of documented exercises. However, they have failed to provide any follow-up and update to the home treatment provided. 
Further analysis was thought to be helpful in finding a correlation between the choices of measurement tools and the level of training or experience. The correlation that was found between the level of experience and the importance of using the GMFCS $(p=0.0006)$ had provided relevance to the results. It showed that the physiotherapists with higher levels of experience had chosen the GMFCS to be a very important part of the management of children with CP while the group of physiotherapists did not think it was an important part of the treatment. GMFCS is the classification tool that is used to evaluate the level of severity of CP which would then help in the expectation of mobility levels and other symptoms which helps in goal setting and focus of intervention. Therefore, the experience of the physiotherapist shows to affect the plan of classifying the child with CP that is considered to be the key element of assessment and treatment (Chapter 1). Such results indicate the classification tool to be more popular with the older physiotherapists. This could mean that the older physiotherapists are following a protocol that depends on classifying the case of CP prior to starting the management plan and that the younger population of physiotherapists were not familiar with such a protocol. It could also mean that the younger physiotherapists do not depend on classifying the cases of CP depending on severity predicting the future symptoms that could be expected according to the level of GMFCS. However, they may focus on managing the symptoms that the children present in the assessment sessions

As for comparing the groups according to the level of Bobath training, the results showed significant differences in the importance of MAS measurement tool $(p=0.003)$ in favour of the physiotherapists with higher Bobath training. This justifies the choice of both groups. The choice made by the group with higher training in Bobath indicates a better understanding of the principles of Bobath that depends on limiting the tone in CP cases. While it might not be fully appreciated by the group of therapists that are not highly trained or not Bobath trained at all.

\subsubsection{Views of Bobath Treatment}

When being asked general questions regarding their opinions of the Bobath approach the therapists have indicated that it is very widely used around the world and that all children with CP should be receiving it. This was based on their belief that it is the most effective treatment approach for children with CP. Although it is considered to be the most widely known treatment intervention this has not been proven and most, if not all, studies that assess 
the effectiveness of the intervention are relatively outdated (older than 2006) and inconsistent in results or methods (Butler \& Darrah, 2001; Fetters \& Kluzik, 1996; Knox \& Evans, 2002). Responses indicating that all children should be receiving Bobath is considered inapplicable due to the lack of resources and the limited number of Bobath trained therapists and therefore an unrealistic objective according to the Bobath training level of the physiotherapists.

The survey revealed that more than half of the therapists either are trained, receiving training or are supervised by Bobath therapists. However, there are still more than $40 \%$ of therapists providing treatment for children with CP who are not at all Bobath trained. This percentage could be generalized to represent many of the paediatric therapists around the world that are either providing bobath influenced therapy or other therapies. Many paediatric therapists are not Bobath trained but continue to treat children with $\mathrm{CP}$ and continue to achieve good results. These therapists may be providing different types of handling and other treatment techniques that show to be effective in children with CP. This indicates that Bobath is not the only effective approach and that not all children with CP are in need for the Bobath approach even though many physiotherapists still strongly believe that it is the most effective therapy used. Another aspect would be that many therapists as part of their bachelor studies have learned the aspects and principles used for inhibiting tone and primitive reflexes in addition to correcting postures and many other functional exercises and preventive positions without them being referred to any identified approach. These therapists are actively and effectively applying these skills in treatment for children with CP and are effectively reaching set goals even though they are not officially Bobath trained. Therefore, the principles of Bobath may be learned in ways other than through the official training course. Moreover, if the Bobath approach was considered as the most important approach then perhaps it should be taught as part of the qualifying degree in physiotherapy schools rather than being offered as prolonged skill courses that may apply a financial burden to both therapists and health institutes not to mention the prolonged duration time commitment. However, the titles that are given to officially trained physiotherapists are not gained if trained unofficially in undergraduate levels or by seniors in the workplace. this makes all the difference in the physiotherapist curriculum vitae and therefore affects the career level and job opportunities.

The physiotherapists surveyed also indicated that only physiotherapists that have been officially trained and certified should be delivering Bobath treatment, a view which contradicts their previous opinion that all children with $\mathrm{CP}$ should receive Bobath treatment. 
This may not be feasible with the very limited numbers of therapists that are Bobath trained and are considered Bobath therapists.

Bobath therapists are usually senior therapists who have spent many years to earn their certifications that may extend to up to 10 weeks of training (Bobath Centre for Children with Cerebral Palsy). In many departments and institutes, senior therapists do not perform as many hands-on treatment hours as junior therapists, usually performing only consulting duties in the department rather than applied hands-on treatment. In cases similar to that, the expertise of these therapists is not put to maximal use in the departments which is not the ideal situation for children receiving physiotherapy treatment. Accordingly, not all children with $\mathrm{CP}$ will have the chance to receive physiotherapy treatment by such highly qualified therapists and waiting times will be further prolonged. Therefore, physiotherapists should be further educated to understand that Bobath may not be the best approach for children with CP neither is it the most effective and other physiotherapy interventions are also beneficial for children with CP. Understanding that other physiotherapy intervention is either as good as Bobath or show more significant results leads to a higher level of confidence when providing physiotherapy treatment and therefore better results.

In the part of the survey assessing if Bobath was delivered in the correct parameters the participants were divided between 'Yes', 'No' and 'I Don't Know'. The highest percentage showed that Bobath is not being delivered in correct parameters $(36.7 \%)$. Whereas in the parameters section of the treatment delivered the majority of therapists $(63.3 \%)$ have indicated delivering treatment for 1 hour twice a week. This indicates that even though a high percentage of the therapists are Bobath trained or under training they are not delivering the treatment sessions in the correct intensity. Some comments have referred to the lack of time and high load of patients indicating that higher intensities of treatment should be given even though not much evidence is available to support the need of more sessions than twice a week. Moreover, no action has been taken to improve the parameters to match the correct intensity required. One comment was found to indicate the need for a protocol and more standardized parameters of treatment for the application of Bobath for children with $\mathrm{CP}$ which is in contrast to the general principle of tailored treatment suited to the child's specific physical and mental disabilities or the family's needs. The involvement of the children and families in the physiotherapy treatment process is considered an important aspect in 
providing guidelines for physiotherapy treatment for each child rather than providing a fixed protocol for all children with CP.

As for the principles of the Bobath approach, the vast majority of the participants have agreed that it is effective in inhibiting tone, improving posture, increase muscle power, and inhibit primitive reflexes. Some therapists have justified their belief with reference to research evidence and some referred it to personal experience. However, a number of respondents are also therapists who are not Bobath certified which either indicates that they are using the same principles without referring them to the Bobath intervention, or that they believe in the effect of Bobath due to the high numbers of supporters it has even though it lacks the strong evidence to support it.

As for the high responses of the beneficial effect of the home treatment it indicates and confirms the importance of parents' commitment to support and maintain the changes and progress in the children's progress. More efforts are needed to educate and emphasize the importance of applying the home treatment continuously by the parents emphasizing the fact that no amount of treatment can be effective unless the progress made in treatment is carried over into their everyday life and activities (Velickovic \& Perat, 2005). Different methods of education must be recruited including lectures and practical training in addition to continuous support and monitoring of the families and caregivers.

However, the choices that the therapists have made was found to contradict the aims of the Bobath approach. Most of the therapists have selected the range of motion outcome measure even though the Bobath approach has limited effects on muscle length. This indicates a poor match between the intervention that the physiotherapists are providing and the choice of outcome measure used to assess the level of progress which may affect the level of goal achievement. In addition, the muscle shortening that children develop is a symptom due to the nature of the CP disability and is not a symptom specifically targeted by the Bobath approach. This indicates the need for further development of the physiotherapists' skills in selecting the most suitable outcome measure to assess the level of progress of their patients. 


\subsubsection{Home Treatment Services}

Despite knowing and believing that the home program had an effect upon the effectiveness of the Bobath approach, therapists did not make an effort to improve the level of commitment of the caregivers to the home program. However, it should be mentioned that regardless of the low adherence of the patients to the home program the therapists have still reported that changes have been detected in more than $70 \%$ of the cases of $\mathrm{CP}$. When suggesting the need to further educate the caregivers regarding the importance of the home program more than $88 \%$ of the therapists have agreed that the key to improved adherence was further education. Some therapists have suggested evaluating whether the caregiver is applying the home treatment correctly at each session other opinions had suggested extra training sessions for the caregivers to ensure the principles of inhibiting the unwanted reflexes in certain positions while still facilitating movement and balance in addition to gradually allowing the child to control his/her own movement by gradually reducing support (Mayston, 2005, Velickovic \& Perat, 2005).

Although these suggestions may improve the skills of the caregiver and therefore ensure the quality of the home treatment given, it does not necessarily improve the level of commitment and application of the home treatment. Other therapists have stressed the education option to improve the level of awareness and understanding of the need of the extra home effort that supports the clinical intervention and ensures continuity of the handling. Some therapists have suggested making the home program officially part of the treatment by stopping clinical treatment if it was found and confirmed that home treatment is not being applied, which may not be considered as an ethically accepted suggestion with respect to the patient's right of treatment and furthermore the patient should not have to suffer from the results of the lack of commitment from the caregivers. Other suggestions have indicated the need for support groups of other caregivers that may have a role in encouraging stronger commitment. 


\subsection{Conclusion}

A survey was created to assess the opinions and views of paediatric physiotherapists in Kuwait to conclude the overall application of physiotherapy for children with Cerebral Palsy in Kuwait. This study was intended to help us understand the views of the physiotherapists in order to justify the findings of improper practice identified in the clinical audit in chapter five. It was also intended to understand the views regarding the Bobath therapy in order to address incorrect beliefs and provide correct and standardised evidencebased practice for children with CP. About 64 paediatric physiotherapists were targeted with the electronic online survey using the "Survey Monkey" software and we obtained a response level of $47 \%$.

The survey results indicate that only a few therapists were found to be certified Bobath therapists or receiving certified training compared to the majority of the therapists that either has been supervised by Bobath therapists and are providing Bobath influenced treatment or have not been trained at all.

As for the outcome measurement tools, the therapists have chosen the ROM to be the most useable even though the GMFM had provided a more valid and reliable level of evidence in assessing the child' functional level as a whole. The therapists also have almost all agreed to believe that the principles of the Bobath treatment are effective in controlling the major outcomes of cerebral palsy even though a high percentage of them have not been officially trained.

In an effort to improve the effects of the Bobath approach the majority of therapists have agreed that promoting the application of home program should be encouraged and followed to achieve higher levels of adherence from the caregivers by inducing higher levels and variety of parents'/caregivers' education. 


\subsection{Limitations}

Limitations of this study had included:

- The difficulty of getting in contact with all departments of physiotherapy in Kuwait and access to all paediatric therapists.

- The limited number of internet-connected computers in the departments that were accessible for the therapists during working hours. In an effort to avoid this aspect manual surveys were made available for the therapists to fill on their own time which had then led to the normal survey limitations of prolonged response time or had increased the rate of non-respondents or even the loss of the manual survey papers.

- The level of understanding that might have led to different interpretation from one therapist to the other.

- The inclusion of incomplete surveys that did not include the answers to all the questions set.

- The lack of responses to the open questions and additional comments.

- The number of non-respondents even after allowing sufficient time for the return of the survey even when reminder text messages were distributed.

- The fact that the survey was distributed in English, which is the second language to all therapists in Kuwait including foreigners. The use of only one language has been found to be a barrier. 


\section{Chapter 9}

\section{General Discussion}

\subsection{Summary of Findings}

A gap in knowledge was identified in chapter three regarding the effects of home treatment in supporting the effects and outcomes of the Bobath approach. Although the Bobath principles stress the aspect of continuous treatment, the studies evaluating its effects failed to identify and promote methods of application that ensures continuity of its delivery in considerable costs. This from personal experience had limited effects seen on the children with $\mathrm{CP}$ in real practice. In many situations and due to many reasons, in Kuwait the children may not be able to receive continuous treatment which affects the outcomes of functional development. This causes the following course of Bobath to restore lost abilities rather than continue to achieve new set goals of developmental functions which may be a limitation hindering continuous effects of improvement that could be provided by Bobath.

According to that, Bobath had been proven in some studies explored in chapter three as being not highly persuasive in its effects compared to other available intervention it remains to be considerably popular in many countries as being the first choice of physiotherapy treatment for children with $\mathrm{CP}$. Therefore, it has been essential to promote supportive interventions that not only facilitate the continuity of physiotherapy management for the children with $\mathrm{CP}$ but also improve the outcomes of the Bobath provided in physiotherapy clinics in Kuwait. The audit had provided results that would help structure more effective home treatments in Kuwait that involve the parent's goals as well as suits the families style of daily living in order to avoid making the home treatment a burden that would lead to limited adherence from the parents.

This research had therefore aimed to evaluate the effects of involving parents in the management of children with cerebral palsy using the Bobath approach in Kuwait. The information gathered to fulfil the answers of this research had included searching the literature, service evaluation, piloting a clinical trial, in addition to including the qualitative views and opinions of parents and therapists summarised in the following discussion. 


\subsection{The Effects of the Main Physiotherapy Interventions in the Literature Identifying}

the Most Popular and most recent Physiotherapy Intervention Versus the Bobath Approach as the Most Common Type of Intervention in Paediatric Physiotherapy Management.

The Bobath approach seems to be less investigated in the last 10 years compared to other interventions of paediatric that became more popular in this duration such as mCIMT and Bimanual therapy. Even though studies of Bobath were inconclusive in the $80 \mathrm{~s}$ and $90 \mathrm{~s}$ and were difficult to effectively reproduce due to the limitations in the methodological and interventional descriptions efforts were not made to achieve stronger evidence to support the Bobath approach in the last ten years. This may be referred back to the rise of other interventions that showed good levels of outcomes in children with $\mathrm{CP}$ that were as good as Bobath or better.

The findings in the literature discussing the effects of the overall treatment available on children may serve as a source of information to advance clinical practice while they are not considered enough to provide data that is necessary to change practice and change treatment decisions. Studies have failed to provide the necessary data to change general treatment decisions for physiotherapy treatment of children with CP (Damiano, 2014). The majority of published studies have pointed out the main effects of group differences but with minor attention to individual difference given that cerebral palsy is quite a heterogeneous population varying widely in type, timing, location and extent of brain injury. Therefore, advance should be made in the evidence-based research for management of children with cerebral palsy (Damiano, 2014).

Studies assessing the effectiveness of Bobath had shown inconsistent evidence to support the effect on functional level improvement. Evidence found in favour of NDT or Bobath treatment in the category of slowing or preventing contractures and deformities in addition to improving quality of gait patterns and speed. Results were found to be of the highest efficiency when applying Bobath with other approaches that would help in supporting the child's needs as a whole and that would provide the child with a higher variety of techniques the would promote higher and more significant changes in functional levels in different environments. 
Findings indicate that Bobath could be recommended as an appropriate physiotherapy treatment approach for $\mathrm{CP}$; however, to ensure the continuation of the observed beneficial effects it is necessary for further investigations to be completed to evaluate persistence as another important principle associated with the application of Bobath. The results from this study combined with the description of the principles of Bobath postulates that a supporting intervention such as home program could be effective to ensure the maintenance or increasing the levels of outcomes generated during the physiotherapy led Bobath treatment sessions to ensure continual treatment at home and in different environments that involve parents and daily activities. As the Bobath approach was the main intervention that had influence management of children with CP in Kuwait it was essential to identify a supporting method that would improve its outcomes significantly in order to achieve maximal outcomes for children receiving physiotherapy in Kuwait. An investigation was performed as a systematic review in the following chapter.

\subsection{Identifying the Effects of Home Program in Supporting the Effectiveness of Clinical Physiotherapy Interventions Including Bobath Therapy Through a systematic review}

The systematic review revealed positive results seen for the home treatment in outcomes for children with $\mathrm{CP}$ and maintenance of these outcomes even after up to four weeks follow up. This was by encouraging parents to adhere to the home program by including the parents in the goals set for the home treatment by providing the parents with the choice of activities and exercises required. Exercises offered were functional and intended to promote the principles of Bobath including the inhibiting of unwanted reflexes in different position while still facilitating movement (Bobath, 1990, Quinton, 1997). This is done by promoting balanced movement and gradually reducing supportive methods (Bly \& Whiteside, 1997). Another important factor was the amount and complexity of the exercises. The key was to facilitate simple and manageable amounts of physiotherapy treatment and exercises in order to avoid stress put upon parents, which in turn would lead to low adherence.

Results also showed the education of families regarding the importance and the purpose of the home treatment played a significant part in promoting adherence at home, which was also supported by follow up through a variety of methods. This was to ensure 
availability of physiotherapists even when the child was not receiving clinical physiotherapy management.

An important factor that was found to significantly affect the cooperation of families was to be the level of stress that is applied on the families with a child with special needs especially with other siblings in the family. Therefore, the physiotherapists must also learn to evaluate and understand the level of stress and coping that families are facing in order to set achievable goals that do not further stress such families. According to that, different methods may be implemented in the home treatment to ensure full cooperation with the least efforts from both families and children.

Findings from this study were further implemented in Kuwait to be assessed in the pilot trial in chapter six. To ensure the cooperation of parents an educational course was provided and the home treatment application was monitored and following that in chapter seven the satisfaction of families was also assessed to evaluate the level of stress as part of it.

\subsection{Identifying Areas for Improvement in the Improper or Ineffective Delivered Services of Clinical Physiotherapy in the Physiotherapy Department in PMR Hospital in Kuwait to be Compared with the Results Learned from the Literature to be Reimplemented in Future Practice.}

In chapter five the audit was aimed to identify any areas that require improvement in the physiotherapy services provided for children with CP in Kuwait. limitations have been identified in the level of goal setting for the physiotherapy intervention provided. This led to the goals to be unmeasurable and therefore would be difficult to identify if these goals have been achieved at the end of the physiotherapy intervention and therefore results have identified the achievement of set goals to be less than $70 \%$. Other than that, the goals that have been set by the physiotherapists lack all manners of parents' involvement in creating the goals. In addition to that, the goals have shown no consideration of including daily activities in the home environment as one of the aspects in which the child's abilities must develop in. This is according to Butler et al., (2007) one of the principles of Bobath treatment in that the outcomes of therapy must be extended to include other environments such as the home environment in addition to the clinical environment. 
Furthermore, although all records assessed had shown documented home treatment exercises offered to patients, there was a lack in the follow up of the application of the home treatment and the support provided for the parents to perform the home treatment correctly and efficiently. According to Basaran et al (2014), the involvement of the parents/caregivers may have a high-level effect on both adherence to the program designed and the effect that could be seen in the children's progression given that the program was influenced by the everyday needs that the child faces in the daily activities at home. In addition, educating the parents regarding the home program did not appear to be mentioned. The methods in which the home program is taught may also play a role in the parents/caregivers understanding of the importance of its effects in addition to the role of efficient application of skills and parameters of the taught treatment.

This was also identified in the questionnaires in the pilot study in Kuwait of chapter six that have evaluated earlier received services. Parents have reported some aspects of dissatisfaction with the education provided throughout physiotherapy courses regarding their children's conditions and the education regarding the home treatment provided. Only 51\% were completely happy with the level of education provided in previous physiotherapy courses. In addition, about $80 \%$ of the physiotherapists in chapter 9 have agreed that more education of parents should be provided to promote better aspects of practice. Which in agreement to the literature in chapter three which identifies the importance of engaging the families in the overall goal setting and understanding of the nature of the children's disability to ensure that the physiotherapy intervention manages the child's problems throughout the day rather than focuses on specific movement problems in a few hours in the physiotherapy department (DeGangi \& Royeen, 1994; Knox \& Evans, 2002).

Therefore, in accordance to this, a pilot clinical trial had been created with improved levels of education to the parents, more objective assessment tool, and increased levels of follow up provided to the parents of children with CP for the application of home treatment. 


\subsection{Evaluating the Outcomes of Improved Aspects of Physiotherapy Management by Involving the Families of Children with $C P$ in a Pilot Clinical Trial that Includes Limitations Identified by the Clinical Audit to Improve the Outcomes of Physiotherapy Management in PMR Hospital in Kuwait.}

The outcome of the pilot study had shown some promising effects on the adherence to the home treatment in response to improved levels of education regarding the importance of applying the home treatment. These findings are beneficial compared to the compliance with the home treatment provided in the department with hardly any education regarding the importance and the impacts of its effects. Which should not be the case according to Novac and Cusick (2006) who have indicated in their study that family compliance may be useful in some approaches however they are not found very effective in supporting other approaches that focus on participation rather than just compliance. In another study (Wang et al., 2013) also indicated how low compliance is induced as a result of previously set physiotherapy treatment plans that do not include the parents in the overall understanding and goal set for the intervention.

This was highlighted in the results of the questionnaire that had evaluated the opinions of parents in Kuwait regarding previous home treatment deliveries, the participants have indicated in confirmation with the results of the clinical audit that they were not very committed to the frequency of the home program advised by the physiotherapists. As for the outcomes of home follow up, results had not shown favourable effects compared to education alone.

This may also promote that education and encouragement of parents to apply for the home program has the same if not better effects on adherence to the home treatment than the home visits provided and therefore it is considered a cost-effective method that promotes adherence to the treatment and does not apply additional burdens on the generals' costs of physiotherapy treatment. This also seemed to fulfil the two-major requirement of current health economic needs that include the ability to 1) Improve health outcomes through improved intervention strategies, and 2) The ability to convince the society and health care financiers to financially support such interventions (Saltman et al., 1997).

Perhaps other methods of follow up that are cost-effective such as the methods found in the literature (Chen et al., 2014; Katz-Leurer et al., 2009) that include a diary or a weekly 
phone call or even internet communication that may be implemented to facilitate encouragement and enquiry fulfilment of the parents.

The improvement of the level of adherence to the home treatment in this study had a positive effect on the maintenance and improvement of the functional level of the children with CP which was considered an important aspect in supporting Bobath influence management on the department of physiotherapy. Similarly, Basaran et al. (2014) had identified home treatment and the participation of the caregivers is crucial for the rehabilitation of disabled children. It was found to be effective in accelerating the success of the rehabilitation goals. Therefore, the adherence to the home program is one of the most important factors that maximise outcomes. On the other hand, failure to adhere to home treatment was proven to be one of the factors that affect treatment outcomes.

These results have been achieved as a result of a pilot experimental trial that had a limited population with limited financial support and had only demonstrated the potential of the supported Bobath intervention in improving functional outcomes of children with $\mathrm{CP}$ which might be further expanded to show higher levels of change in outcomes as a result of a randomised controlled trial.

\subsection{Evaluating Qualitative Satisfaction Responses of Parents Regarding the Integrity and Validity of the Pilot RCT in Kuwait.}

The satisfaction of parents with the services provided had been found according to Ygge and Arnetz (2001) to induce compliance and adherence to the medical regimens. Other findings were that in response to being satisfied and confident parents tend to reflect these reactions to their children and therefore promote higher levels of security. On the other hand, parents that experience anxiety regarding the interventions provided for their children reflects these feelings onto their children leading to anxious and fearful patients (Wharton et al, 1996). Moreover, the satisfaction of patients is considered an efficient and inexpensive method of assessing the quality of services (Edlund et al., 2003).

In this study, positive levels of satisfaction were seen as a result of the improved services of physiotherapy of the pilot study in Kuwait. These results might have been due to being involved in the physiotherapy treatment for their children and parents that participate in the treatment of their children would develop reduced levels of parental stress and therefore 
higher satisfaction levels. It might also have been a result of having an improved idea of the naturally expected prognosis of their children that promotes a higher level of acceptance of the functional developmental level of the children.

Communication, on the other hand, between patients and care providers promotes an important trust relationship and support to assist the parents in managing and developing through the stages of accepting and furthermore handling the cases that their children have. Communication also ensures a better understanding of nature and the effects of the intervention provided and what is expected from the intervention as well as the parents according to Piggot et al. (2002).

Satisfaction of the parents had also included the aspects of integrity between the information of the pilot study explained at the beginning of recruiting participants and the actual procedures that the families have experienced in the trial.

\subsection{Investigation of Physiotherapists Opinions of Clinical Physiotherapy Management} Featuring Bobath Therapy in Kuwait compared to the Best Practice that they Believe in addition to Identifying the Factors Affecting these Opinions.

Different opinions were found among physiotherapists in Kuwait regarding the effects of Bobath and the correct parameters of delivering the intervention for children with CP. Results had shown that even the therapists that had not been Bobath trained had thought Bobath is the best treatment for children with $\mathrm{CP}$.

However, the practice identified from the audit in chapter five does not indicate that the therapists are applying the principles of Bobath in inducing a continuous treatment approach that includes different environments, or the principle of involving the families further in understanding the nature of the disability, the nature of the intervention, and what is expected from them to support the intervention in different environments. This was in compliance with DeGangi and Royeen (1994) that had indicated that therapists do not necessarily apply in real practice the principles of the intervention that they have belief in.

When asked about the popularity of the Bobath approach about $80 \%$ of therapists in Kuwait had agreed that it is a widely-used approach around the world. Also, the majority of $53.3 \%$ had agreed that all children with $\mathrm{CP}$ should be receiving Bobath which are the percentage that represents the therapists that are trained or are providing Bobath influenced 
therapy even though more than half of the therapists did not agree that Bobath is considered the most effective treatment intervention compared to other interventions available which is in confirmation with Brown and Burns (2001).

It was also found that the $63.3 \%$ of therapists believed that only certified Bobath therapists or Bobath trained therapists should be providing Bobath therapy even though a high number of therapists $(43.3 \%)$ have not received any Bobath training. This question was suspected to be miss interpreted and that the therapists have believed that "trained therapists only should provide Bobath therapy" rather than "only Bobath trained therapists should provide Bobath therapy" which may have resulted in these percentages. If not, and if therapists believed that Bobath therapy must only be applied by Bobath therapists interprets to an obvious lack in their qualifications when managing the cases with $\mathrm{CP}$.

When investigating the opinions regarding the principles of Bobath even though some therapists were not Bobath qualified more than $63.3 \%$ have agreed on the effectiveness of all the Bobath principles for reducing symptoms and complications that might be hindering the functional development in children with CP. On the other hand, only $30 \%$ of the responses have agreed that Bobath is being delivered to the centres in the right parameters of treatment. Which is highly due to the wide range of variation of the parameters of Bobath that is being discussed in the literature (Fetters and Kluzik, 1996, Knox and Evans, 2002, Bar-Haim et al, 2006)

Changing the practice would require an effort to convince the therapists to replace the intervention that they prefer with the intervention $\mathrm{n}$ that is more effective as was also suggested by DeGangi \& Royeen (1994). In the efforts of improving the outcomes, $70 \%$ of the therapists agreed on the importance of the home program in supporting treatment which had justified their commitment to providing the home treatment to their patients. In the efforts of improving the level of adherence to the home program, the therapists have suggested group therapy, monthly educational lectures, in addition to one therapist that had suggested public campaigns. Other suggestions had included practising the home program in every session and discussing the difficulties that might be found during the application at home. None of the suggestions had indicated the inclusion of the parents in creating the home program to better serve the needs of the child and parent at home similar to what Sorsdahl et al. had suggested in 2010 that had shown improved results in parents' commitment to the home program application at home. Therefore, the results of this research must be considered 
in the development of a randomised controlled trial that would further explore these findings in the future.

\subsection{Recommendation for Research}

Further research must also consider the following recommendations to avoid complications that were found to affect the application of the studies in this research:

- Creating a policy of dealing with missing data in the patients' records by not including the data in the analysis or calculating results with respect to the total population that had complete records information. As for missing data in the questionnaire, a policy of reminders of the importance of completing the while questionnaire must be used, in addition to the exclusion of the missing data from the total analysed data

- The inclusion of a wider geographic area in order to include a larger population that would provide higher levels of evidence and generalisation of the results.

- Creating a team of physiotherapists that would be completely committed to the trial and not in order to provide assessments and treatment without being engaged in other work demands.

- Providing all participants, the chance to receive the experimental intervention following the end of the trial similar to a crossover method in order to encourage participation of patients and parents.

- Redesigning the questionnaire to include a more closed question to gather more data and avoid the use of many open-ended questions that might not be answered by the parents.

- Promote a higher level of discretion in the distribution and collection of the questionnaires in order to promote higher levels of integrity in the answers provided by the parents. 
- Providing a longer period of time for the response of the physiotherapist survey in order to ensure the collection of a larger number of responses.

- Providing the choice of paper copies of the survey for departments that do not provide internet services in their facilities.

- The ability to ask any questions regarding any part that was not understood in the survey through phone calls to ensure anonymity.

- Providing the survey in different languages.

\subsection{Recommendation for practice}

- Families will benefit if a weekly educational session was offered especially if there children were recently diagnosed with $\mathrm{CP}$ and were new to physiotherapy interventions. Important information regarding the disability and the prognosis of the condition will be of high value in that stage.

- Implementing group lectures may have the effect of support groups to the families.

- Private training sessions to focus on practicing hands on management and correcting insufficient handling will highly promote sufficient handling and management in the home setting.

- To promote adherence to home management a follow up method that is suitable for the families' choice such as a phone call, video call or a diary could have a significant effect.

- Overall parents/therapist cooperation may be improved by seeking feedback from families and promoting further communication with families.

\subsection{Impact on Practice}

The results of this research had developed a number of improvement aspects to be applied to physiotherapy for the management of children with CP. Some aspects have been addressed by promoting the principle that during the development of patient-specific 
treatment goals that they are informed by a standardized documentation process that directs physiotherapists to detail the precise assessment tool to detect changes in the child's condition, by linking this a scale that has an evidence base to it (such as the Goal Attainment Scale).

Furthermore, the literature suggests the involvement of parents in goal settings is an important aspect of reaching better adherence to the treatment and higher achievement of set goals and therefore better overall outcomes of management of children. Consideration must be given to involving the families of the children it is a concept that should be included in future approaches to create the goals that would provide overall improvement of functional abilities in the department and the home environment; therefore, providing an opportunity for a patient-centred approach to assist with collaboration between the physiotherapist and the parents/carers to support the continuous nature of provision of treatment, which is a fundamental principle of the Bobath approach.

Moreover, this research had identified some limitations in the standardization of the education or training provided to the parents regarding the nature of $\mathrm{CP}$, the prognosis of the condition in addition to the effects and importance of the application of home treatment (specifically in terms of compliance) to maximize any treatment effect of the Bobath therapy prescribed by the physiotherapist. In addition to that, the level of support provided to the parents to encourage the adherence to home treatment may be further developed and improved by the inclusion of a dedicated (and supported) protocol of support post physiotherapy treatment.

\subsection{Conclusion}

This research was planned to identify improved methods of delivering physiotherapy management via the Bobath approach. In order to achieve that the literature has been initially explored and critically appraised in chapter two and three in order to identify aspects that would help in the development of the parameters of improved practice. The literature had also assisted in supporting the design and methodologies of the studies used in this research to fulfil its aims. The clinical audit had assisted in chapter five in evaluating the services of physiotherapy applied in PMR hospital in Kuwait and therefore had assisted in identifying the limitations service delivery that might have had an effect on the levels of outcomes of 
functional development in children with $\mathrm{CP}$ and had limitations in the level of adherence to the home treatment by the parents. Therefore, a clinical trial was then performed to evaluate the effect of the developed services of parents' engagement in the continuous Bobath management of their children. This had resulted in improved levels of change especially observed in the periods of time that the children have not been receiving physiotherapy treatment in the department. The parents participating in this trial have shown high levels of satisfaction at the end of the trial. Results of the studies included were further compared to the opinions of the physiotherapists in Kuwait. This had identified results similar to those in the literature that may endure some levels of development. The pilot study of this research may further be escalated to an RCT due to the promising results observed where the sample size used may also be further developed to calculate the appropriate population size for a randomised controlled trial. Therefore, at the end of this research, the objectives of the studies have been fulfilled.

\subsection{Future Research}

Further research should be implemented to overcome the limitations faced in this research. A limited sample size was one of the most important limitations that must be avoided by including a larger sample size. Future study may also include a larger geographic area in Kuwait by including more than just one site of physiotherapy services. This will allow more variety of cases in addition to the chance of comparing different parameters and intensities of physiotherapy interventions and a higher level of generalisation of results to in Kuwait. Different methods of follow up for families may also be considered in a future study that may be less stressful and costing for the physiotherapy departments such as phone calls or video calls that will allow the physiotherapists to closely observe the treatment being delivered at home without the efforts of an actual visit. A reminder text may also be sent from the physiotherapist to the parents every other day to encourage home management. And automated text message may also be sent weekly to be ticked off by the families that includes the number of time that home treatment was provided each week. Electronic methods or methods of "social media" are considered easy to use and provide closer monitoring as well as removing boundaries between families and physiotherapists that allows high levels of communication and furthermore, stronger support. 


\subsection{Intention of Dissemination}

- Results of this research are intended to be published in medical and physiotherapy journals.

- Results of this research are intended to be taught to physiotherapy colleagues during workplace scientific lectures.

- Results of this research are intended to be taught to the families of children with CP through medical brochures or educational booklets. 


\section{References}

Aarts, P. B., Jongerius, P. H., Geerdink, Y. A., Van, L. J. \& Geurts, A. C. (2010) Effectiveness of modified constraint-induced movement therapy in children with unilateral spastic cerebral palsy: a randomized controlled trial. Neurorehabil Neural Repair, 24.

Adams, M.A., Chandler, L.S. and Schuhmann, K. (20000. Gait Changes in Children with Cerebral Palsy Following a Neurodevelopmental Treatment Course. Pediatric Physical Therapy, 12(3), pp.114-120.

Ahearne, C. E., Boylan, G. B., Murray, D. M. (2016). Short and long term prognosis in perinatal asphyxia: An update. World Journal of Clinical Pediatrics, 5(1), 67-74.

Ahl, L. E., Johansson, E., Granat, T. \& Carlberg, E. B. (2005) Functional therapy for children with cerebral palsy: an ecological approach. Developmental Medicine and Child Neurology, 47(9), 613-619.

Aicardi, J. (1992) Epileptic encephalopathies of early-childhood. Current Opinion in Neurology and Neurosurgery, 5(3), 344-348.

Ailon, T., Beauchamp, R., Miller, S., Mortenson, P., Kerr, J.M., Hengel, A.R. and Steinbok, P. (2015). Long-term outcome after selective dorsal rhizotomy in children with spastic cerebral palsy. Child's Nervous System, 31(3), pp.415-423.

Aisen. M., Kerkovich. D., Mast. J., Mulroy. S., Wren. T, Kay. R., Rethlefsen. S. (2011). Cerebral palsy: clinical care and neurological rehabilitation. The Lancet Neurology. 10(9): 844-852 
Akbari, A., ZADEH, M.J., Shahraki, S. and JAVARAN, P.J. (2009). The Effects of Functional Therapy on Motor Development in Children with Cerebral Palsy. Iranian Journal of Child Neurology, 3(3), pp.23-32.

Akbayrak, T., Armutlu, K., Gunel, M. K. \& Nurlu, G. (2005) Assessment of the shortterm effect of antispastic positioning on spasticity. Pediatrics International, 47(4), 440445 .

Anttila, H., Autti-Ramo, I., Suoranta, J., Makela, M. \& Malmivaara, A. (2008) Effectiveness of physical therapy interventions for children with cerebral palsy: A systematic review. Bmc Pediatrics, 8.

Armitage. P, Berry. G, Mathews. J. (2008). Statistical Methods in Medical Research. London. Wiley

Arnetz. B. (1999). Staff Perception of the Impact of Health Care Transformation on Quality of Care. International Journal of Quality of Health Care. 11: 345-351.

Barber, C. E. (2008) A guide to physiotherapy in cerebral palsy. Paediatrics and Child Health, 18(9), 410-413.

Bar-Haim, S., Harries, N., Belokopytov, M., Frank, A., Copeliovitch, L., Kaplanski, J. \& Lahat, E. (2006) Comparison of efficacy of Adeli suit and neurodevelopmental treatments in children with cerebral palsy. Developmental Medicine and Child Neurology, 48(5), 325-330.

Barrett, J. A., Evans, L., Chappell, J., Fraser, C., \& Clayton, L. (2001). Bobath or Motor Relearning Programme. Clinical rehabilitation, 15(4), 445-446. 
Basaran. A, Karadavut. K, Uneri. S, Balbaloglu.O, Atasoy. N. (2014). Adherence to home exercise program among caregivers of children with cerebral palsy. Turkish Journal of Physical Medicine \& Rehabilitation. 60: 85-91

Benjamin. A. (2008). Audit: How to do in Practice. BMJ. 336: 1214-1245

Bickman. L. (2000). Are You Satisfied with Satisfaction? Mental Health Services Research, 2, p. 125.

Bilde, P.E., Kliim-Due, M., Rasmussen, B., Petersen, L.Z., Petersen, T.H. and Nielsen, J.B., 2011. Individualized, home-based interactive training of cerebral palsy children delivered through the Internet. BMC neurology, 11(1), p.32.

Blair, C. and Wahlsten, D.,(2002). Why early intervention works: A reply to Baumeister and Bacharach. Intelligence, 30(2), pp.129-140.

Blundell, S.W., Shepherd, R.B., Dean, C.M., Adams, R.D. and Cahill, B.M. (2003). Functional strength training in cerebral palsy: a pilot study of a group circuit training class for children aged 4-8 years. Clinical Rehabilitation, 17(1), pp.48-57.

Bobath. K. (1992). A neurophysiological basis for the treatment of cerebral palsy. $3^{\text {rd }}$ Edition. Cambridge University Press.

Bower. E, Michell. D, Burnett. M, Campbell. M, Mclellan. D. (2001). Randomized controlled trial of physiotherapy in 56 children with cerebral palsy followed for 18 months. Developmental Medicine and Child Neurology. 43: 4-15. 
Bowling. A. (2014). Research Method in Health: Investigating Health and Health Services. $4^{\text {th }}$ edition. Berkshire. Open University Education McGraw Hill Education.

Boyer, K. K., Olson, J. R., Calantone, R. J., \& Jackson, E. C. (2002). Print versus electronic surveys: a comparison of two data collection methodologies. Journal of Operations Management, 20(4), 357-373.

Brandao, M. B., Ferre, C., Kuo, H. C., Rameckers, E. A. A., Bleyenheuft, Y., Hung, Y. C., Friel, K. \& Gordon, A. M. (2014) Comparison of Structured Skill and Unstructured Practice During Intensive Bimanual Training in Children With Unilateral Spastic Cerebral Palsy. Neurorehabilitation and Neural Repair, 28(5), 452-461.

Brophy. S, Snooks. H, Grifiths. L. (2008). Small Scale Evaluation in health: A Practical Guide. London. Sage Publication Ltd.

Bruce. N, pope. D, Stanistreet. D. (2008). Quantitative Mrthods for Health Research: A Practical interactive Guide to Epidemiology and Statistics. NJ. John Wiley \& sons.

Brunton, L.K. and Bartlett, D.J., (2011). Validity and reliability of two abbreviated versions of the Gross Motor Function Measure. Physical therapy, 91(4), p.577.

Bryanton, C., Bosse, J., Brien, M., Mclean, J., McCormick, A. and Sveistrup, H., (2006). Feasibility, motivation, and selective motor control: virtual reality compared to 
conventional home exercise in children with cerebral palsy. Cyberpsychology \& behavior, 9(2), pp.123-128.

Burtner, P. A., Leinwand, R., Sullivan, K. J., Goh, H., \& Kantak, S. S. (2014). Motor learning in children v hemiplegic cerebral palsy: Feedback effects on skill acquisition. Developmental Medicine and C Neurology, 56(3), 259-266.

Butler, C., \& Darrah. J. (2001). Effects of neurodevelopmental treatment (NDT) for cerebral palsy: An AACPDM evidence report. Developmental Medicine and Child Neurology, 43(11), 778-790.

Butler, C., Darrah, J., Adams, R., Chambers, H., Abel, M., Damiano, D., Edgar, T., Msall, M., Samson-Fang, L., Stott, N. S., Law, M., Leach, J., Goldstein, M., O'Donnell, M. \& McLaughlin, J. (2007) Effects of neurodevelopmental treatment (NDT) for cerebral palsy: An AACPDM evidence report. Developmental Medicine and Child Neurology, 43(11), 778-790.

$\begin{array}{lllll}\text { Causes of } & \text { CP. } & \text { [Illustration]. 2016. Retrieved }\end{array}$ http://neonatology.ucsf.edu/specialized- care/cerebral-palsy.aspx.

Chambers, R., \& Wakley, G. (2005). Clinical audit in primary care: demonstrating quality and outcomes. Radcliffe Publishing.

Campbell, S.K., Palisano, R.J. and Vander Linden, D.W.,( 2006). Physical therapy for children. Saunders. 
Charles, J. \& Gordon, A. M. (2006) Development of hand-arm bimanual intensive training (HABIT) for improving bimanual coordination in children with hemiplegic cerebral palsy. Developmental Medicine and Child Neurology, 48(11), 931-936.

Chatburn. R. (2011). Hand Book for Healthcare Research. $2^{\text {nd }}$ edition. London. Jones and Bartlett Publishers

Chen, K., Ren, Y., Gaebler-Spira, D. and Zhang, L.Q. (2014) Home-based tele-assisted robotic rehabilitation of joint impairments in children with cerebral palsy. In Engineering in Medicine and Biology Society (EMBC), 2014 36th Annual International Conference of the IEEE (pp. 5288-5291). IEEE.

Cheyne, T. L., \& Ritter, F. E. (2001). Targeting audiences on the internet.

Communications of the ACM, 44(4), 94-98.

Chiarello. L, Palisano. R, Maggs. J, Orlin. M, Almasri. N, Kang. L, Chang. H. (20100. Family priorities for activity and participation of children and youth with cerebral palsy. Physical Therapy. 90,9: 1254-1264.

Chochowska, M., Zgorzalewicz-Stachowiak, M. and Sereda-Wiszowaty, E., (2008). Wpływ wybranych czynników na skuteczność metody NDT-Bobath w usprawnianiu dzieci z mózgowym porażeniem dziecięcym. Fizjoterapia, 16(3), pp.8-24.

Coker, P., Lebkicher, C., Harris, L. and Snape, J., (2009). The effects of constraintinduced movement therapy for a child less than one year of age. NeuroRehabilitation, 24(3), pp.199-208. 
Creswell, J. W., Klassen, A. C., Plano Clark, V. L., \& Smith, K. C. (2011). Best practices for mixed methods research in the health sciences. Bethesda (Maryland): National Institutes of Health, 2094-2103.

Curtis, E., \& Drennan, J. (2013). Quantitative Health Research: Issues And Methods: Issues and Methods. McGraw-Hill Education (UK).

Curtis, D.J., Bencke, J. and Mygind, B., (2014). The effect of training in an interactive dynamic stander on ankle dorsiflexion and gross motor function in children with cerebral palsy. Developmental neurorehabilitation, 17(6), pp.393-397.

Dalvand, H., Dehghan, L., Feizy, A., Amirsalai, S. \& Bagheri, H. (2009) Effect of the Bobath Technique, Conductive Education and Education to Parents in Activities of Daily Living in Children with Cerebral Palsy in Iran. Hong Kong Journal of Occupational Therapy, 19(1), 14-19.

Damiano, D.L., Dodd, K. and Taylor, N.F., 2002. Should we be testing and training muscle strength in cerebral palsy?. Developmental Medicine \& Child Neurology, 44(01), pp.68-72.

Damiano, D. L. (2009) Rehabilitative Therapies in Cerebral Palsy: The Good, the Not As Good, and the Possible. Journal of Child Neurology, 24(9), 1200-1204.

Damiano, D. L. (2014) Meaningfulness of mean group results for determining the optimal motor rehabilitation program for an individual child with cerebral palsy. Developmental Medicine and Child Neurology, 56(12), 1141-1146. 
Darrah, J., Watkins, B., Chen, L. and Bonin, C., (2004). Conductive education intervention for children with cerebral palsy: an AACPDM evidence report. Developmental medicine \& child neurology, 46(3), pp.187-203.

Darrah, J., Law, M. C., Pollock, N., Wilson, B., Russell, D. J., Walter, S. D., Rosenbaum, P. \& Galupp, B. (2011) Context therapy: a new intervention approach for children with cerebral palsy. Developmental Medicine and Child Neurology, 53(7), 615-620.

DeGangi, G.A. and Royeen, C.B., (1994). Current practice among neurodevelopmental treatment association members. American Journal of Occupational Therapy, 48(9), pp.803-809.

DeLuca, S. C., Echols, K., Law, C. R. \& Ramey, S. L. (2006) Intensive pediatric constraint-induced therapy for children with cerebral palsy: randomized, controlled, crossover trial. J Child Neurol, 21.

Demuth, S.K., Knutson, L.M. and Fowler, E.G., (2012). The PEDALS stationary cycling intervention and health-related quality of life in children with cerebral palsy: a randomized controlled trial. Developmental Medicine \& Child Neurology, 54(7), pp.654- 661.

Desloovere, K., De Cat, J., Molenaers, G., Franki, I., Himpens, E., Van Waelvelde, H., Fagard, K. \& Van den Broeck, C. (2012) The effect of different physiotherapy interventions in post-BTX-A treatment of children with cerebral palsy. European Journal of Paediatric Neurology, 16(1), 20-28. 
Dodd, K.J., Taylor, N.F. and Graham, H.K.,(2004). Strength training can have unexpected effects on the self-concept of children with cerebral palsy. Pediatric Physical Therapy, 16(2), pp.99-105.

Dong, V. A., Tung, I. H., Siu, H. W. \& Fong, K. N. (2013) Studies comparing the efficacy of constraint-induced movement therapy and bimanual training in children with unilateral cerebral palsy: a systematic review. Dev Neurorehabil, 16.

Drouin, L.M., Malouin, F., Richards, C.L. and Marcoux, S., (1996). Correction between the gross motor function measure scores and gait spatiotemporal measures in children with neurological impairments. Developmental Medicine \& Child Neurology, 38(11), pp.1007-1019.

Edlund. M,. Young. A, Kung. F, Sherbourne. C, Wells. C. (2003). Does satisfaction reflect the technical quality of mental health care? Health Services Research, 38 pp. 631-645.

Eliasson, A. C., Shaw, K., Berg, E. \& Krumlinde-Sundholm, L. (2011) An ecological approach of constraint induced movement therapy for 2-3-year-old children: a randomized control trial. Res Dev Disabil, 32.

Embrey, D.G., Yates, L. and Mott, D.H., (1990). Effects of neuro-developmental treatment and orthoses on knee flexion during gait: a single-subject design. Physical Therapy, 70(10), pp.626-637.

Eriksson. C, Katz-salamon. M, Carlberg. E. (2006). Early motor assessment in very preterm born infants as predictor of performance at 5.5 years. Advances in Physiotherapy. 8: $175-181$ 
Fetters, L. \& Kluzik, J. A. (1996) The effects of neurodevelopmental treatment versus practice on the reaching of children with spastic cerebral palsy. Physical Therapy, 76(4), 346-358.

Fitzpatrick. S. 2006. Clinical Trial Designs. Buckinghamshire. The Institute of Clinical Research.

Franki, I., Desloovere, K., De Cat, J., Feys, H., Molenaers, G., Calders, P., ... \& Van den Broeck, C. (2012). The evidence-base for basic physical therapy techniques targeting lower limb function in children with cerebral palsy: a systematic review using the International Classification of Functioning, Disability and Health as a conceptual framework. Journal of Rehabilitation Medicine, 44(5), 385-395.

Franki, I., Van den Broeck, C., De Cat, J., Tijhuis, W., Molenaers, G., Vanderstraeten, G., \& Desloovere, K. (2014). A randomized, single-blind cross-over design evaluating the effectiveness of an individually defined, targeted physical therapy approach in treatment of children with cerebral palsy. Clinical rehabilitation, 28(10), 1039-1052.

Garland. A, Saltzman. M, Aarons. G. (2000). Adolescents' Satisfaction with Mental Health Services. Development of a Multi-Dimensional Scale. Evaluation and Program Planning, 23 pp. $165-175$

Garland. A, Haine. R, Boxmeyer. C. (2007). Determinates of Youth and Parent Satisfaction in Usual Care Psychotherapy. Evaluation and Program Planning. 30 (1) pp: $45-54$ 
Golomb, M.R., Warden, S.J., Fess, E., Rabin, B., Yonkman, J., Shirley, B. and Burdea, G.C., (2011). Maintained hand function and forearm bone health 14 months after an inhome virtual-reality videogame hand telerehabilitation intervention in an adolescent with hemiplegic cerebral palsy. Journal of child neurology, 26(3), pp.389-393.

Gordon, A.M., Schneider, J.A., Chinnan, A. and Charles, J.R., (2007). Efficacy of a hand-arm bimanual intensive therapy (HABIT) in children with hemiplegic cerebral palsy: a randomized control trial. Developmental Medicine \& Child Neurology, 49(11), pp.830-838.

Gottwald, M., \& Lansdown, G. (2014). Clinical Governance: Improving The Quality Of Healthcare For Patients And Service Users. McGraw-Hill Education (UK).

Graham, J. V., Eustace, C., Brock, K., Swain, E., \& Irwin-Carruthers, S. (2009). The Bobath concept in contemporary clinical practice. Topics in stroke rehabilitation, 16(1), 57-68.

Grimshaw, J. M., Shirran, L., Thomas, R., Mowatt, G., Fraser, C., Bero, L., O'Brien, M. A. (2001). Changing provider behavior: an overview of systematic reviews of interventions. Medical care, II2-II45.

Hall, M. (2009). Early recognition of developmental delays. The Journal for Nurse Practitioners, 5(9), 690-691.

Halvarsson, S., Asplund, R. and Fjellman-Wiklund, A., (2010). From authority to coachParents' experiences of stretching as a home programme for children with cerebral palsy. Advances in Physiotherapy, 12(4), pp.208-216. 
Harbourne. R, Willet. S, Kyvelidou. A, Deffeyes. J, Stergiou. N. (2010). A comparison of interventions for children with cerebral palsy to improve sitting postural control: A clinical trial. Physical Therapy. 90, 12: 1881-1898.

Harris, S. R., \& Roxborough, L. (2005). Efficacy and effectiveness of physical therapy in enhancing postural control in children with cerebral palsy. Neural plasticity, 12(2-3), 229243.

Hertzog, M. A. (2008). Considerations in determining sample size for pilot studies. Research in nursing \& health, 31(2), 180-191.

Hickson. M. (2013). Research handbook for Health Care Professionals. London. John Wiley and sons.

Himmelmann, K., Beckung, E., Hagberg, G. \& Uvebrant, P. (2007) Bilateral spastic cerebral palsy-prevalence through four decades, motor function and growth. European Journal of Paediatric Neurology, 11(4), 215-222.

Himpens, E., Oostra, A., Franki, I., Vansteelandt, S., Vanhaesebrouck, P. and Van den Broeck, C.,(2010). Predictability of cerebral palsy in a high-risk NICU population. Early human development, 86(7), pp.413-417.

Hurn, J., Kneebone, I., \& Cropley, M. (2006). Goal setting as an outcome measure: a systematic review. Clinical rehabilitation, 20(9), 756-772.

International Classification of Function, Disability and Health (ICF). 2001, World Health Organization, Geneva.

Intrathecal Baclofen Pump. [Illustration] (2016) Retrieved from http://www.rch.org.au/kidsinfo/fact sheets/Intrathecal baclofen pictures/ 
Jameson, R., Rech, C. and De Loubresse, C.G., (2010). Cervical myelopathy in athetoid and dystonic cerebral palsy: retrospective study and literature review. European Spine Journal, 19(5), pp.706-712.

Josenby. A, Jarnlo. G, Gummesson. C, Nordmark. E. (2009). Longitudinal Construct Validity of the GMFM-88 Total Score and Goal Total Score and the GMFM-66 Score in a 5-Year Follow Up Study. Physical Therapy. 89 (4): 342-350

Katz-Leurer. M, Rotem. H, Keren. O, Meyer. S. 2009. The effects of a home-based taskoriented exercise programme on motor and balance performance in children with spastic cerebral palsy and severe traumatic brain injury. Clinical Rehabilitation. 23: 714-724

Ketelaar, M., Kruijsen, A. J. A., Verschuren, O., Jongmans, M. J., Gorter, J. W., Verheijden, J., Reinders-Messelink, H. A. \& Lindeman, E. (2010) LEARN 2 MOVE 2-3: a randomized controlled trial on the efficacy of child-focused intervention and contextfocused intervention in preschool children with cerebral palsy. Bmc Pediatrics, 10, 10.

Kielhofner.G. 2006. Research in Occupational Therapy: Methods for Enquiry for Enhancing Practice. Philadelphia: F. A. Davis.

Kiresuk, T. J., Smith, A., \& Cardillo, J. E. (2014). Goal attainment scaling: Applications, theory, and measurement. Psychology Press.

Klingels, K., Feys, H., Molenaers, G., Verbeke, G., Van Daele, S., Hoskens, J., Desloovere, K. \& De Cock, P. (2013) Randomized Trial of Modified Constraint-Induced 
Movement Therapy With and Without an Intensive Therapy Program in Children With Unilateral Cerebral Palsy. Neurorehabilitation and Neural Repair, 27(9), 799-807.

Kluzik, J., Fetters, L., Coryell, J. \& Scholz, J. P. (1990) Quantification of control: A preliminary study of effects of neurodevelopmental treatment on reaching in children with spastic cerebral palsy. Physical Therapy, 70(2), 65-78.

Knox, V. \& Evans, A. L. (2002) Evaluation of the functional effects of a course of Bobath therapy in children with cerebral palsy: A preliminary study. Developmental Medicine and Child Neurology, 44(7), 447-460.

Ko, J., \& Kim, M. (2013). Reliability and responsiveness of the gross motor function measure-88 in children with cerebral palsy. Physical therapy, 93(3), 393.

Krigger, K.W., (2006). Cerebral palsy: an overview. Am Fam Physician, 73(1), pp.91-100

Krägeloh-Mann, I., (2008). Understanding causation of cerebral palsy by using magnetic resonance imaging. paediaTRics and cHild HealTH, 18(9), pp.399-404.

Kuban, K., Allred, E. N., O'Shea, T. M., Paneth, N., Pagano, M., Dammann, O., Du Plessis, A., Westra, S., Miller, C., Bassan, H., Krishnamoorthy, K., Junewick, J., Olomu, N., Romano, E., Seibert, J., Engelke, S., Karna, P., Batton, D., O'Connor, S., 
Keller, C. \& Leviton, A. (2008) Cranial ultrasound lesions in the NICU predict cerebral palsy at age 2 years in children who were born at extremely low gestational age. Annals of Neurology, 64, S81-S81.

Lagunju. I and Adedokun. B. (2008). A comparison of quadriplegic and hemiplegic cerebral palsy. Journal of Pediatric Neurology. 6: 25-30.

Larsson, I., Miller, M., Liljedahl, K. \& Gard, G. (2012) Physiotherapists' experiences of physiotherapy interventions in scientific physiotherapy publications focusing on interventions for children with cerebral palsy: a qualitative phenomenographic approach. Bmc Pediatrics, 12, 12.

Law, M., Russell, D., Pollock, N., Rosenbaum, P., Walter, S. \& King, G. (1997) A comparison of intensive neurodevelopmental therapy plus casting and a regular occupational therapy program for children with cerebral palsy. Dev Med Child Neurol, 39.

Law, M. C., Darrah, J., Pollock, N., Wilson, B., Russell, D. J., Walter, S. D., Rosenbaum, P. \& Galuppi, B. (2011) Focus on function: a cluster, randomized controlled trial comparing child- versus context-focused intervention for young children with cerebral palsy. Developmental Medicine and Child Neurology, 53(7), 621-629.

Levitt. S. (2004). Treatment of Cerebral Palsy and Motor Delay. 4th Edition. Wiley - Blackwell.

Lilly, L. A. \& Powell, N. J. (1990) Measuring the effects of neurodevelopmental treatment on the daily living skills of 2 children with cerebral palsy. The American 
journal of occupational therapy. : official publication of the American Occupational Therapy Association, 44(2), 139-145.

Lukban, M.B., Rosales, R.L. and Dressler, D., (2009). Effectiveness of botulinum toxin A for upper and lower limb spasticity in children with cerebral palsy: a summary of evidence. Journal of neural transmission, 116(3), pp.319-331.

MacLennan, A., (1999). A template for defining a causal relation between acute intrapartum events and cerebral palsy: international consensus statement. BMJ: British Medical Journal, 319(7216), p.1054.

MacLennan. A., Thompson. S., Gecz. J. (2015). Cerebral palsy: causes, pathways, and the role of genetic variants. American Journal of Obstetrics \& gynecology. 213(6):779-88

Mahoney. G, Robinson. C, Perales. F. (2004). Early motor intervention, the need for new treatment paradigms. Infants and Young Children. 17, 4: 291-300.

Mannion, A. F., Caporaso, F., Pulkovski, N., \& Sprott, H. (2010). Goal attainment scaling as a measure of treatment success after physiotherapy for chronic low back pain. Rheumatology, keq160.

Marino.B, Marino. E. (2000). Report of Children Hospital Care: What it Means for your Practice. Pediatric Nursing. 26 (2) pp: 195-198.

Marret, S., Vanhulle, C.A.T.H.E.R.I.N.E. and Laquerriere, A.N.N.I.E., (2013). Pathophysiology of cerebral palsy. Handb Clin Neurol, 111, pp.169-176. 
Mayston, M., (2005). Evidence-based physical therapy for the management of children with cerebral palsy. Developmental Medicine \& Child Neurology, 47(12), pp.795-795.

Mayston am, M., (2011). From ‘one size fits all’to tailor-made physical intervention for cerebral palsy. Developmental Medicine \& Child Neurology, 53(11), pp.969970.

McCarthy, M. L., Silberstein, C. E., Atkins, E. A., Harryman, S. E., Sponseller, P. D., \& Hadley-Miller, N. A. (2002). Comparing reliability and validity of pediatric instruments for measuring health and well-being of children with spastic cerebral palsy.

Developmental Medicine \& Child Neurology, 44(7), 468-476.

McSherry. R, Pearce. P. (2011). Clinical Governance: A Guide to Implementation for Healthcare Professionals. $3^{\text {rd }}$ edition. West Sussex. John Wiley and sons Ltd.

Miller.F. (2005). Cerebral Palsy. New York. Springer Science + Business media.

Moumtzoglou. A, Dafogianni. C, Karra. V, Michailidou. D, Panagiota. L, Bartsocas. C. (2000) Development and Application of a Questionnaire for Assessing Parent Satisfaction with Care. International Journal for Quality in Health Care. 12 (4) pp: 331-337.

Moye. L. (2013). Statistical Reasoning in Medicine: The Intuitive P-Value Primer. New York. Springer Verlag.

Myrhaug, H. T., Ostensjo, S., Larun, L., Odgaard-Jensen, J. \& Jahnsen, R. (2014) Intensive training of motor function and functional skills among young children with cerebral palsy: a systematic review and meta-analysis. Bmc Pediatrics, 14, 19. 
National Health Services. 2009. NPSA Annual Report \& Accounts. Retrieved from: www.npsa.nhs.uk/EasySiteWeb/GatewayLink.aspx?alld=74433.

National Institute for Health and Care Excellence.

2002. https://www.nice.org.uk/guidance?unlid.

Nelson, L., Owens, H., Hynan, L. S., Iannaccone, S. T., \& Group, A. (2006). The gross motor function measure ${ }^{\mathrm{TM}}$ is a valid and sensitive outcome measure for spinal muscular atrophy. Neuromuscular Disorders, 16(6), 374-380.

Newell. R, Burnard. P. (2011). Research for Evidence Based Practice in Healthcare. $2^{\text {nd }}$ edition. West Sussex. Blackwell Publishing.

NHS Clinical Governance Support Team. 2005. A practical handbook for clinical audit.www.cgsupport.nhs.uk/Resources/Clinical_Audit/7@,Appendix_1.asp.

Nordmark, E., Jarnlo, G.B. and Hägglund, G., (2000). Comparison of the Gross Motor Function Measure and Paediatric Evaluation of Disability Inventory in assessing motor function in children undergoing selective dorsal rhizotomy. Developmental Medicine \& Child Neurology, 42(4), pp.245-252.

Norman. G, Streiner. D. (2003). PDQ Statistics. $3^{\text {rd }}$ edition. Shelton. People's Medical Publishing House.

Novak, I. and Cusick, A., (2006). Home programmes in paediatric occupational therapy for children with cerebral palsy: Where to start? Australian Occupational Therapy Journal, 53(4), pp.251-264. 
Novak, I., Cusick, A. and Lowe, K., (2007). A pilot study on the impact of occupational therapy home programming for young children with cerebral palsy. The American journal of occupational therapy, 61(4), p.463.

Novak, I., McIntyre, S., Morgan, C., Campbell, L., Dark, L., Morton, N., Stumbles, E., Wilson, S. A. \& Goldsmith, S. (2013) A systematic review of interventions for children with cerebral palsy: state of the evidence. Dev Med Child Neurol, 55.

Orthotic Braces. [Illustration] (2016) Retrieved from:

http://www.getdomainvids.com/keyword/orthotic\%20brac

es/.

Palisano. R, Hanna. S, Rosenbaum. P, Russel. D, Walter. S, Wood. E, Raina. P, Galuppi.

B. (2000). Validation of a model of gross motor function for children with cerebral palsy. Physical Therapy. 80: 974-985

Palmer, F.B., (2004). Strategies for the early diagnosis of cerebral palsy. The Journal of pediatrics, 145(2), pp.S8-S11.

Panteliadis. C. (2011). Cerebral Palsy. Stuttgart: Verlag.

Papavasiliou, A. S. (2009) Management of motor problems in cerebral palsy: A critical update for the clinician. European Journal of Paediatric Neurology, 13(5), 387-396.

Pape. K. (2012). Developmental and maladaptive plasticity in neonatal SCI. Clinical Neurology and Neurosurgery. 114 (5): 475-482. 
Piggot, J., Hocking, C. and Paterson, J., (2003). Parental adjustment to having a child with cerebral palsy and participation in home therapy programs. Physical \& Occupational Therapy in Pediatrics, 23(4), pp.5-29.

Piggot, J., Paterson, J. and Hocking, C., (2002). Participation in home therapy programs for children with cerebral palsy: A compelling challenge. Qualitative Health Research, 12(8), pp.1112-1129.

Plishta. S, Garzon. L. (2009). Statistics for Nursing and Allied Health. New York. Lippincott Williams and Wilkins.

Polgar. S, Thomas. S. (2013). Research in the Health Sciences. $6^{\text {th }}$ edition. Sydney. Churchill Livingstone Elsevier.

Pope, C., Mays, N., Ziebland, S., le May, A., Williams, S., Coombs, M., ... \& Ellis, J. (2000). Qualitative methods in health research. methods, 1, 2.

Portney. L, Watkins. M. (2015). Foundation of Clinical Research-application to practice. $4^{\text {th }}$ edition. New Jersey: Prentice-Hall Health.

Potentially Fetal Hemorrhage. [Illustration]. (2016). Retrieved from http://www.stanfordchildrens.org/en/service/neonatology/prenatal-steroidsreduce- preemies-risk-for-brain-bleeding.

Raine. S. (2007). The current theoretical assumption of the Bobath concept as determined by the members of BBTA. Physiotherapy Theory and Practice, 23(3):137-152.

Reddihough. D, Collins. K. (2003). The epidemiology and causes of cerebral palsy. Australian Journal of Physiotherapy. 49: 7-12. 
Reid. L., Rose. S., Boyd. R. (2015). Rehabilitation and neuroplasticity in children with unilateral cerebral palsy. Nature Reviews Neurology. Advance Online Publication.

Ridding. M., MKay. D., Thompson. P., Miles. T. (2001). Changes in corticomotor representations induced by prolonged peripheral nerve stimulation in humans. Clinical Neurophysiology, 112 (8):1461-9.

Rosenbaum. P, Palisano. R, Barlett. D, Galuppi. B, Russel. D. (2008). Development of the gross motor function classification system for cerebral palsy. Developmental Medicine and Child Neurology. 50: 249-253.

Russel. D, Avery. L, Rosenbaum. P, Raina. P, Walter. S, Palisano. R. (2000). Improved scaling of the gross motor function measure for children with cerebral palsy: evidence of reliability and validity. Physical Therapy. 80: 873-885.

Russell. D, Rosenbaum. P, Avery. L, Lane. M. (2002). Gross Motor Function Measure (GMFM-66 and GMFM-88): User’s Manual. London. MacKeith Press.

Saks. M, Allsop. J. (2013). Researching Health: qualitative, Quantitative, and Mixed Methods. $2^{\text {nd }}$ edition. London. Sage Publications Ltd.

Sakzewski, L., Ziviani, J. \& Boyd, R. N. (2014) Efficacy of upper limb therapies for unilateral cerebral palsy: a meta-analysis. Pediatrics, 133. 
Salem, Y., \& Godwin, E. M. (2009). Effects of task-oriented training on mobility function in children with cerebral palsy. NeuroRehabilitation, 24(4), 307-313.

Saltman, R.B., Figueras, J. and Saltman, R.B., (1997). European health care reform: analysis of current strategies (pp. 203-245). Copenhagen: World Health Organization, Regional Office for Europe.

Sankar, C. \& Mundkur, N. (2005) Cerebral palsy-definition, classification, etiology and early diagnosis. Indian Journal of Pediatrics, 72(10), 865-868.

Scheinberg, A., Hall, K., Lam, L.T. and O'Flaherty, S., (2006). Oral baclofen in children with cerebral palsy: A double-blind cross-over pilot study. Journal of paediatrics and child health, 42(11), pp.715-720.

Scherzer, Alfred L. (2000). Early Diagnosis and Interventional Therapy in Cerebral Palsy: An Interdisciplinary Age-Focused Approach. $3^{\text {rd }}$ edition. Marcel Dekker. NewYork.

Scianni, A., Butler, J.M., Ada, L. and Teixeira-Salmela, L.F., (2009). Muscle strengthening is not effective in children and adolescents with cerebral palsy: a systematic review.

Australian Journal of Physiotherapy, 55(2), pp.81-87.

Shamsoddini, A. (2010) Comparison between the effect of neurodevelopmental treatment and sensory integration therapy on gross motor function in children with cerebral palsy. Iranian Journal of Child Neurology, 4(1), 31-38.

Siegert, R. J., \& Levack, W. M. (Eds.). (2014). Rehabilitation Goal Setting:

Theory, Practice and Evidence. CRC Press. 
Sim. J, Wright. C. (2000). Research in Health Care. Cheltenham. Stanley Thornes Ltd.

Smania, N., Aglioti, S. M., Cosentino, A., Camin, M., Gandolfi, M., Tinazzi, M., Fiaschi, A. \& Faccioli, S. (2009) A modified constraint-induced movement therapy (CIT) program improves paretic arm use and function in children with cerebral palsy. European Journal of Physical and Rehabilitation Medicine, 45(4), 493-500.

Smith. S, Senclair. D, Raine. R. (2005). Health Care Evaluation. London. Open University Education McGraw Hill Education.

Sorsdahl, A. B., Moe-Nilssen, R., Kaale, H. K., Rieber, J. \& Strand, L. I. (2010) Change in basic motor abilities, quality of movement and everyday activities following intensive, goal-directed, activity-focused physiotherapy in a group setting for children with cerebral palsy. BMC Pediatrics, 10.

Steele, K.M., van der Krogt, M.M., Schwartz, M.H. and Delp, S.L., (2012). How much muscle strength is required to walk in a crouch gait?. Journal of biomechanics, 45(15), pp.2564-2569.

Steenbeek, D., Gorter, J. W., Ketelaar, M., Galama, K., \& Lindeman, E. (2011). Responsiveness of Goal Attainment Scaling in comparison to two standardized measures in outcome evaluation of children with cerebral palsy. Clinical rehabilitation, 25(12), 1128-1139.

Tang. M, Lin. C, Lin. W, Chen. C, Tsai. S, Chang. Y. (2011). The effect of adding a home program to weekly institutional-based therapy for children with undefined developmental delay: a pilot randomised clinical trial. Journal of the Chinese Medical Association. 74: 259-266 
Taylor, N.F., Dodd, K.J., McBurney, H. and Graham, H.K., (2004). Factors influencing adherence to a home-based strength-training programme for young people with cerebral palsy. Physiotherapy, 90(2), pp.57-63.

Taylor. G. (2005). Integrating Quantitative and qualitative Methods in Research. $2^{\text {nd }}$ edition. Mary Land. University of Press of America.

Taylor. C, Groot. J, Blair. E, Stanley. F. (2009). The risk of cerebral palsy in survivors of multiple pregnancies with cofetal loss or death. American Journal of Obstetrics and Gynecology. 201, 41: 1-6

The Pyramid of evidence hierarchy. [Illustration] (2017) Retrieved from: http://www.students4bestevidence.net/the-evidence-based-medicinepyramid.

Thompson, C. B., \& Panacek, E. A. (2007). Research study designs: Non-experimental. Air medical journal, 26(1), 18-22.

Trahan, J., \& Malouin, F. (1999). Changes in the Gross Motor Function Measure in Children with Different Types of Cerebral Palsy: An Eight-Month Follow-Up Study. Pediatric Physical Therapy, 11(1), 12-17.

Tsorlakis, N., Evaggelinou, C., Grouios, G. \& Tsorbatzoudis, C. (2004) Effect of intensive neurodevelopmental treatment in gross motor function of children with cerebral palsy. Developmental Medicine and Child Neurology, 46(11), 740-745. 
Twycross, A., \& Shorten, A. (2014). Service evaluation, audit and research: what is the difference? Evidence Based Nursing, ebnurs-2014.

Types of Cerebral Palsy[Illustration](2016) Retrieved from: : www.slideshare.net .

Vanu Som, C. (2004). Clinical governance: a fresh look at its definition.

Clinical Governance: An International Journal, 9(2), 87-90.

Velickovic. T., Perat. M. (2005). Basic Principles of Neurodevelopmental Treatment. Medicina, 42 (41):112-120

Wang, T.H., Peng, Y.C., Chen, Y.L., Lu, T.W., Liao, H.F., Tang, P.F. and Shieh, J.Y., (2013). A Home-Based Program Using Patterned Sensory Enhancement Improves Resistance Exercise Effects for Children with Cerebral Palsy a Randomized Controlled Trial. Neurorehabilitation and neural repair, 27(8), pp.684694.

Weindling, A. M., Cunningham, C. C., Glenn, S. M., Edwards, R. T. \& Reeves, D. J. (2007) Additional therapy for young children with spastic cerebral palsy: a randomised controlled trial. Health Technol Assess, 11.

Weisz. J, Doss. A, Hawley. K. (2005). Youth Psychotherapy Outcome Research: A Review and Critique of the evidence Base. Annual Review of Psychology. 56, pp: 337363.

Wharton. R, Levine. K, Buka. S, Emanuel. L. (1996). Advance Care Planning for Children with Special Health Care Needs: A Survey of parental Attitudes. Pediatrics. 97: 682-687. 
Whittingham, K., Wee, D. and Boyd, R. (2011). Systematic review of the efficacy of parenting interventions for children with cerebral palsy. Child: care, health and development, 37(4), pp.475-483.

Wood. E, Rosenbaum. P. (2000). The gross motor function classification system for cerebral palsy: A study of reliability and stability overtime. Developmental Medicine and Child Neurology. 42: 292-296.

Wood, E. (2006) The child with cerebral palsy: diagnosis and beyond.

Seminars in pediatric neurology, 13(4), 286-96.

Ygge. B, Arnetz. J. (2001). Quality of Pediatric Care: Application and Validation of an Instrument for Measuring Parent Satisfaction with Hospital Care. International Journal for Quality of Health Care. 13 (1): pp. 33-43. 


\section{APPENDIX 1}

GROSS MOTOR FUNCTION MEASURE (GMFM) SCORE SHEET (GMFM-88 and GMFM-66 scoring)

Child's Name:

Assessment Date:
ID\#:

\section{GMFCS Level $^{1}$}




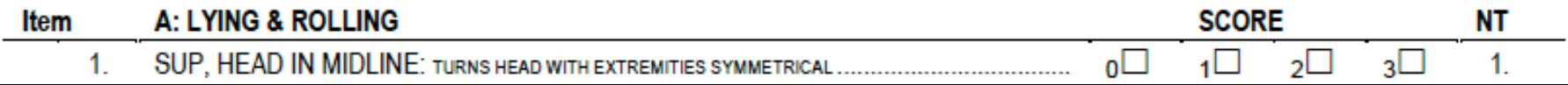

$\begin{array}{llll}1 & 2\end{array}$




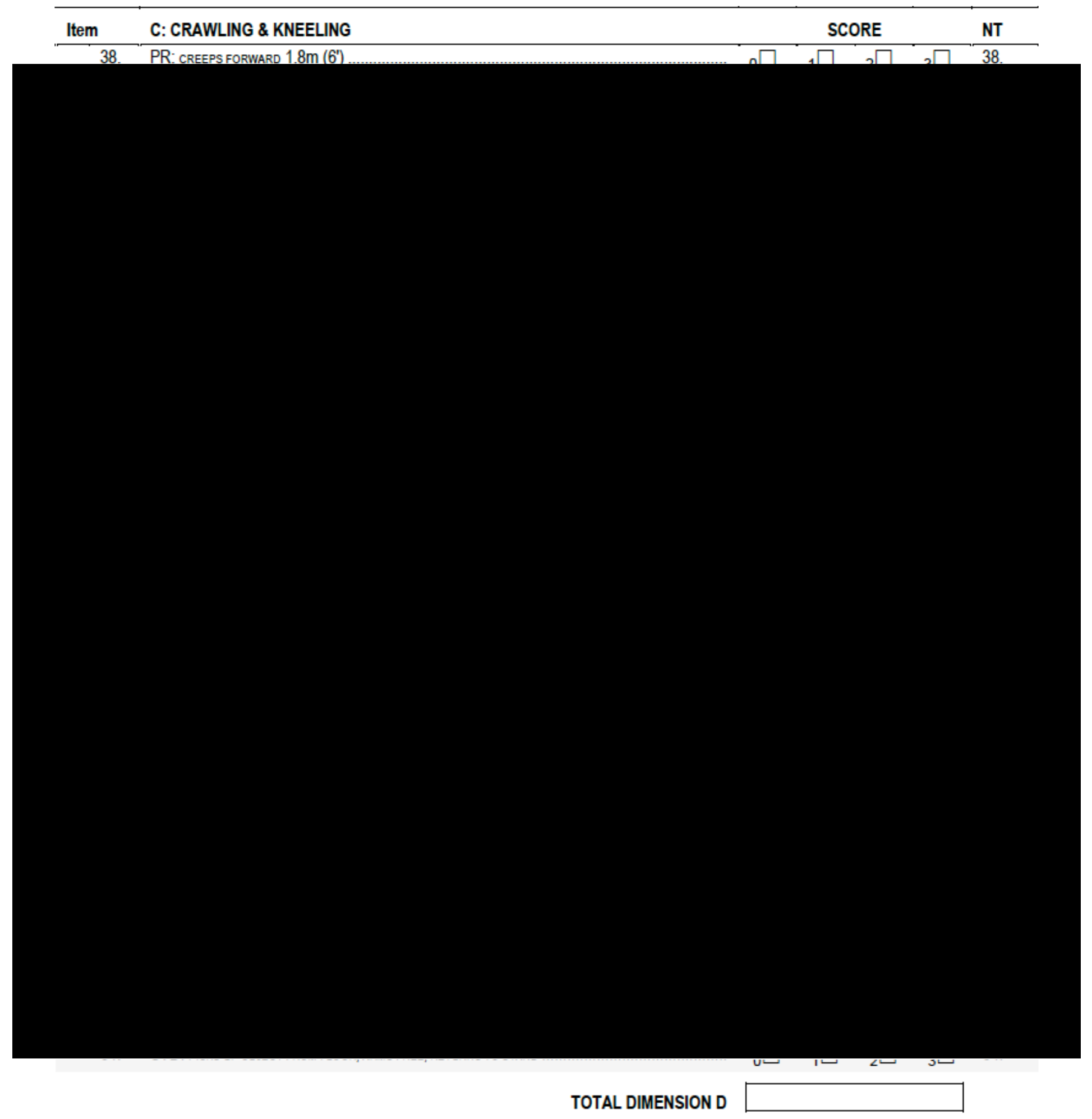


* 65. STD, 2 HANDS ON LARGE BENCH: CRUIIES 5 STEPS TO $R$

${ }_{1} \square \quad 2 \square$

65.

STD 2 HAMRS ON IRCE RENCH.

$0 \square$

$\square \quad \square$


GMFM-88 SUMMARY SCORE

GOAL

AREA 


\section{TESTING WITH AIDS/ORTHOSES USING THE GMFM-88}

Indicate below with a check (4) which aid/orthosis was used and what dimension it was first applied. (There may be more than one)

AID

Rollator/pusher

Walker
Dimension

$\square$
Orthosis

Hip Control

Knee Control
Dimension

$\square$ 


\section{APPENDIX 2}

\section{Application of the GMFM}

\section{- How is the GMFM administered?}

- The GMFM should be administered in an environment that is comfortable for the child and is large enough to hold the necessary equipment and allow the child to move freely.

- The floor must be a smooth and firm surface.

- As the GMFM is used to measure change over time the environment and assessment conditions must be maintained as consistent as possible.

- The GMFM requires the child to demonstrate various motor skills as outlined in the GMFM scoring sheet. While an observing examiner evaluates and scores the skill.

- The GMFM must be used by pediatric therapists who are familiar with assessing motor skills in children.

- The GMFM may take up to 45 to 60 minutes for someone familiar with the measure depending on the skill of the assessor and the ability level of the child and the level of cooperation and understanding.

- Depending on the child's age and level of understanding, the child is asked to perform the skill with detailed explanation of the required skill, or is encouraged with a toy to perform the skill.

- The child is given up to 3 chances to perform the skill if not executed correctly in the first trial.

- After the third trial, the skill is scored on the scoring sheet by the observing examiner.

- After the evaluation is completed the scores of each skill is calculated to produce a final total GMFM score. 


\title{
Appendix 3
}

\section{CASP}

\section{0 questions to help you make sense of qualitative research}

\author{
How to use this appraisal tool
}

Three broad issues need to be considered when appraising the report of a qualitative research:

- Are the results of the review valid?

- What are the results?

- Will the results help locally?

The 10 questions on the following pages are designed to help you think about these issues systematically. The first two questions are screening questions and can be answered quickly. If the answer to both is "yes", it is worth proceeding with the remaining questions.

There is some degree of overlap between the questions, you are asked to record a "yes", "no" or "can't tell" to most of the questions. A number of italicised prompts are given after each question. These are designed to remind you why the question is important. Record your reasons for your answers in the spaces provided.

These checklists were designed to be used as educational tools as part of a workshop setting There will not be time in the small groups to answer them all in detail! 


\section{Screening Questions}

1. Was there a clear statement of the aims

$\boldsymbol{r}_{\text {Yes }} \boldsymbol{r}_{\text {Can't tell }} \boldsymbol{r}_{\text {No }}$ of the research?

HINT: Consider

- What was the goal of the research?

- Why it was thought important?

- Its relevance

2. Is a qualitative methodology appropriate?

$\boldsymbol{r}_{\text {Yes }} \boldsymbol{r}_{\text {Can't tell }} \boldsymbol{r}_{\text {No }}$

HINT: Consider

- If the research seeks to interpret or illuminate the actions and/or subjective experiences of research participants

- Is qualitative research the right methodology for addressing the research goal? 
Is it worth continuing? 


\section{Detailed questions}

3. Was the research design appropriate to address the aims of the research?

HINT: Consider

- If the researcher has justified the research design (e.g. have they discussed how they decided which method to use)?

4. Was the recruitment strategy appropriate to the aims of the research?

HINT:Consider

- If the researcher has explained how the participants were selected

- If they explained why the participants they selected were the most appropriate to provide access to the type of knowledge sought by the study

- If there are any discussions around recruitment (e.g. why some people chose not to take part) 


\section{Was the data collected in a way that addressed}

\section{$\Gamma_{\text {Yes }} \boldsymbol{r}$ Can't tell $\boldsymbol{r}_{\text {No }}$}

\section{the research issue?}

\section{HINT: Consider}

- If the setting for data collection was justified

- If it is clear how data were collected (e.g. focus group, semi-structured interview etc.)

- If the researcher has justified the methods chosen

- If the researcher has made the methods explicit (e.g. for interview method, is there an indication of how interviews were conducted, or did they use a topic guide)?

- If methods were modified during the study. If so, has the researcher explained how and why?

- If the form of data is clear (e.g. tape recordings, video material, notes etc)

- If the researcher has discussed saturation of data

\section{participants been adequately considered?}

\section{HINT: Consider}

- If the researcher critically examined their own role, potential bias and influence during

(a) Formulation of the research questions

(b) Data collection, including sample recruitment and choice of location

- How the researcher responded to events during the study and whether they considered the implications of any changes in the research design 


\section{Have ethical issues been taken into consideration?}

HINT: Consider

- If there are sufficient details of how the research was explained to participants for the reader to assess whether ethical standards were maintained

- If the researcher has discussed issues raised by the study (e.g. issues around informed consent or confidentiality or how they have handled the effects of the study on the participants during and after the study)

- If approval has been sought from the ethics committee

\section{Was the data analysis sufficiently rigorous?}

HINT: Consider

- If there is an in-depth description of the analysis process

- If thematic analysis is used. If so, is it clear how the categories/themes were derived from the data? Whether the researcher explains how the data presented

- were selected from the original sample to demonstrate the analysis process

If sufficient data are presented to support the findings To what extent contradictory data are taken into account

- Whether the researcher critically examined their own role,

- potential bias and influence during analysis and selection

- of data for presentation 
HINT: Consider

- If the findings are explicit

- If there is adequate discussion of the evidence both for and against the researchers arguments If the researcher has discussed the credibility of their

- findings (e.g. triangulation, respondent validation, more than one analyst)

If the findings are discussed in relation to the original

- research question

\section{How valuable is the research?}

HINT: Consider

- If the researcher discusses the contribution the study makes to existing knowledge or understanding e.g. do they consider the findings in relation to current practice or policy?, or relevant research-based literature? If they identify new areas where research is necessary If the researchers have discussed whether or how the findings can be transferred to other populations or considered other ways the research may be used 


\title{
11 questions to help you make sense of case control study
}

\author{
How to use this appraisal tool
}

Three broad issues need to be considered when appraising a case control study:

- Are the results of the trial valid?

(Section A)

- What are the results?

(Section B)

- Will the results help locally?

(Section C)

The 11 questions on the following pages are designed to help you think about these issues systematically.

The first two questions are screening questions and can be answered quickly. If the answer to both is "yes", it is worth proceeding with the remaining questions.

There is some degree of overlap between the questions, you are asked to record a "yes", "no" or "can't tell" to most of the questions. A number of italicised prompts are given after each question. These are designed to remind you why the question is important. Record your reasons for your answers in the spaces provided.

These checklists were designed to be used as educational tools as part of a workshop setting There will not be time in the small groups to answer them all in detail!

(C) CASP This work is licensed under the Creative Commons Attribution - NonCommercial-ShareAlike 3.0 Unported License. To view a copy of this license, visit http://creativecommons.org/licenses/by-nc-sa/3.0/ www.casp-uk.net 


\section{(A) Are the results of the study valid?}

Screening Questions

1. Did the study address a clearly focused issue?

CYes

Can't tell $\boldsymbol{r}_{\text {No }}$

HINT: A question can be focused in terms of

- The population studied

- The risk factors studied

- Whether the study tried to detect a beneficial

or harmful effect?

2. Did the authors use an appropriate method to answer their question?

\section{HINT: Consider}

- Is a case control study an appropriate way of

Answering the question under the circumstances?

(Is the outcome rare or harmful)

- Did it address the study question?

\section{Is it worth continuing?}




\section{Were the cases recruited in an acceptable}

way?

HINT: We are looking for selection bias which might compromise validity of the findings

- Are the cases defined precisely?

- Were the cases representative of a defined population? (geographically and/or temporally?)

- Was there an established reliable system for selecting all the cases

- Are they incident or prevalent?

- Is there something special about the cases?

- Is the time frame of the study relevant to disease/exposure?

- Was there a sufficient number of cases selected?

- Was there a power calculation?

\section{Were the controls selected in an} acceptable way?

HINT: We are looking for selection bias which might compromise The generalisibilty of the findings

- Were the controls representative of defined population (geographically and/or temporally)

-Was there something special about the controls?

- Was the non-response high? Could non-respondents be different in any way?

- Are they matched, population based or randomly selected?

-Was there a sufficient number of controls selected? 

minimise bias?

HINT: We are looking for measurement, recall or classification bias

- Was the exposure clearly defined and accurately measured?

- Did the authors use subjective or objective measurements?

- Do the measures truly reflect what they are supposed to measure? (Have they been validated?)

- Were the measurement methods similar in the cases and controls?

- Did the study incorporate blinding where feasible?

- Is the temporal relation correct? (Does the exposure of interest precede the outcome?) authors accounted for?

HINT: List the ones you think might be important, that The author missed.

- Genetic

- Environmental

- Socio-economic

(b) Have the authors taken account

of the potential confounding factors in the design and/or in their analysis?

HINT: Look for

- Restriction in design, and techniques e.g. modelling stratified-, regression-, or sensitivity analysis to correct, control or adjust for confounding factors 


\section{What are the results of this study?}

HINT: Consider

- What are the bottom line results?

- Is the analysis appropriate to the design?

- How strong is the association between exposure and outcome (look at the odds ratio)?

- Are the results adjusted for confounding, and might confounding still explain the association?

- Has adjustment made a big difference to the OR?

\section{(B) What are the results?}

\section{How precise are the results?}

How precise is the estimate of risk?

HINT: Consider

- Size of the P-value

- Size of the confidence intervals

- Have the authors considered all the important variables?

- How was the effect of subjects refusing to participate evaluated?

9. Do you believe the results?

HINT: Consider

- Big effect is hard to ignore!

- Can it be due to chance, bias or confounding?

- Are the design and methods of this study sufficiently flawed to make the results unreliable? Consider Bradford Hills criteria (e.g. time sequence,

- dose-response gradient, strength, biological plausibility) 


\section{(C) Will the results help locally?}

10. Can the results be applied to the local

\section{population?}

HINT: Consider whether

- The subjects covered in the study could be sufficiently different from your population to cause concern

Your local setting is likely to differ much

- from that of the study

Can you quantify the local benefits and harms?

11. Do the results of this study fit with other available evidence?

HINT: Consider all the available evidence from RCT's, systematic reviews, cohort studies and case-control studies as well for consistency.

\section{Remember}

One observational study rarely provides sufficiently robust evidence to recommend changes to clinical practice or within health policy decision making.

However, for certain questions observational studies provide the only evidence.

Recommendations from observational studies are always stronger when supported by other evidence. 


\section{1 questions to help you make sense of a trial}

How to use this appraisal tool

Three broad issues need to be considered when appraising the report of a randomised controlled trial:

- Are the results of the trial valid?

- What are the results?

- Will the results help locally?
(Section A)

(Section B)

(Section C)

The 11 questions on the following pages are designed to help you think about these issues systematically.

The first two questions are screening questions and can be answered quickly. If the answer to both is yes, it is worth proceeding with the remaining questions.

There is some degree of overlap between the questions, you are asked to record a yes, no or can't tell to most of the questions. A number of prompts are given after each question. These are designed to remind you why the question is important. Record your reasons for your answers in the spaces provided.

There will not be time in the small groups to answer them all in detail!

These checklists were designed to be used as educational tools as part of a workshop

(C)ASP This work is licensed under the Creative Commons Attribution - NonCommercial-ShareAlike 3.0 Unported License. To view a copy of this license, visit http://creativecommons.org/licenses/by-nc-sa/3.0/. www.casp-uk.net 


\section{(A) Are the results of the trial valid?}

\section{Screening Questions}

1. Did the trial address a clearly focused issue?

$\boldsymbol{r}_{\text {Yes }} \boldsymbol{r}_{\text {Can't tell }} \boldsymbol{r}_{\text {No }}$

Consider: An issue can be 'focused' In terms of

- The population studied

- The intervention given

- The comparator given

- The outcomes considered

2. Was the assignment of patients to treatments randomised?

Consider:

- How was this carried out, some methods

may produce broken allocation concealment

-Was the allocation concealed from researchers?

\section{Is it worth continuing?}


Detailed questions

3. Were patients, health workers and study

res $r$ Can't tell $\boldsymbol{r}_{\mathrm{N}}$ personnel blinded?

Consider:

- Health workers could be; clinicians, nurses etc

- Study personnel - especially outcome assessors

4. Were the groups similar at the start of the trial?

$\boldsymbol{r}_{\text {Yes }} \boldsymbol{r}_{\text {Can't tell }} \boldsymbol{r}_{\text {No }}$

Consider: Look at

- Other factors that might affect the outcome such as age,

sex, social class, these may be called baseline characteristics

5. Aside from the experimental intervention, were the groups treated equally? 
6. Were all of the patients who entered the trial properly accounted for at its conclusion?

\section{Consider:}

- Was the trial stopped early?

- Were patients analysed in the groups to which they were randomised?

\section{(B) What are the results?}

7. How large was the treatment effect?

Consider:

-What outcomes were measured?

- Is the primary outcome clearly specified?

-What results were found for each outcome?

- Is there evidence of selective reporting of outcomes?
8. How precise was the estimate of the treatment effect?

Consider:

- What are the confidence limits?

- Were they statistically significant? 


\section{(C) Will the results help locally?}

9. Can the results be applied in your context?

ryes $\boldsymbol{r}_{\text {Can't tell }} \boldsymbol{r}$ No

(or to the local population?)

Consider:

- Do you have reason to believe that your population

of interest is different to that in the trial

- If so, in what way?

10. Were all clinically important outcomes

ryes $\boldsymbol{r}$ Can't tell $\boldsymbol{r}_{\mathrm{No}}$ considered?

Consider:

- Is there other information you would like to have seen?

- Was the need for this trial clearly described?

11. Are the benefits worth the harms and costs?

res $\Gamma$ Can't tell $\Gamma$ No

Consider:

- Even if this is not addressed by the trial, what do you think? 


\title{
10 questions to help you make sense of a review
}

\author{
How to use this appraisal tool
}

Three broad issues need to be considered when appraising the report of a systematic review:

- $\quad$ Are the results of the review valid?

- What are the results?

- Will the results help locally?
(Section A)

(Section B)

(Section C)

The 10 questions on the following pages are designed to help you think about these issues systematically.

The first two questions are screening questions and can be answered quickly. If the answer to both is "yes", it is worth proceeding with the remaining questions.

There is some degree of overlap between the questions, you are asked to record a "yes", "no" or "can't tell" to most of the questions. A number of prompts are given after each question. These are designed to remind you why the question is important. Record your reasons for your answers in the spaces provided.

These checklists were designed to be used as educational tools as part of a workshop setting There will not be time in the small groups to answer them all in detail! 


\section{(A) Are the results of the review valid?}

Screening Questions

1. Did the review address a clearly focused question?

$\boldsymbol{r}_{\text {Yes }} \boldsymbol{r}_{\text {Can't tell }} \boldsymbol{r}_{\text {No }}$

HINT: An issue can be 'focused' In terms of

- The population studied

- The intervention given

- The outcome considered

2. Did the authors look for the right type of papers?

ryes $\boldsymbol{r}$ Can't tell $\boldsymbol{r}$ No

HINT: 'The best sort of studies' would

- Address the reviews question

- Have an appropriate study design (usually RCTs for papers evaluating interventions)

\section{Is it worth continuing?}


3. Do you think all the important, relevant studies were included?

HINT: Look for

- Which bibliographic databases were used

- Follow up from reference lists

- Personal contact with experts

- Search for unpublished as well as published studies

- Search for non-English language studies

4. Did the review's authors do enough to assess the quality of the included studies?

HINT: The authors need to consider the rigour of the studies they have identified. Lack of rigour may affect the studies' results. ("All that glisters is not gold" Merchant of Venice - Act II Scene 7)

5. If the results of the review have been combined, was it reasonable to do so?

HINT: Consider whether

- The results were similar from study to study

- The results of all the included studies are clearly displayed

- The results of the different studies are similar

- The reasons for any variations in results are discussed 


\section{(B) What are the results?}

6. What are the overall results of the review?

HINT: Consider

- If you are clear about the review's 'bottom line' results

- What these are (numerically if appropriate)

- How were the results expressed (NNT, odds ratio etc)

7. How precise are the results?

HINT: Look at the confidence intervals, if given 


\section{(C) Will the results help locally?}

8. Can the results be applied to the local population?

$\boldsymbol{r}_{\text {Yes }} \boldsymbol{\Gamma}_{\text {Can't tell }} \boldsymbol{r}_{\text {No }}$

HINT: Consider whether

- $\quad$ The patients covered by the review could be sufficiently different to your population to cause concern

- Your local setting is likely to differ much from that of the review

9. Were all important outcomes considered?

ryes $\boldsymbol{r}$ Can't tell $\boldsymbol{r}_{\mathrm{N}}$

HINT: Consider whether

- Is there other information you would like to have seen

10. Are the benefits worth the harms and costs?

HINT: Consider

- Even if this is not addressed by the review, what do you think?

$\boldsymbol{r}_{\text {Yes }} \boldsymbol{r}_{\text {Can't tell }} \boldsymbol{r}_{\mathrm{N}}$ 


\section{APPENDIX 4}

\section{Kuwait Ethics}

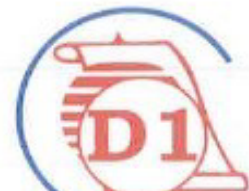

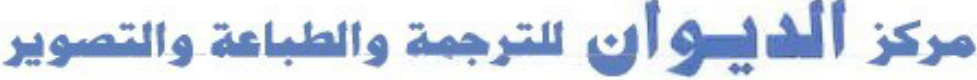 \\ Al-Diwan Translation Center E-mail: diwan_translation@hotmail.com}

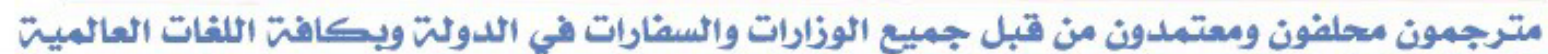
SWORN TRANSLATORS SPECIALIZED IN ALL APPROVED LANGUAGES AUTHORIZED BY ALL EMBASSIES \& MINISTRIES State of Kuwait Reference: wmtj/1527/2013

Ministry of Health Date: $18 / 08 / 2013$

Asst. Undersecretary for Planning \& Quality

Dear Sir; Dr. Undersecretary

Subject: facilitation the mission of the researcher Mrs./ Mona

Suleiman Al Matter and Dr. Suad Al-Enezi, Head of the Al-

Fahd Dep. of physical therapy hospital, research No. 25/2013

Under title;

\section{Evaluating Parental Interventions to Support the Management}

\section{of Children Cerebral Palsy}

Kindly be informed that the constituted standing committee by the virtue of the ministerial decision No. 207 of 2012, which concerned with the process of co-coordinating health and medical research has discussed, in its fifth meeting held on Monday, dated 25/06/2013, research protocol submitted by: Mona suleiman 


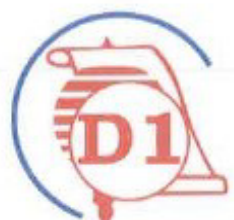

\section{مركز اللهبـأب أن الترجمة والطباعة والتصوير A1-Diwan Translation Center \\ E-mail: diwan_translation@hotmail.com}

مترجمون محلفون ومعتملدون من قبل جميع الوزارات والسفارات في الدولتي وبكافت اللغات العالميتي SWORN TRANSLATORS SPECIALIZED IN ALL APPROVED LANGUAGES AUTHORIZED BY ALL EMRASSIES \& MINISTRIES Almatr and physician Soaad Al-Enzi, head of Al-Fahd Dep. Of physical therapy hospital, thesis came under the title:

\section{Evaluating Parental Interventions to Support the Management of Children Cerebral Palsy}

Following the procedure took by the said committee of reviewing the opinions of the relevant parts concerning with the subject matter of the research pursuant to the circular issued by $\mathrm{Mr}$. Undersecretary under No. 156 of 2012 in this respect, where it received the approval of the following Messrs.:

Director of Administration of Physical Therapy Services by the virtue of the letter No. wmtj/577/2013, dated 08/05/2013.

Dr. Chief of the Board of Pediatrics Departments, dated $14 / 05 / 2013$.

- Dr. Chief of the Board Internal Medicine Departments under letter No. wmtj/617/2013. 


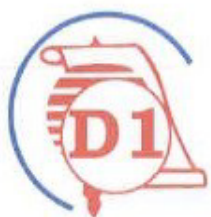

\section{مركز الليـئ أن الترجمة والطباعة والتصوير \\ Al-Diwan Translation Center \\ E-mail: diwan_translation@hotmail.com}

مترجمون محلفون ومعتملدون من قبل جميع الوزارات والسفارات في الدولتّ وبكافت اللفات العالهيت SWORN TRANSLATORS SPECIALIZED IN ALL APPROVED LANGUAGES AUTHORIZED BY ALL EMBASSIES \& MINISTRIES Corresponding to $15 / 05 / 2013$ and the request filed by $\mathrm{Mr}$.

Undersecretary of Legal and Investigations Affairs by the virtue of letter No. us/21/1054, dated 30/05/2013, for making some amendments related to research protocol and informed consent, the two researchers have made the necessary amendments, accordingly they submitted it to the Administration of Legal and Investigations Affairs, where the latter has given its consent under letter No. wmtj/1380/2013, dated 17/07/2013, therefore it approved and recommended to accept the research's thesis (under letter No. us/21/1474, dated 05/08/2013).

Therefore, on the said recommendations of the approval of the committee to conduct the research, the said researches have made the required amendments on the protocol and informed consent in accordance with the governing laws thereof.

Kindly review and advise accordingly, in respect with appealing th relevant parties who have something to do with the subject raatter

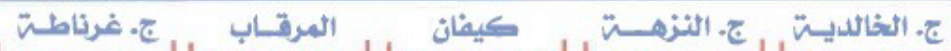
24864644 22462020 24816060 22531818 24926388 
مترجهون محلفون ومعتمدون من قبل جميع الوزارات والسفارات في الدولتي وبكافت اللفات العالميتً SWORN TRANSLATORS SPECIALIZED IN ALL APPROVED LANGUAGES AUTHORIZED BY ALL EMBAASIIES \& MINISTRIES

of the thesis (Mr. Director of Sabah Specialized Medical

Department: Director of Physical Therapy Hospital and Mr. Director

of Physical Therapy Services) to take all the necessary actions to

facilitate the mission of the two researches in their research.

The said researchers shall observe all the rights of privacy and

confidentiality of patients and not disclosing any information outside

the scope of the thesis of whatsoever.

Best regards

Asst. Undersecretary for Planning \& Quality

Dr. Gamal Mansour Alharbi

Asst. Undersecretary of Ministry of Health

For Technical Affairs

(Sealed \& signed)

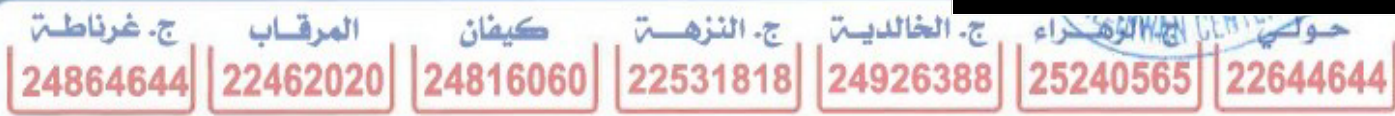




\section{Reference :}

Date :

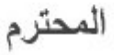

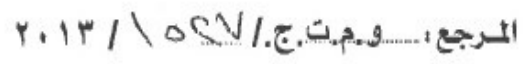

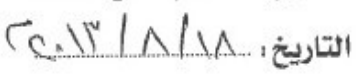

السيد الفاضل / د. وكيل الوزارة

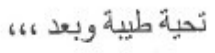

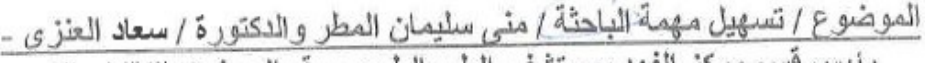

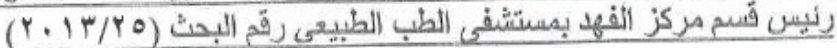

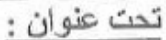

Evaluating Parental Interventions to Support the Management of Children with Cerebral Palsy

$$
\begin{aligned}
& \text { برجي التفضل بالإحاطة بأن اللجنة الدائمة لتنسيق البحوث الطبية و الصدية }
\end{aligned}
$$

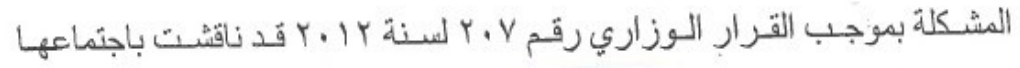

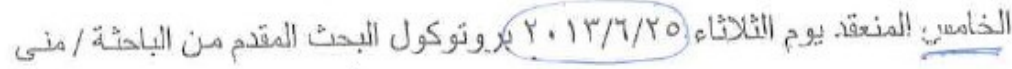

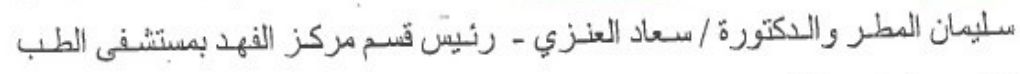

$$
\begin{aligned}
& \text { الطبيعي نحت عنوان : } \\
& \text { " تقييم التخخل العلاجي لأولياء الأمور لمسائدة علاج حالات الثطلل الدماغي " }
\end{aligned}
$$

\section{Evaluating Parental Interventions to Support the Management of}

\section{Children with Cerebral Palsy}

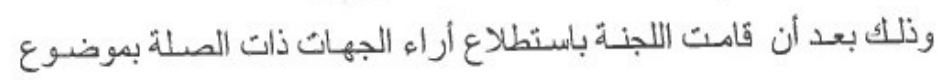

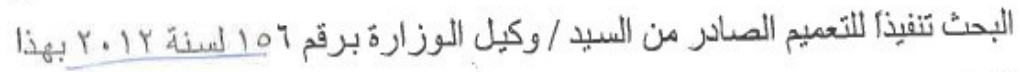

الشـان حيثت وردت المو افقة علبه من كل من السبد / مدير إدارة خدمات العـلاج

الطبيعسي بالكتــاب رقــمو.م.ت.ج.

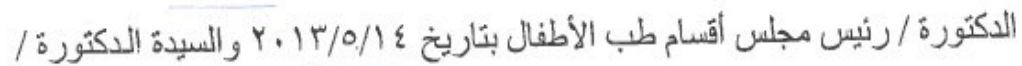

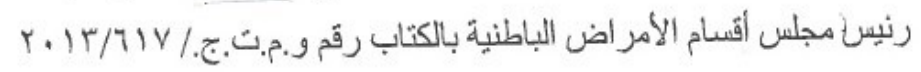

P.O. Box:(5) 13001 Safat, Kuwait

Tel : 24867159 - 24866724 Fax: 24877957 - 24866739

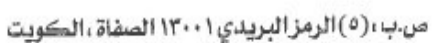

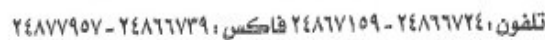


oubl delinisson

Skate of Kuwait

Ministry Of Health

Asst. Condorgeterciary for Planning \& Quality

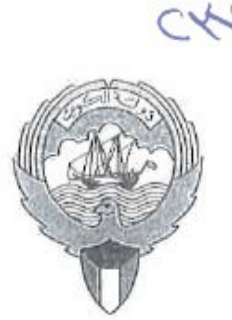

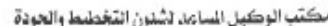

رقم الصاسر ،

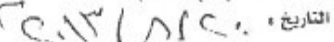
دولة|الكويت

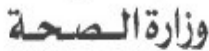

وكيل الوزارة المساعل الشئون التخطيطوالهوالجهدة

\section{Reference :}

Date :

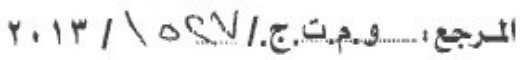

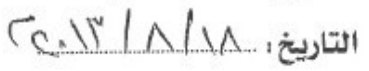

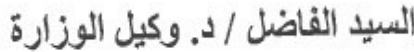

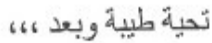

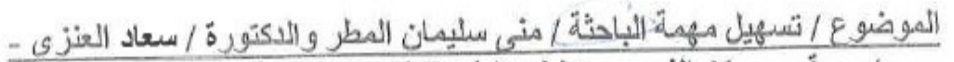

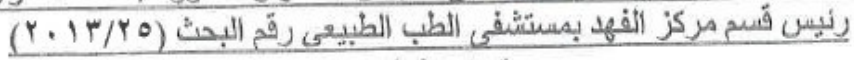
تصنت عنوان : الأن

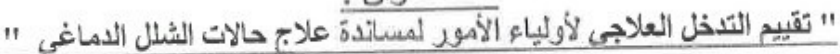

Evaluating Parental Interventions to Support the Management of Children with Cerebral Palsy

يرجي التفضل بالإحاطة بان اللجنة الدائمة لتنسيق البحوث الطبية و الصدية

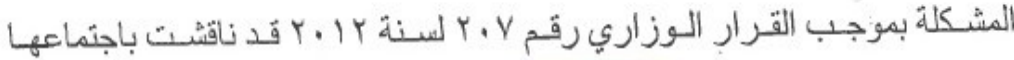

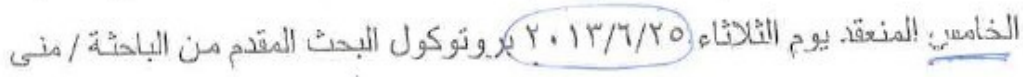

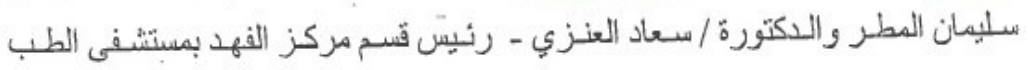

الطبيعي تصن عنوان :

" تقيييم التنخل العلاجي لأولياء الأمور لمسائدة علاج حالات الشطل الدماغي "

Evaluating Parental Interventions to Support the Management of Children with Cerebral Palsy

وذلك بعد أن قامت اللجنـة باسنطلاع أراء الجهاث ذات الصـلة بموضـوع

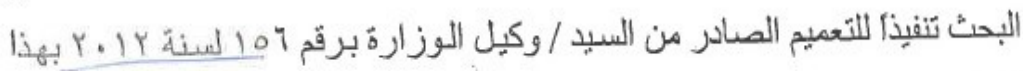

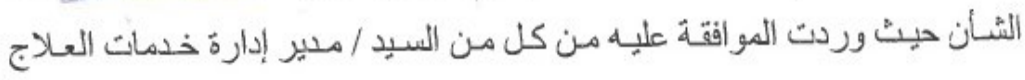

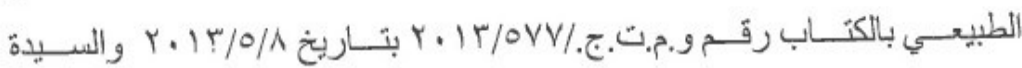

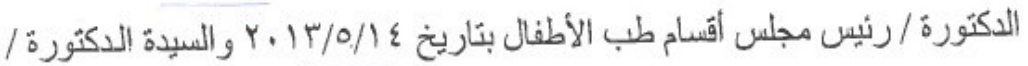

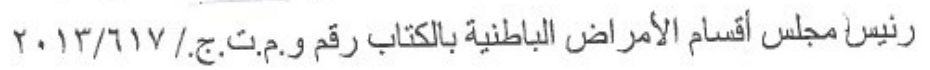

P.0. Box:(5) 13001 Safat, Kuwait

Tel : 24867159 - 24866724 Fax: 24877957 - 24866739

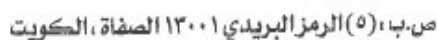

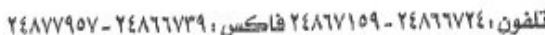




\section{APPENDIX 5}

\section{Ethical Approval from Swansea University REC}

From: Edwards, Steve

Sent: Tuesday, October 06, 2015 12:43 PM

To: ALMATER M.

Cc: Terry K.; NOBLE J.G.

Subject: REC application

$6 / 10 / 15$

Dear Mona,

Your application was approved at yesterday's meeting subject to your dealing with the following queries, each of which can be dealt with by chair's action:

1. Re section 1 and the two-day course for parents, can you describe in a sentence for me what these treatment techniques involve? 
invasive?

3. Re section 17 , can you say where the data collected in Kuwait will be stored and confirm it is secure?

4. Re the participant information sheet (PIS), this is too long and needs to be shortened.

5. Also (re the PIS again) can you make clear that there are three arms to the study and that participants will not be able to choose which one they are entered into. Can you also describe each arm briefly and clearly in the PIS?

6. Re questionnaire. Can you go through the questionnaire as the committee thought that some questions arenot applicable to all research groups.

7. Re the group who do not receive the research interventions, just the standard care, can you offer them a lecture/presentation at the end of the research outlining your results? This could be a helpful 'de-briefing' session for all involved in the study.

8. Re section 6, recruitment, can you take care to ensure that no participants will feel 'coerced' in to taking part in the study by stressing that there is no need to if they don't want to, and they can change their minds if they want to.

Can you respond to these points by email and I will then advise how to proceed?

Many thanks

Best wishes

Steve 
(Prof/Yr Athro) S.D.Edwards

Adeilad Vivian| Vivian Building

Prifysgol Abertawe | Swansea

University Parc Singleton

Singelton Park Abertawe $\backslash$ Swansea

SA2 8PP

Ffôn | Phone

\section{Ebost | email}

Mae'r Brifysgol yn croesawu gohebiaeth yn Gymraeg ac yn Saesneg | The University welcomes correspondence in Welsh and English.

From: ALMATER M.

Sent: 04 November 2015 11:23

To: Edwards, Steve

Subject: RE: REC application

Dear Mr Edwards

Here are my changes with respect to your comments:

1. Re section 1 and the two-day course for parents, can you describe in a sentence for me what these treatment techniques involve?

The techniques will include handling at home such as feeding dressing and bathing, in addition to preventing incorrect carrying, sitting positions and aggressive playing, and finally stretching and functional activities that are prescribed individually depending on each child's case. 
2. Re section 8 , the first paragraph says the care is 'non-physical', is this accurate or do you mean simply noninvasive?

Non physical and non invasive which means that the only treatment the child will be receiving will be from their own therapist. The researcher will not be providing any additional treatment what so ever. The home visits if applicable are simply to supervise the home program designed by the therapist and applied by the caregivers. The visits are only to ensure that the caregivers are applying the home program.

3. Re section 17 , can you say where the data collected in Kuwait will be stored and confirm it is secure?

The data collected in Kuwait will be stored in locked drawers and password protected computers inside the borders of Kuwait University and can only be accessed by the researcher and supervisors.

4. Re the participant information sheet (PIS), this is too long and needs to be shortened.

Have tried my best to shorten it, but unfortunately all the information available now should be there in order to meet up with the points in the consent form that the participants need to sign and agree to. 
5. Also (re the PIS again) can you make clear that there are three arms to the study and that participants will not be able to choose which one they are entered into. Can you also describe each arm briefly and clearly in the PIS?

I have highlighted the points required. (Attached)

6. Re questionnaire. Can you go through the questionnaire as the committee thought that some questions arenot applicable to all research groups.

Questionnaire was revised and improved. (Attached)

7. Re the group who do not receive the research interventions, just the standard care, can you offer them a lecture/presentation at the end of the research outlining your results? This could be a helpful 'de-briefing' session for all involved in the study.

A lecture outlining the results will be provided to the group of participants receiving the standard care. 
8. Re section 6 , recruitment, can you take care to ensure that no participants will feel 'coerced' in to taking part in the study by stressing that there is no need to if they don't want to, and they can change their minds if they want to.

Participants will be given the complete freedom of choice to take part in the study.

From: Edwards, Steve

Sent: Wednesday, November 04, 2015 11:51 AM

To: ALMATER M.

Cc: Terry K.; NOBLE J.G.

Subject: ATB/RE: REC application

\section{$4 / 11 / 15$}

Dear Mona,

Thank you for sending me the revised documentation and the application is now formally approved, Good luck with your research

(Prof/Yr Athro) S.D.Edwards

Adeilad Vivian| Vivian Building

Prifysgol Abertawe | Swansea

University Parc Singleton

Singelton Park Abertawe $\backslash$ Swansea

SA2 8PP

SA2 8PPFfôn | Phone

\section{Ebost | email}

Mae'r Brifysgol yn croesawu gohebiaeth yn Gymraeg ac yn Saesneg | The University welcomes correspondence in Welsh and English. 


\section{APPENDIX 6}

\section{Audit Result Sheet}

Q1) How was the child referred to the centre

1) Referrals from GPs

2) Referrals from paediatricians

3) Referrals from nurses

4) Cases that walk into physiotherapy centre

Q2) Number of previous regular physiotherapy treatment courses

Q3) Number of previous intensive physiotherapy treatment blocks

Q4) Number of cancellation of referrals

Q5) Number of cancelled appointments or Did Not Attend (DNA's)

Q6) Waiting times for treatment courses and intensive treatment blocks (Time from referral to first assessment)
1) .......Immediately to 2 weeks
2) ......2 weeks to 1 month
3) ......1 month to 2 months
4) .......More than 2 months

Q7) Time between blocks of treatment
1) .......Continuous treatment.
2) $\ldots . . .2$ weeks to one month
3) .....1 month to 2 months
4) .......More than 2 months 
Q8) Type of cerebral palsy:
1) ...... Spastic Diplegia
2) ...... Spastic Quadriplegia
3) ...... Spastic Hemiplegia
4) ...... Dystonic Quadriplegia
5) ...... Hypotonia
6) $\ldots . .$. Athetosis
7) ...... Ataxia
8) ...... Other

Q9) Level of cerebral palsy according to the GMFCS ( level I-V).
1) $\ldots . .$. level I
2) ...... level II
3) $\ldots . .$. level III
4) $\ldots . .$. level IV
5) ...... level $\mathrm{V}$

Q10) Was the session delivered once a week?
1) Yes
2) $\mathrm{No}$

Q11) Was the session delivered twice a week?
1) Yes

2) No

Q12) Was the session delivered 3 times a week?

1) Yes

2) $\mathrm{No}$

Q13) Was the session delivered daily?
1) Yes
2) $\mathrm{No}$

Q14) Was the session duration less than 1 hour
1) $\mathrm{Yes}$
2) $\mathrm{No}$

Q15) Was the session duration 1 hour

1) $\mathrm{Yes}$

2) $\mathrm{No}$ 
Q16) Was the session Duration more than 1 hour

1) Yes

2) $\mathrm{No}$

Q17) Did the patient file have an assessment sheet?

1) Yes

2) No

Q18) Did the patient file have an assessment tool

1) Yes

2) $\mathrm{No}$

Q19) Did the patient file have any outcome measures?
1) Yes
2) $\mathrm{No}$

Q20) Were Goals recorded using the SMART principle?
1) $\mathrm{Yes}$
2) No

Q21) Were goals recorded using the GAS principle?

1) Yes

2) $\mathrm{No}$

Q22) Were the set goals achieved?
1) Yes
2) $\mathrm{No}$

Q23) Was the home programme documented?
1) $\mathrm{Yes}$
2) No

Q24) Was there documentation of level of support provided from therapist to parent
1) Yes
2) $\mathrm{No}$

Q25) Was there documentation of details of the treatment techniques taught
1) Yes

2) $\mathrm{No}$

Q26) Was there documentation of detail of the programme's effect

1) Yes

2) No 

1) res

2) No

Q28) Was there documentation of the amount of follow up of the application of home programme

1) Yes

2) No

Q29) Was there documentation of amount of update to the home programme given to the parents throughout the treatment course.

1) Yes

2) No

Q30) The documentation for progression notes
1) $\ldots \ldots$ No progress sheet
2) $\ldots \ldots$ One progress sheet
3)..... More than one progress sheet 


\section{APPENDIX 7}

\section{Information Sheet Group}

\section{- Title:}

Evaluating current clinical practice and the involvement of parents to improve the clinical outcomes in the treatment of children with cerebral palsy using the Bobath approach.

- Purpose of study:

The study was done to assess the effectiveness of the Bobath approach after taking brake periods between the treatment courses. It also assesses the effect of the home programme delivered by the caregiver in supporting the effectiveness of the treatment delivered.

- Why your child have been chosen:

Your child represents the population required for this study of being a cerebral palsy child.

- Who is organising this study:

This study is organised by Mona AlMater a PhD student in Swansea University and is supervised Dr Gareth Noble.

- What will happen to you if you decide to take part?

You have a chance to receive a 2 day educational course of the home programme that will include both a theory and practical part. 
- What will happen to your child if you decide to take part?

If you decide for your child to take place in this study, you will sign a consent form assuring your approval of:

1. Once you decide to participate in this study a letter of information regarding your participation will be sent to your general practitioner (GP).

2. Assessing your child's CP level using a measurement scale called the GMFCS which includes observing the child's movement ability and the amount of independence in movements to confirm his CP level.

3. Providing a measurement session done by another qualified physiotherapist to assess the initial state of activity level before starting the treatment course using the GMFM88 which includes allowing the child to perform specific functional tasks and observing the level of independence in performing these tasks.

4. Providing a full assessment by a therapist of your child's motor development in lying, sitting, standing and walking using the GMFM88.

5. Providing your child a treatment course of 6 weeks of a frequency of 2-3 sessions per week of Bobath inspired treatment techniques that include weight bearing and weight shifting functional activities and play therapy.

6. You providing the home programme learnt from the educational course and pointed out by the researcher throughout the course.

7. Providing a second measurement session by the supporting therapist to assess the amount of progress gained after the treatment course.

8. Stopping the treatment for an off period of 6 weeks which will require you to continue with providing your home treatment

9. You might receive a weekly visit from the researcher for supervision and encouragement. 
10. Providing a third measurement session by the supporting physiotherapist to assess the amount of change acquired after the off period.

- Measurement tools:

1. The GMFCS is a measurement scale that allows the researcher to assess the CP level of the child participating in the study through assessing the child's ability of independent movements.

2. The GMFM is a measurement tool that assesses the child's ability to perform movements and activities to indicate the child's progress in response to treatment.

- Disadvantages of participating:

1. Your child will have to be handled by a new physiotherapist who is unfamiliar to them which might be uncomfortable for your child.

2. Your child will be required to be committed to all the treatment and measurement sessions.

3. Your child will be excluded from the study if he/she misses 3 sessions of treatment.

- Possible risks:

1. During the treatment the patient may have to be put in uncomfortable positions that they are not familiar with such as the crawling position.

2. The patient may experience some pain during some treatment exercises.

3. The patient might suffer some fatigue from the treatment sessions.

The patient might face minimal risk of falling on a mat during gait training or during other exercises. 
- Benefits of participating:

1. The caregiver might benefit from a 2 day education course that will improve the level of understanding the disability you child has and learn the importance and the effects of applying the home treatment.

2. The patient might benefit from the home visit applied by the researcher.

3. The patient will participate in supporting research that would improve the level of CP treatment.

- Will you be paid to participate:

You will not be paid for participating, and neither the researcher will be paid for participating.

- Will your identity be exposed:

All the participant children and parents will be anonymous except for the researchers and supervisor.

- Will there be restrictions prior to participating:

1. The child will be required to be well fed one hour before the session to prevent any discomfort or heaviness or hunger during the session.

2. The patient will be required to come to the session after a good night sleep or after a good nap to prevent the child being sleepy during the session.

3. The patient will be required to be in a good health with no fever or any infections to prevent any complications and to promote best benefits and results from the session. 
- What will happen to the data collected:

The data will be analysed and results will be calculated and possibly published.

- Contacts:

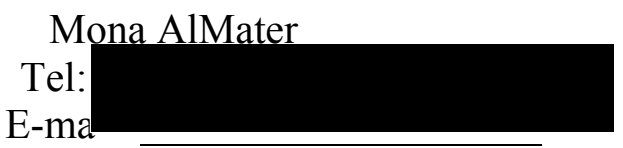




\section{APPENDIX 8}

\section{Consent Form}

\section{Title of the study:}

Evaluating current clinical practice and the involvement of parents to improve the clinical outcomes in the treatment of children with cerebral palsy using the Bobath approach.

Tick as appropriate

- We confirm that we have read and understood the informative sheet given to us and we were welcomed to ask any questions and we were answered clearly.

- We understand that the participation of our child is completely optional and we have participated of our own will, and we are free to withdraw at any point without affecting our treatment.

- We therefore confirm and approve of all the procedures and steps required from our child to complete this study.

- We therefore give permission to the researcher to take pictures and or videos for teaching purposes as long as our identity remains anonymous. 
- We understand that all our personal information will remain confidential and will be destroyed at the end of this study.

I agree to take part in this study:

Name of participant Signature. Date.

Witness Signature Date

The procedures and tasks were fully defined and explained to the participants in whom he/she have agreed to participate and signed the consent.

Researcher. Signature

Date 


\section{APPENDIX 9}

\section{Service Evaluation Questionnaire}

\begin{tabular}{|l|l|}
\hline Patient code: & \\
\hline patient age: & \\
\hline $\begin{array}{l}\text { Patient } \\
\text { diagnosis: }\end{array}$ & \\
\hline
\end{tabular}

Ques 1) How long has your child been receiving physiotherapy sessions?
1) 1-3 months
2) 3-6 months
3) 6-12 months
4) More than 12 months

What types of physiotherapy sessions has your child been receiving? How would you rate it? If fair, bad or very bad could you specify why?

Quest 2) Intensive treatment
1) Very good
2) Good
3) Fair
4) $\mathrm{Bad}$
5) Very bad

Quest 3) Normal sessions
1) Very good
2) Good
3) Fair
4) $\mathrm{Bad}$
5) Vary bad 
Quest 4) Hydrotherapy

1) Very good
2) Good
3) Fair
........................

5) Very bad

Quest 5) Gait treatment
1) Very good
2) Good
3) Fair
4) $\mathrm{Bad}$

5) Very bad

Quest 6) Casting
1) Very good
2) Good
3) Fair
4) $\mathrm{Bad}$
5) Very bad

Quest 7) Were there any difficulties for you in coping with the frequency of bringing your child to the sessions?
1) Yes
2) $\mathrm{No}$
3) A little
4) I don't know 
Quest 8) Were there any difference observed in your child's condition?

1) My child has improved

2) My child showed no change

3) My child has deteriorated

4) I don't know

Quest 9) Have you received any education throughout your treatment courses of your child?

1) Yes

2) No

3) I don't know

Quest 10) If Yes can you rate it?

1) Very good

2) Good

3) Fair ..

4) $\mathrm{Bad}$ .$\cdot$

5) Very bad

Quest 11) Would you suggest anything to improve the education that you have received if you have?

Quest 12) Would you suggest anything to improve the services you have been receiving? 


\section{APPENDIX 10}

\section{Home Program evaluation}

Q1) Were you given a home programme to apply at home after the sessions have taken place?
1) Yes
2) No

Q2) If no, will you accept to learn it and apply it if it was offered to you?
1) Yes
2) No

If No, can you please specify why.....

Q3) If you were given a home programme, was the programme given to you on a paper?
1) Yes
2) No

If No, can you please specify how it was given.....

Q4) Were the exercises of the home programme clearly explained to you by the researcher?
1) Yes
2) No

Q5) Were the exercises demonstrated to you?
1) Yes
2) No

Q6) Were you given the proper time to understand and practice the exercises with the therapist after receiving the exercises?
1) Yes
2) No

Q7) Was the researcher open to any further inquiries and questions about the home programme?
1) Yes
2) No

Q8) Did the therapist explain the purpose and importance of understanding and applying the home programme in the proper frequency and intensity?
1) Yes
2) No

Q9) Were the exercises easy to understand and apply?
1) Yes
2) No

If No, can you please specify why

Q10)Were you committed to the frequency of application advised by the therapist?
1) Yes
2) No

If No, can you please specify why. 
Q11) Did you find any difficulties in applying the home programme at home?
1) Yes
2) $\mathrm{No}$

If No, can you please specify why

Q12) Was the home programme stressful?
1) Yes
2) $\mathrm{No}$

Q13) Did you see any changes or progress from the home programme on your child's condition? 1) Yes 2) No

Q14) If Yes, were the changes beneficial?
1) Yes
2) No 


\section{APPENDIX 11}

\section{Education Lecture}

YOUR CHILD AND CEREBRAL PALSY

\section{OBJECTIVES:}

- Provide caregivers with the basic information of cerebral palsy

- Correcting myths and incorrect information regarding 
DAMAGE

1) Decreased embryotic development

2) Premature delivery

3) Low birth weight

These circumstances result in decreased systematic functional level such as axyren delivery cansine
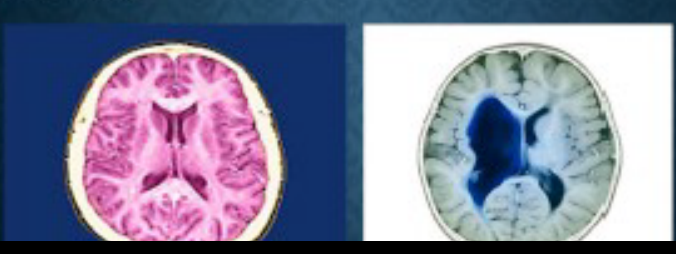


\section{IDENTIFYING THE CASE:}

- The main symptom that may indicate serebral palsy is the delay in milestones such as rolling creeping and sitting. Other important symptoms are the increase in tone in upper and lower limbs in addition

\section{EARLY INTERVENTION}

- Some cases are known as high risk babies. In such cases children have a history of risks during pregnancy or labor. Therefore a child is placed under observation in order to identify any abnormalities in its early stages.

- Early intervention for cerebral palsy cases prevents the child 


\section{INFORMATION EXCHANGE:}

- Information exchange betwreen family and medieal team is considered the ideal way of understanding the child's

condition in order to nrovide care suitable for the child's
UNDERSTANDING MOVEMENT AND

DEVELOPMENT:

- Movement is produced through a group of muscular enntractions not inet a sincle

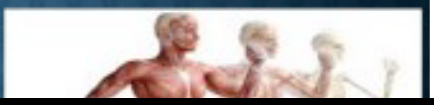


2) Bouncing on the feet which also increases the tone in back thigh muscles, inner thigh muscles and calf muscles leading to tiptoeing. 


\section{APPENDIX 12}

\section{Parents Satisfaction Questionnaire}

Q1) Were the procedures of the trial clearly explained to you by the researcher after reading the information sheet?
1) Yes
2) No

If no please specify how

Q2) Were you given the freedom and the proper time in order to decide whether or not to take place in the trial?
1) Yes
2) No
If no please specify how

Q3) Was the researcher open to any further inquiries and questions about the trial?
1) Yes
2) No

If no please specify how 
Q4) Were you happy about joining the study?
1) Yes
2) No

If no please specify why.

Q5) Were you given the freedom to withdraw at any time from the trial without any questions asked?
1) Yes
2) No

If no please specify how.

Q6) Did you benefit from the educational course given to you in the beginning of the trial?
1) Yes
2) A little
3) $\mathrm{No}$

If not yes please specify why. 
Q7) Did your child adapt quickly to the new assessing therapist
1) Yes
2) No

If no please specify why.

Q8) Did you have any doubts regarding the wellbeing of your child before or during the trial?
1) Yes
2) A little
3) No

If not no please specify why.

Q9) Was the frequency of the treatment session convenient to you?
1) Yes
2) A little
3) No

If not yes please specify why

Q10) Were there any difficulties for you in coping with the frequency of bringing your child to the sessions? 
2) A little

3) $\mathrm{No}$

If yes please specify how

Q11) Was the frequency of the home visits from the therapist convenient to you
1) Yes
2) A little
3) $\mathrm{No}$

If not yes please specify why

Q12) If You Haven't received any home visits, have you applied the home programme learnt during your treatment sessions?
1) Yes
2) $\mathrm{No}$

If not can you specify why......

Q13) If yes, how many times did you apply the home programme at home?
1) Daily
2) 3-5 times a week
3) Less than $3 X$ a week 
Q14) Was there any difference observed in your child's progress?

1) Yes

2) No

Q15) Were the procedures explained before taking part in the trial the same as what you have experienced in the trial?

1) Yes

2) No

If no please specify how

Q16) At the end of the trial are you happy that you joined the trial Yes

A little

No

If not please specify why.

Q17) Why did you decide to join the trial?

Q18) Any further comments or suggestions. 


\section{APPENDIX 13}

\section{Physiotherapist Questionnaire}

\section{Evaluating current clinical practice and the involvement of parents to improve the clinical outcomes in the treatment of children with cerebral palsy using the Bobath approach}

\section{Welcome}

Thank you for taking an interest in this study and finding time to participate in our online questionnaire.

The aim of this project is to evaluate the physiotherapy services and effect of treatment of children with cerebral palsy when supported by the caregivers via home programme.

There will be several basic background questions related to demographics, followed by specific questions regarding the use of Bobath in the management of children presenting with cerebral palsy within your clinical practice. Most questions are multiple choice, occasionally with space to provide additional details. The survey should take 20-30 minutes to complete.

If there are questions you do not wish to answer please leave them blank. Alternatively, if a question does not apply, please answer "N/A" where possible. If you wish to withdraw from the study during the questionnaire simply close the browser, no data is sent unless the questionnaire is completed. However, please note that due to the anonymous nature of the questionnaire, it will not be possible to identify your data for withdrawal after submission.

Data in this survey is being collected by Mona Al-Mater at Swansea University Medical School under the supervision of Dr Melanie Healy and Dr Gareth Noble, with approval from Swansea University College of Human and Health Sciences Research Ethics Committee.

Analysis of the data will be used to form a PhD thesis at the end of the study, which will then be published in scientific journals. Information provided will remain anonymous in all publication.

If you have any questions relating to the questionnaire or the study, please contact Mona Al-Mater, Dr Melanie Healy or Dr Gareth Noble using the following methods:

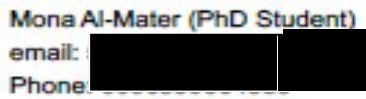

Dr Gareth Noble

email:

phone 
You are invited to participate in this online questionnaire which aims to assess the general opinion of the physiotherapists regarding the provision of cerebral palsy services including the Bobath approach in Kuwait.

This questionnaire is part of an on-going project that aims to explore the current clinical practice with the involvement of parents to improve clinical outcomes in the treatment of children with cerebral palsy using the Bobath approach. This study is being conducted as part of a thesis project of the PhD course I am registered for with Swansea University.

What is the purpose of the study?

As physiotherapy, with all its various interventions, becomes an important part of the treatment of cerebral palsy; it becomes essential that such interventions have an evidence base to support its continued clinical application for the management of developmental related disability. Many studies have assessed the validity of Bobath therapy as a major treatment approach. However, although the literature has been assessing the most suitable approach for cerebral palsy, little evidence exists today that supports the clinical application of Bobath for CP or to determine which aspects of it has the most influence upon clinical outcome. The purpose of this study is to assess the opinions of physiotherapists regarding the parameters of the services being delivered to the children with $\mathrm{CP}$ especially the Bobath approach in addition to assessing the views of physiotherapists regarding the effectiveness and the continuous effect of the Bobath approach with its different principles in the management of the symptoms of cerebral palsy.

Why have I been invited to participate?

If you are a physiotherapist providing physiotherapy services for children with cerebral palsy, you are invited to participate in this online questionnaire.

Do I have to take part?

No. Taking part in the study is entirely voluntary as it is entirely up to you to decide whether, or not, you wish to take part. If you do decide to take part, you will still be completely free to withdraw from the study, at any time, without having to give a reason.

What will happen to me if I take part?

If you decide to take part in this questionnaire study, you will firstly be asked for your consent to use any information you provide by completing the questionnaire for the purposes of this research project. You will also be asked to confirm you are over 18 year of age and to identify if you are a qualified physiotherapist.

You will then be allowed to proceed to the questionnaire, where you will be asked a series of questions that provide background information about you (but you will not be asked to identify yourself, i.e. date of birth, address etc) and your qualifications as a physiotherapist in addition to the years of experience that you have gained in the field of paediatric physiotherapy and years of experience in your current department of duty. The questions will then begin regarding the Cerebral Palsy services.

The questionnaire will take about 20-30mins at most to complete. Your responses will be stored on a password protected Swansea University based computer and will only be accessed by the researchers involved with this project.

What will happen if I do not want to carry on with the questionnaire once I have started it?

You will be able to leave the online questionnaire at anytime and any responses you have made will be deleted and removed. It is your right to withdraw from the study at any time.

What are the possible benefits of taking part?

The information that we will obtain from this study will help us to evaluate the physiotherapy services for children with cerebral palsy in addition the views of the physiotherapist working in this field. 


\section{Evaluating current clinical practice and the involvement of parents to improve the clinical}

outcomes in the treatment of children with cerebral palsy using the Bobath approach

\section{Participant Information Continued...}

What happens when the study finishes?

When the study finishes, the researchers will be writing a summary report of the results. If you wish to receive a copy of the summary, you will need to email one of the project supervisors (their contact details are at the end of this introduction). We will also be writing a paper for publication. No participants in the study will be personally identified in summary reports or publications. The information you provide via this online questionnaire will be stored on a secure and password protected computer for a period of 3 years, after which time all data collected will be deleted.

Will my taking part in the study be kept confidential?

You will not be asked any questions that would provide answers that could identify you. All information you provide will be handled and kept in strict confidence, and all information from this study will be anonymous.

What happens if there is a problem?

Any complaints or problems that you may have regarding the study will be addressed. Should any issue arise during the study that you are unhappy with, please do not hesitate to contact one of the project supervisors, who will do their very best to answer your questions.

Who is organizing and funding the research?

The research is organized by Swansea University Medical School as part requirement for the submission of a thesis for the award of PhD in Paediatric Physiotherapy.

Who has reviewed the study?

The study has been reviewed by the College of Human and Health Sciences and College of Medicine Research Ethics Committee, to protect your safety, rights, wellbeing, and dignity.

If, after reading this, you are still unsure about whether you would like to take part in this research study, you may find it useful to talk with any member of the research project team (contact details below).

If you have any further issues regarding this questionnaire please contact any of the contacts in charge of the study, the contact details are listed below.

In case you have any questions or comments on this questionnaire, please contact any of the contacts listed below.

\section{Mona AlMater (PhD Student)}

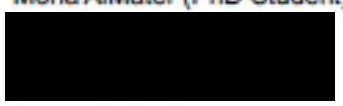

Dr Melanie Healy (Supervisor)

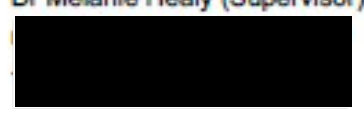

Dr Gareth Noble (Supervisor)

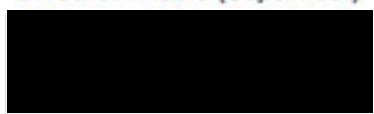


Evaluating current clinical practice and the involvement of parents to improve the clinical outcomes in the treatment of children with cerebral palsy using the Bobath approach

\section{Participant Consent}

Project Title: Evaluating current clinical practice and the involvement of parents to improve the clinical outcomes in the treatment of children with cerebral palsy using the Bobath approach

Name(s) of Researcher(s):

Mona Al-Mater (Physiotherapist/PhD Student)

Dr Melanie Healy (Research Project Supervisor)

Dr Gareth Noble (Research Project Supervisor)

Aims of the Research:

The aim of this questionnaire is to assess the general opinion of physiotherapists regarding the provision of cerebral palsy services including the Bobath approach.

Contact Details of Researcher:

Phone:

e-mail:

\section{* 1. Participant Consent}

I confirm that I have read and understood the information sheet provided for the above study

I understand that my participation is voluntary and that I am free to withdraw at any time, without giving a reason

I agree to take part in the above study

I I confirm that I am over 18 years of age

I I confirm that I am a qualified physiotherapist 
Evaluating current clinical practice and the involvement of parents to improve the clinical outcomes in the treatment of children with cerebral palsy using the Bobath approach

General Participant Information

This section of the questionnaire will ask you general questions about you as a physiotherapist.

* 2. What is your Gender?
Male
Female

* 3 . What is your age range?
$20-25$
$26-30$
$31-35$
$36-40$
$40-45$
$46-50$
( $51-55$
( $56-60$
Above 60

* 4. What is your highest qualification?

Diploma in Physiotherapy

Bachelor's degree in physiotherapy (BSc)

Masters in physiotherapy (MSc)

Other (please specify)

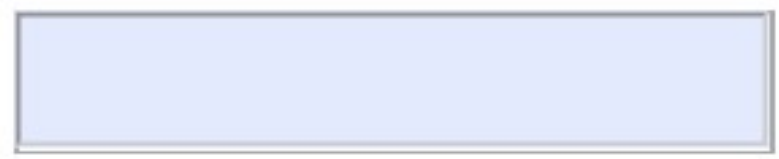


* 5. How many years of experience do you have as a paediatric physiotherpist?

$1-5$ years

6-10 years

11-15 years

More than 15 years

* 6. How many years of experience do you have working in your current department?

$1-5$ years

(D-10 years

11-15 years

More than 15 years

* 7. What is your level of training regarding Bobath therapy?

Bobath trained

Receiving training

Supervised by a Bobath trained therapist (provide Bobath influenced therapy)

Not trained 
outcomes in the treatment of children with cerebral palsy using the Bobath approach

About Cerebral Palsy Services

* 8. How many cases of children presenting with Cerebral Palsy do you see on average per week?

$1-3$ cases

$4-6$ cases

7-9 cases

$10-12$ cases

More than 12 cases

* 9. What outcome measures do you use for assessing the patients' physical function?

Range of Motion

Modified Ashworth scale of tone

$\square$ General Motor Functional measurement (GMFM)

$\square$ General Motor functional Classification System (GMFCS)

$\square$ Quality of Upper Extremity Skills Test (QUEST)

Paediatric Functional Independence Measure (Wee FIM)

Beery- Buktenica Developmental Test of Visual-Motor Integration (Beery VMI)

Other (please specify)

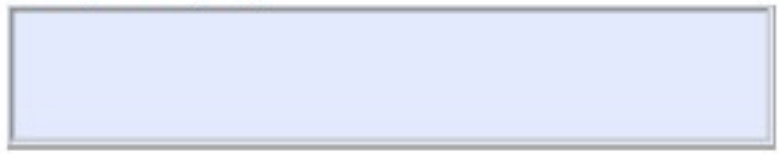




\section{Range of Motion}

Modified Ashworth scale

of tone

General Motor

Functional measurement

(GMFM)

\section{General Motor functional}

Classification System

(GMFCS)

\section{Quality of Upper}

Extremity Skills Test

(QUEST)

\section{Paediatric Functional}

Independence Measure

(Wee FIM)

\section{Beery-Buktenica}

Developmental Test of

Visual-Motor Integration

(Beery VMI)

* 11. Please indicate which of the following outcome measures do you believe has the most clinical value to assessing the outcome of treatments in children with cerebral palsy?

Range of Motion
Modified Ashworth scale
of tone
General Motor
Functional measurement
(GMFM)
General Motor functional
Classification System
(GMFCS)
Quality of Upper
Extremity Skills Test
(QUEST)
Paediatric Functional
Independence Measure
(Wee FIM)
Beery- Buktenica
Developmental Test of
Visual-Motor Integration
(Beery VMI)




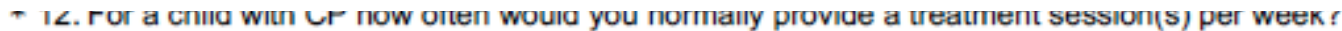

1 session per week

2 sessions per week

3 sessions per week

Daily

Other (please specify) 


\section{About the Principles of Bobath Therapy}

* 13. Do you think Bobath is a widely used approach around the world?
Yes
No
I don't know

* 14. Do you think that all CP children should receive Bobath therapy?
Yes
No
I don't know

Can please justify your answer

* 15. Do you think that Bobath therapy is more effective than other treatment approaches for children with $\mathrm{CP}$ ?
Yes
No
I dont know

Can you please justify your answer 
cerumed training?

Yes

No

I don't know

Can you please justify your answer

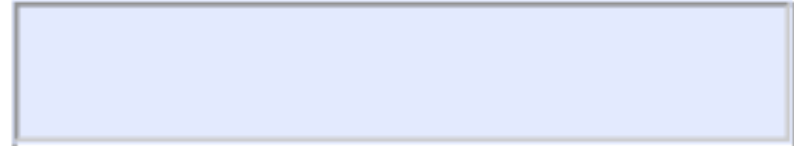

* 17. Do you believe that Bobath therapy is being delivered in the right parameters of intensity for patients with $\mathrm{CP}$ ?
Yes
No
I don't know

Can you please justify your answer

* 18. Do you think that the Bobath approach is effective in inhibiting static and motor tone?

Yes

(To

I dont know

Can you please justify your answer 
posture or cnilaren witn Lr?

( Yes

( No

I don't know

Can you please justify your answer

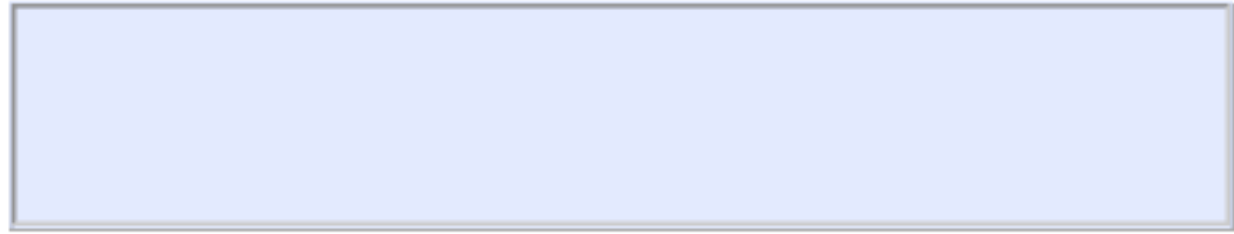

* 20. Do you think that the weight bearing principle of the Bobath approach is effective in decreasing tone and strengthening muscles of children with $\mathrm{CP}$ ?

Yes

No

I don't know

Can you please justify your answer

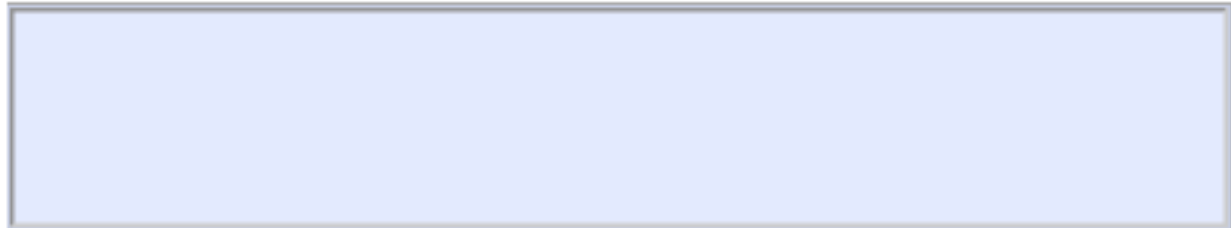

* 21. Do you think that the principle of inhibiting primitive reflexes of the Bobath approach is effective with children with $\mathrm{CP}$ ?
Yes
( No
I don't know
Can you please justify your answer 
outcomes in the treatment of children with cerebral palsy using the Bobath approach

Involving Parents/Caregivers in Treatment of Children with CP

* 22. To what extent do you think the home programme supports components of the Bobath approach?

Major support

Moderate support

Minimal support

No support at all

* 23 . Are you currently providing your patients with a home programme to apply at home?

Yes

No

Occasionally

* 24. To which extent do you think that your patients' caregivers are committed to the home programme?

Very committed

Partially committed

Not at all committed

* 25 . If you are providing a home programme, in which way are you providing the programme?

Documented images

Practical demonstration

$\square$ Verbal explanation

* 26. Do you practically see the effect of the home programme on the prognosis of your patients?

Yes

To an extent

No 
* 27 . Should there be more focus on educating the caregivers regarding the home programme?

(?) Yes

To an extent

No

28. What suggestions do you have for improving the education of caregivers regarding the home programme? 
Evaluating current clinical practice and the involvement of parents to improve the clinical outcomes in the treatment of children with cerebral palsy using the Bobath approach

Any Other Comments

29. Other Comments 
Evaluating current clinical practice and the involvement of parents to improve the clinical outcomes in the treatment of children with cerebral palsy using the Bobath approach

Thank you

Thank you for completing this survey on the use of Bobath Therapy in the management of children with cerebral palsy.

If you have any comments or questions, please do not hesitate to contact us on the following email addresses:

PhD Student: Mona Al-Matel

Supervisor: Dr Gareth Noble

Supervisor: Dr Mel Healy (M 